University of Louisville

ThinkIR: The University of Louisville's Institutional Repository

Electronic Theses and Dissertations

$5-2017$

\title{
Electronic nose for analysis of volatile organic compounds in air and exhaled breath.
}

Zhenzhen Xie

University of Louisville

Follow this and additional works at: https://ir.library.louisville.edu/etd

Part of the Engineering Commons

\section{Recommended Citation}

Xie, Zhenzhen, "Electronic nose for analysis of volatile organic compounds in air and exhaled breath." (2017). Electronic Theses and Dissertations. Paper 2707.

https://doi.org/10.18297/etd/2707

This Doctoral Dissertation is brought to you for free and open access by ThinkIR: The University of Louisville's Institutional Repository. It has been accepted for inclusion in Electronic Theses and Dissertations by an authorized administrator of ThinkIR: The University of Louisville's Institutional Repository. This title appears here courtesy of the author, who has retained all other copyrights. For more information, please contact thinkir@louisville.edu. 
ELECTRONIC NOSE FOR ANALYSIS OF VOLATILE ORGANIC COMPOUNDS

IN AIR AND EXHALED BREATH

\author{
By \\ Zhenzhen Xie \\ M.S., University of Louisville, 2013 \\ B.S., Heilongjiang University, 2011
}

\begin{abstract}
A Dissertation
Submitted to the Faculty of the J. B. Speed School of Engineering University of Louisville in Partial Fulfillment of the Requirements

for the Degree of

Doctor of Philosophy

in Chemical Engineering

Department of Chemical Engineering

Louisville, KY
\end{abstract}

May 2017 

ELECTRONIC NOSE FOR ANALYSIS OF VOLATILE ORGANIC

COMPOUNDS IN AIR AND EXHALED BREATH

by

Zhenzhen Xie

B.S., Heilongjiang University, 2011

M.S., University of Louisville, 2013

A Dissertation Approved On

04/03/2017

by the Following Committee:

Dr. Xiao-An Fu, Dissertation Director

Dr. Michael Nantz

Dr. James C. Watters

Dr. Gerold A. Willing

Dr. Gamini U. Sumanasekera 


\section{DEDICATION}

\section{This Dissertation is dedicated to my lovely parents}

\section{Zhonghua Xie and Baiqin Feng}

Who have given me invaluable educational opportunities and make me who I am

And

Friends 


\section{ACKNOWLEDGMENTS}

First of all, I would like to express my deepest gratitude to my advisor, Prof. Xiaoan $\mathrm{Fu}$, for his guidance, support and encouragement through my research and writing. Dr. Fu introduced me to a new and exciting area of research and provided an exciting working environment with many opportunities to explore new ideas. It would not have been possible without his professional advice and guidance.

I would like to thank my committee members, Professor Michael Nantz, Professor James C. Watters, Professor Gerold A. Willing, and Professor Gamini U. Sumanasekera for offering their time and support to help me complete my research

project. Special thanks to Professor Nantz for his valuable advice of synthesizing different thiols throughout the project, which helps me a lot on the progress of my project.

Many thanks to Dr. Mandapati Raju, who helped me synthesize different groups of thiol and showed me the principle of chemistry behind the scene. I would also like to thank Dr. Yehya Senousy, Dr. Yizheng Chen, Dr Mingxiao Li, Jared Stephens, Qi Li and Mumiye Ogunwale for their assistance in the lab. They always have discussions with me whenever I confront any difficulties. They helped create a very positive work environment. 
I would like to thank Dr. Jacek Jasinsski for his help and training in material characterization. Also, many thanks to all the staff and technicians in the Department of Chemical Engineering at University of Louisville, who helped me in solving all the problems and I came to know about so many new things.

I would also like to thank Ms. Patricia Lumley who has guided me through all the procedures to get my degree.

I would like to thank my husband Jordan Bray who has always supported me in this progress. He comforts me whenever I feel overwhelmed. I'm very glad we have Simon (our dog) in our life. He is such a cutie and he brings us so much happiness. Thanks also to all my friends who have helped me in finishing my Ph.D. degree.

And last, but not least, my greatest thankfulness goes to my family for their love and support and kind co-operation and encouragement. My Mom is sick when I'm on the last year of finishing my Ph.D. degree. I'm really sorry that I can't be with you to fight the disease. I'm glad that we can talk on the phone or via facetime, so I know your situation all the time. I hope you'll recover soon. I love you, my lovely family. Without your understanding and support, this dissertation and research would not have been possible. 


\title{
ABSTRACT \\ ELECTRONIC NOSE FOR ANALYSIS OF VOLATILE ORGANIC COMPOUNDS IN AIR AND EXHALED BREATH
}

\author{
Zhenzhen Xie
}

$04 / 03 / 2017$

Exhaled breath is a complex mixture containing numerous volatile organic compounds (VOCs) at trace levels (ppb to ppt) including hydrocarbons, alcohols, ketones, aldehydes, esters and other non-volatile compounds. Different patterns of VOCs have been correlated with various diseases. The concentration levels of VOCs in exhaled breath depend on an individual subject's health status. Therefore, breath analysis has great potential for clinical diagnostics, monitoring therapeutic progress and drug metabolic products. Even though up to 3000 compounds may be detected in breath, the matrix of exhaled breath is less complex than that of blood or other body fluids. Breath analysis can be performed on people irrespective of age, gender, lifestyle, or other confounding factors. Breath gas concentration can be related to VOC concentrations in blood via mathematical modeling; for example, as in blood alcohol testing. Since exhaled breath samples are easy to collect and online instruments are commercially available, VOC analysis in exhaled breath appears to be a promising tool for noninvasive detection and monitoring of diseases. Breath analysis has been very successful in identifying cancer, diabetes and other diseases by 
using a chemiresistor sensor array to detect biomarkers.

The objective of this research project is to develop sensor arrays - or so-called electronic nose - for analysis of VOCs in air and exhaled breath. In this dissertation, we have investigated both commercial and synthesized thiol functionalized gold nanoparticles (AuNPs) as sensing materials for analysis of VOCs in air and exhaled breath. The advantages of these sensors include very high sensitivity, selectivity for detection of target analytes and operation at ambient temperature. The synthesis and material characterization of new thiols and AuNPs for increasing sensitivity and selectivity have been studied.

Selected commercial thiols and in-house synthesized new functional thiols have been used to modify AuNP-based sensors for detection of VOCs in air and exhaled breath. The interdigitated electrodes (IDE) used for the sensors were fabricated by microelectromechanical systems (MEMS) microfabrication technologies. The sensor arrays were characterized by measuring the resistance difference from vacuum and different spiked analyte concentrations in air and breath samples. Air samples and breath samples were collected using Tedlar bags, and analyzed using the thiol functionalized AuNP sensors. The analysis of air samples provides a reference for analysis of exhaled breath samples.

The sensors have demonstrated a low detection limit of $0.1 \mathrm{ppbv}$ of acetone and ethanol in dry air and exhaled breath. The concentrations of acetone in air and exhaled breath were determined by a silicon microreactor approach. The measurements of acetone by the microreactor approach were correlated with the sensor signals. The intellectual thrust of this research is the rational design of an electronic nose for analysis of VOCs in 
exhaled breath, which offers a new frontier in medical diagnostics because of its noninvasive and inexpensive characteristics. 


\section{TABLE OF CONTENTS}

ACKNOWLEDGMENTS ..................................................................................... iv

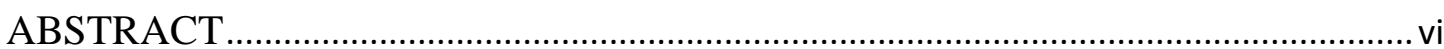

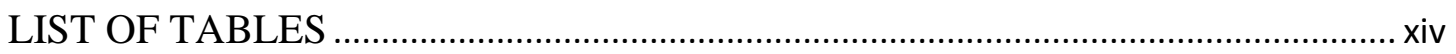

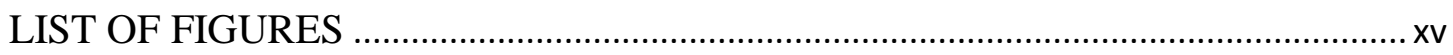

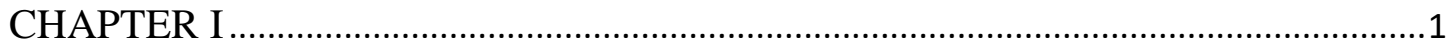

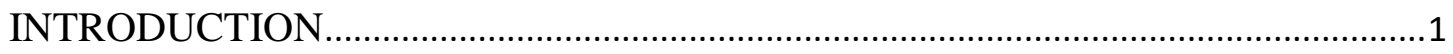

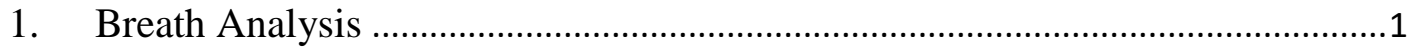

2. GC or MS Based Techniques for VOC Detection .........................................

2.1 Gas Chromatography-Mass Spectrometry (GC-MS) ................................6

2.2 Selected Ion Flow Tube Mass Spectrometry (SIFT-MS) ...........................

2.3 Proton Transfer Reaction-Mass Spectrometry (PTR-MS) ........................10

2.4 Ion Mobility Spectrometry (IMS) …..................................................12

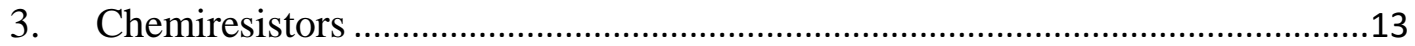

4. Electronic Nose for VOC detection ...........................................................

4.1 Metal Oxide Semiconductor Sensors ........................................................17

4.2 Nanomaterial Sensors ............................................................................. 19

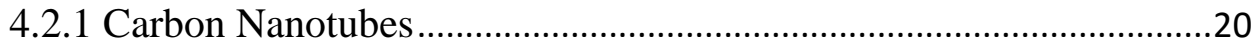

4.2.2 Metal Nanoparticles ..................................................................22 


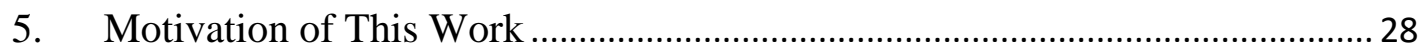

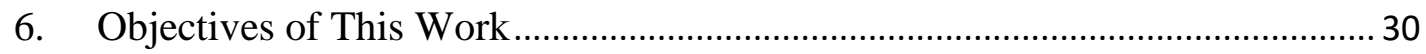

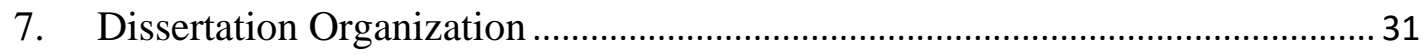

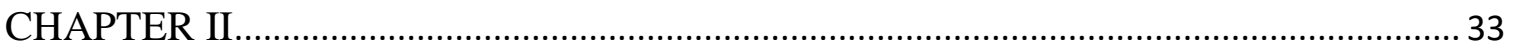

THIOL FUNCTIONALIZED GOLD NANOPARTICLE GAS SENSOR FOR VOC

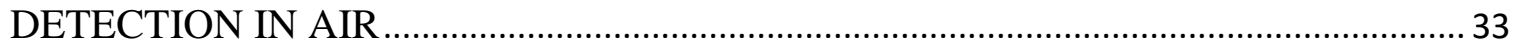

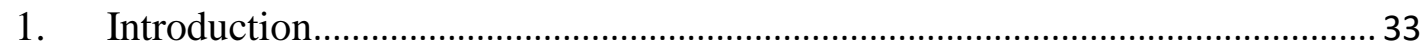

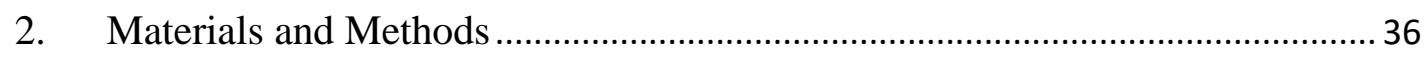

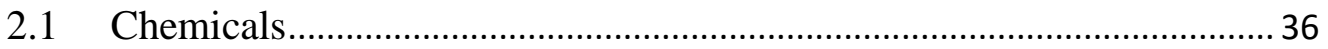

2.2 Synthesis of thiol functionalized gold nanoparticles (AuNPs) ...............36

2.3 Interdigitated electrodes (IDE) fabrication and AuNP film

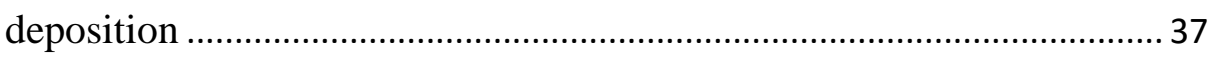

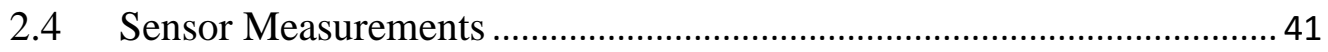

3. Material Structure and Characterization .................................................... 43

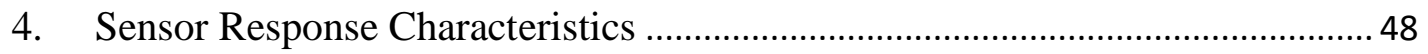

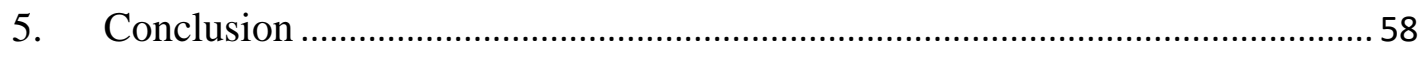

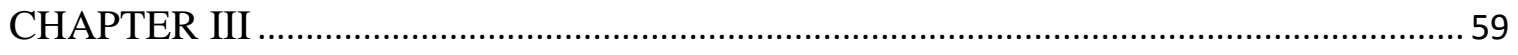

ACTUATING GOLD NANOPARTICLE CHEMIRESISTOR SENSITIVITY AND SELECTIVITY BY INCORPORATION OF A MOLECULAR RECOGNITION

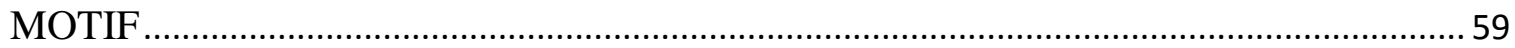

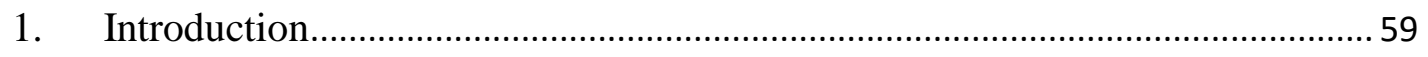

2. Synthesis of Gold Nanoparticles with Monoalkoxyurea Thiol 1 ...................... 61 
2.1 Materials and Methods.

2.2 Procedures for Synthesis of Monoalkoxyurea Thiol 1

2.3 Synthesis of Gold Nanoparticles Functionalized with Monoalkoxyurea

thiol 1. .65

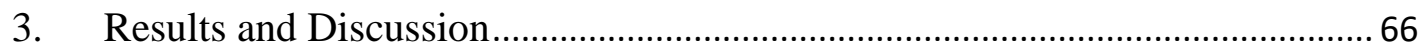

3.1 NMR Characterization of as-Synthesized Alkoxyurea Thiol 1 .66

3.1.1 ${ }^{1} \mathrm{H}$ and ${ }^{13} \mathrm{C}$ NMR of 2-(Undec-10-en-1-yloxy)isoindoline-1,3dione (2) .66

3.1.2 ${ }^{1} \mathrm{H}$ and ${ }^{13} \mathrm{C}$ NMR of 1-(tert-Butyl)-3-(undec-10-en-1-yloxy)urea (3).

3.1.3 ${ }^{1} \mathrm{H}$ and ${ }^{13} \mathrm{C}$ NMR of $S$-(11-((3-(tert-Butyl)ureido)oxy)undecyl) ethanethioate (4)..

3.1.4 ${ }^{1} \mathrm{H} \quad$ and $\quad{ }^{13} \mathrm{C} \quad \mathrm{NMR} \quad$ of 1 -(tert-Butyl)-3-((11mercaptoundecyl)oxy)urea (1)

3.2 Characterization of AuNP Functionalized with Monoalkoxyurea Thiol 1

3.3 VOC Sensing Properties of Alkoxyurea Thiol Gold Nanoparticles. 75

3.4 Long-term Stability of the Sensors

4. Conclusion .83

CHAPTER IV .85

\section{ELECTRONIC NOSE FOR DETECTION ACETONE IN EXHALED BREATH 85}

1. Introduction. .85

2. Materials and Methods .87 
2.1 Materials and Sampling.

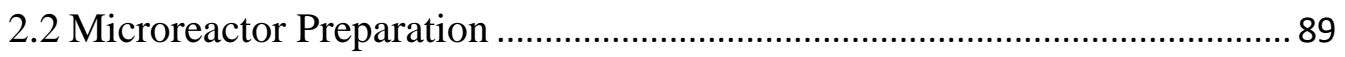

2.3 Evacuation of Exhaled Breath Samples Through Microreactors ................. 90

2.4 Analysis of Captured Carbonyls .................................................................... 92

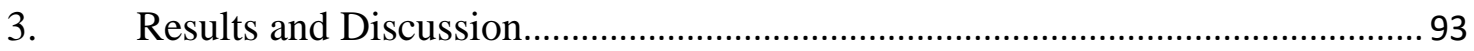

3.1 Humidity Effect on the Thiol Functionalized Gold Nanoparticle-Coated

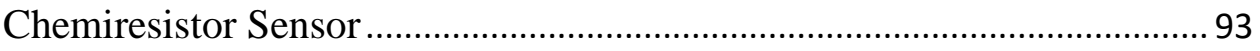

3.2 Breath Sample Storage Time............................................................................ 95

3.3 Determination of Acetone Concentration in Breath Samples by

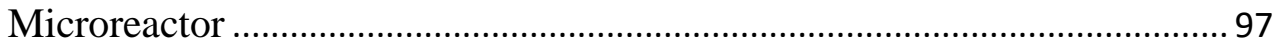

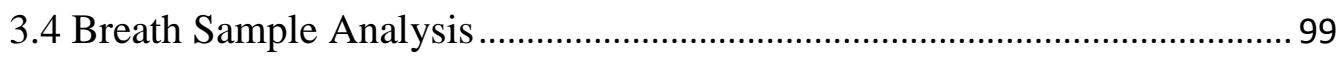

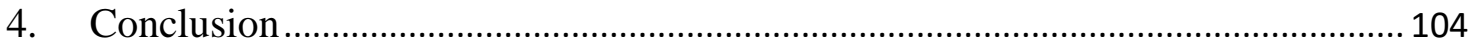

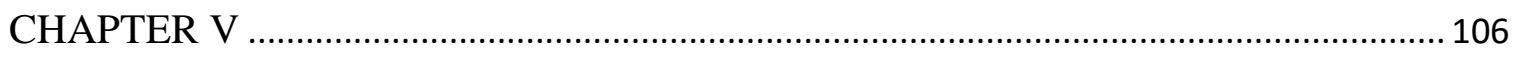

IMPROVING SENSITIVITY OF GOLD NANOPARTICLE SENSOR TOWARD

ACETONE BY DEVISING DIFFERENT FUNCTIONAL MOTIFS .............................. 106

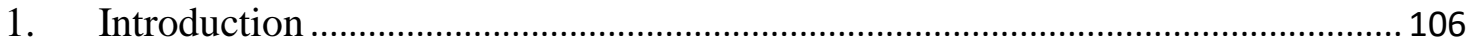

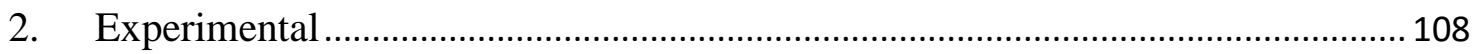

2.1 Procedures for Synthesis of Monoalkoxyurea Thiols .................................... 108

2.2 Procedures for Synthesis of Alkylurea Thiols ............................................ 112

2.3 Procedure for Synthesis of bis-Alkoxyurea Thiols ......................................... 116

2.4 Synthesis of Gold Nanoparticles (AuNPs) .................................................... 119

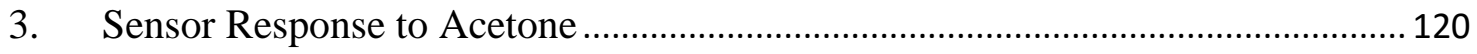

3.1 Response Results for Monoalkoxyurea Thiol Sensor Sensing Acetone ........ 121 
3.2 Response Results for Alkylurea Thiol Sensor Sensing Acetone

3.3 Response Results for bis-Alkoxyurea Thiol Sensor Sensing Acetone 130

4. Selectivity of tert-butyl Alkylurea Thiol Sensor for Acetone 134

5. Conclusions 135

CHAPTER VI

SUMMARY AND FUTURE WORK 137

1. Summary 137

2. Future Directions 139

REFERENCES . 142 CURRICULUM VITAE 168 


\section{LIST OF TABLES}

Table 1 Comparison of current widely-used medical diagnostic techniques and

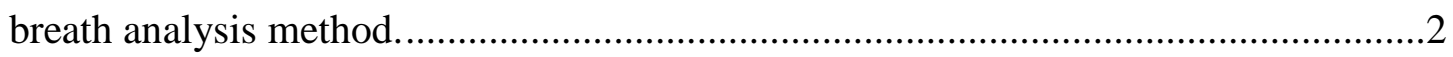

Table 2 Acetone in breath from diabetic patients and controls ..............................4

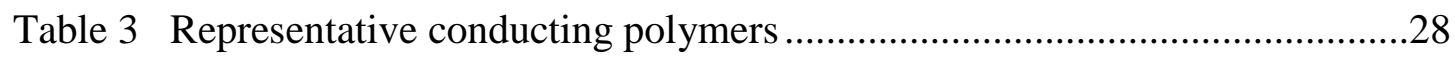

Table 4 Thiols used to functionalize the AuNPs chemiresistors .............................41

Table 5 Response sensitivities of different vapors on 5 thiol functionalized AuNP

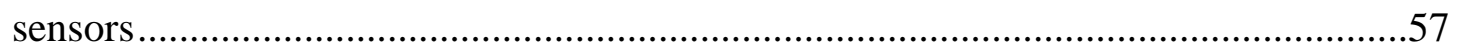

Table 6 A comparison of chemiresistor parameters and limits for detection of

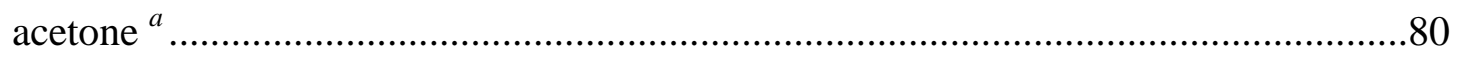

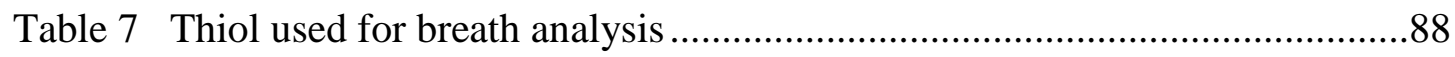

Table 8 Summary of acetone concentration in breath samples for different volunteers

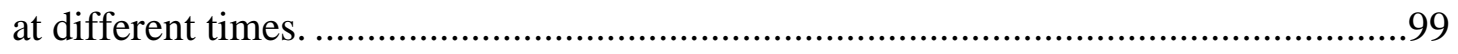

Table 9 Three series of urea thiol synthesized to functionalize gold nanoparticles 107 


\section{LIST OF FIGURES}

Figure 1.1 Gas Chromatograph/mass spectrometry (GC-MS) analysis of healthy and lung cancer breath...........................................5

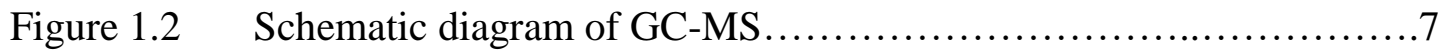

Figure $1.3 \quad$ Normal human breath chromatograph........................... 8

Figure 1.4 Schematic diagram of SIFT-MS ..............................

Figure 1.5 Schematic representation of the PTR-MS. N stands for neutral gas species, while SEM stands for secondary electron multiplier..........11

Figure 1.6 Schematic diagram of an ion mobility spectrometer................12

Figure 1.7 Schematic representation of a chemiresistive film placed on interdigitated electrodes................................................14

Figure 1.8 Schematic representation of a chemiresistive film of various dimensions comprised of metal monolayer-protected clusters (MPCs) separated by interdigitated SAM molecules..................................15

Figure 1.9 Conductance change of SWNT sensor when exposed to (A) $200 \mathrm{ppm}$ $\mathrm{NO}_{2},(\mathrm{~B}) 1 \% \mathrm{NH}_{3}$ gases......................................21

Figure 1.10 The electrical resistance between source and drain at $10 \mathrm{~V}$ bias voltage without any gate voltage under various N2 filling pressure from $50 \mathrm{~m}$ Torr to 500 Torr .22 
Figure 1.11 Sensor responses to $\mathrm{Hx}(\mathrm{A}), \mathrm{Bz}(\mathrm{B})$ and $\mathrm{Tl}(\mathrm{C})$ vapors. Left: response profiles for Hx $(413,826,1239,1652,2065,2478,2891,3304,3718$, and $4131 \mathrm{ppm},(\mathrm{A}))$; Bz $(260,519,779,1038,1298.0,1558,1817$, 2077, 2336, and 2590 ppm, (B)); and Tl (78, 155, 234, 310, 390, 466, 546, 621, 702, and 776 ppm, (C)). Right: corresponding response sensitivities .24

Figure 1.12 Illustration of the diagnosis of lung cancer using breath testing.......25

Figure 1.13 (A) A schematic of electrospinning process. (B) A SEM image of typical electrospun fibers........................................27

Figure 2.1 Schematic representation of thiol functionalized gold nanoparticle...34

Figure 2.2 IDE fabrication process flow diagram............................ 39

Figure 2.3 Microscopic image of the interdigital electrodes...................40

Figure 2.4 Sensor measurement set up diagram..........................42

Figure 2.5 (a) TEM micrograph of DDT functionalized AuNP and (b) histogram of AuNP size distribution......................................44

Figure 2.6 IR spectra of DDT and AuNP functionalized with DDT prepared in the present work............................................45

Figure 2.7 IR spectra of DDT and AuNP functionalized with DDT prepared in the

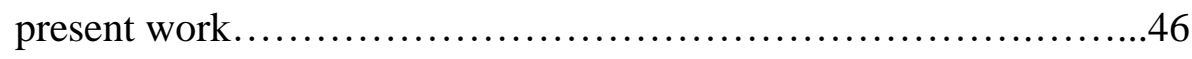

Figure 2.8 TGA of gold nanoparticles functionalized with (A) 1-dodecanethiol, and (B) 4-methoxy- $\alpha$-toluenethiol .47

Figure 2.9 (A) Sensor response profile for MTT AuNP film in response to ethanol vapor at the concentrations from $0.1 \mathrm{ppb}$ to $1000 \mathrm{ppm}$, (B) Sensor $x v i$ 
response versus $\log [$ analyte $(\mathrm{ppb})]$ in detection of acetone, ethanol, benzene and ethylbenzene

Figure 2.10 (A) Sensor response profile for MUA AuNP film in response to acetone vapor at the concentrations from $0.1 \mathrm{ppb}$ to $100 \mathrm{ppm}$, (B) Sensor response versus $\log [$ analyte $(\mathrm{ppb})]$ in detection of acetone, ethanol, benzene and ethylbenzene.

Figure 2.11 (A) Sensor response profile for DDT AuNP film in response to acetone vapor at the concentrations from $0.1 \mathrm{ppb}$ to $10 \mathrm{ppm}$, (B) Sensor response versus $\log [$ analyte $(\mathrm{ppb})]$ in detection of acetone, ethanol, benzene and ethylbenzene

Figure 2.12 (A) Sensor response profile for HDT AuNP film in response to acetone vapor at the concentrations from $0.1 \mathrm{ppb}$ to $1000 \mathrm{ppm}$, (B) Sensor response versus $\log [$ analyte $(\mathrm{ppb})]$ in detection of acetone, ethanol, benzene and ethylbenzene.

Figure 2.13 (A) Sensor response profile for ODT AuNP film in response to acetone vapor at the concentrations from $0.1 \mathrm{ppb}$ to $1000 \mathrm{ppm}$, (B) Sensor response versus $\log [$ analyte $(\mathrm{ppb})]$ in detection of acetone, ethanol, benzene and ethylbenzene.....................................55

Figure 2.14 Sensor responses to $0.1 \mathrm{ppm}$ VOCs...............................56

Figure 3.1 Synthesis procedure of monoalkoxyurea thiol.....................62

Figure 3.2 NMR spectra for 1H NMR and 13C NMR of 2 ..................67

Figure $3.3 \quad$ NMR spectra for H NMR and C NMR of $3 \ldots \ldots \ldots \ldots \ldots \ldots \ldots \ldots . \ldots 6$

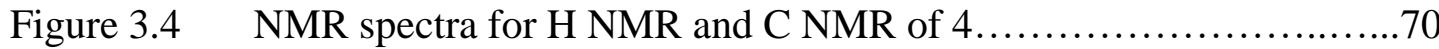
xvii 
Figure 3.5 NMR spectra for H NMR and C NMR of 1

Figure 3.6 A: Structure of thiol 1; B: SEM micrograph of the microfabricated, interdigitated electrode; $\mathrm{C}$ : size comparison between the sensor connected to platinum contact pads and a one-cent coin.

Figure 3.7 A: TEM of AuNP functionalized with thiol 1; B: histogram of AuNP size distribution .73

Figure 3.8 IR spectra of thiol 1 and thiol 1-functionalized AuNPs prepared in this work .74

Figure 3.9 TGA of AuNP with monoalkoxyurea thiol 1 .75

Figure 3.10 Response profile of thiol 1 AuNP sensor on repeated exposure to air, 1 ppb and $100 \mathrm{ppb}$ acetone. .76

Figure 3.11 A: Response of the thiol 1 AuNP sensor to acetone exposure at 0.1 ppb to $1000 \mathrm{ppm}$; B: sensor response versus $\log [$ analyte $(\mathrm{ppb})]$ in detection of acetone, ethanol, benzene, ethylbenzene and water vapor.

Figure 3.12 Responses for sensors derived from AuNPs coated with thiol urea 1, 1dodecanethiol 4-methoxy- $\alpha$-toluenethiol or 11-mercaptoundecanoic acid in detection of acetone. . .79

Figure 3.13 A comparison of the response sensitivities obtained for the four vapors on the four-sensor array system.

Figure 3.14 Responses of monoalkoxyurea thiol 1 AuNP sensor in detecting different carbonyl compounds. Error bars indicate the variability among three different sensors .82 
Figure 3.15 Stability of monoalkoxyurea thiol 1 sensor on exposure to $1 \mathrm{ppm}$ acetone over 30 days.................................... 83

Figure 4.1 Optical and SEM graphs of one microreactor......................90

Figure 4.2 Schematic flow diagram of the evacuation setup.................91

Figure 4.3 The reaction of DNPH with carbonyl compounds..................92

Figure $4.4 \quad$ Acetonitrile elution........................................ 92

Figure 4.5 Sensor responses to water vapor at relative humidity increased from

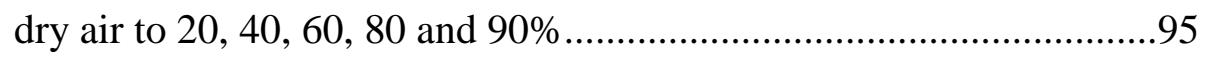

Figure 4.6 Stability of breath sample at $40{ }^{\circ} \mathrm{C}$ and room temperature for MTT senso.......................................................

Figure 4.7 Stability of breath sample at $40{ }^{\circ} \mathrm{C}$ and room temperature for DDT sensor.................................................. 96

Figure 4.8 Calibration curve for DNPH-acetone concentration vs peak area..................................................... 97

Figure 4.9 HPLC spectrum of one exhaled breath sample.....................98

Figure 4.10 (A) Responses of MTT AuNP sensor to breath sample with different concentrations $(0.1,0.5,1,2,3 \mathrm{ppm})$ of spiked acetone; (B) MTT AuNP sensor response versus acetone concentration in breath sample 101

Figure 4.11 (A) Responses of DDT AuNP sensor to breath sample with different concentrations $(0.1,0.5,1,2,3 \mathrm{ppm})$ of spiked acetone; (B) DDT AuNP sensor response versus acetone concentration in breath sample .102 
Figure 4.12 (A) Responses of monoalkoxyurea thiol 1 AuNP sensor to breath sample with different concentrations $(0.1,0.5,1,2,3 \mathrm{ppm})$ of spiked acetone; (B) urea thiol 1 AuNP sensor response versus acetone concentration in breath sample..............................103

Figure 5.1 Synthesis of monoalkoxyurea thiol............................ 108

Figure 5.2 Synthesis of alkylurea thiol................................ 112

Figure $5.3 \quad$ Synthesis of bis-alkoxy urea thiol............................116

Figure 5.4 Cyclohexyl monoalkoxyurea thiol sensor response profile in response to $100 \mathrm{ppb}$ and $1000 \mathrm{ppm}$ of acetone vapor......................121

Figure 5.5 Phenyl monoalkoxyurea thiol sensor response profile in response to $100 \mathrm{ppm}$ and $1000 \mathrm{ppm}$ of acetone vapor. .122

Figure 5.6 $p$-fluorophenyl monoalkoxyurea thiol sensor response profile in response to $0.1 \mathrm{ppb}$ to $10 \mathrm{ppm}$ of acetone vapor...................122

Figure 5.7 Hydrogen bonding interaction between AuNP with t-butyl monoalkoxyurea thiol and acetone..........................124

Figure $5.8 \quad$ (A) tert-butyl alkylurea thiol sensor response profile in response to 0.1 ppb to $1000 \mathrm{ppm}$ of acetone vapor. (B) Sensor response versus $\log [$ acetone $(\mathrm{ppb})]$ in detection of different concentrations of acetone. .125

Figure 5.9 (A) Cyclohexyl alkylurea thiol sensor response profile in response to 0.1 ppb to $1000 \mathrm{ppm}$ of acetone vapor. (B) Sensor response versus $\log [$ acetone $(\mathrm{ppb})]$ in detection of different concentrations of acetone 126 
Figure $5.10 \quad$ (A) Phenyl alkylurea thiol sensor response profile in response to 0.1 ppb to $1000 \mathrm{ppm}$ of acetone vapor. (B) Sensor response versus $\log [$ acetone $(\mathrm{ppb})]$ in detection of different concentrations of acetone........................................................ 127

Figure 5.11 (A) $p$-fluorophenyl alkylurea thiol sensor response profile in response to $0.1 \mathrm{ppb}$ to $1000 \mathrm{ppm}$ of acetone vapor. (B) Sensor response versus $\log [$ acetone $(\mathrm{ppb})]$ in detection of different concentrations of acetone.................................................. 128

Figure 5.12 (A) Tert-butyl bis-alkoxyurea thiol sensor response profile in response to $0.1 \mathrm{ppb}$ to $1000 \mathrm{ppm}$ of acetone vapor. (B) Sensor response versus $\log [$ acetone $(\mathrm{ppb})]$ in detection of different concentrations of

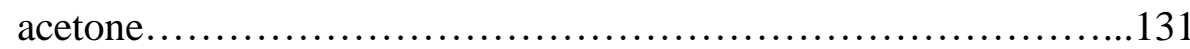

Figure $5.13 \quad$ (A) Cyclohexyl bis-alkoxyurea thiol sensor response profile in response to $0.1 \mathrm{ppb}$ to $1000 \mathrm{ppm}$ of acetone vapor. (B) Sensor response versus $\log [$ acetone $(\mathrm{ppb})]$ in detection of different concentrations of acetone.................................... 132

Figure 5.14 The response patterns for three series urea thiol sensors sensing $1 \mathrm{ppm}$ acetone. The inserted graph is a magnified view of the response below 6. 134

Figure 5.15 Tert-butyl alkylurea thiol sensor response versus log[analyte (ppb)] in detection of acetone, ethanol and benzene. .135 


\section{CHAPTER I}

\section{INTRODUCTION}

\section{Breath Analysis}

Breath analysis has the potential to be a diagnostic method that links specific volatile organic compounds (VOCs) in exhaled breath to medical conditions $[1,2]$, and offers several advantages over other traditional diagnostic techniques that involve analysis of blood, urine, biopsy, endoscopy, and imaging. It could potentially provide a fast, complete non-invasive and painless means to access a person's state of health, and virtually limitless repeatability with respect to frequency, access and cost. Even though up to 3000 compounds may be detected in breath, the matrix of exhaled air is still less complex than that of blood or other body fluids. Also, it can be used on all people, regardless of age, gender, race, disability or other characteristic. Table I compares the current widely-used medical diagnostic techniques with breath analysis in these aspects [3]. All these medical diagnostic techniques suffer from invasiveness and/or practical restrictions on repeatability. Breath analysis, if it fully realizes its potential, will suffer from neither, enabling revolutionary diagnostic applications. However, the practical repeatability of breath analysis is limited by factors such as 
sample collection, cost, user-friendliness, and real-time measurement capability. For example, patients cannot come to a clinic daily to have their breath analyzed, as this would be very inconvenient and expensive in comparison to the existing alternative. A successful monitoring program using breath analysis would require an inexpensive, in-home, self-administered test.

Table 1 Comparison of current widely-used medical diagnostic techniques and breath analysis method. (Reprinted from [3]: P.X. Braun, et al., 2012)

\begin{tabular}{|c|c|c|c|c|c|c|c|c|}
\hline & \multirow{2}{*}{ Bicod } & \multirow{2}{*}{ Urine } & \multirow{2}{*}{ Bicpsy. } & \multirow{2}{*}{ Endoscopy } & \multicolumn{3}{|c|}{ Examples of imaging } & \multirow{2}{*}{$\begin{array}{l}\text { Breath } \\
\text { Analysis }\end{array}$} \\
\hline & & & & & $X-r a y i C T$ scan & MRI & Sonography & \\
\hline \multicolumn{9}{|l|}{$\begin{array}{l}\text { Non- } \\
\text { invasive? }\end{array}$} \\
\hline \multicolumn{9}{|c|}{$\begin{array}{l}\text { Limitlossly } \\
\text { repeatable? }\end{array}$} \\
\hline Reason & $\begin{array}{l}\text { Invasive: } \\
\text { painful } \\
\text { Non- } \\
\text { repeatabie: } \\
\text { Anise. } \\
\text { expensa, } \\
\text { logistics }\end{array}$ & $\begin{array}{l}\text { Nan- } \\
\text { cepeacable: } \\
\text { finte, } \\
\text { timing. } \\
\text { expense, } \\
\text { logistes }\end{array}$ & $\begin{array}{l}\text { Highiy } \\
\text { mvasive } \\
\text { Non- } \\
\text { repeatabie: } \\
\text { magcr } \\
\text { procedure. } \\
\text { expense }\end{array}$ & $\begin{array}{l}\text { Invavive } \\
\text { procedure } \\
\text { Non- } \\
\text { repeatable: } \\
\text { major } \\
\text { proceduru, } \\
\text { expense. }\end{array}$ & $\begin{array}{l}\text { Invasive: } \\
\text { iocising radiation } \\
\text { Non-apeatable: } \\
\text { radiation } \\
\text { axposure, } \\
\text { oxpense, } \\
\text { logistics }\end{array}$ & $\begin{array}{l}\text { Possibly } \\
\text { invassive: } \\
\text { injected dyes } \\
\text { Non- } \\
\text { repeatable: } \\
\text { expensa. } \\
\text { logistics }\end{array}$ & $\begin{array}{l}\text { Non. } \\
\text { repeatable: } \\
\text { expense. } \\
\text { iogistics }\end{array}$ & \\
\hline
\end{tabular}

The detection of VOCs in breath for diagnosing disease dates back to ancient times. For instance, the ancient Greek physician Hippocrates reported that some particular diseases could be diagnosed from the distinct odors [4]. For example, uncontrolled diabetes can produce acetone which has a sweet smell, liver diseases produce a fishy smell, while kidney failures result in a urine-like smell [5, 6]. Modern breath analysis began in 1971 when Pauling demonstrated that human breath is a complex gas, containing more than 200 different VOCs in exhaled breath, measured by gas chromatography [7]. In the last 3 decades, the problems of separation and 
identification of these compounds have been solved due to the technical progress of analytical methods. More recently, the issues concerning the correlations of specific VOCs in exhaled breath with medical conditions have gained great interest [8]. In the 1990s, some researchers found a correlation between nitric oxide and asthma [9-11]. Since then, many other researchers have tried to correlate diseases with particular breath biomarkers, from renal failure to cancer [12-15].

A biomarker refers to a traceable substance, which indicates the existence of a living organism, as a means to examine organ function or other aspect of health. For example, acetone is a biomarker of diabetes $[16,17]$. Acetone concentrations higher than $1.71 \mathrm{ppm}$ are common in diabetic patients' breath, while concentrations lower than $0.76 \mathrm{ppm}$ is normal in healthy human breath. Table 2 shows the acetone concentrations in 15 diabetic patients and 15 controls as measured by an external standard method [18]. 
Table 2 Acetone in breath from diabetic patients and controls (Reprinted from [18]:

C. Deng, et al., 2004).

\begin{tabular}{llll}
\hline Patient & $\begin{array}{l}\text { Acetone } \\
\text { concentration (ppmv) }\end{array}$ & Control & $\begin{array}{l}\text { Acetone } \\
\text { concentration (ppmv) }\end{array}$ \\
\hline 1 & 1.87 & 1 & 0.48 \\
2 & 1.81 & 2 & 0.55 \\
3 & 2.19 & 3 & 0.23 \\
4 & 2.26 & 4 & 0.52 \\
5 & 2.03 & 5 & 0.66 \\
6 & 2.44 & 6 & 0.70 \\
7 & 2.50 & 7 & 0.44 \\
8 & 2.78 & 8 & 0.40 \\
9 & 3.73 & 9 & 0.75 \\
10 & 2.30 & 10 & 0.31 \\
11 & 1.88 & 11 & 0.22 \\
12 & 3.30 & 12 & 0.48 \\
13 & 1.76 & 13 & 0.80 \\
14 & 2.40 & 14 & 0.59 \\
15 & 2.07 & 15 & 0.54 \\
\hline
\end{tabular}

Peng et al. identified the VOCs that can serve as biomarkers for lung cancer in the breath samples and determined their relative concentrations [19]. Figure 1.1 shows the concentration levels of VOCs exhaled from lung cancer subjects compared to healthy subjects [19]. 


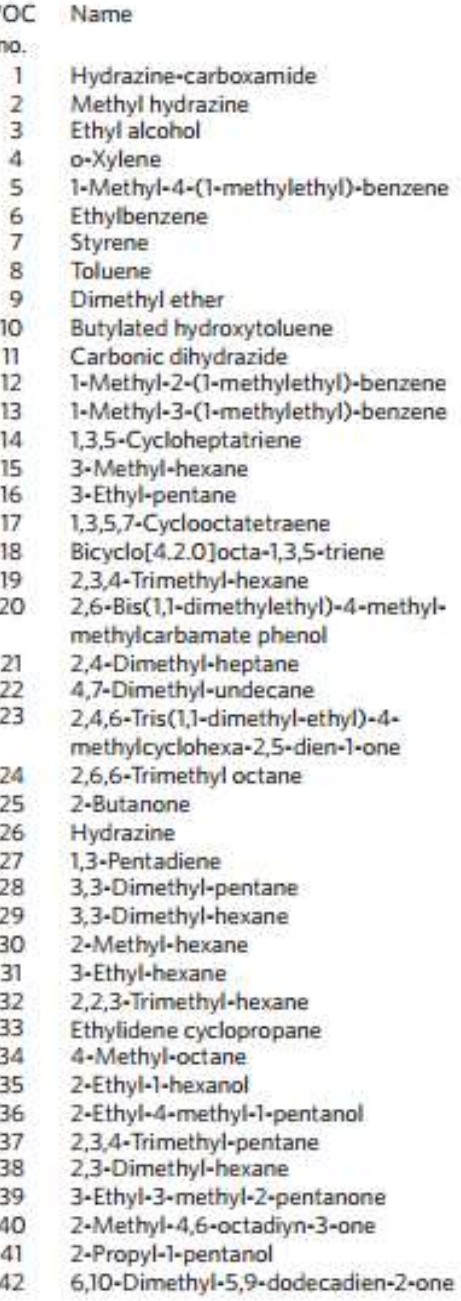

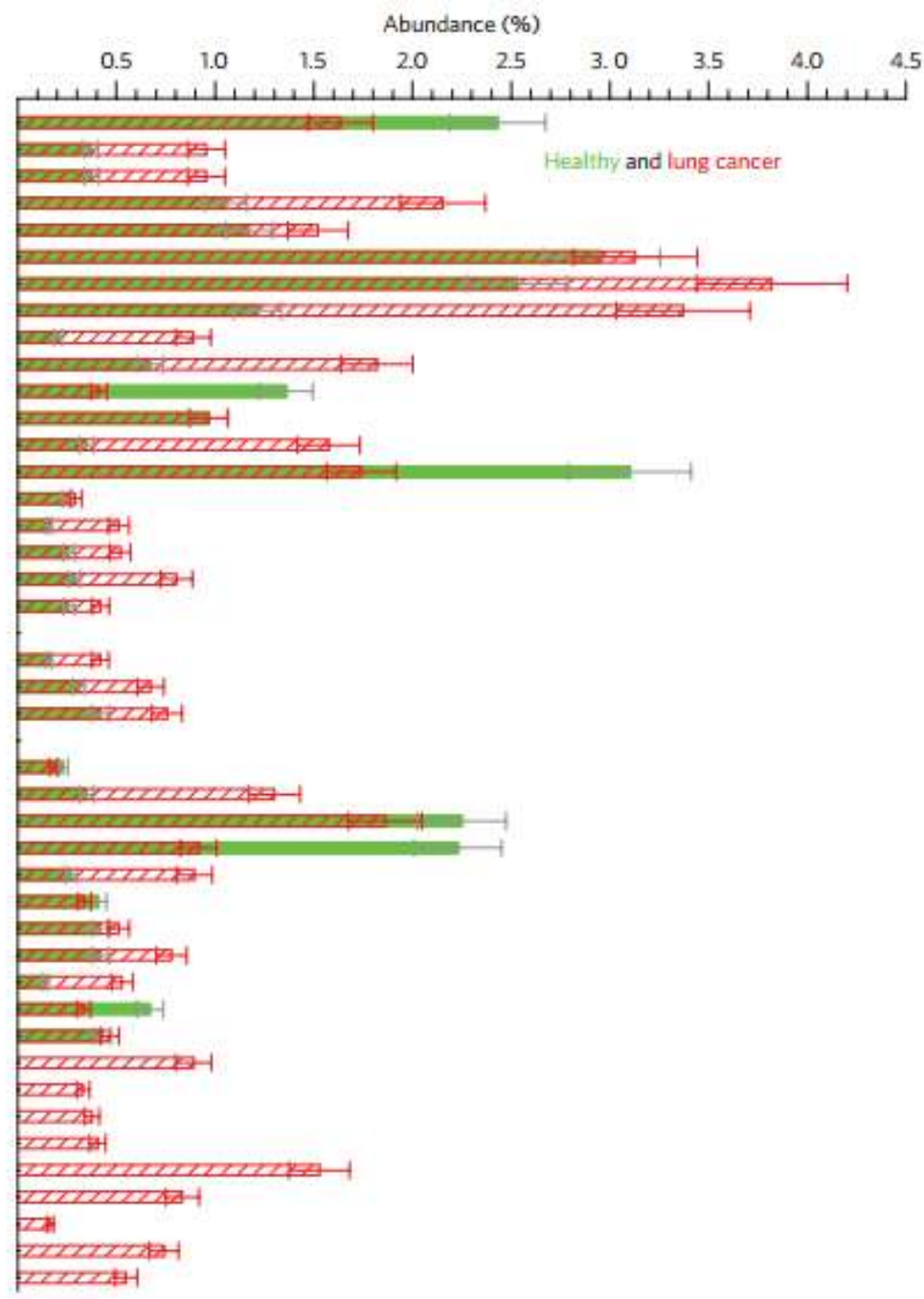

Figure 1.1 Gas Chromatograph/mass spectrometry (GC-MS) analysis of healthy and lung cancer breath (Reprinted from [19]: G. Peng et al., 2009).

The VOC analytical methods can be classified into two categories: One is based on gas chromatography (GC) or mass spectrometry (MS) and the other one relies on use of an electronic nose. 


\section{GC or MS Based Techniques for VOC Detection}

As the most commonly used method, GC and MS have been coupled to various sample collection methods. Among all the developed methods for VOCs, GCMS is one of the most widely employed techniques for VOC detection and breath analysis. However, these methods are commonly compatible with sample preconcentration process, such as cold-trap or adsorption trap method because of the low concentration of VOCs in breath samples [20, 21]. Solid-phase microextraction (SPME) is one of the most advanced sampling techniques for GC analysis [18, 22, 23], which has some advantages over traditional extraction methods, such as simple sample collection, no solvent use and easy automation.

\subsection{Gas Chromatography-Mass Spectrometry (GC-MS)}

Figure 1.2 shows the schematic diagram of GC-MS. The sample is first injected into the GC system and then the molecules in the mixture are separated as the sample travels the length of the column based on the difference in the chemical properties between different molecules and their relative affinity for the stationary phase of the column. The molecules are introduced to mass spectrometry to capture, ionize, accelerate, deflect and detect the ionized molecules separately. The MS breaks each molecule into ionized fragments and characterizes the fragments by their massto-charge ratio. There are several types of mass spectrometer detectors that can be used in GC-MS, including tandem quadrupoles (MS-MS), quadrupole mass 
spectrometer and ion trap mass spectrometer[24, 25]. Ion trap is more sensitive than quadrupole. The ions are produced by an electron beam in an ion source, stored in the trap and rejected according to their $\mathrm{m} / \mathrm{z}$ ratio to obtain a spectrum. However, the major disadvantage is that the spectra are often modified by self-chemical ionization and collision-induced dissociation. So the interpretation of spectrum is difficult, especially for polar compounds such as aldehydes, alcohols, and ketones etc., which are important compounds in exhaled breath analysis. Wiley and McLaren described time-of-flight (TOF) MS as another kind of mass spectrometer in 1955 [24]. The TOF instrument is able to detect specific compounds related to the disease status and can identify the unique VOCs at the same time. Therefore, it has been suggested that GCTOF-MS is preferred to be used as an excellent screening tool for new biomarkers in the monitoring for exposures and diseases [13, 26-28].

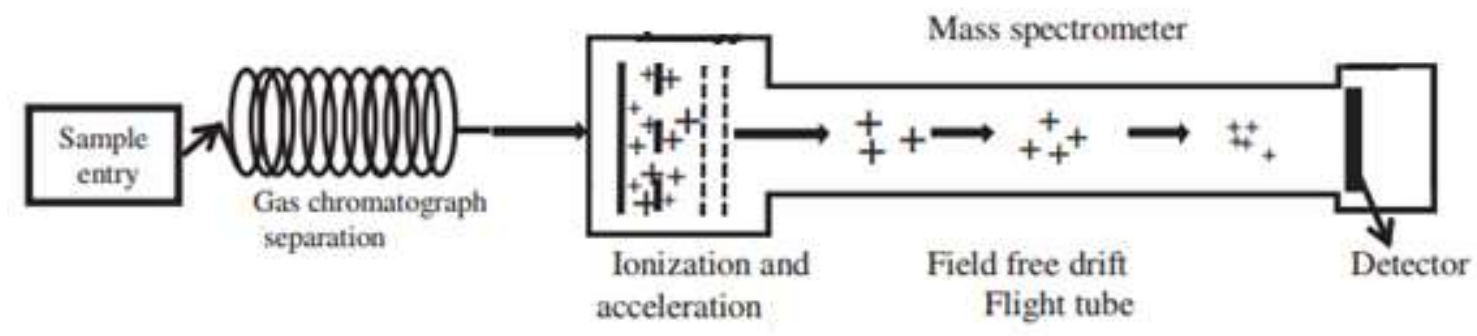

Figure 1.2 Schematic diagram of GC-MS (Reprinted from [26]: A.W. Boots, et al.)

GC-MS technique provides a reliable and highly sensitive method for VOC analysis, and offers highly reproducible results. Figure 1.3 shows the chromatograph of healthy human breath for the identification of metabolic end products pentane, acetone, ethanol, isoprene and other VOCs [13]. However, there are a number of 
limitations of GC-MS technique. It requires not only breath adsorption devices and column calibration for desired analytes, but also sampling and preconcentration steps prior to the injection of the sample into a GC column, which can lead to losses of analytes and contamination problems. The measurements are slow, expensive and currently immobile. Therefore, it is not possible for real time measurement [29-31]. Besides, GC-MS is less preferable to quantify some important trace compounds that are present in exhaled breath.

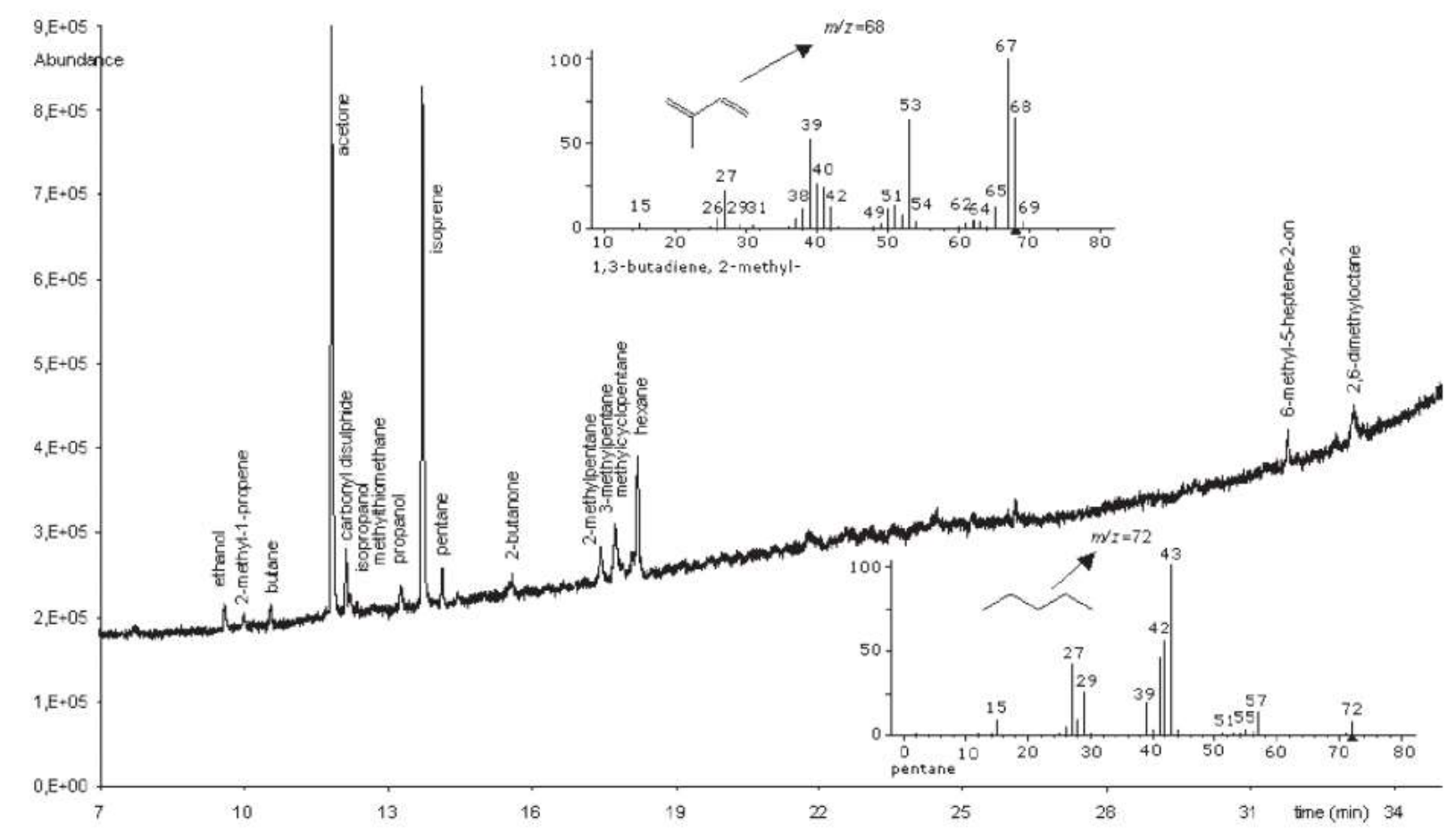

Figure 1.3 Healthy human breath chromatograph (Reprinted from [13]: B. Buszewski, et al., 2007) 


\subsection{Selected Ion Flow Tube Mass Spectrometry (SIFT-MS)}

SIFT-MS is a quantitative analytical technique for use in human breath analysis, and is of great interest as a non-invasive tool for physiological monitoring and disease diagnosis because it offers real time VOC quantification [32, 33]. Figure 1.4 shows the schematic diagram of SIFT-MS. In SIFT-MS, precursor ions $\left(\mathrm{H}_{3} \mathrm{O}^{+}\right.$, $\mathrm{NO}^{+}$and $\mathrm{O}_{2}^{+}$) are produced in a microwave plasma ion source, usually from a mixture of moist atmospheric air and corona discharge. The specific precursor ions are selectively separated by the first MS process and then react with the analyte molecules coming from the breath sample. The newly formed product ions can be analyzed by the second downstream quadrupole MS and detected. Therefore, quantification of particular trace gases in the breath sample is achieved.

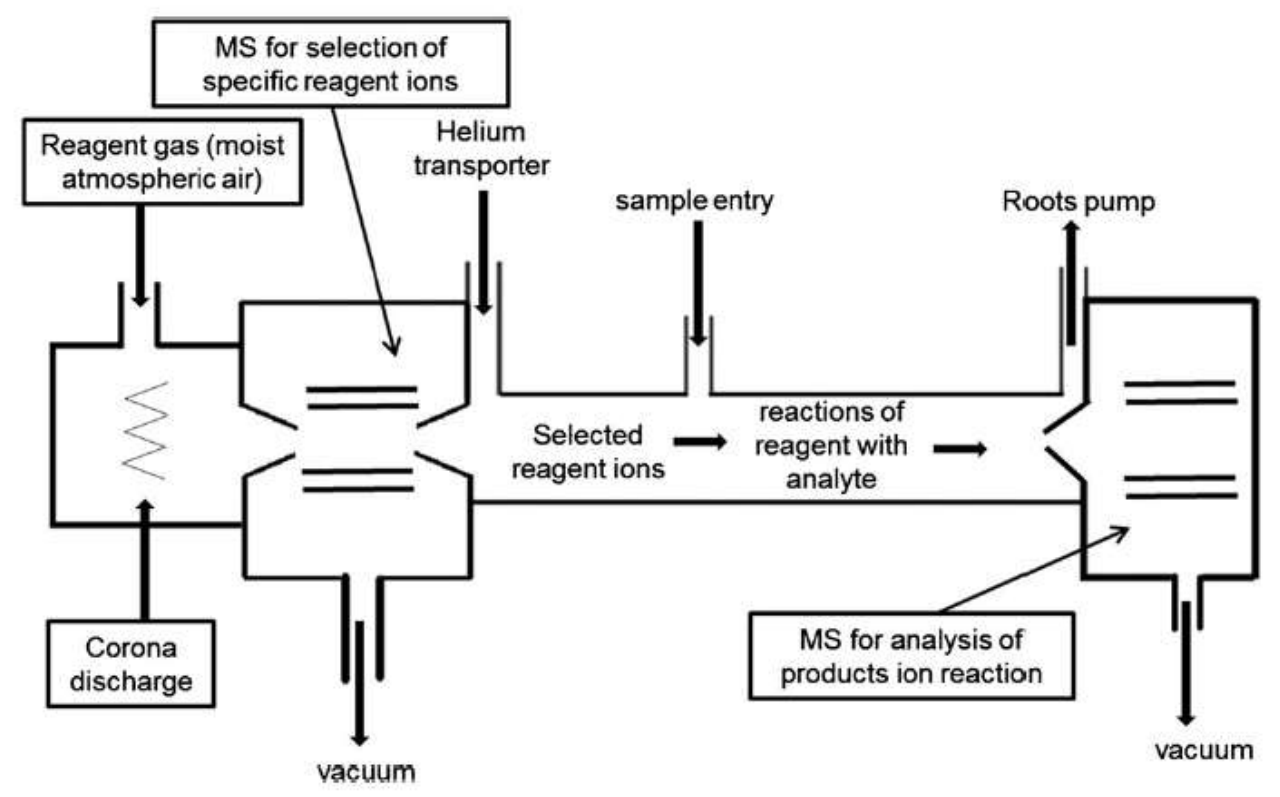

Figure 1.4 Schematic diagram of SIFT-MS (Reprinted from [26]: A.W. Boots, et al., 2012) 
SIFT-MS has several advantages, including high sensitivity, low detection limits (ppb level), short response time, quantification of water vapor and no need for sample preconcentration. Kumar et al. investigated a total of 17 VOCs by SIFT-MS in 2013 and discriminated the esophago-gastric cancer cohort from positive controls by 4 VOCs (hexanoic acid, phenol, methyl phenol and ethyl phenol) [34]. However, this technique is expensive and it cannot identify the whole breath-print because it can only detect gases for which positive precursor ions are selected [35]. In addition, the identification of VOCs present in breath samples based only on the mass-tocharge ratios of chemically ionized molecular ions is difficult.

\subsection{Proton Transfer Reaction-Mass Spectrometry (PTR-MS)}

PTR-MS is used for online monitoring of VOCs in complex gas mixtures based on chemical ionization of the target molecules by proton transfer reactions. The technique was developed by Hansel et al. in 1995 [36, 37]. In this method, a hollow cathode was exposed to water vapor to generate reagent ions $\left(\mathrm{H}_{3} \mathrm{O}^{+}\right)$with high purity. The ions from the source drift region are drawn by an electric field into a drift tube. In the drift tube, the proton transfer reactions in moist air between $\mathrm{H}_{3} \mathrm{O}^{+}$and any molecule whose proton affinity exceeds that of water. The ions then reach the MS detector.

PTR-MS has high specificity and high sensitivity (down to parts per trillion) and there is no need for sample preconcentration or separation processes, so VOCcontaining air and fluids headspaces can be analyzed directly. Also, it enables real- 
time measurement of samples, with a typical response time of $100 \mathrm{~ms}$. VOCs can be monitored online, which is very important in situations where rapid and sudden changes of VOC concentrations are expected [38]. Moreover, PTR-MS can give more reliable quantitative results. Absolute concentrations are obtained directly without previous calibration measurements. In 2007, Wehinger et al. analyzed VOCs in exhaled breath samples using PTR-MS for the detection of lung cancer [39]. Unfortunately, proton transfer from $\mathrm{H}_{3} \mathrm{O}^{+}$is not suitable for all fields of application, because only molecules with a proton affinity higher than water can be detected by PTR-MS [40, 41]. It is also not applicable for concentrated samples as the total concentration of VOCs must not exceed 10 ppmv since it is based on the assumption that the decrease of primary ions is negligible.

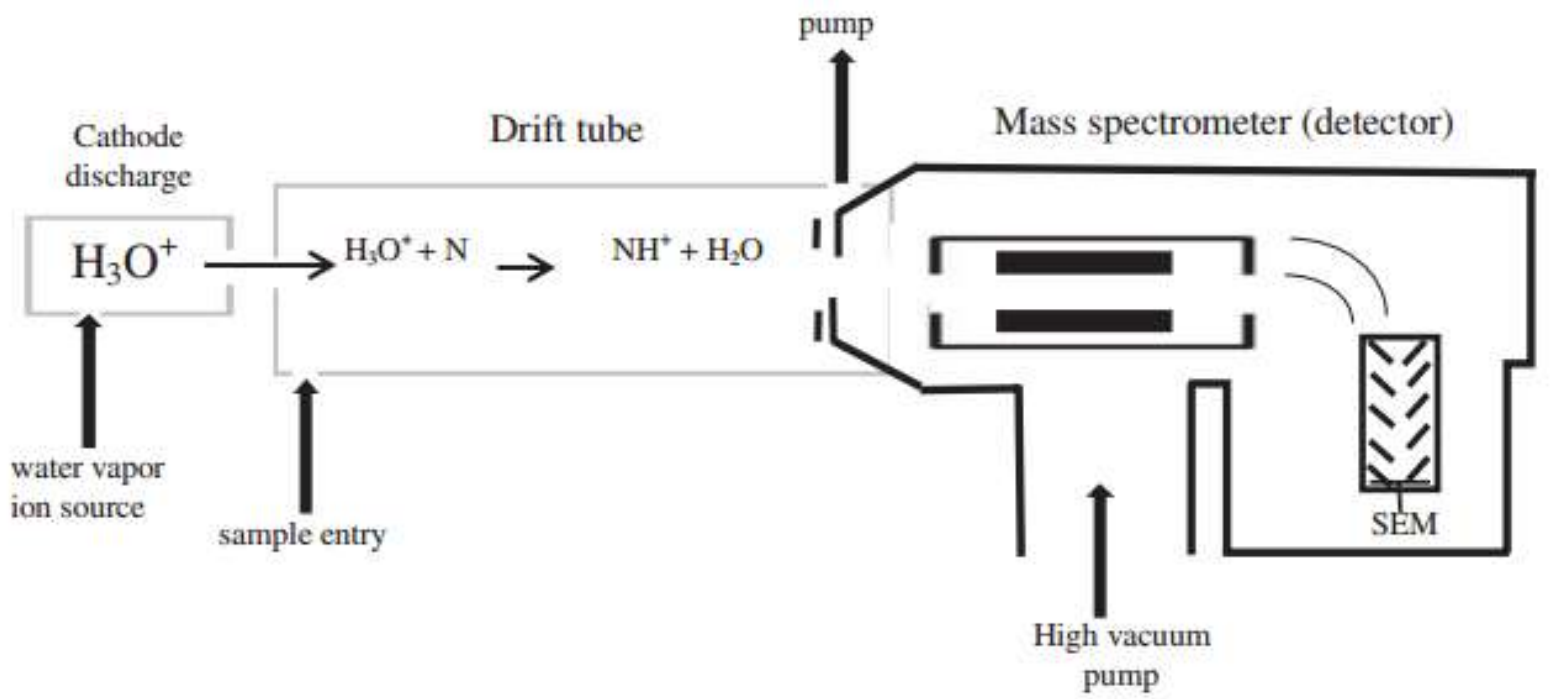

Figure 1.5 Schematic representation of the PTR-MS. N stands for neutral gas species, while SEM stands for secondary electron multiplier. (Reprinted from [26]: A.W. Boots, et al., 2012) 


\subsection{Ion Mobility Spectrometry (IMS)}

Ion mobility spectrometry (IMS) is an analytical instrumental technique used to separate and identify ionized molecules in the gas phase according to their mobility in a carrier buffer gas as they move through a drift tube filled with a purified gas such as air or nitrogen $[42,43]$. As shown in Figure 1.6, sample molecules are first ionized and then drift in the flight tube. The ions are distinguished as a result of the difference in the mas, size, shape and charge, and then the velocity is influenced by both the electric field and drift gas. Small ions (a) travel faster than heavier ions (b and c). Then they reach a detector plate and the signal is obtained.

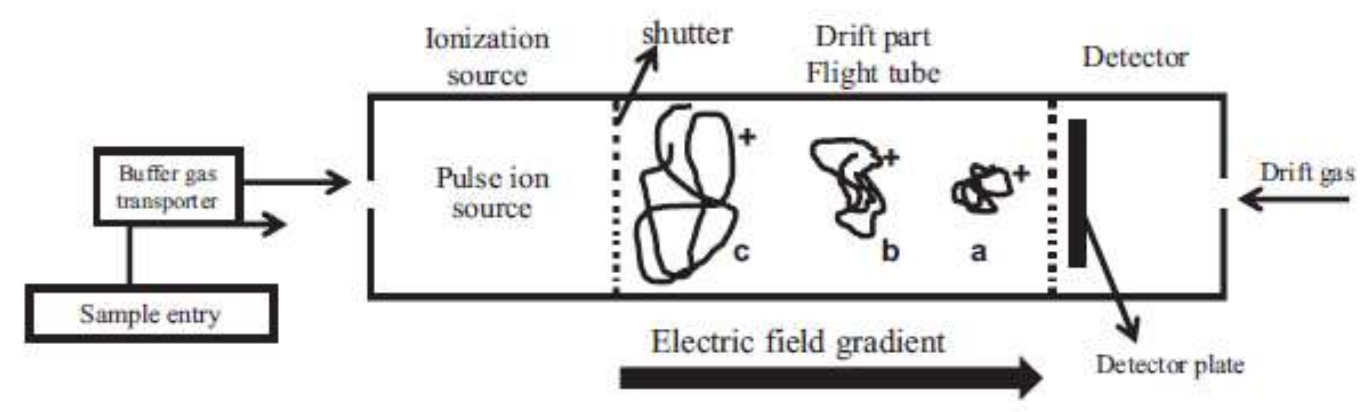

Figure 1.6 Schematic diagram of an ion mobility spectrometer. (Reprinted from [26]:

A.W. Boots, et al., 2012)

IMS alone is insufficient to identify an unknown compound, and it is also not suitable for real-time measurements. So IMS is often coupled with GC, LC or MS, 
which overcomes several limitations present in simple IMS systems [44]. Recently, IMS has been applied for breath analysis, which was reliable, rapid and cost-effective [45].

GC/MS based techniques give information about VOCs but the correlation between analytical results and actual odor perception is not direct due to potential interactions between several odorous components.

\section{Chemiresistors}

Chemiresistors are a class of chemical sensor that changes its electrical resistance in the presence of a chemical analyte of interest. A schematic of a chemiresistor is shown in Figure 1.7. The gas sensor consists of a chemically sensitive conductive film deposited onto the electrode. Upon application of a potential $(\mathrm{V})$, there is a measurable current or resistance, $\mathrm{R}_{0}$, across the film that changes to $\mathrm{R}$ upon interaction with an analyte of interest. There are several types of chemiresistor sensors using different sensing materials, including metal oxide nanowires [46-50], metal oxide composites [51-59], carbon nanotubes [60, 61], semiconductors [62], polymer nanofibers [63, 64], and nanoparticle-based materials [65] for detection of acetone as well as a plethora of other VOCs $[66,67]$. 

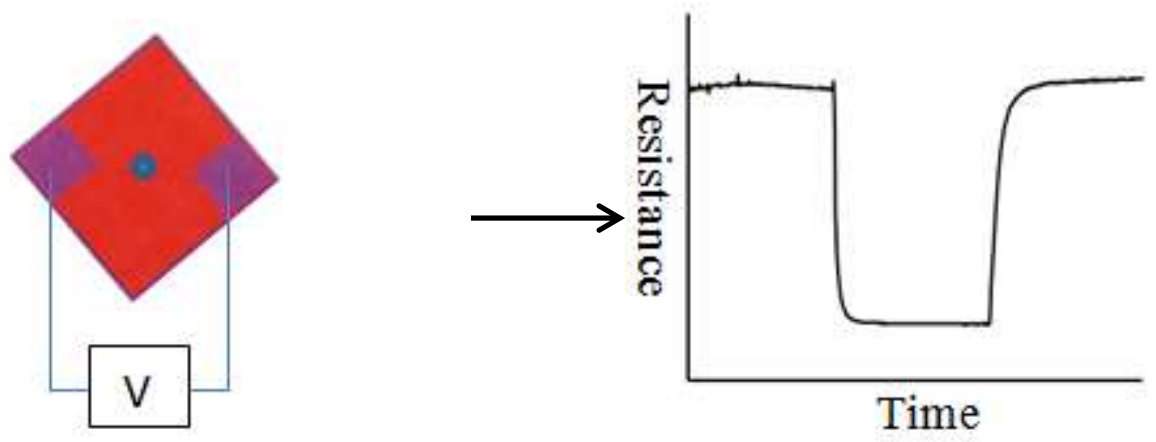

Figure 1.7 Schematic representation of a chemiresistive film placed on interdigitated electrodes. Resistance is monitored over time by the presence of an analyte. The adsorption and desorption processes are reversible, and the resistance is able to return to its original value as the compound leaves the sensing film.

The chemical interaction between the sensing material and the analyte can be by covalent bonding, hydrogen bonding, or molecular recognition. An Analyte diffuses into the films at a proportion defined by its partition coefficient and this characterizes the sensitivity and selectivity of the chemiresistor material. The conductivity of the films of ligand modified GNP has been described as an activated core-to-core electron hopping mechanism by the following equation:

$\sigma_{\mathrm{EL}}=\mathrm{o}_{0} \exp \left[-\beta_{\mathrm{d}} \delta_{\text {edge }}\right] \exp \left[-\mathrm{E}_{\mathrm{A}} / \mathrm{RT}\right]$

where o ${ }_{\mathrm{EL}}$ is electronic conductivity $\left(\Omega^{-1} \mathrm{~cm}^{-1}\right), o_{0}$ is a pre-exponential constant, $\delta_{\text {edge }}$ is the core edge-to-edge distance, $\beta_{\mathrm{d}}$ is the electron tunneling coefficient $\left(\AA^{-1}\right), \mathrm{R}$ is the gas constant, and $\mathrm{T}$ is the temperature $(\mathrm{K}), \mathrm{E}_{\mathrm{A}}$ is the activation energy for electron transfer $\left(\mathrm{kJ} \mathrm{mol}^{-1}\right)$, which is described as follows: 
Where e is the charge of an electron, $\varepsilon_{0}$ and $\varepsilon_{\mathrm{r}}$ represent the vacuum permittivity and relative permittivity of the dielectric medium surrounding the metal nanoparticle, respectively, and $\mathrm{r}$ is the radius of the nanoparticles.

Figure 1.8 shows monolayer-protected clusters separated by interdigitated self-assembled monolayer (SAM) molecules through which electron hopping occurs [68].

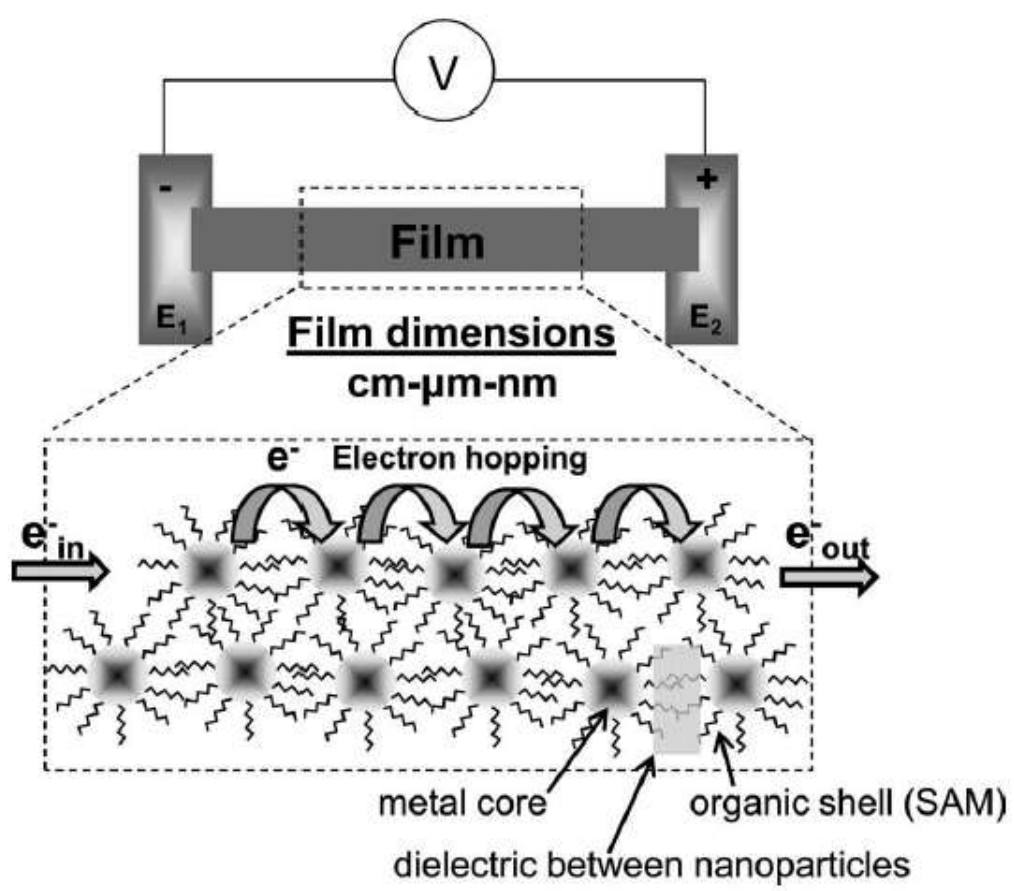

Figure 1.8 Schematic representation of a chemiresistive film of various dimensions comprised of metal monolayer-protected clusters (MPCs) separated by interdigitated 
SAM molecules. Electron hopping occurs from core to core when a voltage $(\mathrm{V})$ is applied. (Reprinted from [68]: F.J. Ibanez, F.P. Zamborini, 2012)

There are two important points that need to be noted regarding equation (1) and (2):

(a) Conductivity is exponentially dependent on the core edge-to-edge distance, $\delta$ and the tunneling coefficient, $\beta_{\mathrm{d}}$, which decreases with increasing particle core distance. $\beta_{\mathrm{d}}$ usually referred to as an attenuation factor describing the exponential decay in electron transfer as the distance between the nanoparticle cores increases, depends on the structure and molecular composition of the self-assembled molecules.

(b) The activation energy is inversely proportional to the relative permittivity of the media and the radius of the core. Thus an increase in the permittivity of the organic matrix surrounding the metal cores increases the conductivity due to the decrease in the activation energy, $\mathrm{E}_{\mathrm{A}}$.

Equation 2 shows that $\mathrm{E}_{\mathrm{A}}$ is inversely proportional to $\varepsilon_{\mathrm{r}}$. A decrease in $\varepsilon_{\mathrm{r}}$ will lead to an increase in $\mathrm{E}_{\mathrm{A}}$ and a corresponding exponential increase in $\mathrm{O}_{\mathrm{EL}}$. In general, $\sigma_{E L}$ from Equation 1 will change if an analyte of interest partitioning into the film of nanoparticles causes a change in $\delta_{\text {edge }}$ through film swelling or $E_{A}$ and $\beta_{\mathrm{d}}$ by significantly altering the dielectric properties surrounding the monolayer. For sensing experiments at room temperature, a change in all three variables will affect the conductivity and it is often not known which variable is the dominant factor. 


\section{Electronic Nose for VOC detection}

Beside of classical sample analysis techniques, electronic noses have been developed for exhaled breath VOC analysis. An electronic nose consists of a sample delivery system, a detection system and a computing system. The devices are commonly inexpensive, portable and amenable to use in widespread screening. In most electronic noses, each sensor is sensitive to all volatile molecules but each in a specific way. Most electronic noses use sensor arrays that react with VOCs on contact: the adsorption of VOCs on the sensor surface causes a change in the properties of the sensor. The most commonly used sensors for electronic nose include metal oxide semiconductor sensors, nanomaterial sensors, conducting polymer sensors, piezoelectric sensors, and colorimetric sensors.

\subsection{Metal Oxide Semiconductor Sensors}

Recently, metal oxide semiconductor sensors have been widely investigated for VOC analysis and utilized in a variety of different roles and industries [69-72]. Metal oxide semiconductor gas sensor devices have several advantages, such as low cost, small size, measurement simplicity, durability, ease of fabrication and low limits of detection (<ppm levels). Moreover, most metal oxide semiconductor based sensors tend to be stable and somewhat resistant to poisoning. Thus, they have become a prime technology in several domestic, commercial and industrial gas sensing systems. 
These sensors can react with the target VOCs whereby the metal oxide undergoes reduction and oxidation, leading to exchange of electrons at a certain characteristic rate, thereby affecting the sensor's resistance and yielding a certain signal $[73,74]$. Therefore, the sensing materials have to be carefully selected and modified, and the sensor film needs to be properly deposited in order to enhance the sensitivity and selectivity of the reaction of the target VOCs, to shorten the response time, and to lower the operation temperature $[75,76]$. One approach to do this is to obtain a high efficiency for the catalytic reactions at the sensor surface [77].

In 1995, Barbi et al. developed a $\mathrm{SnO}_{2}$ based sensor that showed a response to the presence of CO gas from concentrations of $10 \mathrm{ppm}$ and above [78]. In 2009, Li et al. compared different phases of titanium oxide $\left(\mathrm{TiO}_{2}\right)$ films [79]. Anatase $\mathrm{TiO}_{2}$ was found to show an n type response to the presence of CO. In 2014, Malagu et al. developed an array of chemoresistive gas sensors based on screen-printed metal oxide semiconducting films to discriminate biomarkers of colorectal cancer with high selectivity, including 1-iodononane and benzene, from those of interference in the gut, such as methane and nitric oxide [80]. The array of sensors was obtained by combining different materials. The best working temperature was determined for each sensor and the responses of different films to the target VOCs were analyzed in a background of realistic concentrations of $\mathrm{CH}_{4}, \mathrm{NO}$ and $\mathrm{H}_{2}$. The most selective sensors to benzene in dry conditions were $\mathrm{TiTaV}\left(\mathrm{TiO}_{2}, \mathrm{Ta}_{2} \mathrm{O}_{5}\right.$ and vanadium oxides) and STN (mixed tin, titanium and niobium oxides) in a methane background. As in the background of $\mathrm{NO}$, the most selective sensors were ST25 $650\left(\mathrm{SnO}_{2}\right.$ and $\left.25 \% \mathrm{TiO}_{2}\right)$ and STN. The best sensors to detect 1-iodo-nonane in a wet ambient environment 
were ST20 $650\left(\mathrm{SnO}_{2}\right.$ and $\left.20 \% \mathrm{TiO}_{2}\right)$, ST25 650 and ST25 + Au1\% $\left(\mathrm{SnO}_{2}, 25 \% \mathrm{TiO}_{2}\right.$ and $1 \% \mathrm{Au}$ ), and their responses increased with concentration (order of $10^{-2} \mathrm{ppm}$ ). The sensor array serves as the basis to set up an electronic sensor network for target gases in colorectal cancer screening. It was suggested that these metal oxide chemoresistive sensors may represent a non-invasive and potentially inexpensive prescreening method for the diagnosis of colorectal cancer and a substitute for preventive colonoscopy.

\subsection{Nanomaterial Sensors}

Gas sensensing based on semiconducting metal oxide has had a considerable impact to date. However, the requirement of operating at high temperatures often limits the use of this type of detection. For these reasons, Korotcenkov modified some of the structural parameters, such as crystal shape and orientation [75]. Nanostructured materials (nanomaterials) offer several advantages compared with semiconducting metal oxide, including low working temperatures, low power consumption, flexibility, easy and safer operation and good chemical selectivity. The nanomaterials include carbon nanotubes, nanowires, nanoparticles and nanofibers. One of the major characteristics of nanomaterials is their exceedingly high surface area and the surface-to-volume or mass ratios which potentially confer high sensitivities due to the higher interaction between the analytes and the sensing materials. 


\subsubsection{Carbon Nanotubes}

Carbon nanotubes (CNTs) have drawn considerable interest due to their unusual structure, properties (such as electronic, mechanical, thermal and optical properties) and possible applications since they were first discovered by Iijima in 1991 [81]. There are two types of nanotubes: single-walled carbon nanotubes (SWNTs) and multi-walled carbon nanotubes (MWNTs). SWCNTs are cylinders consisting of a one-atom-thick layer of graphite with a diameter of several nanometers, and length in the order of micrometers. MWNTs have multiple rolled layers (concentric tubes) of graphene. In 2000, Kong et al. first reported chemical sensors based on SWNTs as sensing materials [82]. The device was fabricated by controlled chemical vapor deposition (CVD) growth of individual SWNTs from patterned catalyst islands on $\mathrm{SiO}_{2} / \mathrm{Si}$ substrates. Figure 1.9 shows the conductance versus time curves of the transistor upon exposure to $\mathrm{NO}_{2}$ and $\mathrm{NH}_{3}$ at room temperature. The conductance of the SWNT sample was observed to sharply increase by about three orders of magnitude on exposure to $200 \mathrm{ppm}$ of $\mathrm{NO}_{2}$ (Figure $9 \mathrm{~A}$ ). The response time (time duration for resistance change by one order of magnitude) was in the range of 2 to $10 \mathrm{~s}$ and the sensitivity (the ratio between resistance after and before gas exposure) was 100 to 1000 . The conductance of the device decreased about 100fold when the SWNT sample was exposed to a $1 \% \mathrm{NH}_{3}$ flow after recovery from $\mathrm{NO}_{2}$ detection (Figure 9B). The response time was about 1 to $2 \mathrm{~min}$, and the sensitivity was about 10 to 100 . 

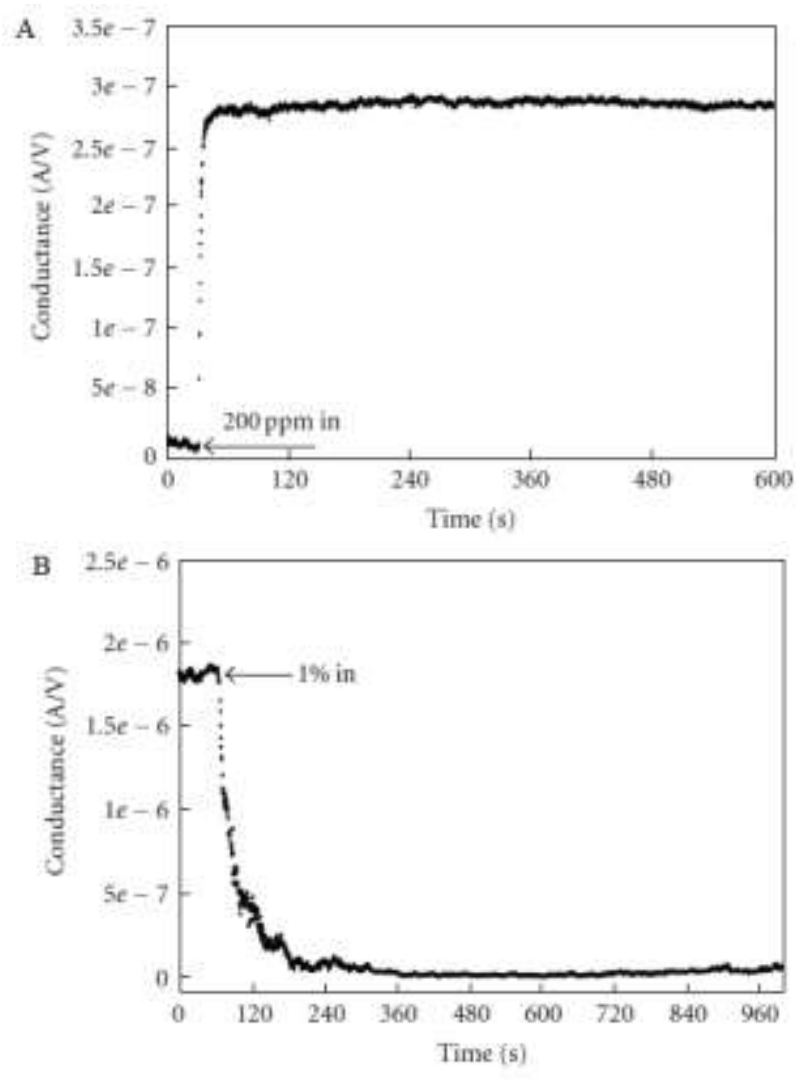

Figure 1.9 Conductance change of SWNT sensor when exposed to (A) $200 \mathrm{ppm} \mathrm{NO}$, (B) $1 \% \mathrm{NH}_{3}$ gases (Reprinted from [82]: J. Kong, et al, 2000)

Compared with SWNTs, the mechanism of MWNTs film sensing VOCs is more complicated due to the multilayer tube structure. However, they also show high sensitivity to specific gases experimentally $[83,84]$. Vertically aligned MWNTs film was synthesized by Huang et al. by thermal CVD and the sensitivity to $\mathrm{N}_{2}$ was tested [85]. The MWNTs showed p-type semiconducting property and the source and drain resistance increased when exposed to $\mathrm{N}_{2}$. Figure 1.10 shows the electrical resistance between source and drain at $10 \mathrm{~V}$ bias voltage without any gate voltage under various $\mathrm{N}_{2}$ filling pressure from $50 \mathrm{~m}$ Torr to 500 Torr. The resistance increased when the 
sensor was exposed to $\mathrm{N}_{2}$ due to $\mathrm{N}_{2}$ acting as an electron donor to CNTs and decreasing the concentration of conducting holes on CNTs. Also, it was obvious that high $\mathrm{N}_{2}$ pressure made the electrical resistance higher.

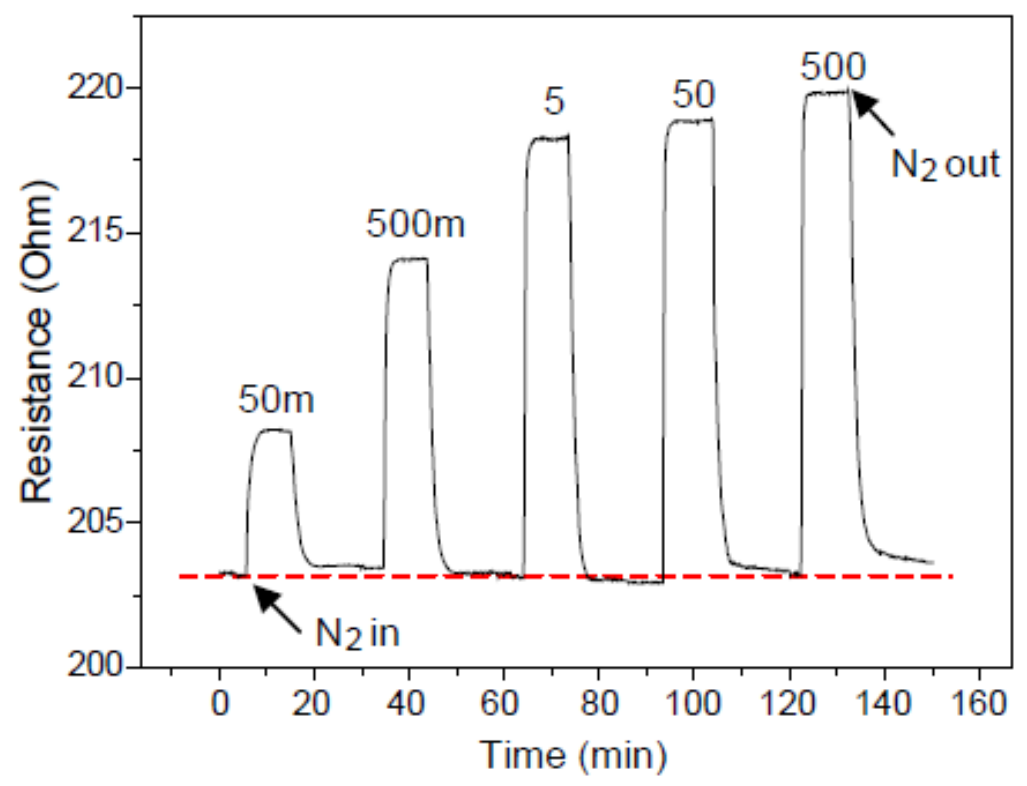

Figure 1.10 The electrical resistance between source and drain at $10 \mathrm{~V}$ bias voltage without any gate voltage under various $\mathrm{N}_{2}$ filling pressure from $50 \mathrm{~m}$ Torr to 500 Torr. (Reprinted from [85]: C.S. Huang, et al. 2005)

\subsubsection{Metal Nanoparticles}

Metal nanoparticles are dispersed on the surface of a substrate to increase the ratio of surface area to volume, and favor the adsorption of the gases. The electronic properties or the properties of the substrate change when the nanoparticles are in contact with the analytes. Several reviews about the synthesis, properties and 
applications of metal nanoparticles are published, including charge transport [86], electrochemistry [87], applications in chemiresistive sensing [68, 88, 89], catalysis, chemical and biological sensing [90], and medicine [91]. Various metals have been employed, such as $\mathrm{Au}, \mathrm{Pt}, \mathrm{Pd}, \mathrm{Ag}$ and alloys of these metals. However, $\mathrm{Au}$ nanoparticles, with organic functional groups, are the most common chemically modified metal nanoparticles used in chemiresistive sensing applications so far. During measurements, VOCs contact and react with tailored organic functional groups, leading to alterations in the connections between the conductive inorganic nanomaterials, or in some cases leading to a charge transfer between the organic functional groups and the inorganic nanomaterials. These alterations cause changes in the measured conductivity $[92,93]$.

In 2005, Han et al. reported nanostructured sensing arrays for the detection of VOCs and nitro-aromatic compounds (NACs) [66]. The nanostructured array elements consist of thin film assemblies of alkanethiolate-monolayer-capped gold nanoparticles that were formed by molecularly mediated assembly using mediators or linkers of different chain lengths and functional groups. Figure 1.11 shows the response characteristics for the sensing responses obtained for three vapors at the five-sensor array system. These sensing elements display linear responses to the concentrations of the vapors, and the response sensitivities vary dramatically as evidenced by the differences in the slopes of the linear relationships. 

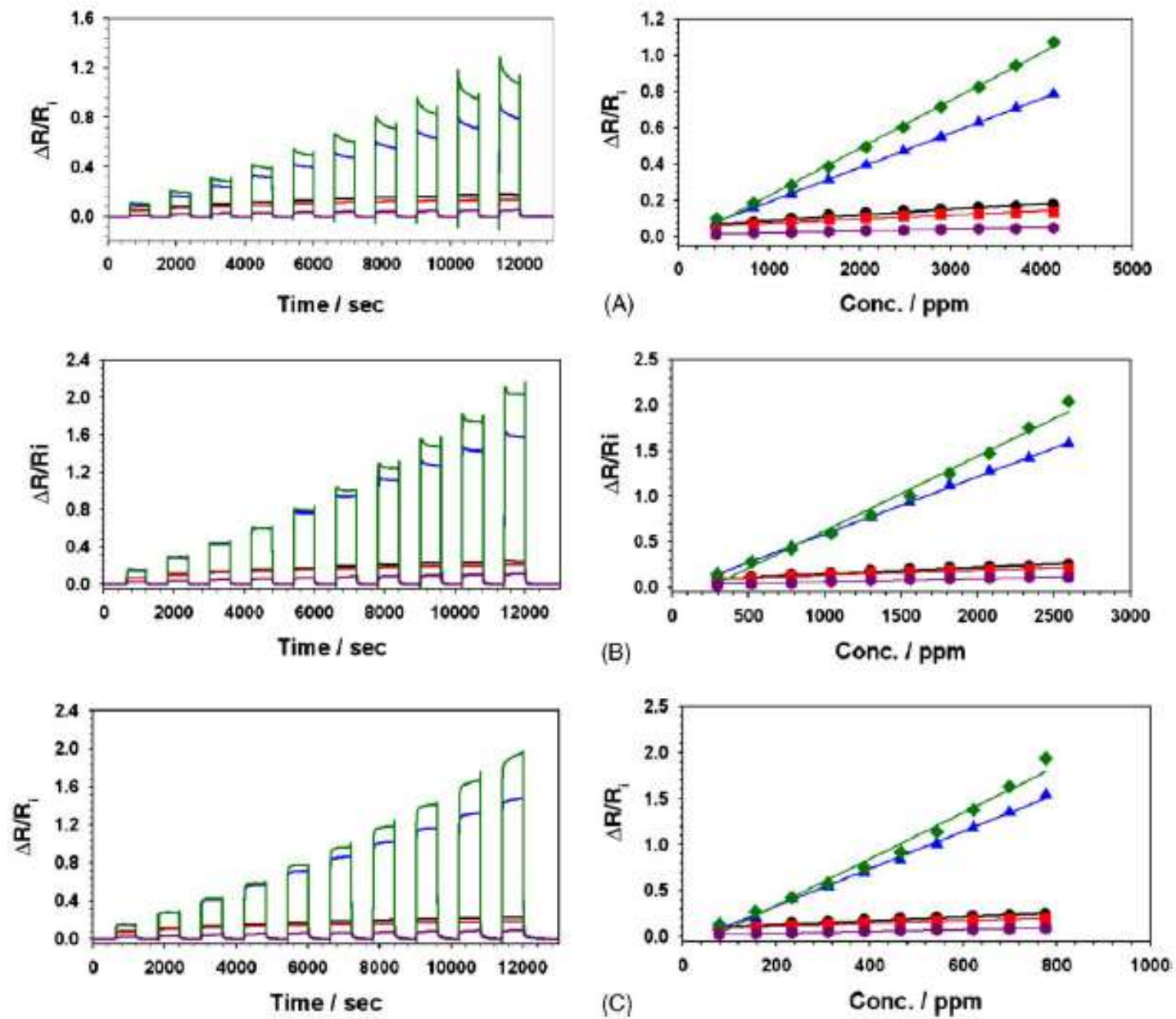

Figure 1.11 Sensor response to $\mathrm{Hx}(\mathrm{A}), \mathrm{Bz}(\mathrm{B})$ and $\mathrm{Tl}(\mathrm{C})$ vapors. Left: response profiles for Hx (413, 826, 1239, 1652, 2065, 2478, 2891, 3304, 3718, and $4131 \mathrm{ppm}$, (A)); Bz (260, 519, 779, 1038, 1298.0, 1558, 1817, 2077, 2336, and $2590 \mathrm{ppm},(\mathrm{B}))$; and $\mathrm{Tl}(78,155,234,310,390,466,546,621,702$, and 776 ppm, (C)). Right: corresponding response sensitivities. Sensor array materials: NDT-Au2-nm (black), PDT-Au2-nm (red), MUA-Au2-nm (blue), MHA-Au2-nm (dark green), MPA-Au2nm (dark pink). (Reprinted from [66]: Han et al. 2005) 
In 2009, Peng et al. fabricated an array of sensors based on functionalized gold nanoparticles. This sensor array can distinguish lung cancer patients from healthy subjects without the need for dehumidification or pre-concentration of the exhaled breath in any way [19]. Figure 1.12 shows the illustration of the diagnosis of lung cancer using breath testing. The results show great promise for fast, easy and cost-effective diagnosis and screening of lung cancer. The developed gold nanoparticle VOC sensor array could provide a simple, portable, inexpensive and noninvasive tool for detection of lung cancer.

a

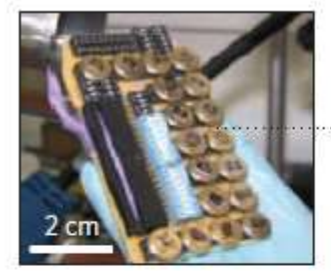

(ii)

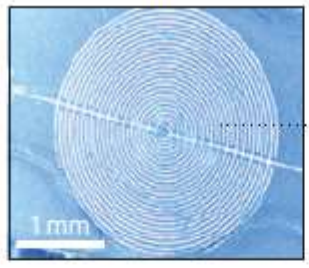

(iii)

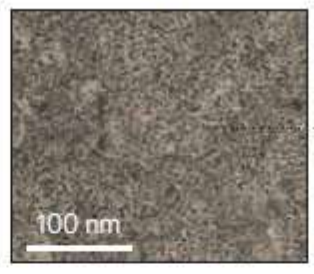

(iv)

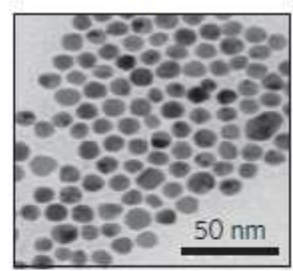

b

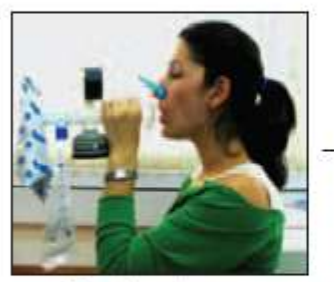

Breath collection

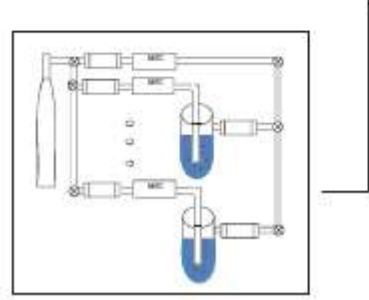

Simulated breath

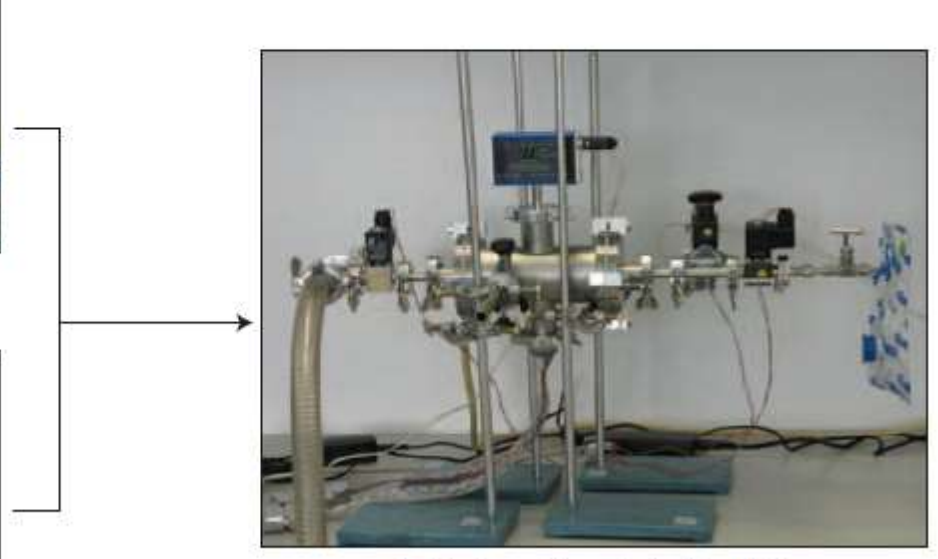

Application of the collected breath to the sensors array

Figure 1.12 Illustration of the diagnosis of lung cancer using breath testing. (a), A photograph of the array of chemiresistors. (b), Testing the exhaled breath collected 
from patients and simulated breath using the array of gold nanoparticle sensors. (Reprinted from [19]: Peng et al., 2009)

\subsubsection{Polymer Nanofibers}

Several recent studies have reported the development of different types of gas sensors in which nanofibers or nanowires are used to detect trace amounts of harmful gases effectively and rapidly [48, 94-98]. In particular, conducting polymer nanofibers have been developed rapidly in various applications including chemical sensors [64, 99-103], metallic materials in electronic devices [104, 105], electromagnetic radiation shielding [106] or interconnects in circuits [107]. The sensing mechanisms of conducting polymers can involve redox reactions, ion adsorption and desorption, volume and weight change, chain conformational changes, or charge transfer and screening. Conducting polymers have an advantage in achieving high sensitivity and selectivity due to their chemical and structural diversity compared to the inorganic counterparts.

Electrospinning is considered a convenient method to fabricate polymer nanofibers with controlled diameters ranging from tens of nanometers to micrometers $[108,109]$. This approach uses an electrostatic field to form and accelerate liquid jets from the tip of a needle. The electrospinning process and a SEM image of electrospun fibers are shown in Figure 1.13 [110]. The apparatus consists of a high voltage power source, a syringe pump and a collector. 

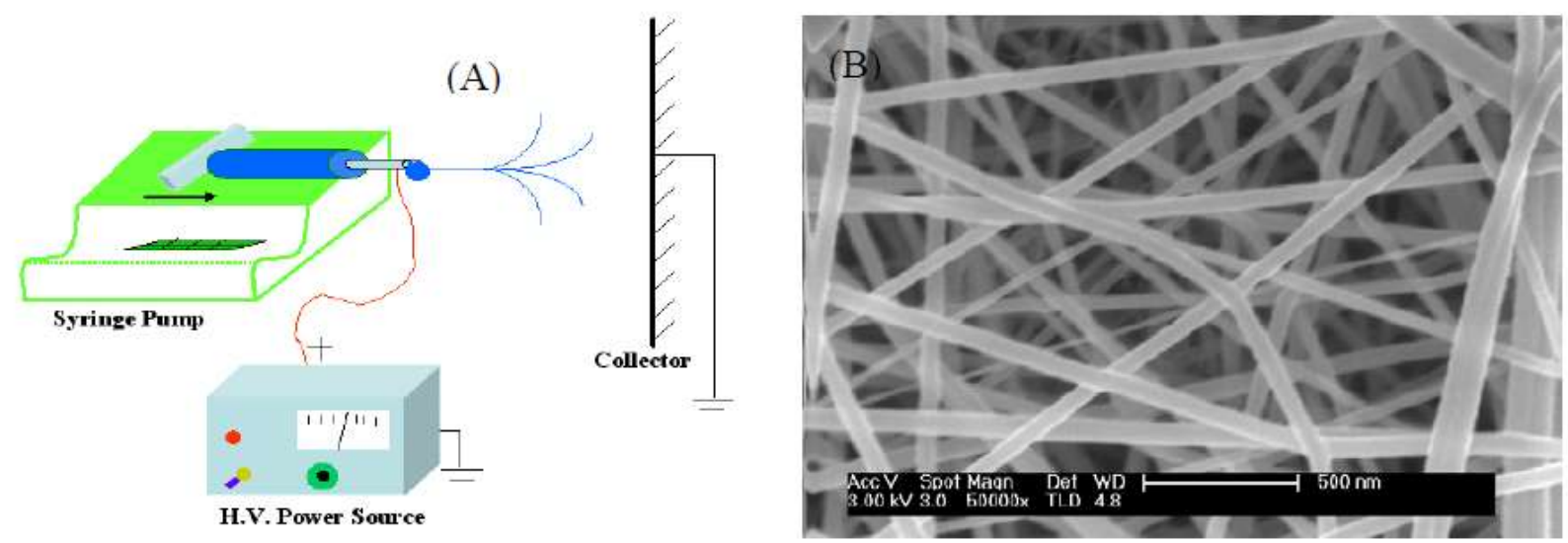

Figure 1.13 (A) A schematic of electrospinning process. (B) A SEM image of typical electrospun fibers. (Reprinted from [110]: Ding, et al.)

Several conducting polymers and their composites have been investigated for practical applications, which are summarized in Table 3. All of them possess high conductivity and good environmental stability. The selective absorption of VOCs can change the volume of the polymer composite, leading to the alteration of resistance between the electrical contacts [111]. Among these polymers, polyaniline (PANI) is a frequently investigated electrically conducting polymer as gas sensing material. For example, Macagnano reported rapid responses and good analyte sensitivity with various blends of PANI and insulating host polymers (polystyrene, polyethylene oxide (PEO) and polyvinylpyrrolidone (PVP)) [112]. PANI-PEO was the most porous layer with good electrical performance and high sensitivity to ammonia, but was the most fragile sensor when encountered by high humidity and temperature. PANI-PVP tended to be a good sensor of $\mathrm{NO}_{2}$, but it was strongly influenced by humidity. PANIPS could be a stable and promising sensor since it is only slightly affected by 
humidity (up to $50 \% \mathrm{RH}$ ). Overall, the conducting polymer sensors are attractive for their low cost, low power consumption, ease of measurement and good performance at room temperature.

Table 3 Representative conducting polymers (Reprinted from Yoon, H.)

\begin{tabular}{l}
\hline \multicolumn{1}{c}{ Name } \\
Polypyrrole (PPy) \\
Polyaniline (PANI) \\
Polythiophene (PTh) \\
$\begin{array}{l}\text { Poly(3,4-ethylenedioxythiophene) } \\
\text { (PEDOT) }\end{array}$
\end{tabular}

\section{Motivation of This Work}

Due to the high demands by the Department of Homeland Security for efficient methods to detect explosives and chemical warfare agents and by the Environmental Protection Agency to monitor air quality for compliance with environmental regulations, rapid on-site detection of trace amounts of VOCs in air becomes an important research area. Rapid detection of trace toxic gases requires amenability to multiple locations, fast analyses and even quantification of the gas species of interest. The detection of trace VOCs is very challenging for traditional 
analytical methods because target VOC concentrations are low and they are mixed with other interfering gases. GC or MS based techniques do not meet the requirements for on-site monitoring of environmental air quality because of the disadvantages of long sample process times, low sampling frequency, limited detection range, and large, unwieldly instruments.

Gas sensors have been used for many applications, such as detecting trace chemical warfare agents, explosive chemicals and monitoring emissions of air pollutants. Metal oxides, particularly p-type and n-type wide band gap semiconductors, such as $\mathrm{ZnO}, \mathrm{SnO}_{2}, \mathrm{TiO}_{2}, \mathrm{WO}_{3}$, and $\mathrm{In}_{2} \mathrm{O}_{3}$, have been used for gas sensing. Target gases either provide or consume electrons from the sensing metal oxides upon adsorption, thus inducing measurable conductivity changes in the metal oxide thin films. Metal oxide based gas sensors have many advantages over other types of gas sensors including rapid response and recovery times, simple sensor structure and low cost. However, it is extremely difficult to use one or two gas sensors to analyze target VOCs in air because of the interference of other trace VOCs. In recent years, microfabricated metal oxide gas sensor arrays, or so-called electronic noses, have shown promise for the analysis of complex VOC mixtures in air and in exhaled breath. However, performance issues, such as the challenges of high power consumption, low sensitivity and poor selectivity of metal oxide gas sensors have prevented microfabricated gas sensor arrays from being widely applied. Thus, there is a critical need to develop real-time, portable, high performance sensors to detect VOCs of interest at ppm thresholds. 
Herein, we propose novel functionalized gold nanoparticle based gas sensor arrays for on-site detection of target VOCs in air and exhaled breath. These sensors have better chemical sensitivity, selectivity and lower power consumption and are inexpensive, portable, easy to use and can obtain data in real time. Therefore, the sensor based VOC detection techniques have huge potential for point-of-care use.

\section{Objectives of This Work}

The overall objective of this thesis work is to develop a sensor array for analysis of VOCs in air and exhaled breath. Selected thiols have been used to modify AuNPs for detection of VOCs in air and exhaled breath. Four specific objectives are described as below:

(1) Design and develop a functional motif to actuate specificity in detection of selected endogenous VOCs.

(2) Synthesize gold nanoparticles with different of thiols. Analyze the sensitivity and selectivity of the sensors between different gold-thiolate nanoparticles for the detection of VOCs in air and human breath and establish a linear regression model of the response with increasing concentrations of VOCs.

(3) Understand the basic mechanism of the conductivity of gold nanoparticle films by exploring the interaction between sensing film and VOCs and the effect of different thiol functional groups.

(4) Design an electronic nose for VOC analysis in air. 


\section{Dissertation Organization}

The dissertation consists of six chapters including present chapter. The following summary presents the scope of each chapter.

In the first chapter, the importance and mechanism of chemiresistors and the application in breath analysis are discussed. Different techniques and materials for detection of volatile organic compounds are reviewed. The motivation and objectives of this work are also stated in this chapter. The organization of this dissertation is outlined.

In the second chapter, a brief introduction to several commercial thiol functionalized gold nanoparticle (AuNP) gas sensors is given. Experimental detail on synthesis of thiol functionalized AuNPs and design and fabrication of interdigitated electrodes (IDE) are described. Thiol structure and AuNP characterization is discussed. The sensor measurement process and sensor response characteristics are explained.

Chapter III introduces a new molecular recognition motif to improve the sensitivity and selectivity of the sensors. The synthesis and characterization of gold nanoparticles with tert-butyl monoalkoxyurea thiol are demonstrated. Furthermore, sensing properties of the designed urea thiol sensor and comparison with commercial thiol sensors, as well as long-term stability of the sensors, are studied. The mechanism of urea-carbonyl hydrogen bonding interaction is proposed. 
Chapter IV demonstrates the application of electronic nose for detection of acetone in diabetes from exhaled breath. This application highlights the ability of the electronic nose to discriminate different levels of acetone concentration in breath samples. In this chapter, the microreactor for determination of initial concentration of acetone in breath is described. Humidity effect on the chemiresistors and breath sample storage time are monitored.

In chapter $\mathrm{V}$, three series of urea thiols with urea-NH-carbonyl interactions are synthesized based on the mechanism proposed in chapter III. To develop an optimal acetone-binding substrate for attachment to gold nanoparticles, panels of monoalkoxyurea thiols, alkyurea thiols, and bis-alkoxyurea thiols that differ principally in a control group $\mathrm{R}$ are prepared. Sensor sensitivity and selectivity toward acetone are compared.

The last chapter consists of a summary of conclusions obtained from the previous study and recommendations for future work. 


\section{CHAPTER II}

\section{THIOL FUNCTIONALIZED GOLD NANOPARTICLE GAS SENSOR FOR VOC DETECTION IN AIR}

\section{Introduction}

Over the past few years, nanoparticle-structured materials for use in novel biochemical and chemical sensors have attracted increasing interest [113-118]. As for thiol functionalized gold nanoparticles, they are composed of roughly spherical Au cores surrounded by organic monolayer shells, which are usually an alkanethiolate, $\omega$-functionalized alkanethiolate, or aromatic thiolate (Figure 2.1) [119]. Recent developments in functionalized nanoparticles have provided a new kind of 'hybrid material' that combines the advantages of organic specificity with the robustness and processability of inorganic materials. They have been used in vapor phase sensing devices that can successfully detect VOCs $[66,120]$ or even diagnose lung cancer from exhaled breath [19], since their structure can be easily adjusted, such as the core size and ligand type. These sensors are fabricated by depositing thin films of ligandcapped gold nanoparticles onto interdigitated electrodes [19]. 


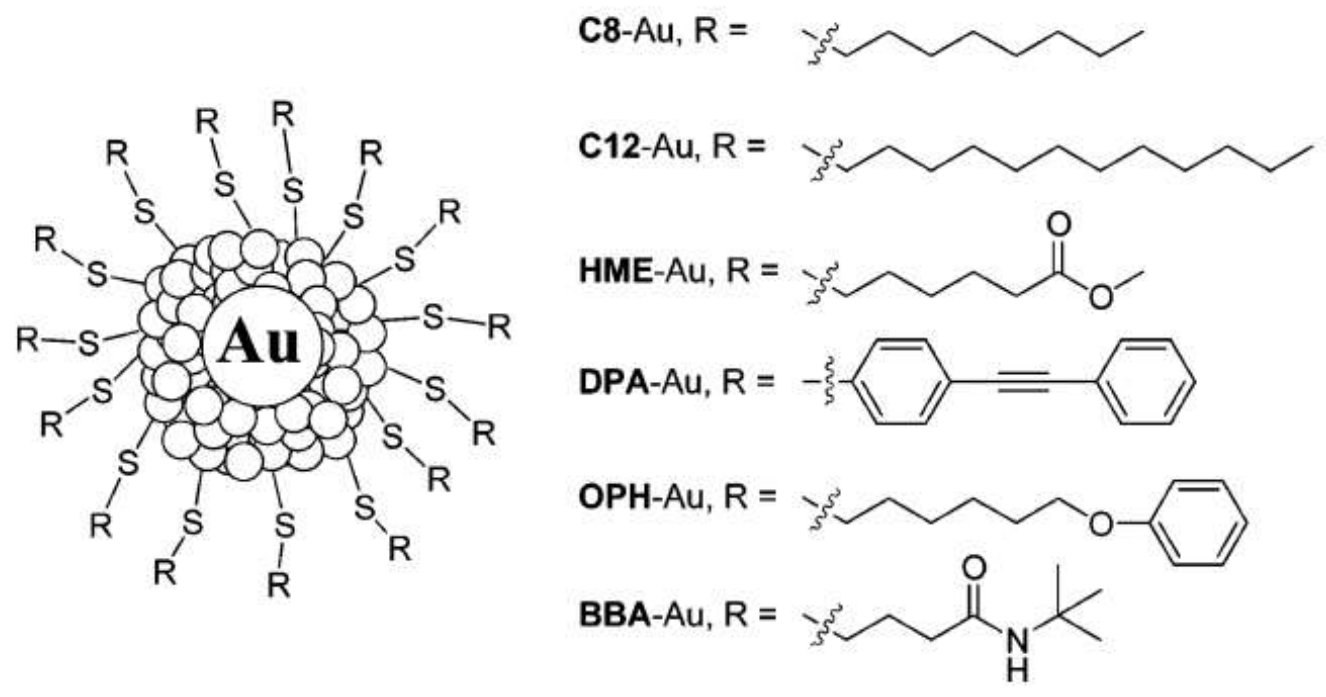

Figure 2.1 Schematic representation of thiol functionalized gold nanoparticle. (Reprinted from Rowe, M.P. et al.)

A typical sensor consists of a VOC delivery system, different thiol functionalized AuNP coating material, and a transduction element that converts a chemical or physical interaction between the analyte and sensitive coating into a measurable electronic signal. When analyte molecules are in the vapor phase, the VOC is transported to the sensor and detected in air under ambient conditions. The vapor phase analytes partition into the sensitive film and a direct change in the resistance of the film provides the signal for quantifying the vapor concentration of the analyte. The concentrations of the analyte in the sensitive film $\left(\mathrm{C}_{\mathrm{f}}\right)$ and the concentration of the analyte in the vapor phase $\left(\mathrm{C}_{\mathrm{v}}\right)$ are related by the thermodynamic partition coefficient $\left(\mathrm{K}_{\mathrm{d}}\right)$ as shown in the following equation $[68,118]$ :

$\mathrm{K}_{\mathrm{d}}=\mathrm{C}_{\mathrm{f}} / \mathrm{C}_{\mathrm{v}}$ 
$K_{d}$ depends on the relative affinity of the vapor phase molecules for the film versus the vapor phase. The sensor response $\left(\mathrm{R}_{\mathrm{s}}\right)$ is usually assumed to be directly related to $\mathrm{C}_{\mathrm{f}}$, which is directly related to $\mathrm{K}_{\mathrm{d}} \mathrm{C}_{\mathrm{v}}$ as follows:

$\mathrm{R}_{\mathrm{s}}=\mathrm{f}\left(\mathrm{K}_{\mathrm{d}} \mathrm{C}_{\mathrm{v}}\right)$

Gold is the preferred choice of metal because of its chemical inertness. Ibanez and Zamborini studied the chemiresistive sensing properties of $\mathrm{Au}$ and AuAg alloy NPs [121]. Silver NP films did not exhibit an appreciable response to any VOCs, although the films were conductive. This could be due to the higher propensity for $\mathrm{Ag}$ to air oxidize compared to $\mathrm{Au}$. In the process of sensor coating, gold nanoparticles provide electrical conductivity to the material.

In this Chapter, we describe the sensor response of AuNPs with different chain lengths and functional groups to acetone, ethanol, benzene and ethylbenzene vapor. We synthesized the AuNPs using a two-phase reduction approach. We designed and fabricated the interdigitated electrode in the cleanroom lab at the University of Louisville. 


\section{Materials and Methods}

\subsection{Chemicals}

Hydrogen tetrachloroaurate $\left(\mathrm{HAuCl}_{4}\right)$, 1-dodecanethiol, 1-octadecanethiol, 4methoxy- $\alpha$-toluenethiol, 11-mercaptoundecanoic acid, tetraoctylammonium bromide (TOAB) and all organic solvents (e.g., ethanol 200 proof) were obtained from Sigma Aldrich. Sodium borohydride was purchased from Fluka. 1-tetradecanethiol and 1hexadecanethiol were purchased from Alfa Aesar. Tedlar bags were purchased from Supelco (Bellefonte, PA). Cylinder dry air was obtained from Welders Supply Co. of Louisville. Deionized water was used throughout the experiment.

\subsection{Synthesis of thiol functionalized gold nanoparticles (AuNPs)}

AuNPs were synthesized using a two-phase reduction approach as reported by Brust et al. [122]. This method delivers particles with diameters in the range 1-3 nm. The following solutions were prepared: $\mathrm{HAuCl}_{4}(0.05 \mathrm{~g})$ was dissolved in deionized water $(4 \mathrm{~mL})$; TOAB $(0.08 \mathrm{~g})$ was dissolved in toluene $(20 \mathrm{~mL})$. The aqueous $\mathrm{HAuCl}_{4}$ solution was added to the toluene TOAB solution with vigorous stirring until all the tetrachloroaurate transferred into the organic layer. A 1:1 thiol: Au molar ratio of thiols then was added in separate experiments to the toluene phase. An aqueous solution of $\mathrm{NaBH}_{4}$, freshly prepared by dissolving $\mathrm{NaBH}_{4}(0.056 \mathrm{~g})$ in deionized water (4 mL), was slowly added with vigorously stirring to the toluene mixture. A 
rapid color change occurred as the $\mathrm{NaBH}_{4}$ solution was added. After stirring for at least $3 \mathrm{~h}$ at room temperature, the organic phase was separated and the AuNPs were precipitated by dropwise addition of the organic phase into ethanol $(400 \mathrm{~mL})$ with rapid stirring. After settling overnight at room temperature, the clear supernatant was decanted and the settled AuNPs were filtered and washed with ethanol. The AuNPs then were dissolved in toluene $(10 \mathrm{~mL})$ and precipitated again by dropwise addition into ethanol (200 mL). After standing overnight at room temperature, the suspension was centrifuged and the collected particles were washed with ethanol and dried at $40{ }^{\circ} \mathrm{C}$ in an oven to yield AuNPs ( $c a .30 \mathrm{mg}$ ) suitable for sensor formation.

\subsection{Interdigitated electrodes (IDE) fabrication and AuNP film deposition}

The interdigitated electrodes (IDE) were designed using the Computer Aided Design (CAD) program L-Edit. L-Edit allows for the design of photomasks for use with photolithography. All IDEs were designed to fit into a $400 \mu \mathrm{m}$ x $400 \mu \mathrm{m}$ area with metal lines $20 \mu \mathrm{m}$ wide and $3000 \mu \mathrm{m}$ long connected to $3 \mathrm{~mm} \times 3 \mathrm{~mm}$ metal contact pads. Similar IDEs were placed in an arrangement to fit onto a standard 4inch silicon wafer. The IDEs were fabricated utilizing the microfabrication technique known as the lift-off process. The lift-off process starts with a sacrificial layer of photoresist coated on substrate. After photoresist is patterned by photolithography, a metal layer is deposited. Finally, the sacrificial layer is removed by dissolving in a solvent. In this way, IDEs and contact pads remain on the wafer. 
Figure 2.2 depicts the process flow diagram of the lift off process used to fabricate the IDEs. A standard 4-inch diameter silicon (Si) wafer (step a) with a thin insulating layer of silicon dioxide $\left(\mathrm{SiO}_{2}\right)(\sim 1 \mu \mathrm{m})$ was coated with LOR-3A, a liftoff promoting resist, and then coated with Shipley 1813 positive photoresist using spin casting (step b). At the recommended spinner settings for LOR-3A and Shipley 1813, an approximately $1.3 \mu \mathrm{m}$-thick layer of photoresist was deposited on the wafer. The wafer was then placed into a Suss MA6/BA6 Mask Aligner and exposed to ultra violet (U.V.) light for 9 seconds, using the dark field mask in Figure 2.2 (step c). The UV light caused the positive photoresist to sinter in the areas exposed. The wafer was then placed into a bath of MF319, a photoresist development solution, for about 30 seconds. The wafer was inspected for the complete development of the exposed photoresist to ensure that IDE features would turn out functional. The wafer was then sputtered with platinum (Pt) using a Kurt J. Lesker PVD 75 DC Sputter (step d). To promote the $\mathrm{Pt}$ adhesion to the $\mathrm{SiO}_{2}$ layer, a thin layer of chromium (Cr) was deposited prior to sputtering the Pt. The $\mathrm{Cr}$ was sputtered for 1 minute and the $\mathrm{Pt}$ for 4 minutes, which results in a $163 \mathrm{~nm}$-thick $\mathrm{Pt} / \mathrm{Cr}$ layer. The wafer was then placed in to an $N$-methylpyrrolidin (NMP) bath with agitation for 24 hours to complete the lift off process (step e). 
a

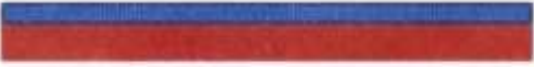

Spin on Photoresist

b

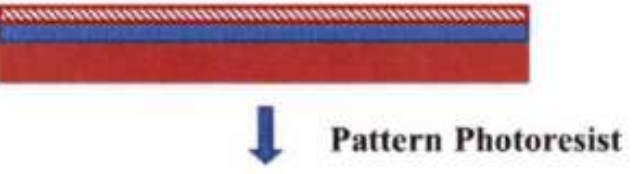

c

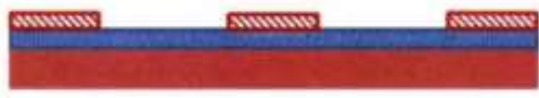

Sputter Platinum

d

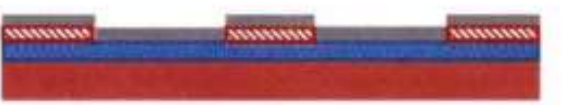

$\downarrow$ Lift off

e

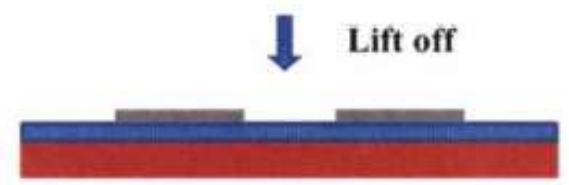

Figure 2.2 IDE fabrication process flow diagram

To complete the IDE fabrication process, the wafer was then diced into $1 \mathrm{~cm}$ by $1 \mathrm{~cm}$ IDE chips. The individual IDEs were then inspected for completeness of the lift off process using a ZEISS Axioskop Optical Microscope. Figure 2.3 displays the microscopic image of gold nanoparticles on the completed IDE. 


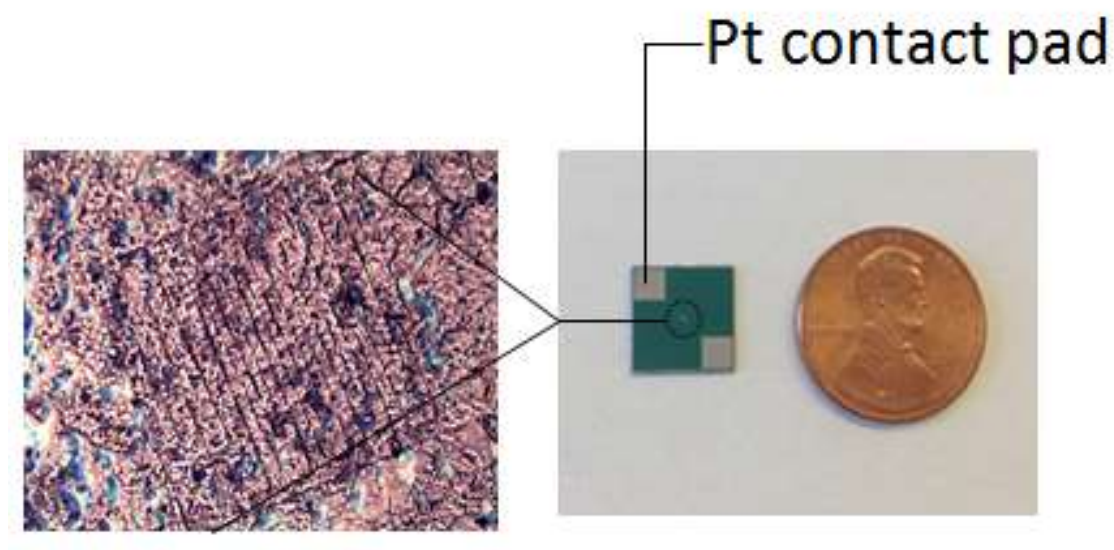

Figure 2.3 SEM micrograph of the gold nanoparticles on interdigital electrodes and optical picture of the sensor device.

The thiol-coated AuNPs in toluene (0.2\%) were dispersed by sonication at room temperature for $20 \mathrm{~min}$ and then cast onto the IDE area by dropwise addition. The toluene was evaporated at room temperature to leave a flat, roughly circular film of gold nanoparticles. The AuNP-coated sensors then were stabilized overnight at $40{ }^{\circ} \mathrm{C}$ in a vacuum oven. AuNP sensors functionalized with 1-dodecanethiol (DDT), 1-hexadecanethiol (HDT), 1-octadecanethiol (ODT), 4-methoxy- $\alpha$-toluenethiol (MTT), and 11-mercaptoundecanoic acid (MUA) were prepared and tested (Table 4). 
Table 4 Thiols used to functionalize the AuNPs chemiresistors

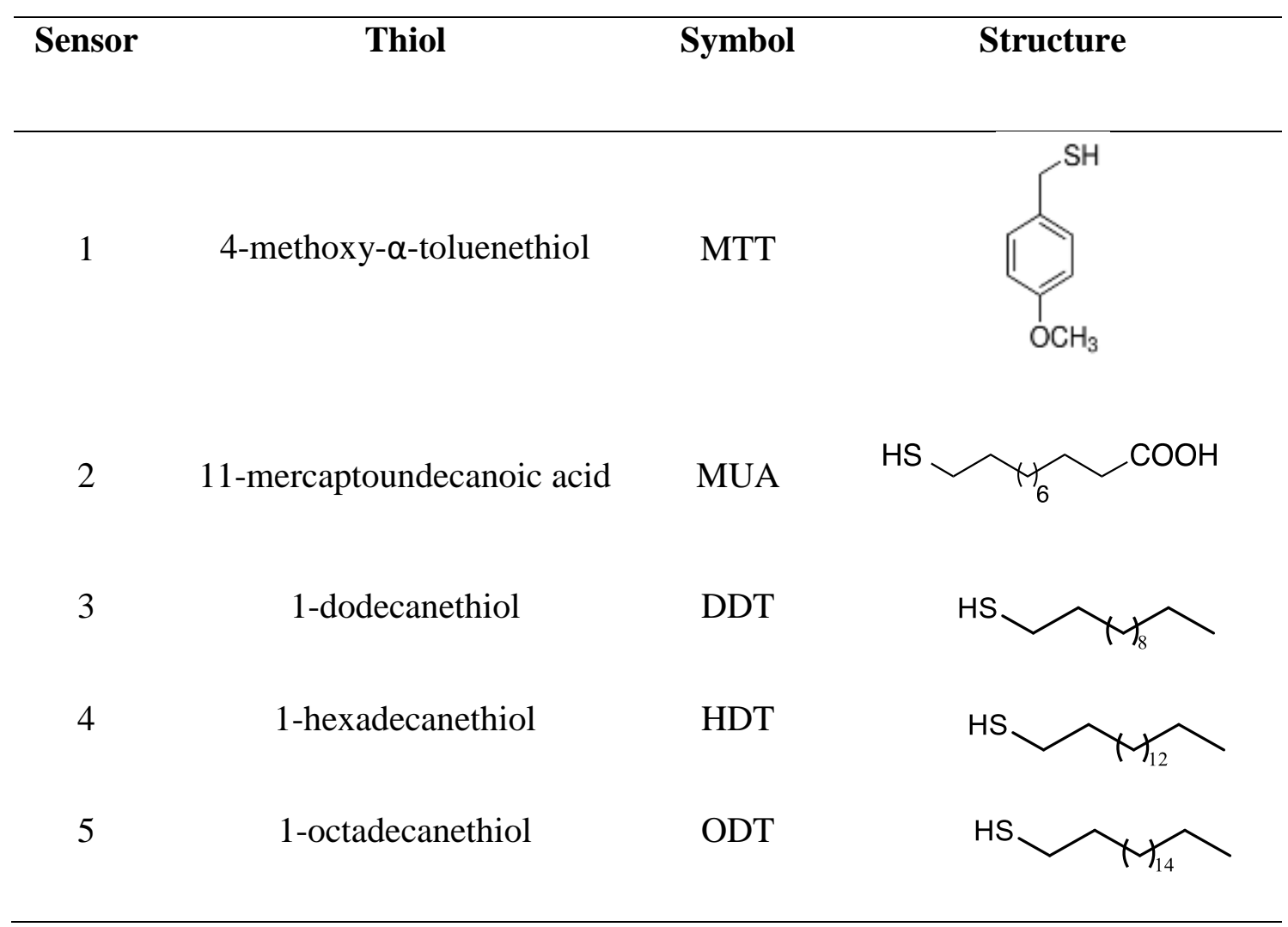

\subsection{Sensor Measurements}

Gas sensors based on AuNPs individually functionalized with the thiol panel of Table 4 were placed inside a homemade stainless steel test chamber. The chamber was first evacuated and then a pulse of a VOC in air was introduced from a sample bag attached to the test chamber. After a given time, the chamber was evacuated again. The VOC samples were prepared using Tedlar bags that had been flushed with dry air three times. An appropriate amount of VOC, for example $3 \mu \mathrm{L}$ of acetone, was injected into a Tedlar bag containing $1 \mathrm{~L}$ dry air. As the acetone vaporizes within 
the bag, a gaseous concentration of $1000 \mathrm{ppm}$ is achieved. Then, $10 \mathrm{~mL}$ of the above gas sample was collected by an air-tight glass syringe and injected into a new $1 \mathrm{~L}$ Tedlar bag followed by a dilution process using $990 \mathrm{~mL}$ dry air, resulting in an acetone concentration of $10 \mathrm{ppm}$. For lower concentrations of analytes, a similar process was carried out again. The acetone concentrations were validated by preconcentration in microreactors as described by Li et al. [123].

The resistance of an individual sensor was monitored at a fixed applied voltage of $5 \mathrm{~V}$ by a Keithley $2400 \mathrm{I}-\mathrm{V}$ meter and recorded as a function of time using the Labview program. The output voltage was set and a pulse current of the sensor was sent to the sensor every second. The resistance was calculated based on Ohm's law. In a typical experiment, sensor response was measured over 5 min under vacuum, followed by gas sample exposure for $5 \mathrm{~min}$, and then again for $5 \mathrm{~min}$ under vacuum. The cycles were repeated two to three times to test reproducibility. Each sensor was examined at different concentrations of acetone, ethanol, ethylbenzene and benzene in air. All experiments were performed at room temperature.

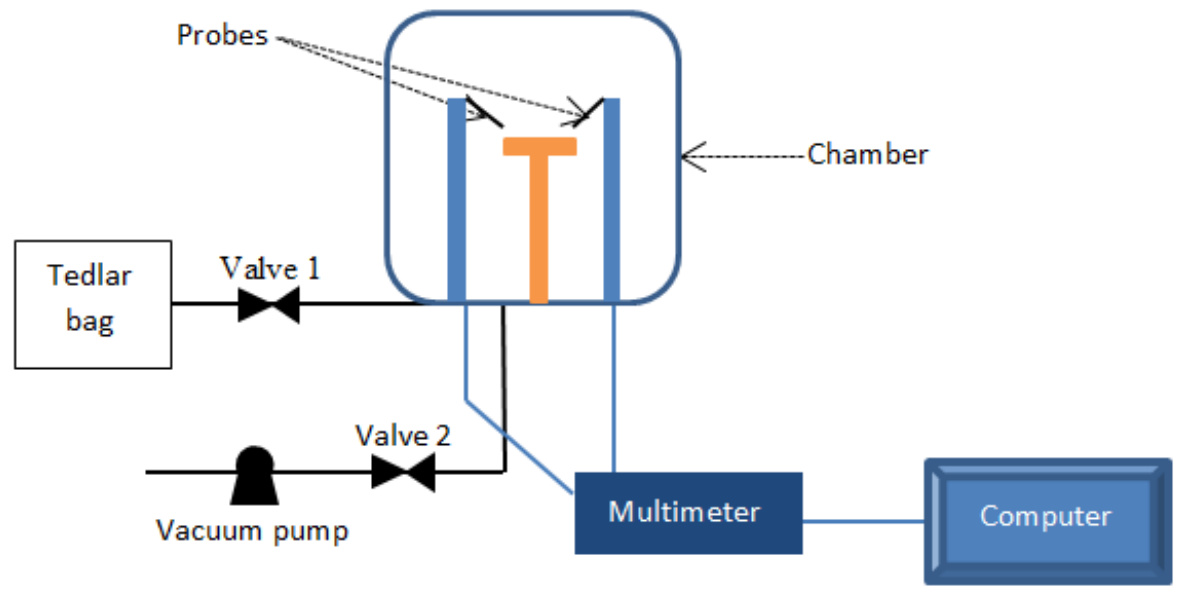

Figure 2.4 Sensor measurement set up diagram 
The sensor response to VOCs in air is defined by the following equation [19]:

$$
\text { Response }=\mathrm{R}_{0} / \mathrm{R}_{\mathrm{gas}^{-}}-1
$$

where $\mathrm{R}_{0}$ and $\mathrm{R}_{\text {gas }}$ are the resistances of the sensor under vacuum and in the presence of the gas to be sensed, respectively. The response and recovery times were defined as the time taken by the sensors to achieve $90 \%$ of the total resistance change in either the adsorption or desorption case.

\section{Material Structure and Characterization}

Transmission Electron Microscopy (TEM) studies were performed using a FEI Tecani F20 transmission electron microscope. A field emission gun (FEG) was used for the electron source and the studies were performed at the accelerating voltage of $200 \mathrm{keV}$. The specimens for examination by TEM analysis were prepared by evaporation of one or two drops of an ethanol solution of the gold nanoparticles on lacey-carbon coated copper grids. Figure 2.5 (a) shows the TEM micrograph of assynthesized DDT functionalized AuNPs. Figure 2.5 (b) represents the histogram of the AuNP size distribution. The size of the AuNPs ranged from 2-4 nm, which is

consistent with thiol monolayer results reported by Brust [122, 124]. AuNPs functionalized with other thiols of similar size range were also observed. 


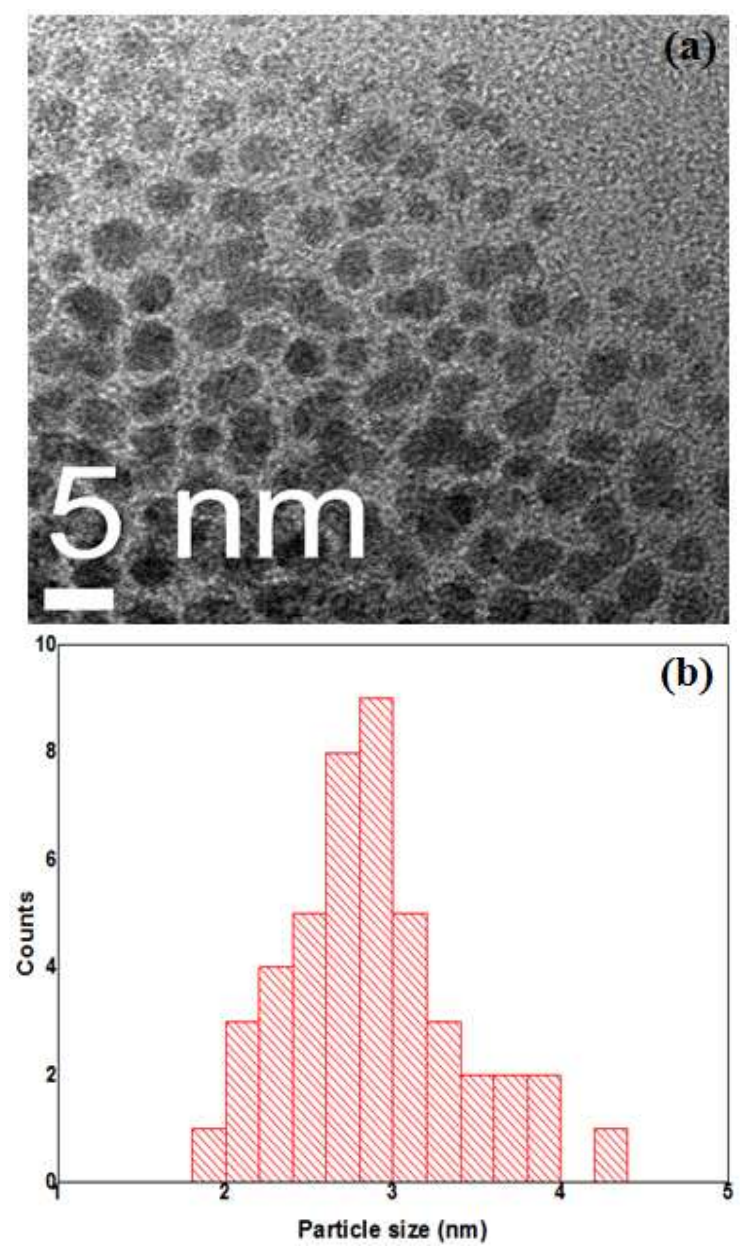

Figure 2.5 (a) TEM micrograph of DDT functionalized AuNP and (b) histogram of AuNP size distribution.

The IR spectrum of the nanoparticles resembles that of 1-dodecanethiol (Figure 2.6) and 4-methoxy- $\alpha$-toluenethiol (Figure 2.7) giving clear evidence that both thiols form part of the composite. The mode assignments are given in the figures. For 1dodecanethiol, all the main peaks are observed in the AuNP. For 4-methoxy- $\alpha$ toluenethiol, the absence of an S-H stretching mode in the spectrum of the particles gives further spectroscopic evidence for the absence of the thiol hydrogen in self- 
assembled monolayer on gold surface, which has been a subject of some discussion in the past [125-127].

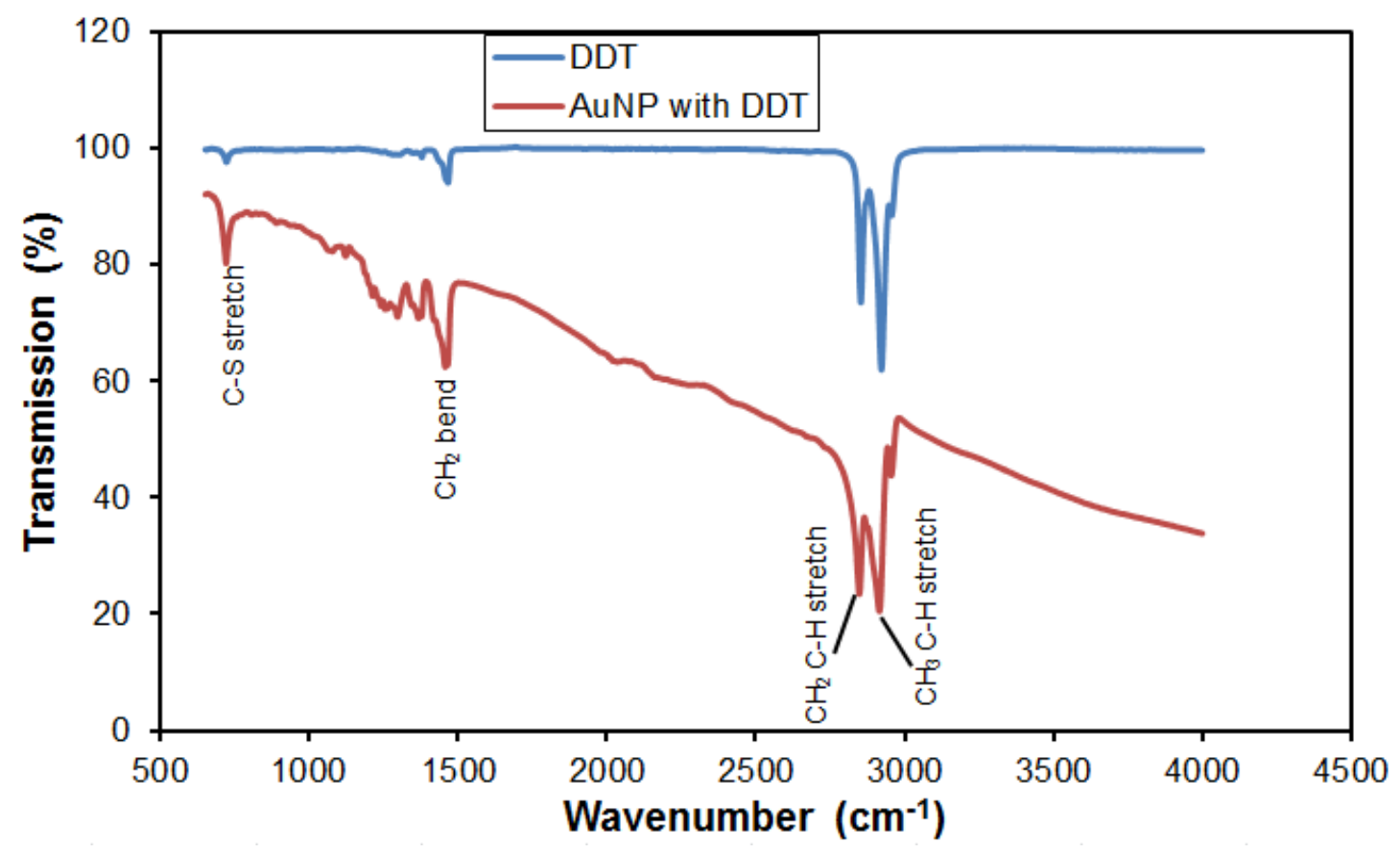

Figure 2.6 IR spectra of DDT and AuNP functionalized with DDT prepared in the present work. 


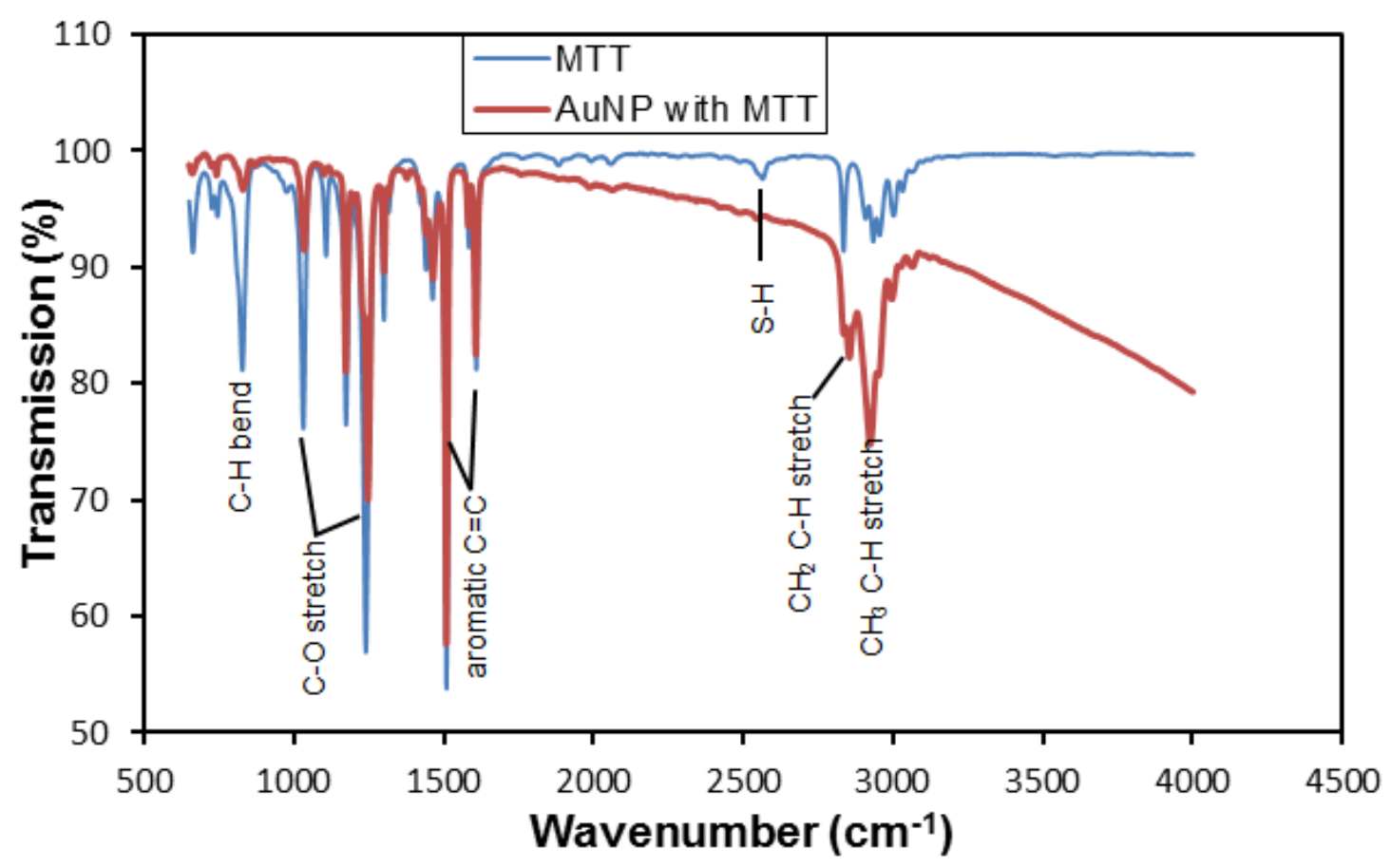

Figure 2.7 IR spectra of DDT and AuNP functionalized with DDT prepared in the present work. The particles were deposited on a disc by evaporation of a drop of an ethanol solution.

TGA is a very useful technique to estimate the thermal stability as well as organic content of organic-inorganic hybrid materials. To understand the extra stabilization offered by thiol moiety present in the AuNPs, TGA analysis was carried out. Figure 2.8 shows the thermogravimetry analysis (TGA) of the AuNP functionalized with (A) 1-dodecanethiol, and (B) 4-methoxy- $\alpha$-toluenethiol. For TGA measurements, 5-30 mg of accurately weighed, extensively dried (vacuum, room temperature) samples were loaded and analyzed under $\mathrm{N}_{2}$ atmosphere in standard aluminum pans. TGA runs were recorded from 30 to $800{ }^{\circ} \mathrm{C}$ at a ramp rate of $10{ }^{\circ} \mathrm{C} / \mathrm{min}$. First, the evaporation of the solvent occurs at around $100{ }^{\circ} \mathrm{C}$. We can see 
almost no weight change for DDT AuNPs around $100^{\circ} \mathrm{C}$ (Figure 2.8A), which is because 1-dodecanethiol is a long alkane chain thiol, so there is little to no water moisture adsorption. Then, the amount of thiol coating on the gold nanoparticles is determined by further heating at $10^{\circ} \mathrm{C} / \mathrm{min}$ through decomposition of the coating. The coating on AuNPs initiates decomposition around $230{ }^{\circ} \mathrm{C}$. The material continues to decompose till $340{ }^{\circ} \mathrm{C}$ with TGA measurements. At $340{ }^{\circ} \mathrm{C}$, the TGA has a residual mass of $82 \%$. Figure 2.8B shows AuNPs functionalized with MTT. There's about 3\% weight loss when the temperature increases from 30 to $100{ }^{\circ} \mathrm{C}$ because of the associated water moisture with the material. After heating to $100{ }^{\circ} \mathrm{C}$, the excess water seems to be driven off and the organic monolayer shells initiate decomposition around $180{ }^{\circ} \mathrm{C}$ and the weight starts to stabilize at $280{ }^{\circ} \mathrm{C}$ with the residual mass of $83 \%$. The loss of organic component can be determined by subtracting the water loss from the total weight loss. It was found that there was $14 \%$ thiol in the as-synthesized MTT functionalized gold nanoparticles. So in $7 \mathrm{mg}$ AuNP, the weight of thiol is 0.98 mg, which leads to $6.4 \mu \mathrm{mol}$ MTT thiol.
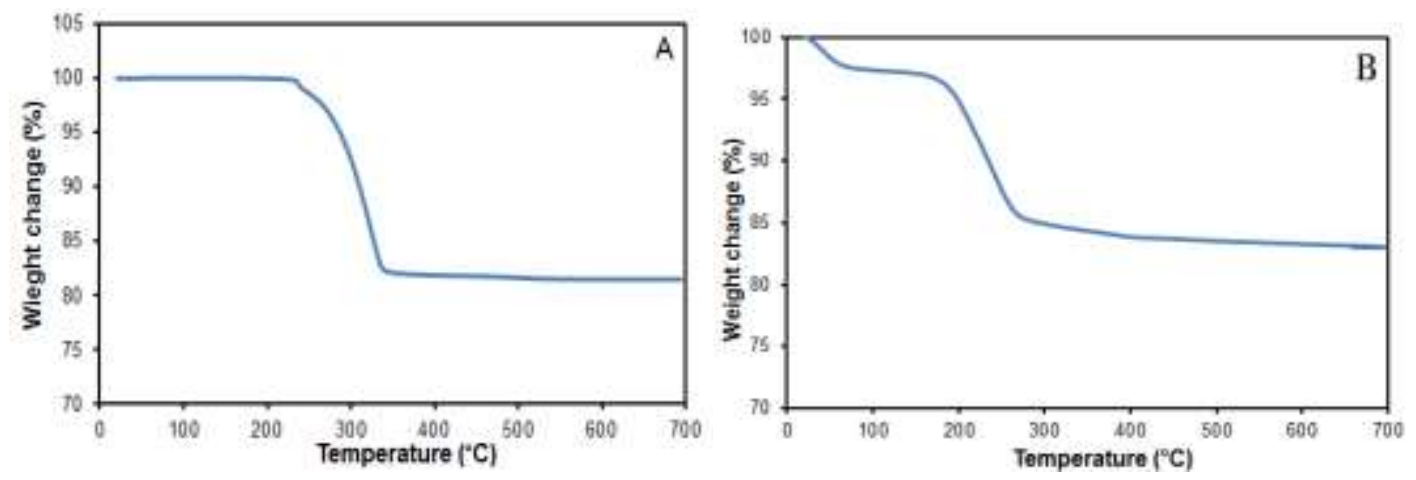

Figure 2.8 TGA of gold nanoparticles functionalized with (A) 1-dodecanethiol, and (B) 4-methoxy- $\alpha$-toluenethiol. 


\section{Sensor Response Characteristics}

In this section, the general response characteristics for the sensor array system with different thiol functionalized AuNP chemiresistors in response to different vapors are described. The response profiles for the five sensing array materials on IDE devices, i.e., MTT-AuNPs, MUA-AuNPs, DDT-AuNPs, HDT-AuNPs, and ODT-AuNPs, to a series of volatile organic vapor analytes were first examined. The VOCs tested included acetone, ethanol, benzene, and ethylbenzene.

The array sensing materials differ from each other in terms of the chemical and physical nature of the mediator molecules, including hydrophobicity, chain length and structure. Because of these differences, the film conductivity properties are different from each other. The differences in the conductivities reflect the difference of a combination of parameters, including core edge-to-edge distance, electron tunneling coefficient, and activation energy of electron transfer, which are important design parameters for the metal nanoparticle-based sensing array materials. 

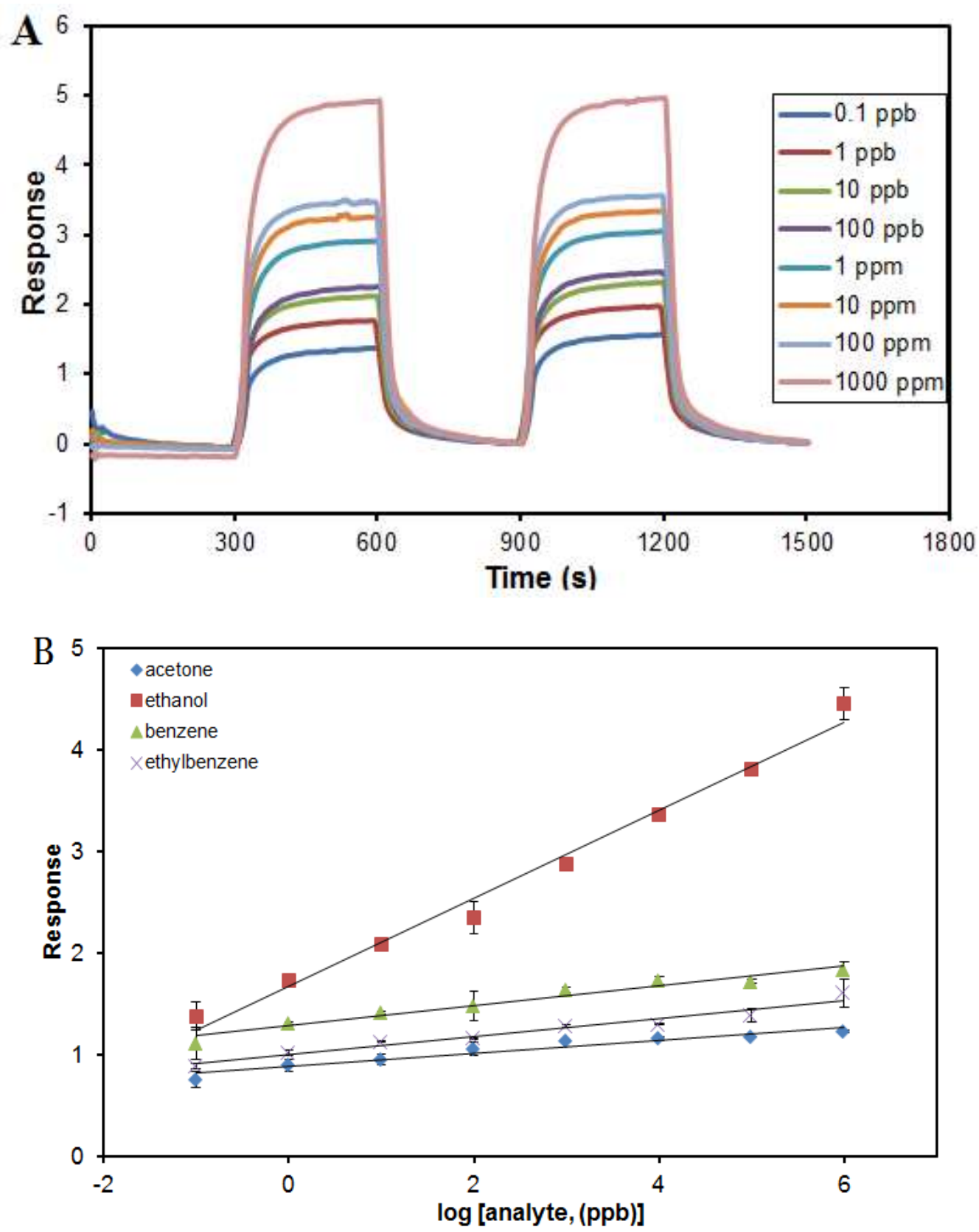

Figure 2.9 (A) Sensor response profile for MTT AuNP film in response to ethanol vapor at the concentrations from $0.1 \mathrm{ppb}$ to $1000 \mathrm{ppm}$, (B) Sensor response versus $\log [$ analyte (ppb) $]$ in detection of acetone, ethanol, benzene and ethylbenzene. The responses are nearly linear. Error bars indicate the variability among three different sensors. Most error bars are smaller than the data symbols in Figure 2.9 (B). 
Figure 2.9(A) shows the response data to ethanol vapor at different concentrations in dry air on MTT AuNP film. The response profile shows that the resistance decreases in the presence of ethanol, which is probably due to the increase in the permittivity of the organic matrix surrounding the metal cores. The resistance returns to baseline value upon chamber evacuation. The response is rapid and reversible. Figure 2.9(B) shows the response characteristics for different vapors at different concentrations for MTT AuNP film. The slope of the linear regression curve serves as a direct measure of the response sensitivity. It indicates that MTT AuNP sensor is most sensitive to ethanol. 

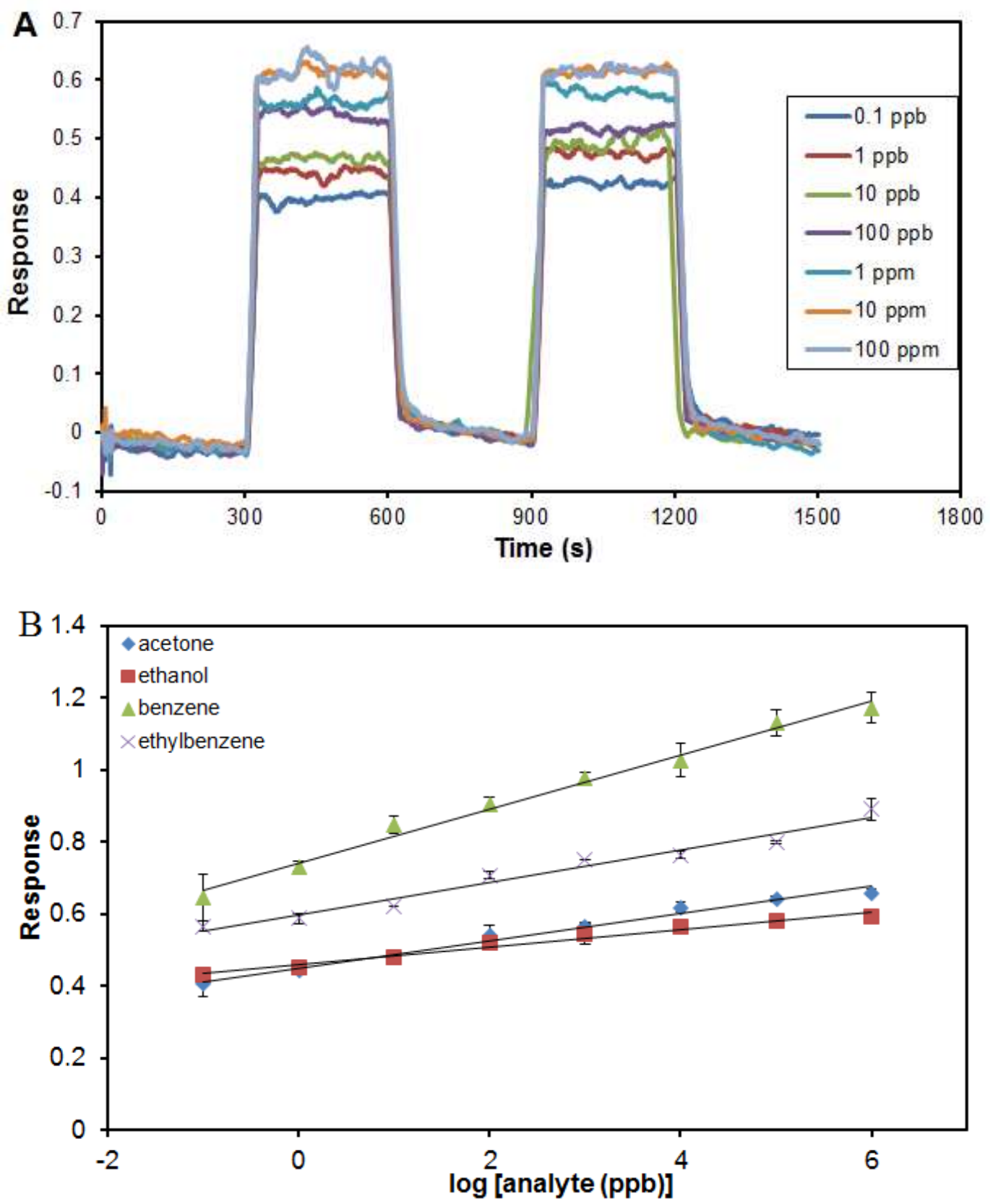

Figure 2.10 (A) Sensor response profile for MUA AuNP film in response to acetone vapor at the concentrations from $0.1 \mathrm{ppb}$ to $100 \mathrm{ppm}$, (B) Sensor response versus $\log [$ analyte $(\mathrm{ppb})]$ in detection of acetone, ethanol, benzene and ethylbenzene. 
We also tested responses of other AuNP films in the array to acetone, ethanol, benzene and ethylbenzene vapor molecules. Figure 2.10 to 2.13 show the response characteristics for the sensing response obtained for these vapors at the sensor array system (sensing film: MUA AuNP, DDT AuNP, HDT AuNP, and ODT AuNP). As can be seen, the calibration curves display fairly linear responses to the log concentrations of vapors. The response of benzene is linear at low concentrations, but it saturates above $100 \mathrm{ppb}$ on DDT AuNP film. Same situation was found for ODT AuNP film to ethylbenzene vapor, which saturates above $1 \mathrm{ppm}$. This occurs because the VOCs have different vapor pressures, so the concentrations are different at different levels of saturation. MUA shows larger response to benzene; however the slopes of the linear regression curve to all the VOCs seem to be similar. 

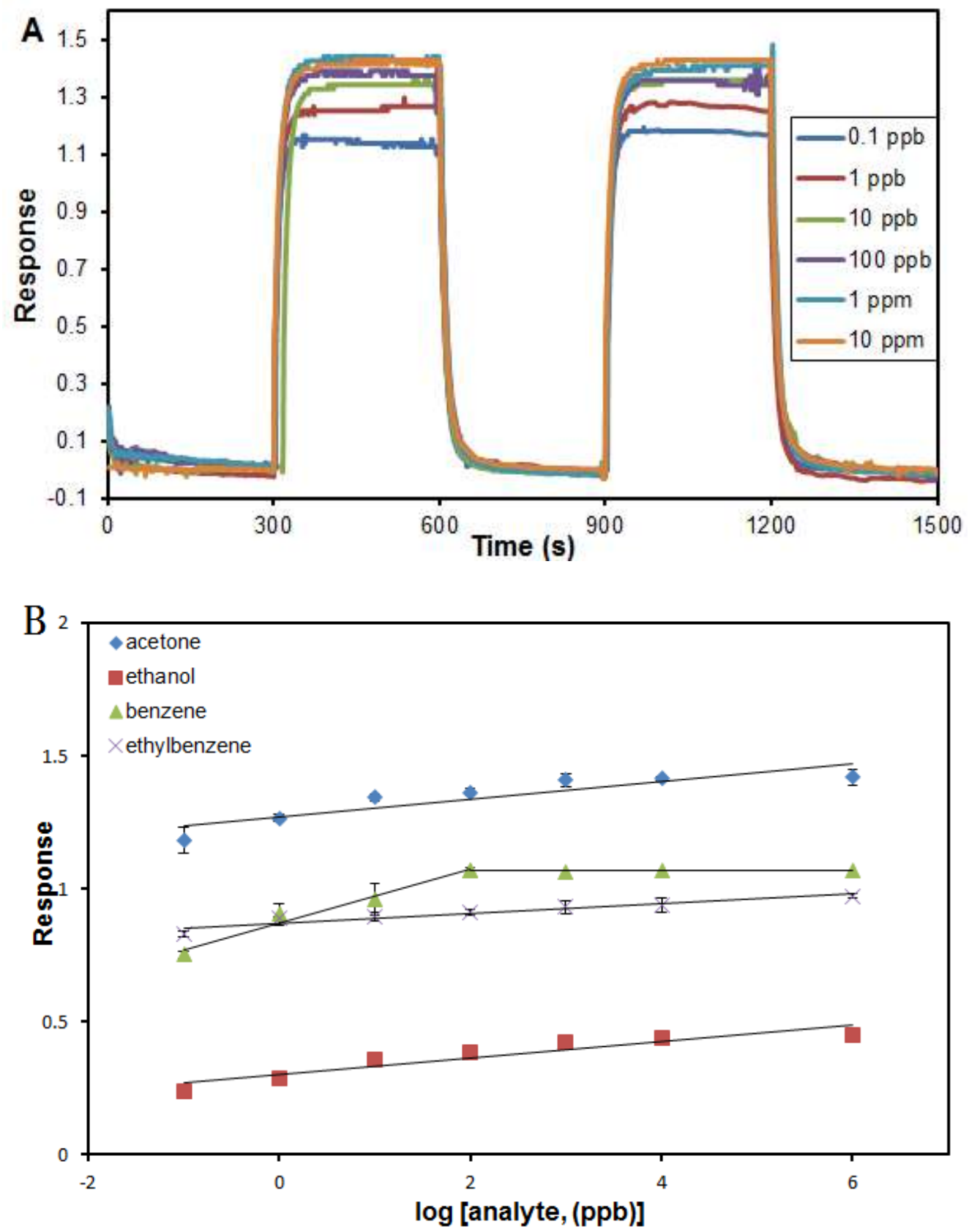

Figure 2.11 (A) Sensor response profile for DDT AuNP film in response to acetone vapor at the concentrations from $0.1 \mathrm{ppb}$ to $10 \mathrm{ppm}$, (B) Sensor response versus $\log [$ analyte $(\mathrm{ppb})]$ in detection of acetone, ethanol, benzene and ethylbenzene. 

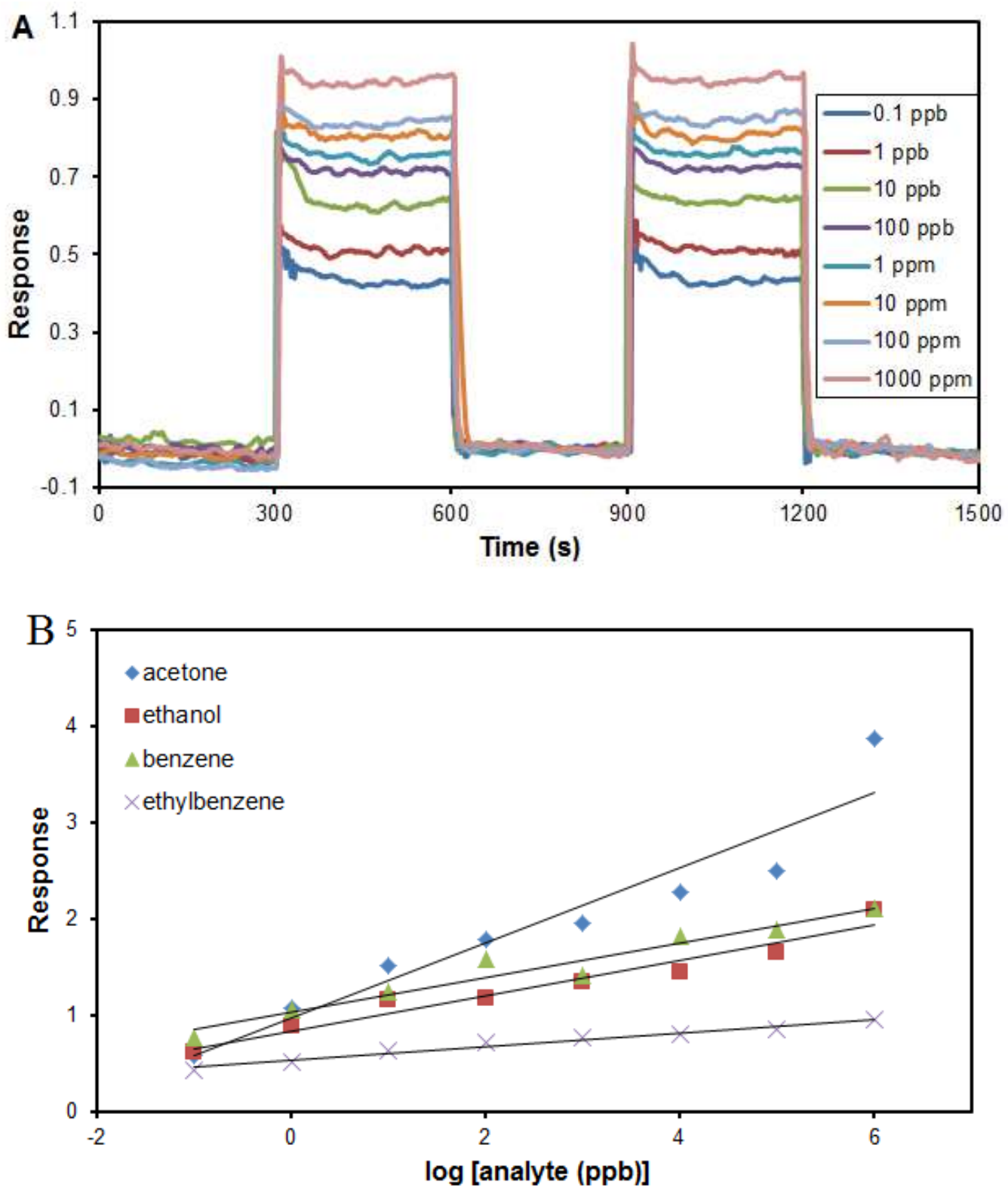

Figure 2.12 (A) Sensor response profile for HDT AuNP film in response to acetone vapor at the concentrations from $0.1 \mathrm{ppb}$ to $1000 \mathrm{ppm}$, (B) Sensor response versus $\log [$ analyte $(\mathrm{ppb})]$ in detection of acetone, ethanol, benzene and ethylbenzene. 

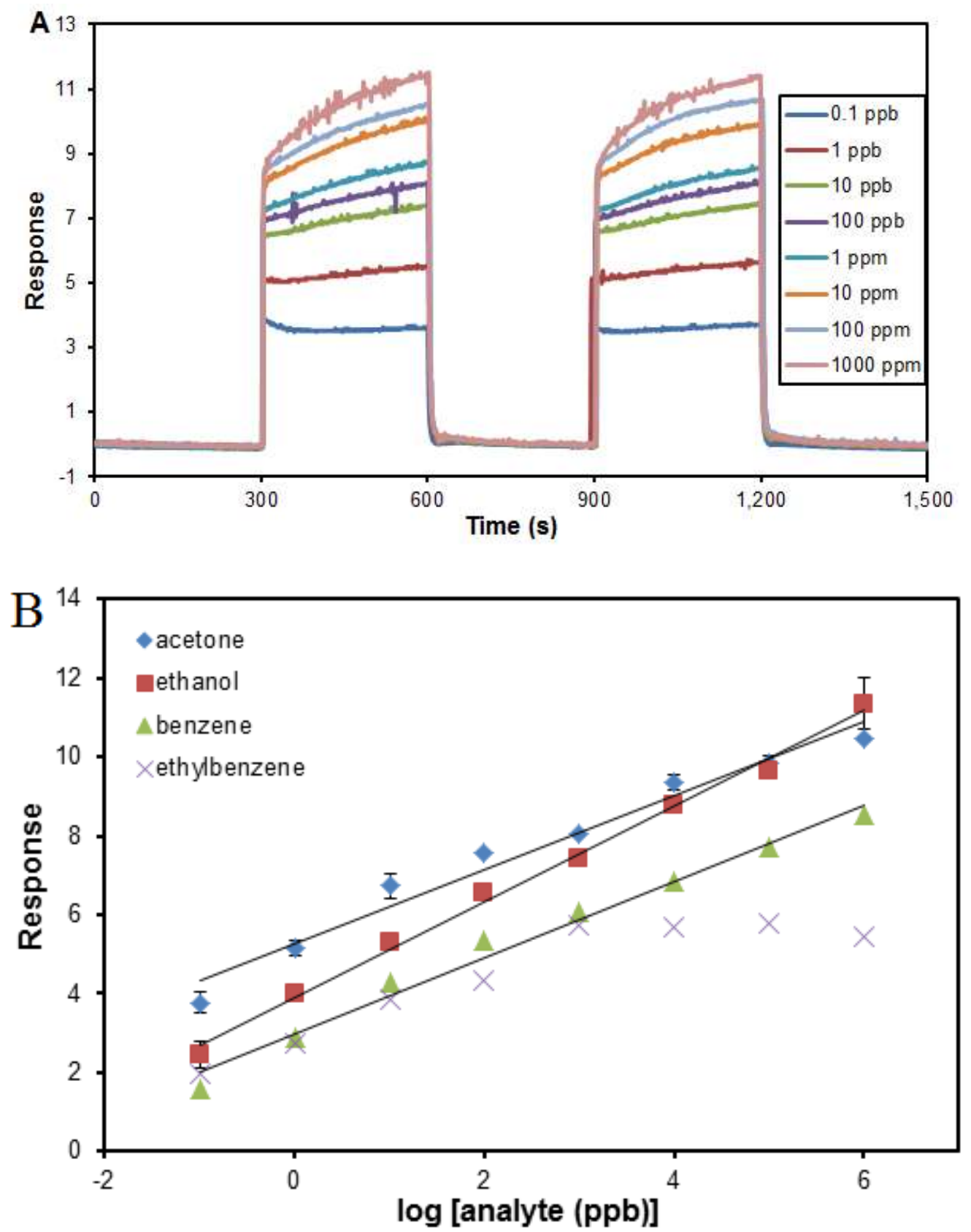

Figure 2.13 (A) Sensor response profile for ODT AuNP film in response to acetone vapor at the concentrations from $0.1 \mathrm{ppb}$ to $1000 \mathrm{ppm}$, (B) Sensor response versus $\log [$ analyte (ppb)] in detection of acetone, ethanol, benzene and ethylbenzene. 
In order to see clearly the interference of different VOCs, here we plot the responses of the five sensors to $0.1 \mathrm{ppm}$ acetone (blue), ethanol (red), benzene (green) and ethylbenzene (purple) (Figure 2.14). At a first glance, all sensors are responsive to every analyte and individual thiols induce different selectivity. The responses to different VOCs increases from DDT $\left(\mathrm{C}_{12} \mathrm{~S}\right)$ to ODT $\left(\mathrm{C}_{18} \mathrm{~S}\right)$ as the alkane chain length increases.

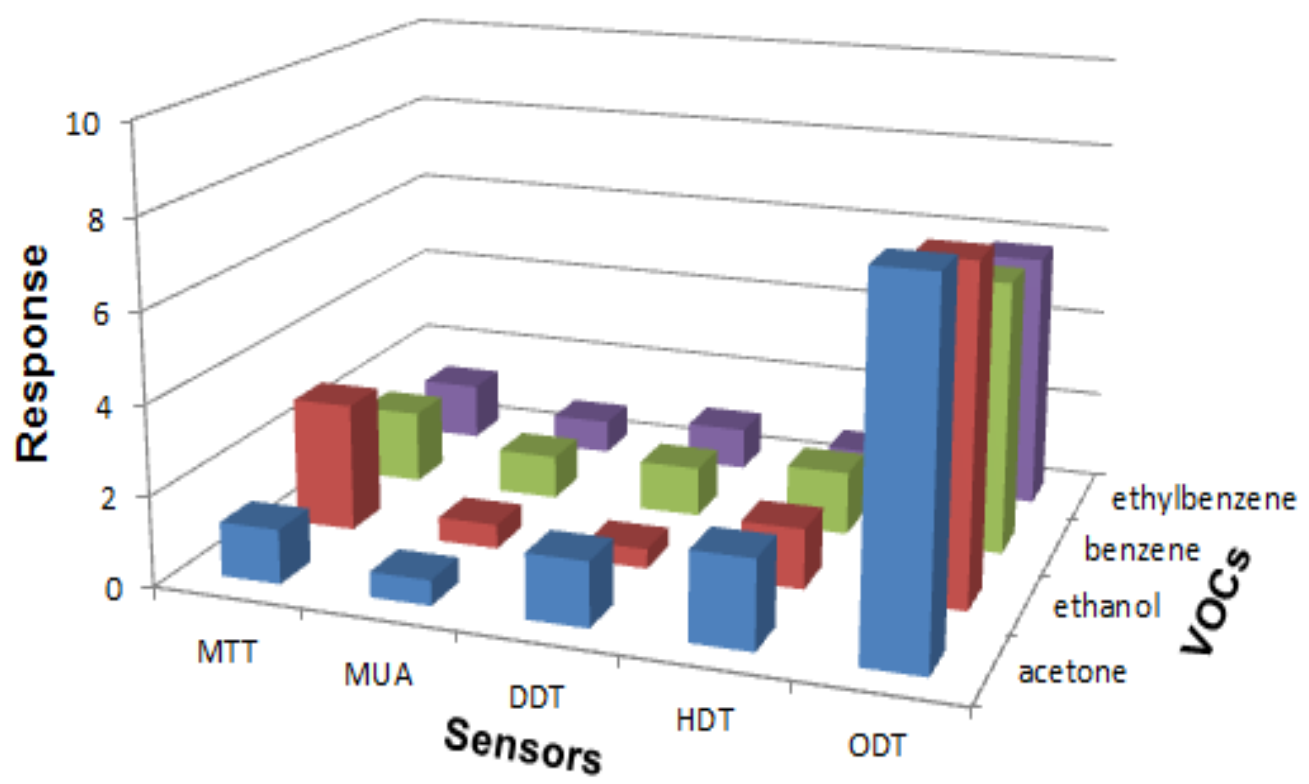

Figure 2.14 Sensor responses to $1 \mathrm{ppm}$ VOCs 
Table 5 Response sensitivities of different vapors on 5 thiol functionalized AuNP sensors (the sensitivity of VOCs for 1-dodecanethiol Au NP sensor was obtained in the linear regime)

\begin{tabular}{cccccc}
\hline VOC & $\mathbf{1}$ & $\mathbf{2}$ & $\mathbf{3}$ & $\mathbf{4}$ & $\mathbf{5}$ \\
\hline Acetone & 0.0533 & 0.0367 & 0.0744 & 0.3877 & 0.9263 \\
Ethanol & 0.4463 & 0.0265 & 0.0496 & 0.1824 & 1.2426 \\
Benzene & 0.0965 & 0.0601 & 0.1615 & 0.1783 & 0.9688 \\
& & & & & \\
Ethylbenzene & 0.106 & 0.045 & 0.021 & 0.0688 & 0.9154 \\
& & & & & \\
\hline
\end{tabular}

Based on the above response data, we further compare the response sensitivities in Table 5. By comparing sensors 3, 4, and 5, we can see the sensor responses and sensitivities are increasing with an increase in the alkyl length surrounding the AuNPs. Films containing the longest alkyl chain length (ODT AuNPs) responded greater to all vapors in the range from $0.1 \mathrm{ppb}$ to $1000 \mathrm{ppm}$. Therefore, while films of AuNPs coated with thiols with a larger number of carbons displayed lower conductivity, they provide more absorption sites for the film to interact with the analyte and swell, and thus improve sensor sensitivity [67]. These films have a larger partition coefficient $\left(\mathrm{K}_{\mathrm{d}}\right)$, which leads to a larger response according to Equation (3) and (4). Even though they show high sensitivity for ODT sensor response, poor 
selectivity is observed for the sensor to VOCs. A further improvement of the sensor selectivity is required in order to differentiate VOCs.

\section{Conclusion}

Here, the capability of molecularly mediated assemblies of gold nanoparticle structured sensing materials for the detection of VOCs is demonstrated. The nanostructured array elements display linear responses to the vapor concentrations. This method can measure the concentrations of VOCs in a wide range from $0.1 \mathrm{ppb}$ to $1000 \mathrm{ppm}$. The 4-methoxy- $\alpha$-toluenethiol sensor is more selective to ethanol, while the ODT sensor is selective to both acetone and ethanol. Also, films with larger number of alkyl chain length in the chemiresistor showed greater responses and sensitivities to VOCs. However, the overall selectivity of these sensors is low, which requires us to seek other ligands to improve the sensor selectivity. 


\section{CHAPTER III}

\section{ACTUATING GOLD NANOPARTICLE CHEMIRESISTOR SENSITIVITY AND SELECTIVITY BY INCORPORATION OF A MOLECULAR RECOGNITION MOTIF}

\section{Introduction}

Poor sensitivity and selectivity are common challenges faced by gas sensing chemiresistors. Devising a functional motif to actuate specificity in detection of select endogenous VOCs requires molecular-level control [128]. The high level of control afforded by H-bonded ensembles in numerous supramolecular structures has inspired us to harness H-bonding to develop a functional sensing device [129, 130]. The unique and parallel-oriented H-bonding networks formed by urea moieties and the complementarity of this functionality with oxygen atoms of oxyanions and other anionic species has given rise to myriads of anion receptors [131, 132], gelators [133, 134], and small molecule catalysts [135]. The seminal report by Kaftory et al. on the role of H-bonding in determining the crystal structures of adducts between acetone and urea derivatives has prompted much effort toward developing urea motifs as receptors for the carbonyl functional group [136]. With this in mind, we set out to 
couple the carbonyl selection properties of urea H-bonded networks with the conductive properties of gold nanoparticles.

Chemiresistors based on metal nanoparticles hold a prominent role in tracing VOCs due to their controllable selectivity, high sensitivity, low detection limits, low out-put impedence, and their ease in integration with other microelectronic systems [92, 137, 138]. Since Wohltjen et al. reported the first ligand-stabilized AuNP chemiresistor for VOC detection [113], numerous researchers have explored AuNPbased sensing platforms in detecting various VOCs [68, 139-143] and under various conditions, including high humidity [144]. However, the fabrication of a chemiresistor that responds selectively to carbonyl substrates and is simultaneously resistant to other chemotropic agents remains to be realized.

To prepare such a chemoselective sensing platform and to provide tailored nanoparticle samples for real-world applications, we examined use of a prototype ligand 1-(tert-butyl)-3-((11-mercapto-undecyl)oxy)urea (1). Thiol was synthesized through acylation of an aminooxy intermediate to afford this unprecedented thiolterminated monoalkoxyurea motif. It then was incorporated onto AuNPs and tested for detecting trace amounts of acetone via reversible non-covalent H-bonding interactions. 


\section{Synthesis of Gold Nanoparticles with Monoalkoxyurea Thiol 1}

\subsection{Materials and Methods}

Reagent grade solvents were used for extractions and for purifications via flash column chromatography. Tetrahydrofuran (THF) was dried by distillation over Na/benzophenone. Dichloromethane (DCM) and dimethylformamide (DMF) were dried by distillation over $\mathrm{CaH}_{2}$.

The progress of reactions was monitored by thin-layer chromatography using silica gel $60 \mathrm{~A}^{\circ} \mathrm{F}-254$ plates. The plates were visualized first by UV illumination and then by staining using a $p$-anisaldehyde stain. Column chromatography was performed using silica gel (230-400 mesh).

11-Bromo-1-undecene, thioacetic acid, and tert-butyl isocyanate were purchased from Sigma-Aldrich. N-Alkoxypthalimide 2 (Figure 3.1) was prepared according to a literature procedure [145]. 1-(tert-Butyl)-3-((11-mercaptoundecyl)oxy)urea(1) was prepared by analogy to a reported method [146].

NMR spectra were obtained using a Varian/Agilent 400-MHz NMR spectrometer equipped with a $5 \mathrm{~mm} \mathrm{z}$-axis gradient AutoX probe operating at the nominal ${ }^{1} \mathrm{H}$ frequency of $399.66 \mathrm{MHz}$ and ${ }^{13} \mathrm{C}$ frequency of $100.49 \mathrm{MHz}$. All spectra are reported in parts per million (ppm) relative to the residual solvent peak in ${ }^{1} \mathrm{H}$ NMR and the deuterated solvent peak in ${ }^{13} \mathrm{C}$ NMR. Mass samples were analyzed on a hybrid linear ion trap (LIT) FT-ICR mass spectrometer (LTQ-FT, Thermo Electron, Bremen, Germany) equipped with a TriVersa NanoMate ion source (Advion 
BioSciences, Ithaca, NY) with an "A' electrospray chip (nozzle inner diameter 5.5 $\mathrm{lm})$. The TriVersa NanoMate was operated in positive ion mode by applying $1.85 \mathrm{kV}$ with 0.1 psi head pressure. Melting points were acquired using Fisher-Johns melting point apparatus.

\subsection{Procedures for Synthesis of Monoalkoxyurea Thiol 1}

Monoalkoxyurea thiol 1 was prepared by Dr. Mandapati Raju (University of Louisville, Department of Chemistry).
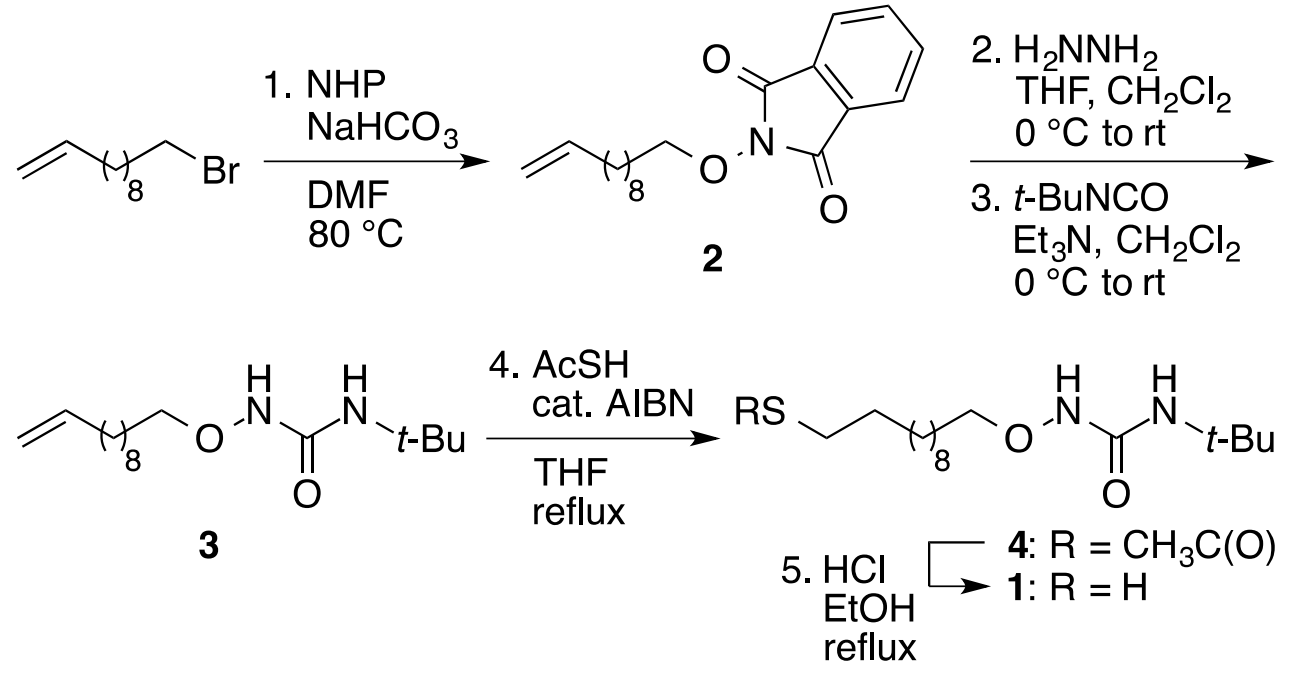

Figure 3.1 Synthetic route for preparation of monoalkoxyurea thiol 1 . NHP $=\mathrm{N}$ hydroxyphthalimide, AIBN= azoisobutyronitrile.

2-(Undec-10-en-1-yloxy)isoindoline-1,3-dione (2). A stirred solution of $N$ hydroxyphthalimide (NHP) $(5.56 \mathrm{~g}, 34.1 \mathrm{mmol})$ and sodium bicarbonate $(2.76 \mathrm{~g}, 32.9$ 
$\mathrm{mmol})$ in DMF $(60 \mathrm{~mL})$ was heated to $80{ }^{\circ} \mathrm{C}$. As the mixture turned dark brown, 11bromo-1-undecene $(2.83 \mathrm{~mL}, 12.9 \mathrm{mmol})$ was added and stirring was continued. After $10 \mathrm{~h}$, the reaction mixture was allowed to cool to room temperature and then poured into water $(60 \mathrm{~mL})$. The aqueous layer was extracted with ethyl acetate $(4 \mathrm{X}$ $60 \mathrm{~mL})$. The combined organic layer was washed with $5 \% \mathrm{LiCl}$ solution $(2 \mathrm{X} 50 \mathrm{~mL})$, dried $\left(\mathrm{MgSO}_{4}\right)$, filtered, and then concentrated by rotary evaporation. The crude product was purified by $\mathrm{SiO}_{2}$ column chromatography, eluting with a 15:85 mixture of EtOAc:hexane, to afford $3.51 \mathrm{~g}(86 \%)$ of $\mathbf{2}$ as a white solid; $\mathrm{mp}=35-37{ }^{\circ} \mathrm{C}$ (lit.[147] $\left.=38-40{ }^{\circ} \mathrm{C}\right) ; \mathrm{TLC}, \mathrm{R}_{f}=0.69$ (2.5: 7.5, EtOAc: hexane).

General hydrazinolysis and urea formation procedures: To a solution of phthalimide 2 (1.00 g, $3.17 \mathrm{mmol})$ in $\mathrm{CH}_{2} \mathrm{Cl}_{2}(10 \mathrm{~mL})$ at $0{ }^{\circ} \mathrm{C}$ was added hydrazine (10.2 $\mathrm{mL}$ of $1.0 \mathrm{M}$ solution in THF, $10.2 \mathrm{mmol})$. The reaction mixture was stirred at $0{ }^{\circ} \mathrm{C}$ for $1.5 \mathrm{~h}$ and then filtered. The retentate was washed with $\mathrm{CH}_{2} \mathrm{Cl}_{2}(10 \mathrm{~mL})$ and the combined organic solvents then were concentrated by rotary evaporation. The crude aminooxy product so obtained was used directly in the next step without further purification (Note: protect from carbonyl vapors, such as laboratory acetone).

To a stirred solution of the crude aminooxy $(0.58 \mathrm{~g}, 3.17 \mathrm{mmol})$ in $\operatorname{dry} \mathrm{CH}_{2} \mathrm{Cl}_{2}$ $(15 \mathrm{~mL})$ at room temperature under argon was added $\operatorname{dry}^{\mathrm{E}} \mathrm{E}_{3} \mathrm{~N}(0.48 \mathrm{~mL}, 3.45 \mathrm{mmol})$ via syringe. The reaction mixture was cooled to $0{ }^{\circ} \mathrm{C}$ and then $t$-butylisocyanate (0.32 $\mathrm{mL}, 2.87 \mathrm{mmol})$ was added dropwise over $5 \mathrm{~min}$. The reaction mixture was slowly allowed to reach room temperature and stirred $10 \mathrm{~h}$ before concentrating by rotary evaporation. The residue was purified by $\mathrm{SiO}_{2}$ flash column chromatography, eluting with a 1:4 mixture of EtOAc:hexane, to afford desired urea derivative. 
1-(tert-Butyl)-3-(undec-10-en-1-yloxy)urea (3): Using the general procedure outlined above, $2(1.00 \mathrm{~g}, 3.17 \mathrm{mmol})$ was transformed into 3, $0.62 \mathrm{~g}(68 \%)$ as a colorless liquid; TLC, $\mathrm{R}_{f}=0.51$ (2.5:7.5, EtOAc:hexane).

General thioacetate formation procedure: A solution of urea $3(0.5 \mathrm{~g}, 1.75$ mmol) and azobis(isobutyronitrile) $(0.043 \mathrm{~g}, 0.26 \mathrm{mmol})$ in dry THF $(20 \mathrm{~mL})$ was heated to reflux whereupon thioacetic acid $(0.25 \mathrm{~mL}, 3.51 \mathrm{mmol})$ was added via syringe. The reaction mixture was stirred at reflux $8 \mathrm{~h}$ and then concentrated by rotary evaporation. The residue was purified by $\mathrm{SiO}_{2}$ column chromatography, eluting with a 15:85 mixture of EtOAc:hexane, to afford thioacetate derivative.

\section{$S$-(11-((3-(tert-Butyl)ureido)oxy)undecyl) ethanethioate (4). Using a} general procedure outlined above, $3(0.50 \mathrm{~g}, 1.75 \mathrm{mmol})$ was transformed into thioester $4,0.48 \mathrm{~g}(75 \%)$ as a pale yellow liquid; TLC, $\mathrm{R}_{f}=0.42$ (2.5:7.5 EtOAc:hexane).

General thioacetate cleavage procedure: To a solution of thioester urea 4 $(0.50 \mathrm{~g}, 1.38 \mathrm{mmol})$ in $\mathrm{EtOH}(10 \mathrm{~mL})$ was added $12 \mathrm{~N} \mathrm{HCl}(1.1 \mathrm{~mL}, 11.0 \mathrm{mmol})$. The reaction mixture was heated to reflux and stirred. After $4 \mathrm{~h}$, the reaction mixture was cooled and then concentrated by rotary evaporation. The residue was dissolved in water $(10 \mathrm{~mL})$. The resultant solution was cooled to $0{ }^{\circ} \mathrm{C}$ and the $\mathrm{pH}$ was adjusted to slightly alkaline using saturated $\mathrm{NaHCO}_{3}$ solution. The aqueous layer then was extracted with $\mathrm{CH}_{2} \mathrm{Cl}_{2}(4 \mathrm{X} 30 \mathrm{~mL})$. The combined organic phase was washed with saturated $\mathrm{NaCl}(2 \mathrm{X} 20 \mathrm{~mL})$, dried $\left(\mathrm{MgSO}_{4}\right)$, filtered and concentrated under reduced pressure to afford monoalkoxyurea thiol 
1-(tert-Butyl)-3-((11-mercaptoundecyl)oxy)urea (1). Using the general procedure outlined above, $4(0.50 \mathrm{~g}, 1.38 \mathrm{mmol})$ was transferred into thiol urea 1 $(0.38 \mathrm{~g}, 86 \%)$ as a pale yellow liquid that was directly applied to gold nanoparticles; TLC, $\mathrm{R}_{f}=0.44$ (2.5:7.5 EtOAc:hexane).

\subsection{Synthesis of Gold Nanoparticles Functionalized with Monoalkoxyurea Thiol} 1.

The AuNP synthesis procedure described in Chapter 2 was applied here using the new thiol. First, $0.05 \mathrm{~g}$ of $\mathrm{HAuCl}_{4}$ was dissolved in $4 \mathrm{~mL}$ of deionized water; 0.08 $\mathrm{g}$ of TOAB was dissolved in $20 \mathrm{~mL}$ of toluene. Then the aqueous $\mathrm{HAuCl}_{4}$ solution was added to the toluene TOAB solution with vigorous stirring until all the tetrachloroaurate transferred into the organic phase. Next monoalkoxyurea thiol 1 (thiol: Au molar ratio of 1:1) was added in the above solution. An aqueous solution of $\mathrm{NaBH}_{4}$, freshly prepared by dissolving $0.056 \mathrm{~g} \mathrm{NaBH}_{4}$ in $4 \mathrm{~mL}$ deionized water, was slowly added with vigorous stirring to the toluene mixture. A rapid color change occurred as the $\mathrm{NaBH}_{4}$ solution was added. After stirring for at least 3 hours at room temperature, the black part was separated and dropwise added into $400 \mathrm{~mL}$ of ethanol with rapid stirring. The difference between monoalkoxyurea thiol and other thiols is that AuNPs stay in the middle phase of water layer and organic layer for monoalkoxyurea thiol, while AuNP stays in the organic phase for other commercial thiols. After settling overnight at freezer, the mixture was centrifuged and washed 
with ethanol for 3 times and dried at $40{ }^{\circ} \mathrm{C}$ in an oven to yield AuNPs (ca. $10 \mathrm{mg}$ ) suitable for sensor formation.

\section{Results and Discussion}

\subsection{NMR Characterization of as-Synthesized Alkoxyurea Thiol 1}

3.1.1 ${ }^{1} \mathrm{H}$ and ${ }^{13} \mathrm{C}$ NMR of 2-(Undec-10-en-1-yloxy)isoindoline-1,3-dione (2)

${ }^{1} \mathrm{H}$ NMR $\left(\mathrm{CDCl}_{3}, 400 \mathrm{MHz}\right) \delta 7.84-7.82(\mathrm{~m}, 2 \mathrm{H}), 7.75-7.73(\mathrm{~m}, 2 \mathrm{H})$, 5.81-

$5.79(\mathrm{~m}, 1 \mathrm{H}), 5.00-4.90(\mathrm{~m}, 2 \mathrm{H}), 4.19(\mathrm{t}, J=5.6 \mathrm{~Hz}, 2 \mathrm{H}), 2.04-2.00(\mathrm{~m}, 2 \mathrm{H}), 1.80-$

$1.76(\mathrm{~m}, 2 \mathrm{H}), 1.49-1.47(\mathrm{~m}, 2 \mathrm{H}), 1.36-1.29(\mathrm{~m}, 10 \mathrm{H}) \mathrm{ppm} ;{ }^{13} \mathrm{C} \mathrm{NMR}\left(\mathrm{CDCl}_{3}, 100\right.$ MHz) $\delta 163.7,139.2,134.5,129.1,123.5,114.2,78.7,33.9,29.5,29.4,29.3,29.2$, 29.0, 28.2, 25.6 ppm. IR 2923, 2851, 1787, 1730, 1464, 1399, $1186 \mathrm{~cm}^{-1}$.

${ }^{11} \mathrm{H}$ NMR $\left(400 \mathrm{MHz}, \mathrm{CDCl}_{3}\right)$ of 2

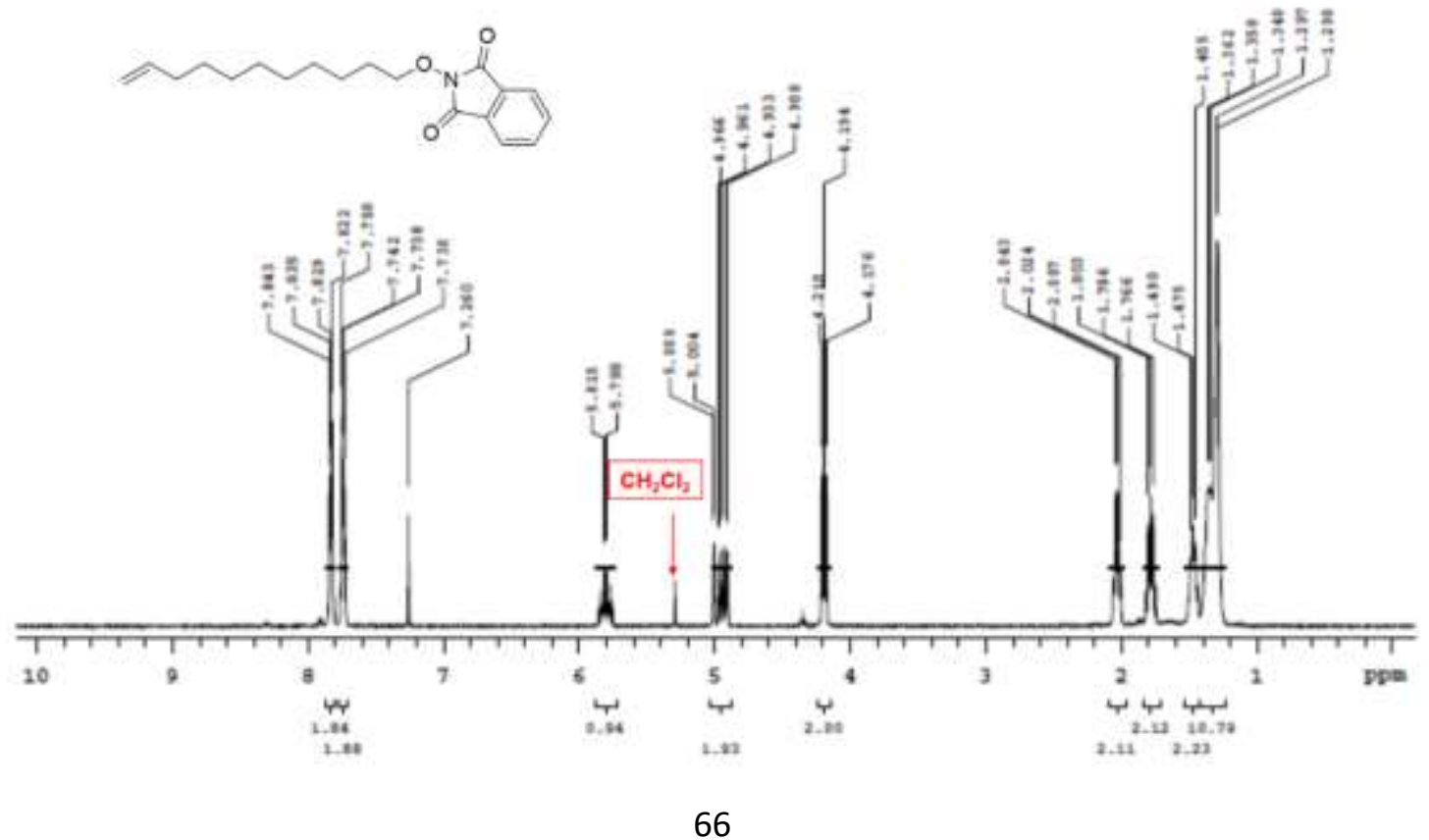


${ }^{13} \mathrm{C}$ NMR $\left(100 \mathrm{MHz}, \mathrm{CDCl}_{3}\right)$ of 2

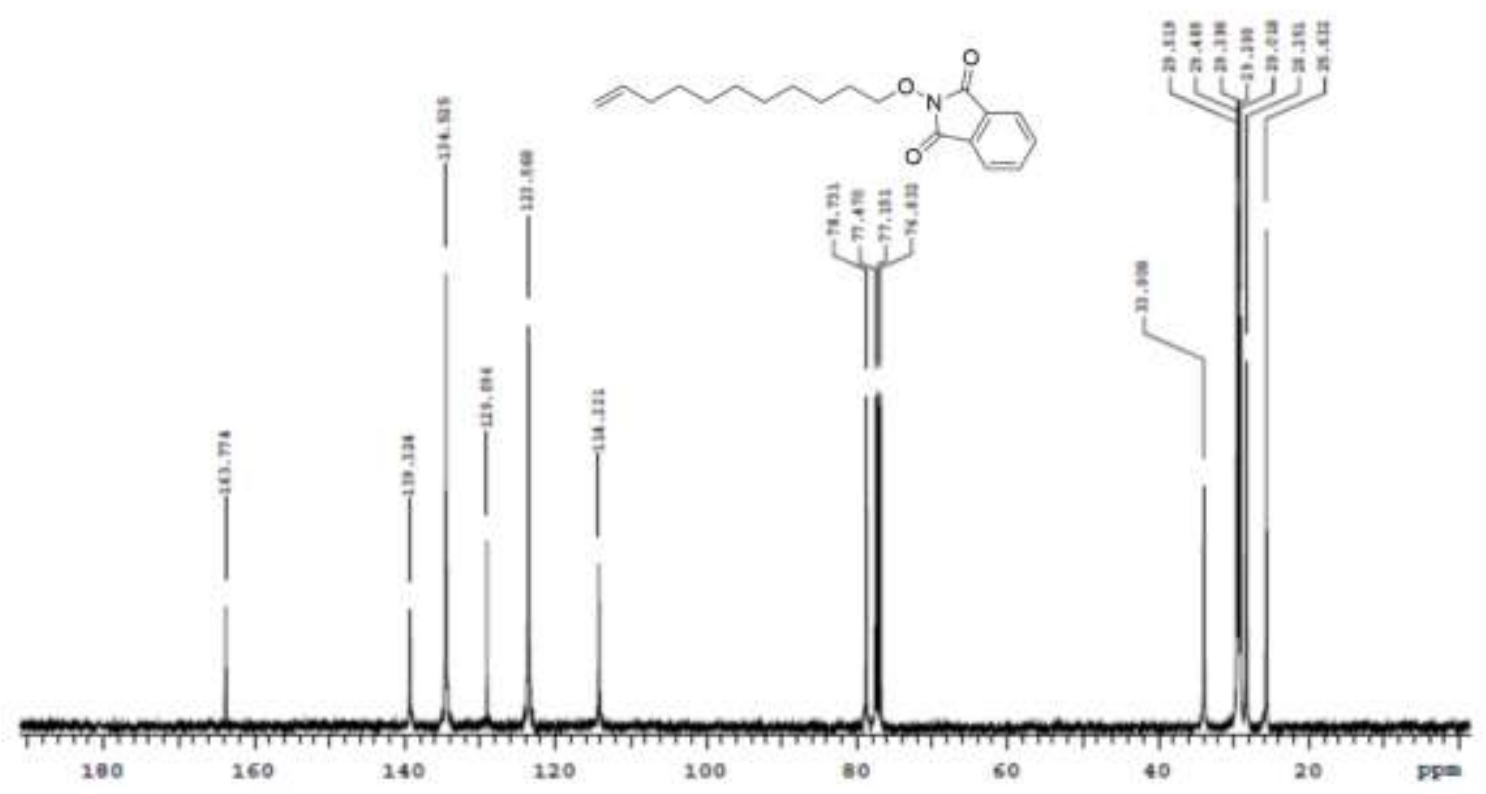

Figure 3.2 NMR spectra for ${ }^{1} \mathrm{H}$ NMR and ${ }^{13} \mathrm{C}$ NMR of 2.

3.1.2 ${ }^{1} \mathrm{H}$ and ${ }^{13} \mathrm{C}$ NMR of 1-(tert-Butyl)-3-(undec-10-en-1-yloxy)urea (3).

${ }^{1} \mathrm{H}$ NMR $\left(\mathrm{CDCl}_{3}, 400 \mathrm{MHz}\right) \delta 6.85(\mathrm{br}, \mathrm{NH}), 5.84-5.74(\mathrm{~m}, 1 \mathrm{H}), 5.59$ (br, NH), 5.00-4.90 (m, 2H), $3.77(\mathrm{t}, J=6.4 \mathrm{~Hz}, 2 \mathrm{H}), 2.05-2.00(\mathrm{~m}, 2 \mathrm{H}), 1.64-1.57(\mathrm{~m}$, $2 \mathrm{H}), 1.36-1.27(\mathrm{~m}, 21 \mathrm{H}) \mathrm{ppm} ;{ }^{13} \mathrm{C} \mathrm{NMR}\left(\mathrm{CDCl}_{3}, 100 \mathrm{MHz}\right) \delta$ 159.3, 139.1, 114.2, 76.4, 50.4, 33.8, 29.5, 29.4, 29.18, 29.12, 28.9, 28.1, 26.1 ppm; IR 3422, 3194, 2923, $2855,1674,1525,1457,1364,1235 \mathrm{~cm}^{-1}$; FT-ICR-MS for $\mathrm{C}_{16} \mathrm{H}_{32} \mathrm{~N}_{2} \mathrm{O}_{2}$ calculated 284.2463, found $285.3333[\mathrm{M}+\mathrm{H}]^{+}$. 
${ }^{1} \mathrm{H}$ NMR $\left(400 \mathrm{MHz}, \mathrm{CDCl}_{3}\right)$ of $\mathbf{3}$

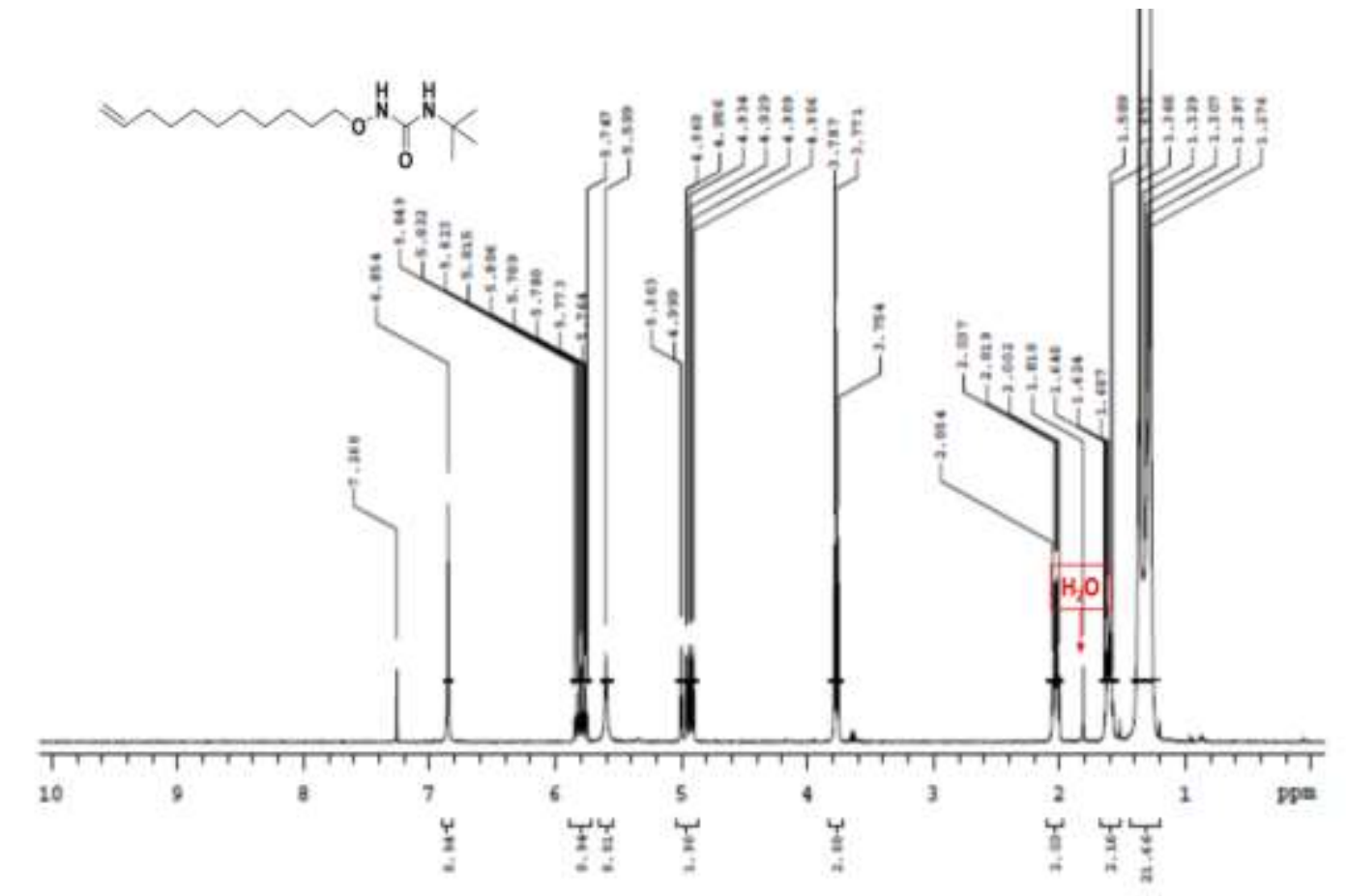

${ }^{13} \mathrm{C}$ NMR $\left(400 \mathrm{MHz}, \mathrm{CDCl}_{3}\right)$ of $\mathbf{3}$

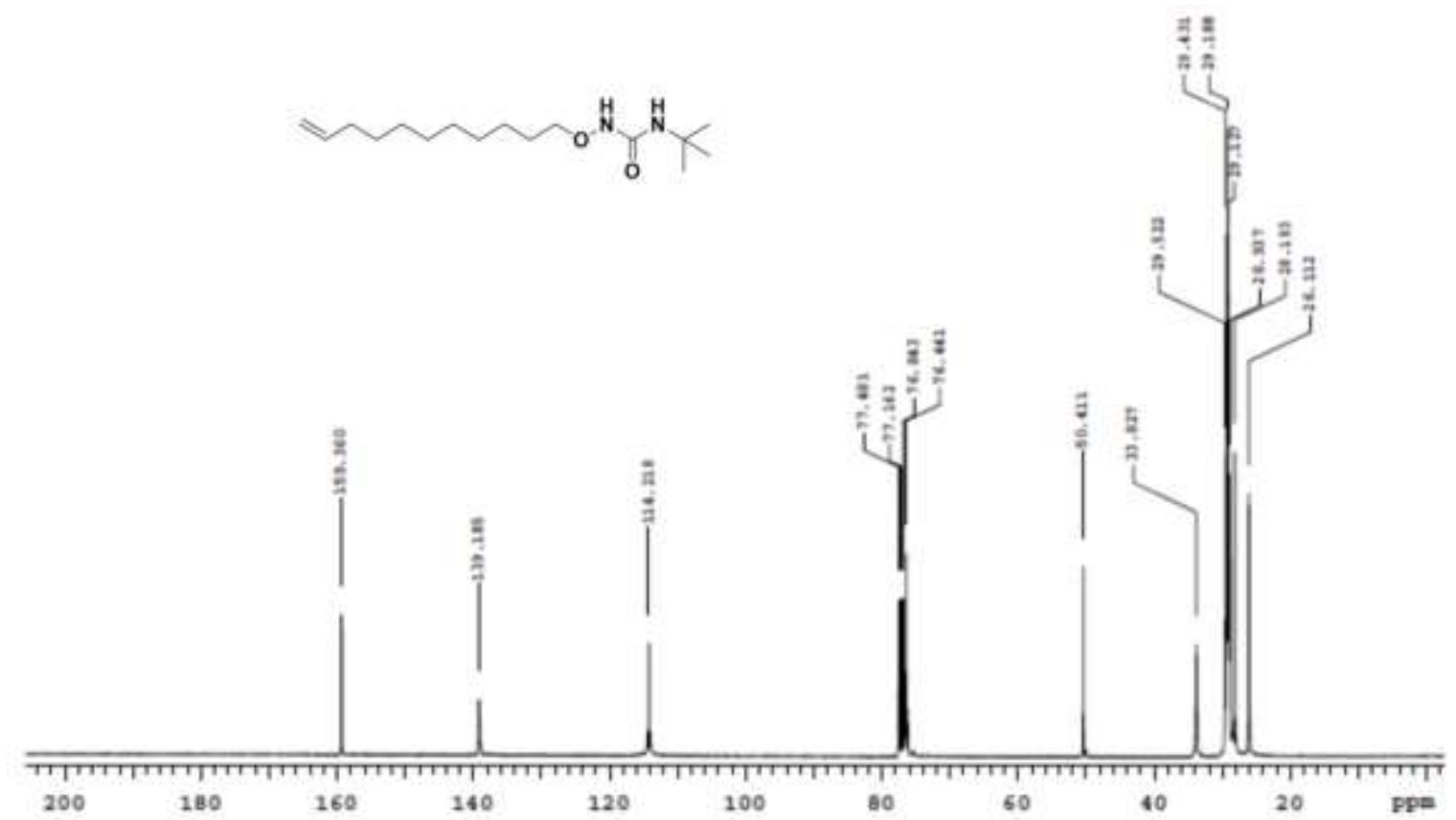

Figure 3.3 NMR spectra for $\mathrm{H}^{1} \mathrm{NMR}$ and ${ }^{13} \mathrm{C}$ NMR of 3 
3.1.3 ${ }^{1} \mathrm{H}$ and ${ }^{13} \mathrm{C}$ NMR of $S$-(11-((3-(tert-Butyl)ureido)oxy)undecyl) ethanethioate (4) ${ }^{1} \mathrm{H}$ NMR (CDCl $\left.3,400 \mathrm{MHz}\right) \delta 6.91(\mathrm{br}, \mathrm{NH}), 5.59(\mathrm{br}, \mathrm{NH}), 3.76(\mathrm{t}, J=6.8$ $\mathrm{Hz}, 2 \mathrm{H}), 2.84(\mathrm{t}, J=6.8 \mathrm{~Hz}, 2 \mathrm{H}),, 2.30(\mathrm{~s}, 3 \mathrm{H}), 1.59-1.50(\mathrm{~m}, 4 \mathrm{H}), 1.35-1.25(\mathrm{~m}$, 23H), ppm; ${ }^{13} \mathrm{C} \mathrm{NMR}\left(\mathrm{CDCl}_{3}, 100 \mathrm{MHz}\right) \delta 196.1,159.2,76.4,50.5,30.7,30.6,29.5$, 29.2, 28.8, 28.2, $26.1 \mathrm{ppm}$; IR 3427, 2925, 2854, 1689, $1673 \mathrm{~cm}^{-1}$; FT-ICR-MS for $\mathrm{C}_{18} \mathrm{H}_{36} \mathrm{~N}_{2} \mathrm{O}_{2} \mathrm{~S}$ calculated 360.2446, found $361.25001[\mathrm{M}+\mathrm{H}]^{+}$.

${ }^{1} \mathrm{H}$ NMR $\left(400 \mathrm{MHz}, \mathrm{CDCl}_{3}\right)$ of 4

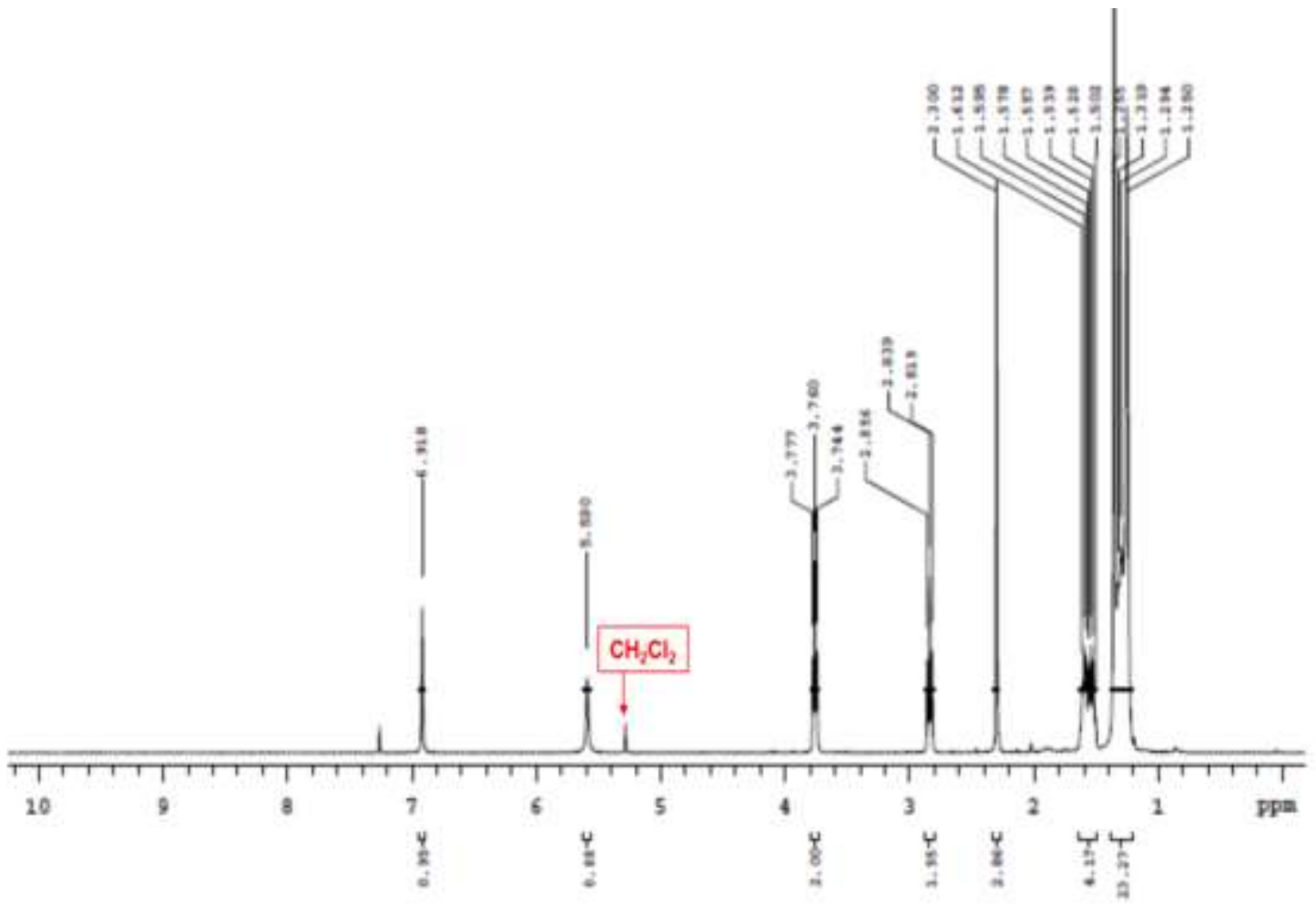


${ }^{13} \mathrm{C} \mathrm{NMR}\left(400 \mathrm{MHz}, \mathrm{CDCl}_{3}\right)$ of 4

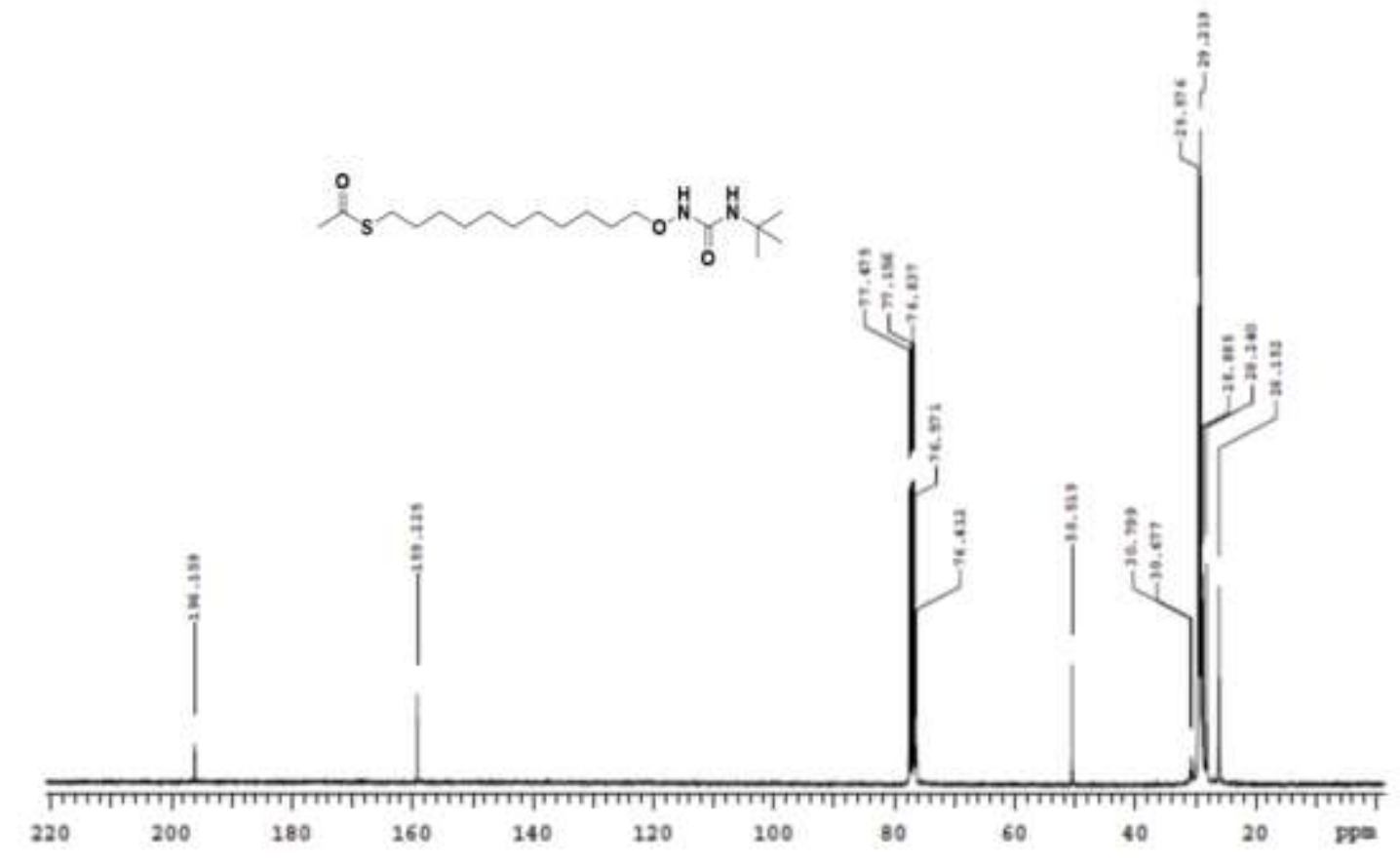

Figure 3.4 NMR spectra for ${ }^{1} \mathrm{H}$ NMR and ${ }^{13} \mathrm{C}$ NMR of 4

3.1.4 ${ }^{1} \mathrm{H}$ and ${ }^{13} \mathrm{C}$ NMR of 1-(tert-Butyl)-3-((11-mercaptoundecyl)oxy)urea (1)

${ }^{1} \mathrm{H} \mathrm{NMR}\left(\mathrm{CDCl}_{3}, 400 \mathrm{MHz}\right) \delta 6.76(\mathrm{br} \mathrm{s}, 1 \mathrm{H}, \mathrm{NH}), 5.60(\mathrm{br} \mathrm{s}, 1 \mathrm{H}, \mathrm{NH}), 3.77$ $(\mathrm{t}, J=6.8 \mathrm{~Hz}, 2 \mathrm{H}), 2.51(\mathrm{q}, J=7.4 \mathrm{~Hz}, 2 \mathrm{H}), 1.61-1.55(\mathrm{~m}, 4 \mathrm{H}), 1.36-1.26(\mathrm{~m}, 23 \mathrm{H}, \mathrm{t}-$ butyl + aliphatic chain) ppm; ${ }^{13} \mathrm{C} \mathrm{NMR}\left(\mathrm{CDCl}_{3}, 100 \mathrm{MHz}\right) \delta 159.2,76.4,50.4,34.0$, 29.5, 29.4, 29.2, 29.1, 28.3, 28.2, 26.1, 24.6 ppm; IR 3426, 2924, 2853, 1689, 1672, $1525 \mathrm{~cm}^{-1}$; FT-ICR-MS for $\mathrm{C}_{16} \mathrm{H}_{34} \mathrm{~N}_{2} \mathrm{O}_{2} \mathrm{~S}$ calcd 318.2341, found 319. $2419[\mathrm{M}+\mathrm{H}]^{+}$. 
${ }^{1} \mathrm{H}$ NMR $\left(400 \mathrm{MHz}, \mathrm{CDCl}_{3}\right)$ of $\mathbf{1}$<smiles>CC(C)(C)NC(=O)NCOCCCCCCCCCCCS</smiles>

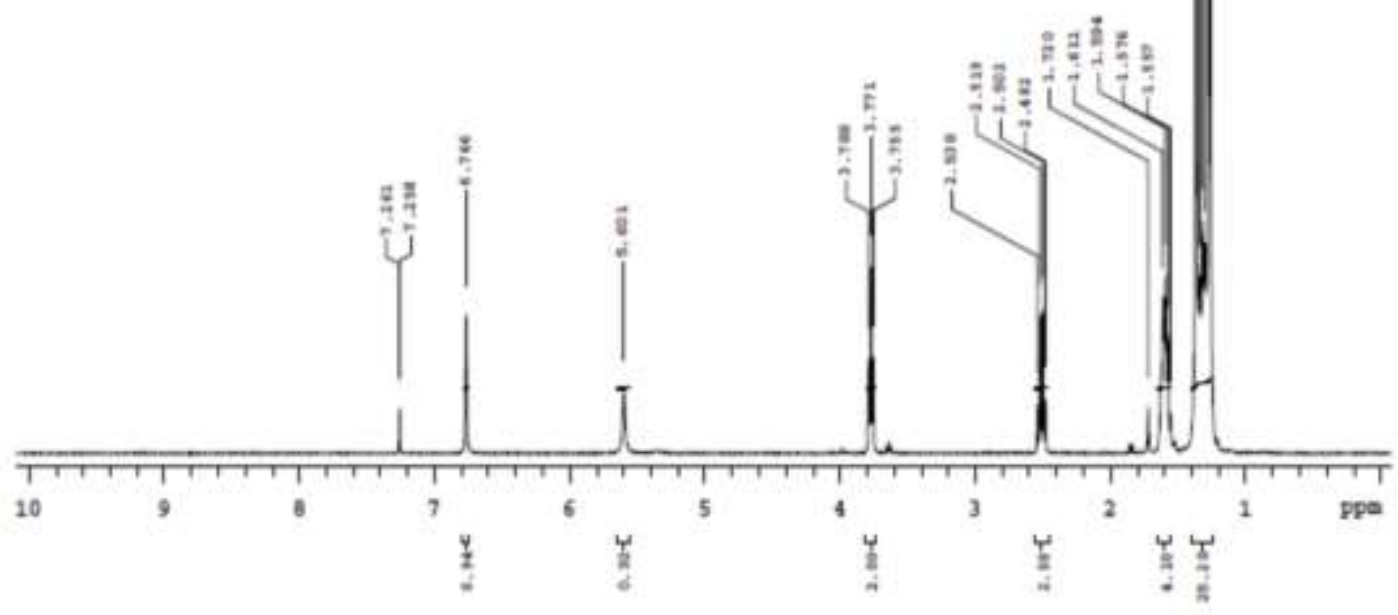

${ }^{13} \mathrm{C}$ NMR (400 MHz, $\mathrm{CDCl}_{3}$ ) of $\mathbf{1}$

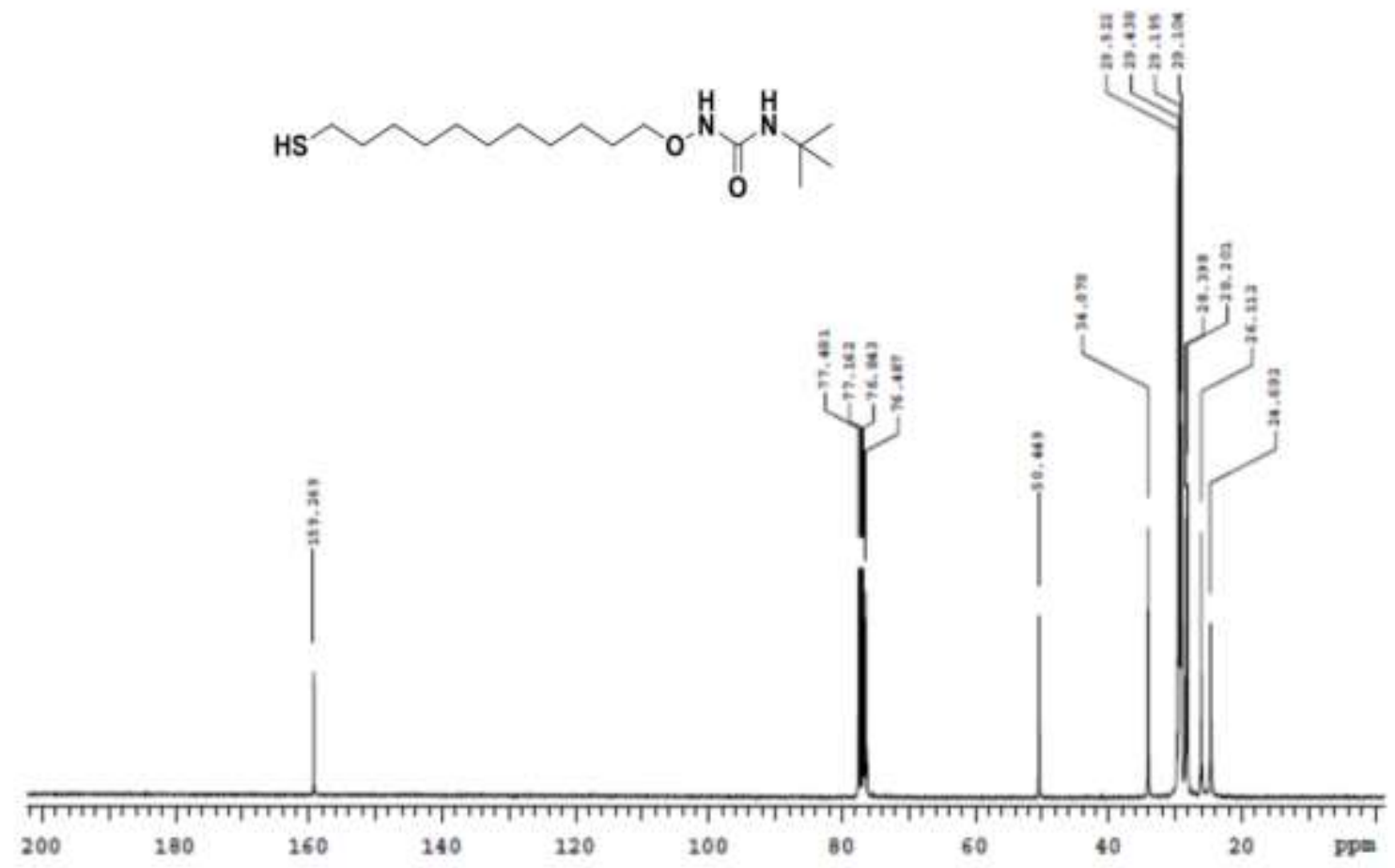

Figure 3.5 NMR spectra for ${ }^{1} \mathrm{H}$ NMR and ${ }^{13} \mathrm{C}$ NMR of 1 . 


\subsection{Characterization of AuNP Functionalized with Monoalkoxyurea Thiol 1}
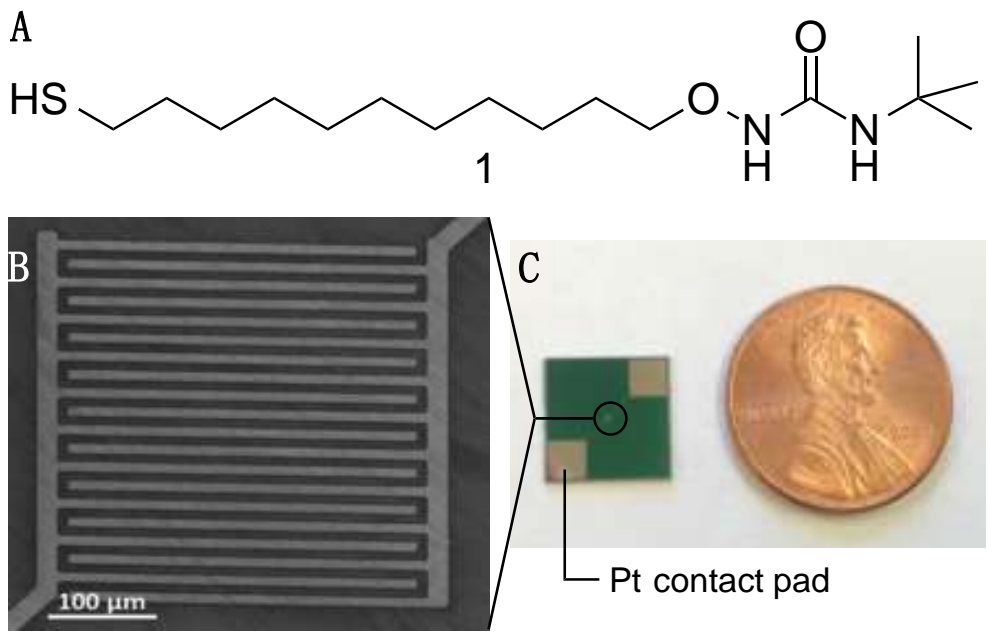

Figure 3.6 A: Structure of thiol 1; B: SEM micrograph of the microfabricated, interdigitated electrode; $\mathrm{C}$ : size comparison between the sensor connected to platinum contact pads and a one-cent coin.

Figure 3.6A shows the structure of thiol 1 we designed to compare with thiols featuring different organic functionalities, namely 1-dodecanethiol, 4-methoxy- $\alpha$ toluenethiol, and 11-mercaptoundecanoic acid. Figure 3.6B depicts the interdigitated electrodes at the core of the sensor. Figure $3.5 \mathrm{C}$ shows that the overall size of the sensor is rather small in comparison to a one-cent coin. 

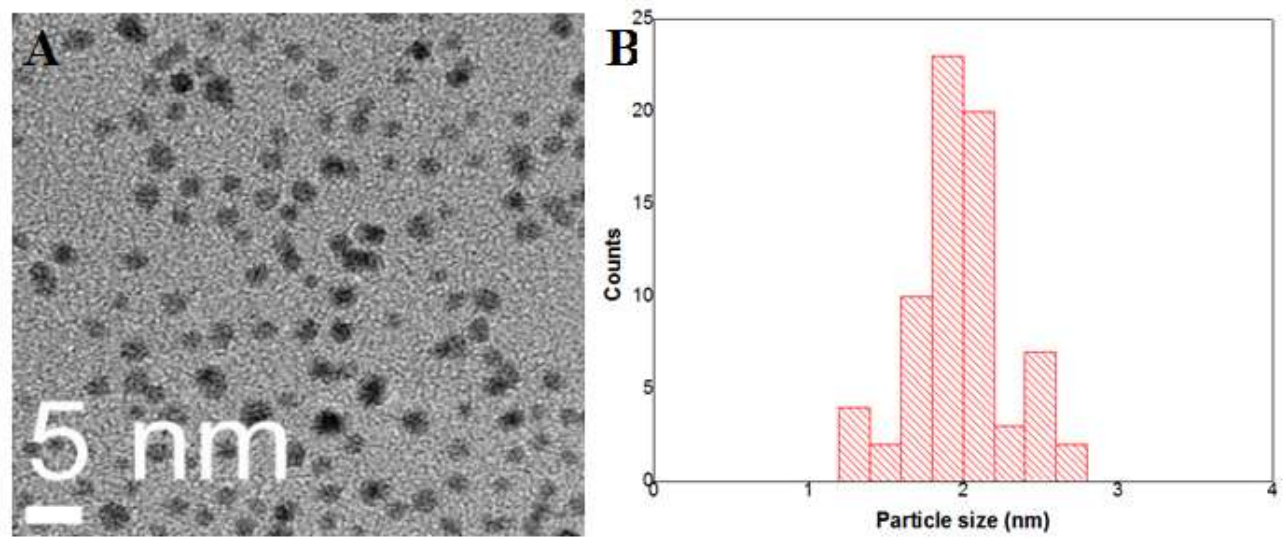

Figure 3.7 A: TEM of AuNP functionalized with thiol 1; B: histogram of AuNP size distribution.

Figure 3.7 shows the TEM image of AuNP functionalized with monoalkoxyurea thiol 1. The sizes of the thiol functionalized AuNPs ranged from 1-3 nm, which is consistent with thiol monolayer results reported by Brust [122]. Figure 3.7A shows that the AuNPs are uniform and well dispersed. 


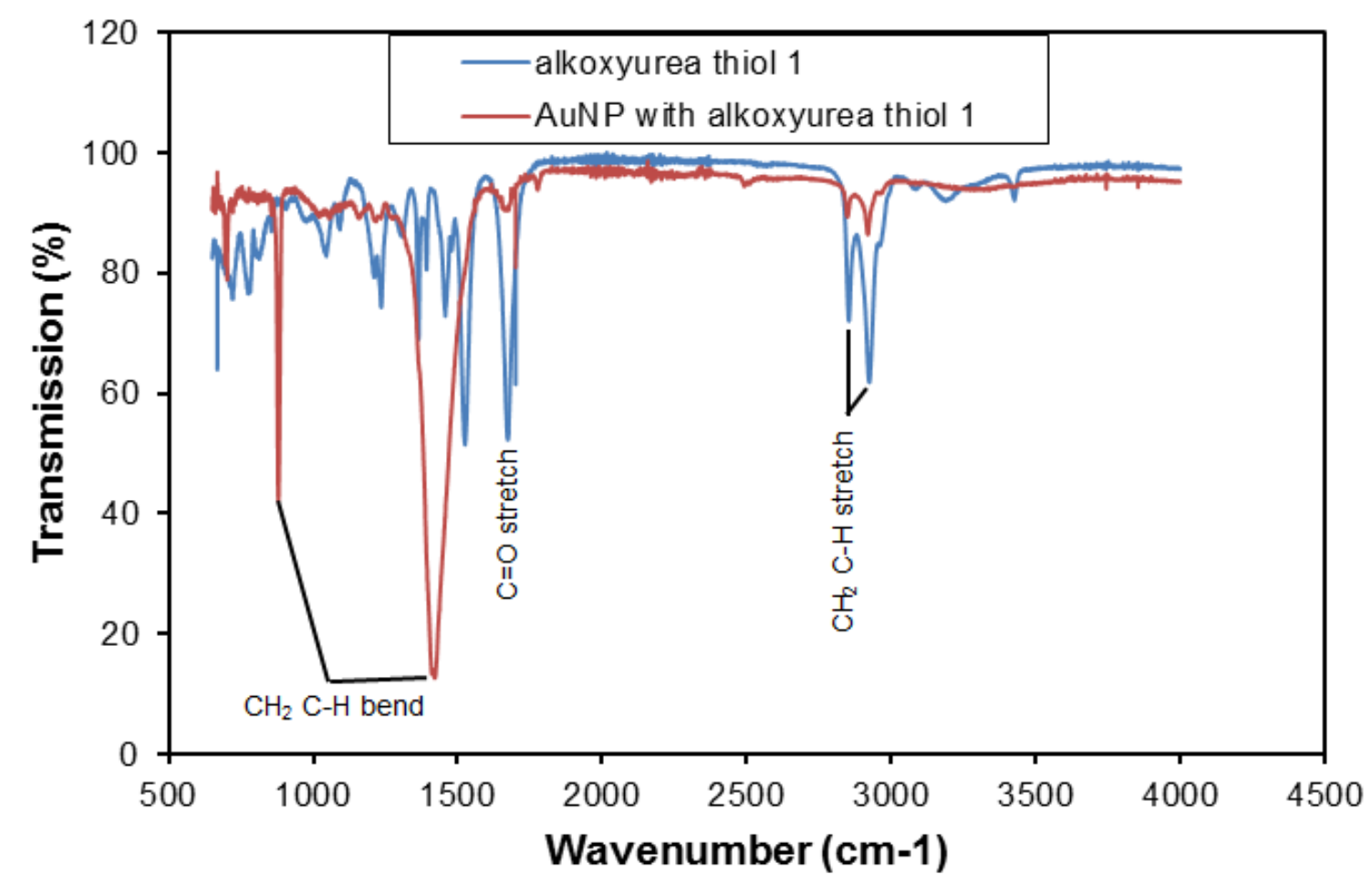

Figure 3.8 IR spectra of thiol 1 and thiol 1-functionalized AuNPs prepared in this work.

Figure 3.8 shows the IR spectra of monoalkoxyurea thiol 1 and AuNP functionalized with urea thiol 1. Even though the intensity of the peaks is different, the wavenumbers of the peaks are similar, which means the AuNP is successfully functionalized with urea thiol 1. TGA of the AuNP with urea thiol 1 is shown in Figure 3.9. In the urea thiol 1 functionalized AuNP material, the first weight loss was observed to take place at $\sim 170-180{ }^{\circ} \mathrm{C}(\sim 14 \%$ weight loss $)$ while the second weight loss was observed to take place at $\sim 280{ }^{\circ} \mathrm{C}$ ( $\sim 28 \%$ weight loss), corresponding to a total weight loss of $\sim 42 \%$. The first weight loss region was responsible for water loss. The second weight loss was the thermal decomposition of the urea thiol 1 coating on gold nanoparticles. 


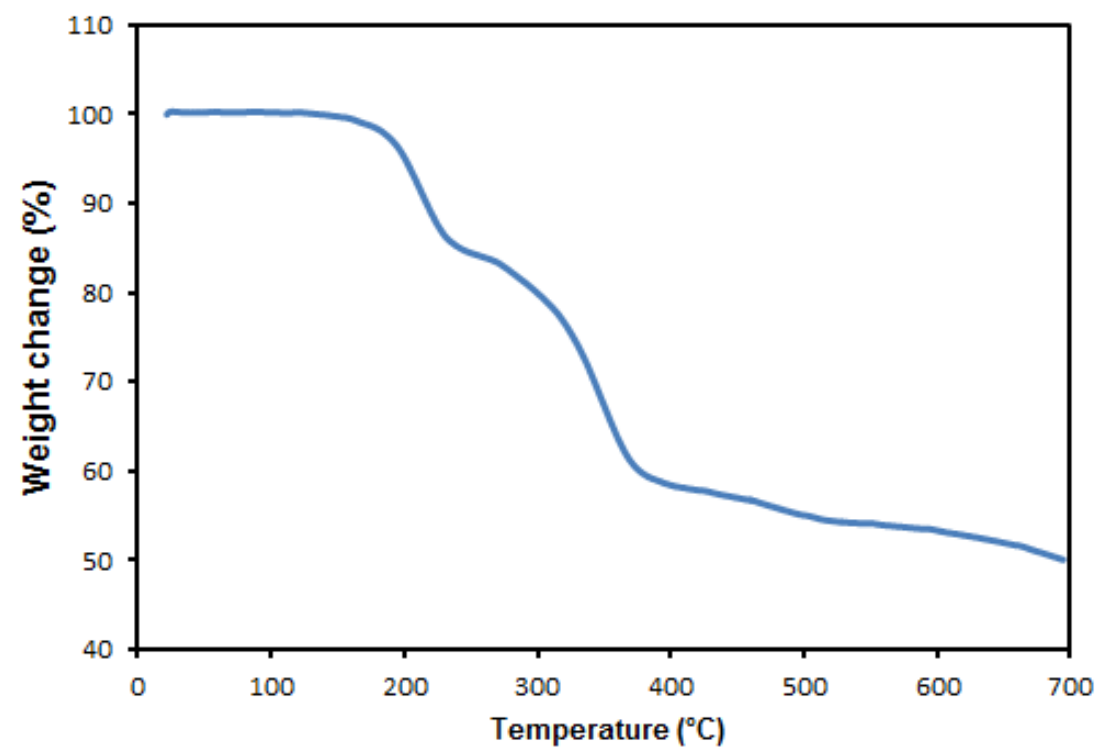

Figure 3.9 TGA of AuNP with monoalkoxyurea thiol 1.

\subsection{VOC Sensing Properties of Alkoxyurea Thiol Gold Nanoparticles}

The AuNPs were subsequently used for sensor fabrication. Alternating cycles of VOC introduction into the chamber followed by chamber evacuation were performed while sensor resistance was monitored and recorded as a function of time. Figure 3.10 shows the response profile for the urea thiol 1 AuNP sensor upon repeated exposures to $100 \mathrm{ppb}$ acetone. The resistance decreases in the presence of acetone and return to baseline value upon chamber evacuation. The sensor response time at this concentration is $2 \mathrm{~s}$ and recovery time is $6 \mathrm{~s}$. These results show a rapid and fully reversible interaction of analyte with the sensing structure and that the maximum response value remains similar over multiple cycles of exposure to the same 
concentration of gases, confirming that the construct can be used multiple times for sensing.

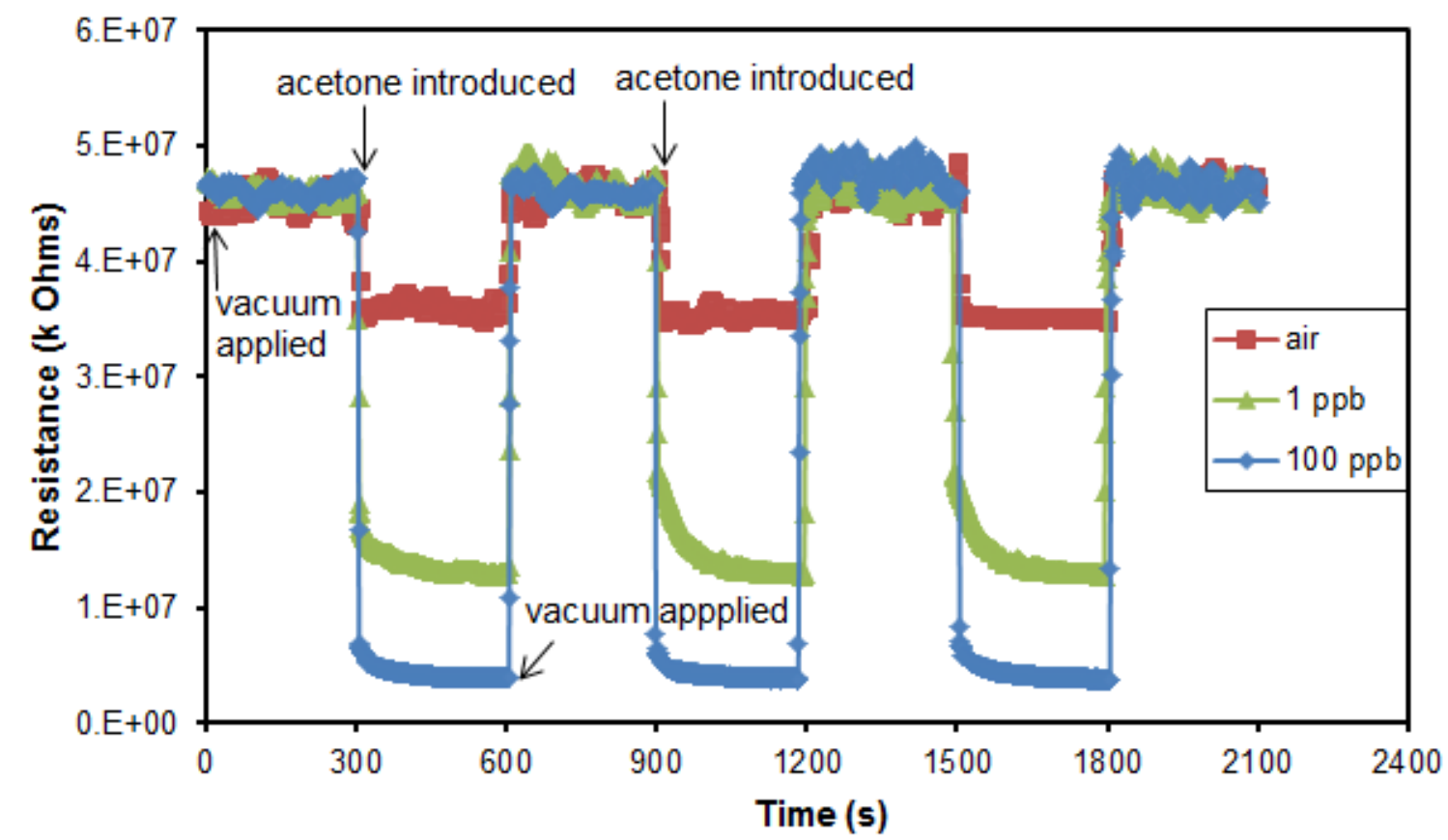

Figure 3.10 Response profile of thiol 1 AuNP sensor on repeated exposure to air, 1 ppb and $100 \mathrm{ppb}$ acetone

To further examine responses to trace levels of acetone, we tested our monoalkoxyurea thiol 1 AuNP sensor over a wide concentration range of acetone (Figure 3.11A). To our delight, the thiol 1 AuNP sensor was sensitive toward acetone at concentrations as low as $0.1 \mathrm{ppb}$, a level of detection far superior to other acetone tracing devices. Furthermore, the response increased with increasing acetone concentration up to $1000 \mathrm{ppm}$, thus demonstrating a wide dynamic range of measurements. Given this high sensitivity toward acetone, we set out to test the carbonyl-sensing selectivity of the sensor by measuring sensor responses to non- 
carbonyl VOCs (Figure 3.11B). As the slope of the linear regression curve is a direct measure of sensitivity, the relatively flat slopes obtained for the non-carbonyl analytes (all slopes below 1.6) imply the sensor discriminates in its interactions with VOCs. The considerably stronger response to acetone (slope=4.6) suggests the hydrogen bonding design concept is operative and imparts both sensitivity and selectivity.
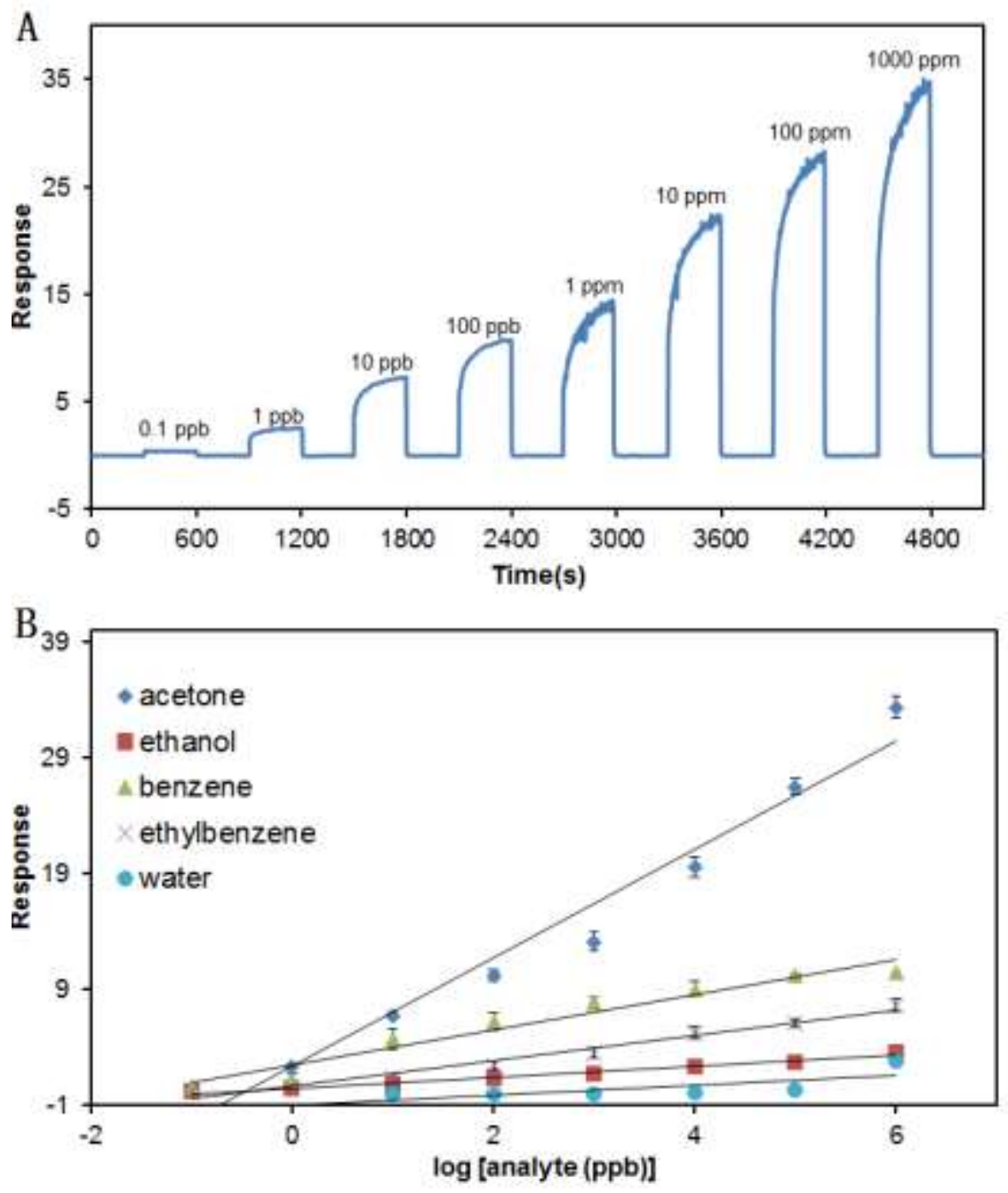

Figure 3.11 A: Response of the thiol 1 AuNP sensor to acetone exposure at $0.1 \mathrm{ppb}$ to $1000 \mathrm{ppm}$; B: sensor response versus $\log [$ analyte $(\mathrm{ppb})]$ in detection of acetone, 
ethanol, benzene, ethylbenzene and water vapor. The responses are nearly linear $\left(\mathrm{R}^{2}>0.97\right)$. Error bars indicate the variability among three different sensors. Most error bars are smaller than the data symbols.

\subsection{Comparison of Monoalkoxyurea Thiol 1-AuNP and Commercial Thiol-AuNP} Sensors

To further escalate the role of the $\mathrm{H}$-bonding motif in providing chemoselection for a carbonyl moiety, we compared the responses of sensors prepared using AuNPs functionalized with the commercial thiols 1-dodecanethiol (sensor a) , 4-methoxy- $\alpha$ toluenethiol (sensor b) and 11-mercapto-undecanoic acid (11-MUA) (sensor c) (Figure 3.12). These three thiol-derived AuNP sensors respond to acetone vapor with considerably lower sensitivities than the thiol 1 (sensor d) AuNP sensor (Figure 3.13). Indeed, at the general concentration of acetone in exhaled breath $-1 \mathrm{ppm}-$ the response of the thiol 1 AuNP sensor was $~ 10,12$ and 23 times higher than the AuNP sensors derived from DDT, MTT and 11-MUA, respectively. These results strongly support the idea that incorporation of terminal functionality on a thiol monolayer surrounding AuNPs can be used to confer selectivity in analyte detection. Along these lines, the sensor derived from MTT responded selectively to ethanol, albeit at a substantially lower response relative to the thiol 1-derived sensor in its detection of acetone. 


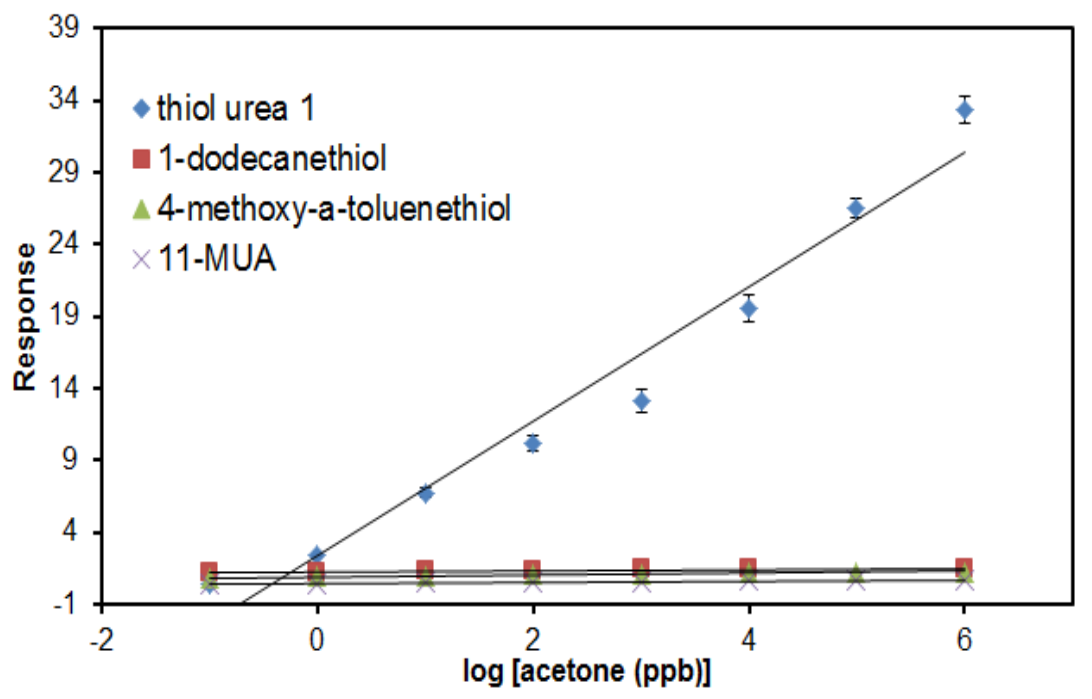

Figure 3.12 Responses for sensors derived from AuNPs coated with thiol urea 1, 1dodecanethiol 4-methoxy- $\alpha$-toluenethiol or 11-mercaptoundecanoic acid in detection of acetone.

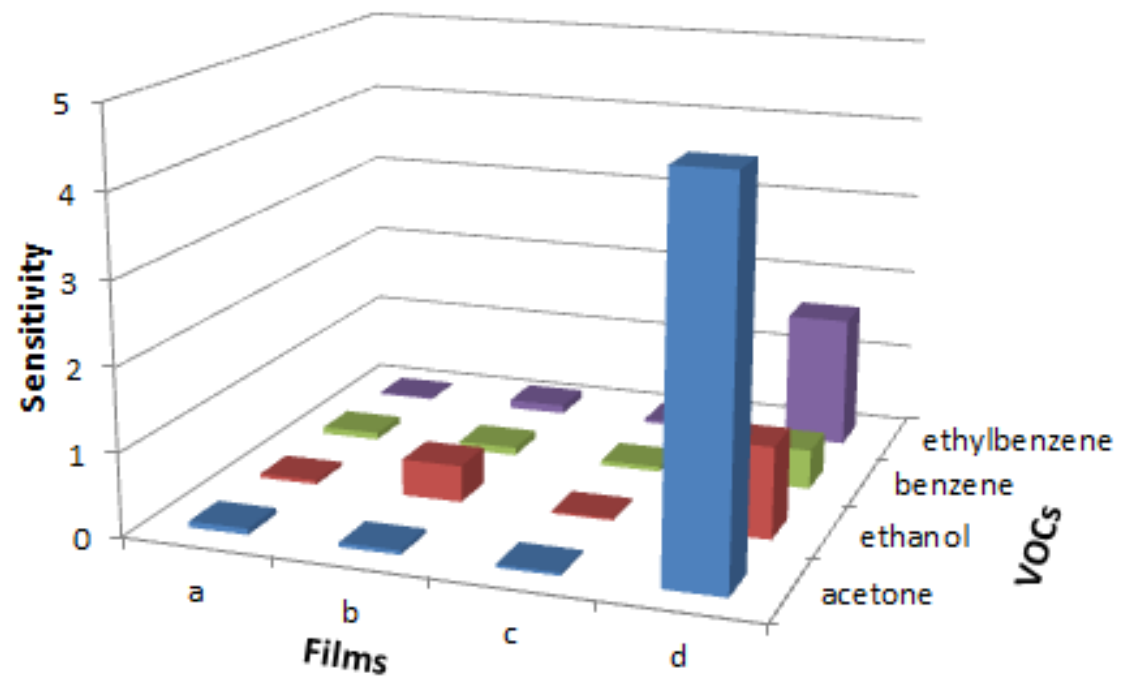

Figure 3.13 A comparison of the response sensitivities obtained for the four vapors on the four-sensor array system. 
On comparing the acetone sensing performance of the thiol 1-derived sensor to other chemiresistors reported in the literature (Table 6), we noted differences in several key parameters that underscore the advantages of the present design. First, an operating temperature under ambient conditions is preferable. A measurable response with fast response and recovery times obviously are also needed for clear and reversible analyte detection. Finally, a low ppm limit of detection is needed for challenging analytical applications, such as in breath analysis. We were gratified to note that the thiol 1-derived sensor met all criteria and was superior in nearly every category for detection of acetone.

Table 6 A comparison of chemiresistor parameters and limits for detection of acetone $^{a}$

\begin{tabular}{|c|c|c|c|c|c|c|}
\hline Sensing material & $\begin{array}{c}\text { Tem } \\
\text { p. } \\
\left({ }^{\circ} \mathrm{C}\right)\end{array}$ & $\begin{array}{l}\text { Acetone } \\
\text { Conc. }\end{array}$ & Response & $\begin{array}{c}\mathbf{T}_{\mathbf{s}} / \mathbf{T}_{\mathbf{c}}{ }^{b} \\
(\mathbf{s})\end{array}$ & LoD & Ref. \\
\hline $\begin{array}{c}\mathrm{WO}_{3} \text { functionalized } \\
\text { with AuNPs }\end{array}$ & 220 & $0.20 \%$ & 3.5 & $217 / 400$ & N/A & {$[65]$} \\
\hline $\begin{array}{c}\mathrm{SnO}_{2}-\mathrm{ZnO} \text { hetero- } \\
\text { nanofibers }\end{array}$ & 300 & $100 \mathrm{ppm}$ & 34 & $25 / 9$ & $5 \mathrm{ppm}$ & {$[51]$} \\
\hline $\begin{array}{c}\text { 3D- }-\mathrm{TiO}_{2} \\
\text { nanoflowers }\end{array}$ & 60 & 700 ppm & $2^{c}$ & $10 / 45$ & $1 \mathrm{ppm}$ & {$[52]$} \\
\hline $\begin{array}{c}\text { Ag-doped } \\
\mathrm{TiO}_{2} / \mathrm{SnO}_{2}\end{array}$ & 275 & $50 \mathrm{ppm}$ & 13 & $28 / 34$ & $1 \mathrm{ppm}$ & {$[53]$} \\
\hline Cr-doped $\mathrm{ZnO}$ & 300 & 1000 ppm & $19^{c}$ & $70 / 95$ & $15 \mathrm{ppm}$ & [54] \\
\hline $\begin{array}{c}\text { MAPLE-deposited } \\
\mathrm{TiO}_{2} \mathrm{NP}\end{array}$ & 400 & 100 ppm & $5^{c}$ & $\begin{array}{l}240 / \\
\text { N/A }\end{array}$ & $20 \mathrm{ppm}$ & {$[55]$} \\
\hline $\mathrm{Si}$-doped $\mathrm{WO}_{3} \mathrm{NP}$ & 400 & $600 \mathrm{ppb}$ & 4.5 & N/A & $20 \mathrm{ppb}$ & [49] \\
\hline $\mathrm{SnO}_{2}$ nanowires & 290 & $20 \mathrm{ppm}$ & 5.5 & $7 / 10$ & $20 \mathrm{ppm}$ & {$[50]$} \\
\hline $\begin{array}{l}\text { SWNT coated on } \\
\text { PET }\end{array}$ & $\mathrm{RT}$ & $10000 \mathrm{ppm}$ & $\begin{array}{l}0.22 \pm \\
0.024\end{array}$ & N/A & $10 \mathrm{ppb}$ & [148] \\
\hline
\end{tabular}




\begin{tabular}{|c|c|c|c|c|c|c|}
\hline PANI/SnO ${ }_{2}$ hybrid & 90 & 800 ppm & 1.45 & $\begin{array}{l}16-20 / \\
35-48\end{array}$ & $\begin{array}{c}500 \\
\mathrm{ppm}\end{array}$ & [56] \\
\hline $\mathrm{Co}_{3} \mathrm{O}_{4}$ & N/A & $1000 \mathrm{ppm}$ & 7.5 & $1-3 / 4-8$ & $10 \mathrm{ppm}$ & [57] \\
\hline $\mathrm{TiO}_{2}$ & 500 & 7.5 ppm & 14 & $380 / 300$ & $1 \mathrm{ppm}$ & [58] \\
\hline $\mathrm{CeO}_{2}$ doped $\mathrm{ZnO}$ & 320 & 100 ppm & 19 & $10 / 5$ & N/A & [59] \\
\hline thiol 1 AuNPs & RT & $1000 \mathrm{ppm}$ & $25.5^{\mathrm{c}}$ & $2 / 7$ & $0.1 \mathrm{ppb}$ & $\begin{array}{c}\text { present } \\
\text { work }\end{array}$ \\
\hline
\end{tabular}

${ }^{a} 3 \mathrm{D}=3$ dimensional, MAPLE $=$ matrix assisted pulsed laser evaporation, $\mathrm{NP}=$ nanoparticle, $\mathrm{PET}=$ poly (ethyleneterephthalate $), \mathrm{PANI}=$ polyaniline, $\mathrm{N} / \mathrm{A}=$ not available, $\mathrm{RT}=$ room temperature, $\mathrm{LoD}=$ limit of detection; ${ }^{b}$ response time $\left(\mathrm{T}_{\mathrm{s}}\right)$ and recovery time $\left(\mathrm{T}_{\mathrm{c}}\right) ;{ }^{c}$ calculated from published results for Response $=\mathrm{R}_{\text {air }} / \mathrm{R}_{\mathrm{gas}}-1$.

How does the alkoxyurea-motif interact with other carbonyls common in exhaled breath? Intrigued by this question, we next sought to investigate the response of current monoalkoxyurea thiol 1 AuNP sensor towards carbonyls other than acetone. As shown in Figure 3.14, the alkoxyurea motif of the sensor appears well suited for detecting carbonyl VOCs other than acetone, a feature confirmed by the high responses of the monoalkoxyurea thiol 1-derived sensor to carbonyl VOCs 2butanone, acrolein, and acetaldehyde. Interestingly, while still substantial, the response to 2-butanone, a methylene homolog of acetone, is lower relative to acetone by as much as $50 \%$. This level of sensitivity to a change as subtle as a single methylene group suggests fine-tuning of the structural environment surrounding the urea moiety may be used to achieve sensor selectivity. 


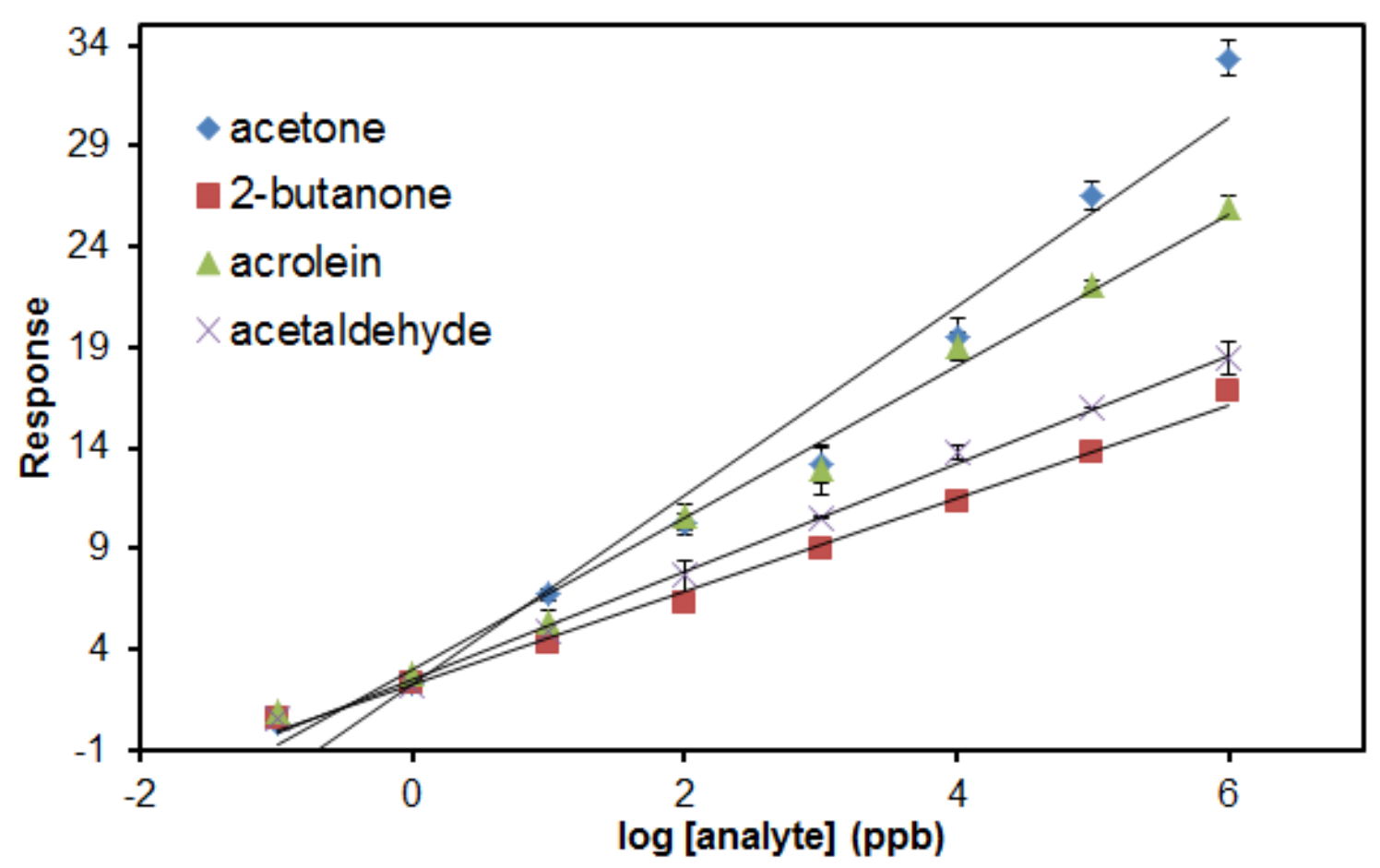

Figure 3.14 Responses of monoalkoxyurea thiol 1 AuNP sensor in detecting different carbonyl compounds. Error bars indicate the variability among three different sensors

\subsection{Long-term Stability of the Sensors}

The long-term stability of the sensor is a critical consideration in order to be applied in real world settings. Figure 3.15 shows the long-term stability of monoalkoxyurea thiol 1 AuNP sensor exposed to $1 \mathrm{ppm}$ acetone gas. The measurements were taken over 30 days and the responses were observed over different days. The response varied; however, no significant changes were observed during the first 15 days. After 15 days, the response values of the sensor began to decrease, likely due to the thiol ligand oxidation under ambient conditions $[149,150]$. The thiol molecules are prone to oxidation by $\mathrm{O}_{2}$ when exposed to air, forming 
sulfonyl-like oxidized products. The oxidized ligands cannot protect the $\mathrm{Au}$ core anymore and the sulfonyl species may desorb from the particle surface, which causes the gold nanoparticles to aggregate. The sensing film is much more conductive for the aggregated nanoparticles compared to the fresh film, resulting in severe deterioration of the sensor performance.

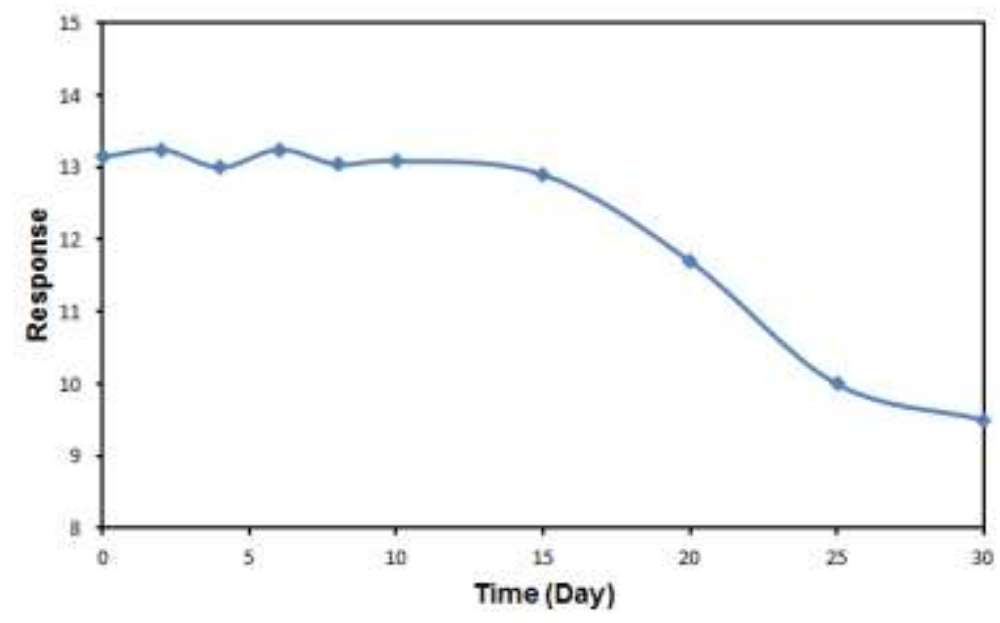

Figure 3.15 Stability of monoalkoxyurea thiol 1 sensor on exposure to $1 \mathrm{ppm}$ acetone over 30 days

\section{Conclusion}

In summary, the designed urea-functionalized thiol AuNP sensor provided high sensitive and selective response to acetone in reversible and reproducible manner at ambient temperature. Gas sensing measurements reveal that this sensor shows the lowest limit of detection to acetone among the reported chemiresistors to date. The stability of the AuNP sensor ( $>15$ days) suggests potential applications as a component of an electronic nose for analysis of VOCs. The concept of designed thiols 
for selective interaction with target analytes based on functional group interactions, such as urea-carbonyl hydrogen bonding, may well serve as a powerful strategy to improve chemiresistor sensitivity and selectivity in detection of other VOC classes. 


\section{CHAPTER IV}

\section{ELECTRONIC NOSE FOR DETECTION ACETONE IN EXHALED BREATH}

\section{Introduction}

Diabetes is a metabolic disease in which the pancreas does not produce enough insulin or cells do not respond properly to the insulin produced. Insulin is a peptide hormone produced by the pancreas that allows the body to convert sugar (glucose) from carbohydrates in the food into energy needed for daily life or to store glucose for the future use. Insulin helps keep the blood sugar level from getting too high or too low. The cause of diabetes varies depending on the genetic makeup, family history, ethnicity, health and environmental factors, such as obesity and lack of exercise. There are three main types of diabetes: type 1 diabetes, type 2 diabetes and gestational diabetes. Roughly 415 million people had diabetes worldwide as of 2015; among those, $90 \%$ of the cases are type 2 diabetes [151]. With the improvement of life conditions, the incidence of diabetes increased rapidly. If diabetes cannot be well controlled, the function of some tissues will be disordered, which results in weight loss, fatigue, poor immunity and complications. These complications could bring great pain to patients and even endanger their lives. Hence, 
detection of diabetes in its early stages is critical for increasing the life quality of diabetic patients and has become an important topic in diabetes research.

With the worldwide prevalence of diabetes, the methods for monitoring glucose level in blood have received considerable interest. The conventional electrochemical method for measurement of glucose in blood is painful and has some limitations [152]. One promising and noninvasive technique for detection of diabetes is the analysis of VOCs in exhaled breath, which has been used as a tool for screening of many diseases [153-156]. Acetone level in exhaled breath correlated very well with glucose level in blood of diabetic patients [157]. Acetone is produced by lipolysis, absorbed into the bloodstream, and exhaled in the breath. There is not enough functioning insulin to help deliver glucose to the diabetes patients' cells. In this case, their body burns fat for energy, which leads to large amount of acetone in blood and breath [158]. Acetone concentration was found to elevate by at least two orders of magnitude in the plasma of diabetic patients. Acetone in diabetic breath is higher than $1.71 \mathrm{ppm}$, while its concentration in healthy people is lower than 0.76 ppm $[18,159]$. Acetone has been regarded as an important biomarker of diabetes [160, 161]. Analysis of acetone in breath has been used as a supplementary tool for diagnosis of diabetes.

Different thiol functionalized AuNP films have been investigated as sensing materials for detecting acetone in ideal conditions (dry air) in Chapter 2 and 3 without accounting for the effect of water vapor, which is a major component of the human breath. In this Chapter, we study thiol functionalized AuNP sensors for detecting acetone in exhaled breath. Three gold-thiolate protected nanoparticles were prepared 
and cast on interdigitated electrodes, which are DDT, MTT and monoalkoxyurea thiol 1. Also, microreactors are used to measure acetone concentration in breath samples.

\section{Materials and Methods}

\subsection{Materials and Sampling}

Hydrogen tetrachloroaurate $\left(\mathrm{HAuCl}_{4}\right)$, 1-dodecanethiol, 4-methoxy- $\alpha$ toluenethiol, tetraoctylammonium bromide (TOAB), acetone (>99.9\%), DNPH (>99\%) and $\mathrm{HClO}_{4}(70$ wt.\%) were purchased from Sigma Aldrich. Monoalkoxyurea thiol 1 is as-synthesized in Dr Nantz's group (see Chapter 3 for detail). Sodium borohydride was purchased from Fluka. Acetonitrile (HPLC grade) was obtained from $\mathrm{BDH}$. DNPH was purified by crystallization twice in acetonitrile before use. Other reagents were used without prior purification. Tedlar bags were purchased from Supelco (Bellefonte, PA). Cylinder air was obtained from Welders Supply Co. of Louisville. Deionized water was used throughout the experiment.

Exhaled breath (1 L) was collected in Tedlar bags from healthy subjects after overnight fasting. The collection of exhaled breath samples was approved by the internal review board (IRB). Prior to use, the new Tedlar bags were cleaned three times by using dry air. Dry air sample was from cylinder air. In order to test the effect of humidity, we injected different amounts of water into $1 \mathrm{~L}$ Tedlar bags to achieve the desired relative humidity $(\mathrm{RH})$. Theoretically, $1 \% \mathrm{RH}$ is equivalent to $312 \mathrm{ppm}$ water at $25{ }^{\circ} \mathrm{C}$. For example, to make $20 \% \mathrm{RH}$, we injected $23 \mu \mathrm{L}$ of water inside $1 \mathrm{~L}$ 
Tedlar bag until all the water evaporated. The same procedure was carried out for $40 \%, 60 \%, 80 \%$ and $90 \%$ RH. However, the water molecules start to aggregate at high humidity, which can also be seen in exhaled breath samples, and the response of the sensor may saturate at high humidity.

The synthesis of AuNP functionalized with 4-methoxy- $\alpha$-toluenethiol and 1dodecanethiol is described in Chapter II and AuNPs with monoalkoxyurea thiol 1 is described in Chapter III. Thiol structures are listed in Table 7.

Table 7 Thiol used for breath analysis

\begin{tabular}{cccc}
\hline Thiol & Symbol & Structure \\
4-methoxy- $\alpha$-toluenethiol & MTT & DDT & \\
1-dodecanethiol & Urea thiol 1 \\
thiol &
\end{tabular}




\subsection{Microreactor Preparation}

Microreactor is used to calculate the concentration of acetone in breath samples. The design, simulation, and fabrication of microreactor were reported in Dr. M. Li's dissertation [162]. The optical picture and SEM picture of a fabricated microreactor is shown in Figure 4.1. The microreactor was prepared a day before the breath samples were analyzed. A constant amount of $30 \mu \mathrm{L}$ acidified DNPH Solution (DNPH: $0.1 \mathrm{~mol} / \mathrm{L}, \mathrm{HClO}_{4}: 0.8 \mathrm{wt} \%$, acetonitrile $(\mathrm{ACN})$ as solvent) was impregnated into the microreactor and coated on walls of microreactor. Then, ACN was evaporated under vacuum at $50{ }^{\circ} \mathrm{C}$ by keeping the microreactor in the vacuum oven for 1 hour. After evaporation, fused silica tubes with $350 \mu \mathrm{m}$ o.d. and $250 \mu \mathrm{m}$ i.d. were connected to the inlet and outlet ports of the microreactors with an epoxy-based bonding agent. Two ends were sealed by parafilm and stored in the refrigerator before use. 


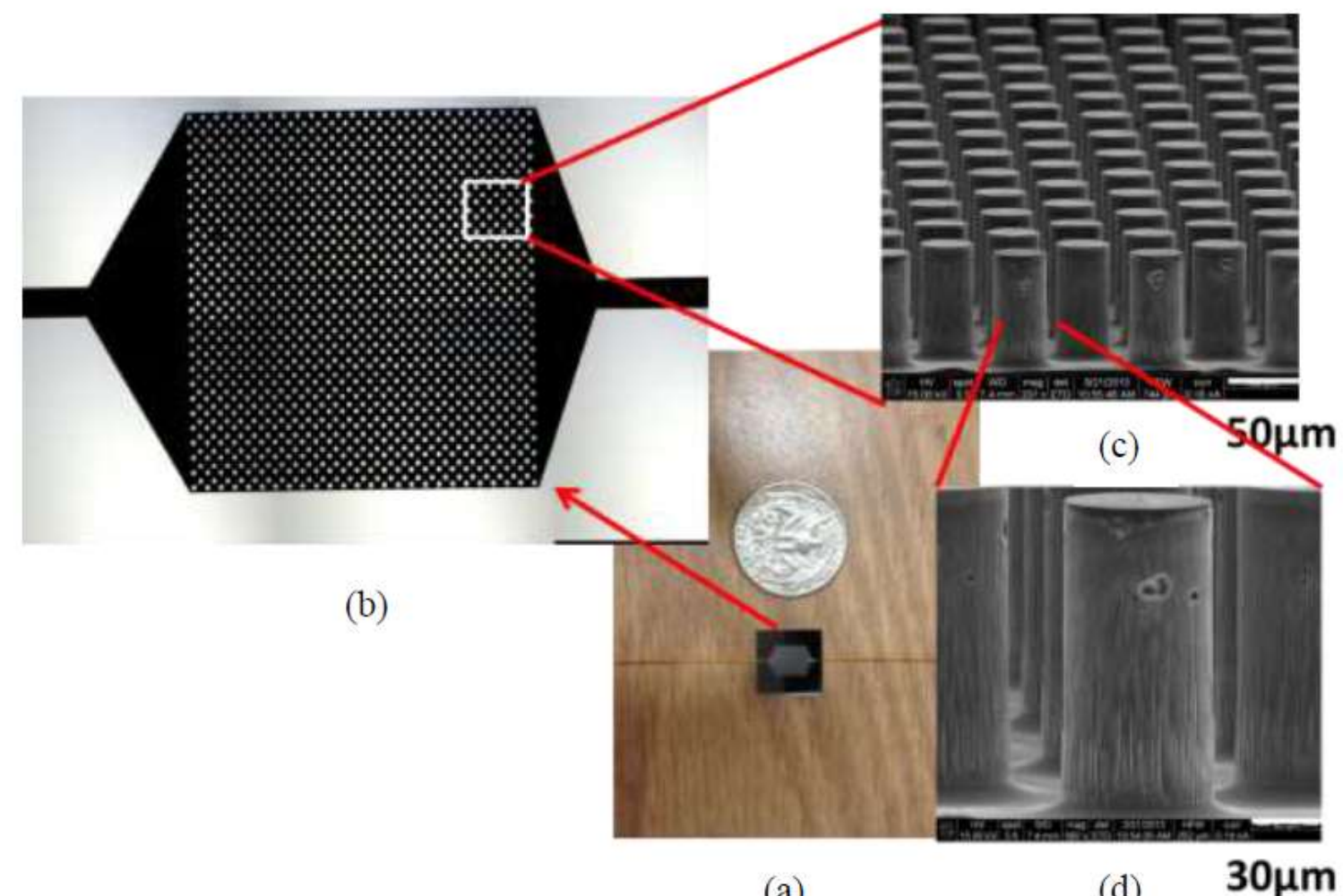

(a)

(d)

Figure 4.1 Optical and SEM graphs of one microreactor. (a) Schematic illustration of one microreactor compared with a quarter coin, (b) Optical micrograph of a fabricated microreactor with thousands of internal micropillars, (c) SEM micrographs of the micropillar arrays, (d) SEM micrographs of one micropillar. (Reprinted from [162]: M. Li, 2013).

\subsection{Evacuation of Exhaled Breath Samples Through Microreactors}

Healthy human breath samples $(1 \mathrm{~L})$ were collected by $1 \mathrm{~L}$ Tedlar bags after overnight fasting and after lunch and kept at room temperature to determine the initial acetone concentration in breath by microreactor, which was fabricated in the 
Nanotechnology Center at University of Louisville. The exhaled breath was then transferred to the lab within 3 hours after sample collections. The microreactor and sample bag were connected in the evacuation system shown in Figure 4.2. Five samples can be analyzed at a time by connecting five microreactors to the system in parallel. A mechanical needle valve made by Hoke Co. was used to adjust the flow rate of the sample flow through the microreactor. The flow rate of each line was adjusted to $3.5 \mathrm{~mL} / \mathrm{min}$ (measured by Agilent Technologies gas flowmeter, model \# 5067-0223) before the evacuation process. Then each sample bag was completely evacuated. The whole process was timed, so the exact volume of each sample bag can be calculated. After complete evacuation, the process would continue for an additional 30 minutes to make sure that the semi volatile compounds adsorbed on the sidewall of the bags were evacuated. The whole sampling process lasts about 5 hours.

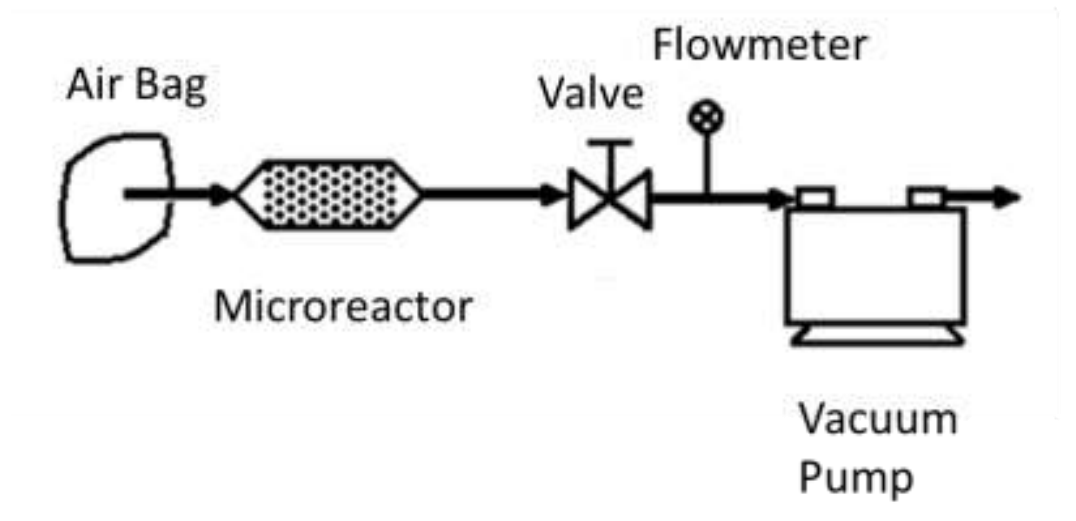

Figure 4.2 Schematic flow diagram of the evacuation setup 


\subsection{Analysis of Captured Carbonyls}

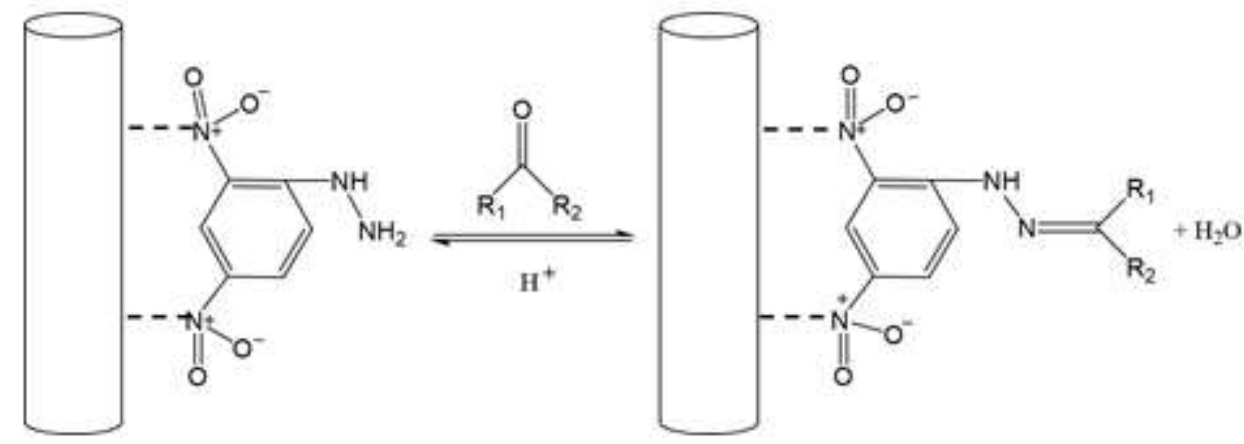

Figure 4.3 The reaction of DNPH with carbonyl compounds

During evacuation, carbonyls were reacted with DNPH to form hydrazone products (Figure 4.3). After evacuation, the microreactor was disconnected from the system. The formed DNPH-carbonyl products and unreacted DNPH are eluted by 150 $\mu \mathrm{L}$ ACN from one slightly pressurized vial through the microreactor and then into an empty collecting sample vial (Figure 4.4). The eluted solution samples were then analyzed by HPLC system.

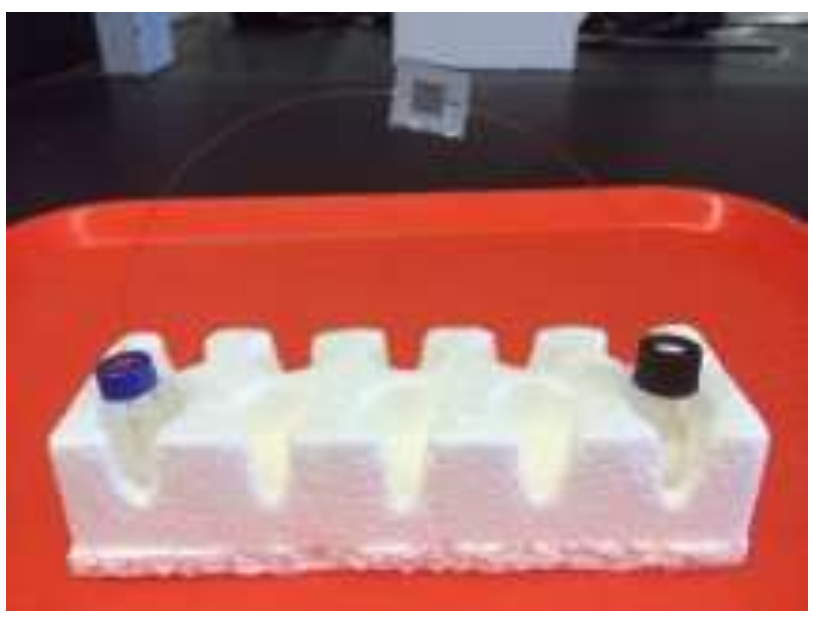

Figure 4.4 Acetonitrile elution of the microreactors. 
The Agilent 1100 HPLC system was used with a Restek Ultra C18 150mm $\times$ $4.6 \mathrm{~mm} 5 \mu \mathrm{m}$ column. The injection volume was $10 \mu \mathrm{L}$ and quantification was carried out at $365 \mathrm{~nm}$. The binary mobile phase gradient was prepared. Eluent A was prepared to contain water- acetonitrile- tetrahydrofuran (THF)- iso- propanol (59:30:10:1, V/V), Eluent B consisted of acetonitrile and water $(65: 35, \mathrm{~V} / \mathrm{V})$. The gradient program was a series of linear gradients from initial condition of $100 \%$ A to $60 \% \mathrm{~A}$ at $10 \mathrm{mins}$, then to $40 \%$ at $17 \mathrm{mins}$ and $20 \% \mathrm{~A}$ at $20 \mathrm{mins}$, then to $100 \% \mathrm{~A}$ at 24 mins with a total run time of $40 \mathrm{~min}$. Calibration curve for DNPH-acetone was determined by analyzing a series of standard DNPH-acetone solutions and regressing the peak-area response on concentration.

\section{Results and Discussion}

\subsection{Humidity Effect on the Thiol Functionalized Gold Nanoparticle-Coated Chemiresistor Sensor}

Thiol functionalized gold nanoparticle sensor is a promising candidate for detection of organic vapors, or even diagnosis of diseases, from exhaled breath because of its wide detection range, the possibility to operate at room temperature, low power consumption, and simple operating principle. However, it is also known that the sensing properties of many types of sensor are affected by the humidity at lower operating temperature. There have been many investigations about the effect of humidity on sensors and about the improvement of sensor properties against the 
humidity [49, 163-166]. The gold nanoparticle sensor is also required to respond stably against the various environmental conditions and the effect of humidity is another very important component that should be considered.

Here, the responses to different humidities are investigated on different thiol functionalized AuNP sensors. The relative humidity $(\mathrm{RH})$ in human breath is normally larger than $90 \%$. Figure $3.10 \mathrm{~B}$ in Chapter III shows monoalkoxyurea thiol 1 sensor's response to $\mathrm{RH}$ less than $5 \%$, which is always well below that of $1 \mathrm{ppb}$ acetone. Therefore, the sensor works quite well under low RH. Furthermore, we studied the response to water vapor for $\mathrm{H}_{2} \mathrm{O}$ concentrations from dry air to $20,40,60$, 80 and $90 \%$ RH on different sensors with dry air as blank (Figure 4.5). As seen, sensor responses to water vapor continue increasing with $\mathrm{RH}$ increasing from 20 to 90\%. As for water, whose relative permittivity is much higher than the medium material permittivity, the partitioning of water into the film can result in a significant change in the medium dielectric properties. The effect of water partitioning on the medium electric properties becomes more significant at higher humidity while water molecules begin to aggregate and the desorption process is slow. The aggregation of water molecules could completely change the film electric properties while ion current exists. Monoalkoxyurea thiol 1 sensor response changes the most with RH increasing, while 1-dodecanethiol sensor response changes the least. This is because there's a higher hydrogen bonding interaction between urea thiol and water molecules, so the sensor will respond higher to humidity. 


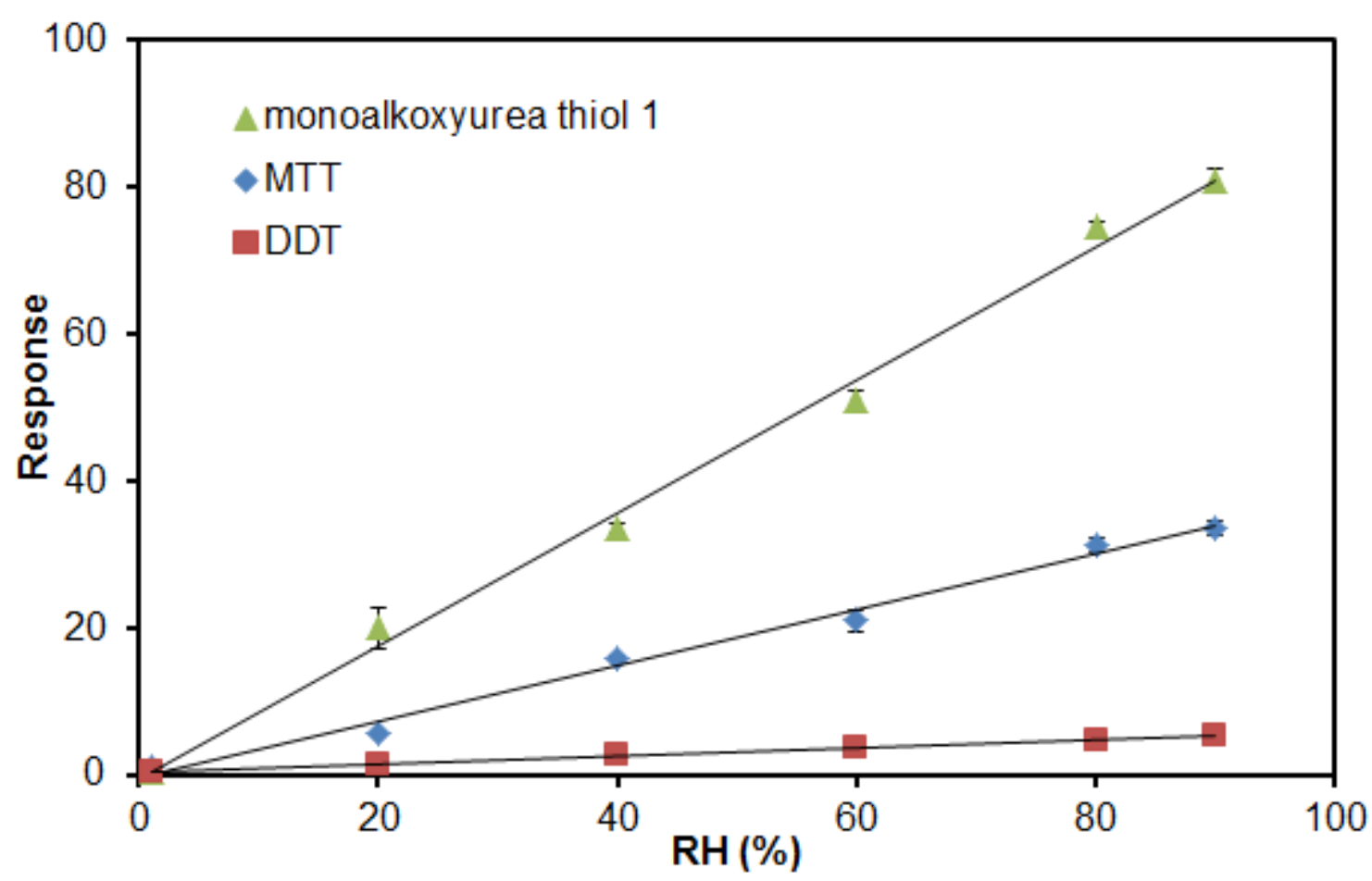

Figure 4.5 Sensor responses to water vapor at indicated relative humidity.

\subsection{Breath Sample Storage Time}

In order to collect breath samples of diabetic patients and transport them to the laboratory, it is important to know how long and what temperature the samples can be stored in the Tedlar bags. Analyte loss might occur due to adsorption, degradation or diffusion through the Tedlar bag walls although Tedlar bags are commonly used for collecting environmental air and breath sample. Here, we compared the MTT sensor response of the breath samples stored at $40{ }^{\circ} \mathrm{C}$ and room temperature for six different times (1, 2, 3, 4, 5 and 6 hours) after breath collection. Figure 4.6 and Figure 4.7 show that the samples are more stable at room temperature and there is almost no response change in 4 hours, while the samples are only stable for less than 2 hours at 
$40{ }^{\circ} \mathrm{C}$. In the following measurements, the breath sample was finished testing in 4 hours.

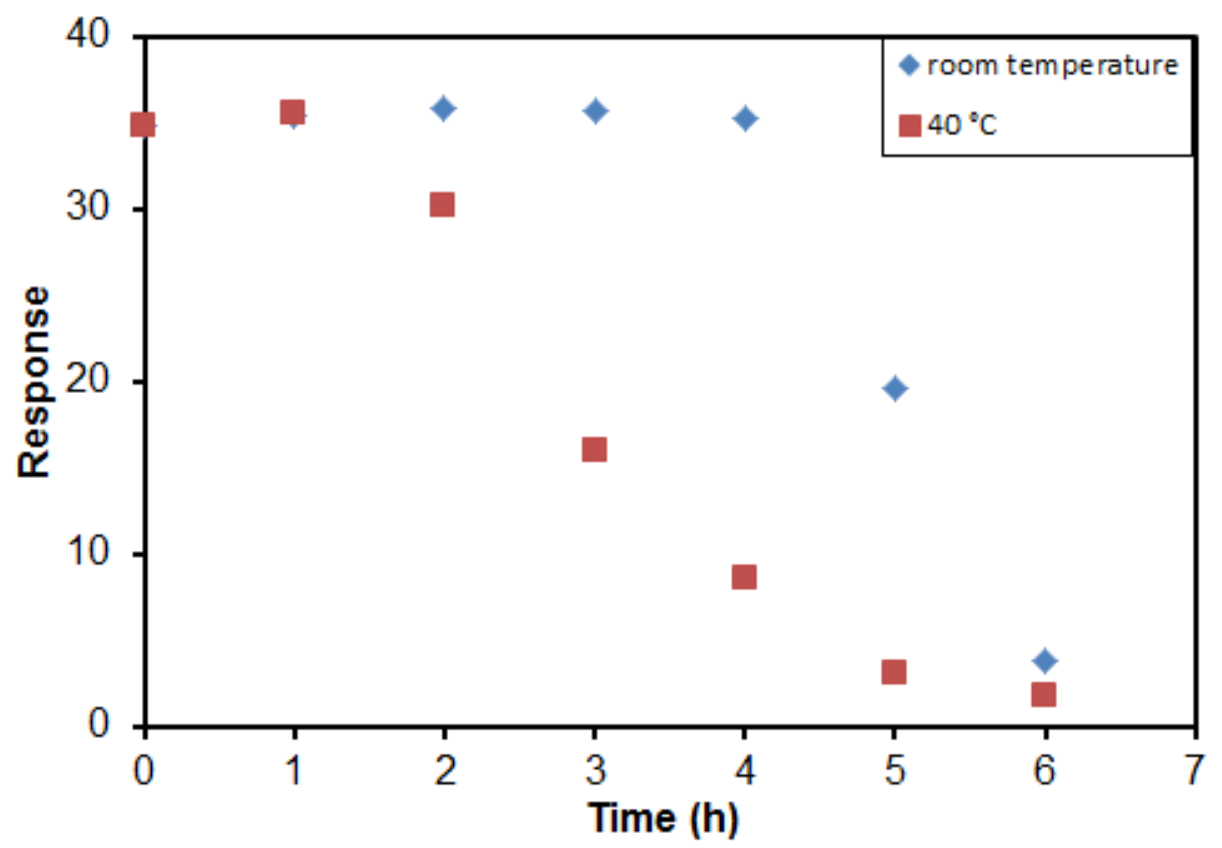

Figure 4.6 Stability of breath sample at $40^{\circ} \mathrm{C}$ and room temperature for MTT sensor

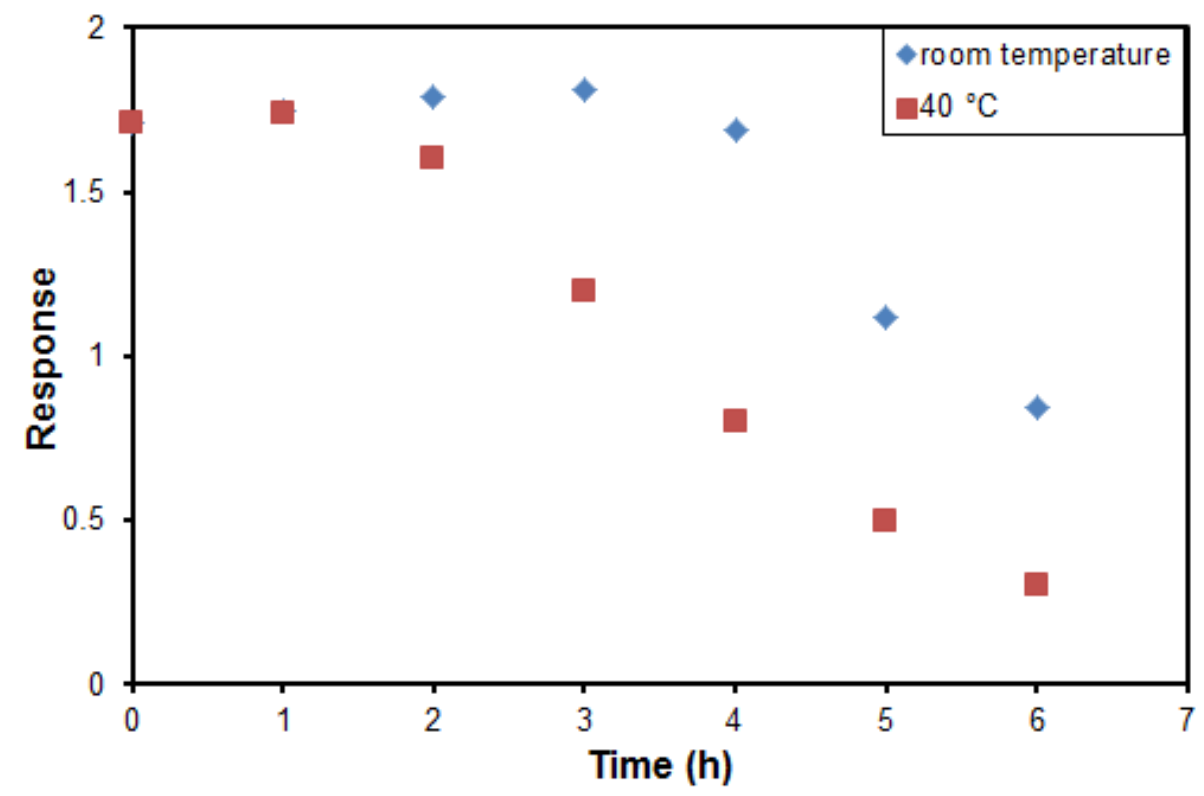

Figure 4.7 Stability of breath sample at $40{ }^{\circ} \mathrm{C}$ and room temperature for DDT sensor 


\subsection{Determination of Acetone Concentration in Breath Samples by Microreactor}

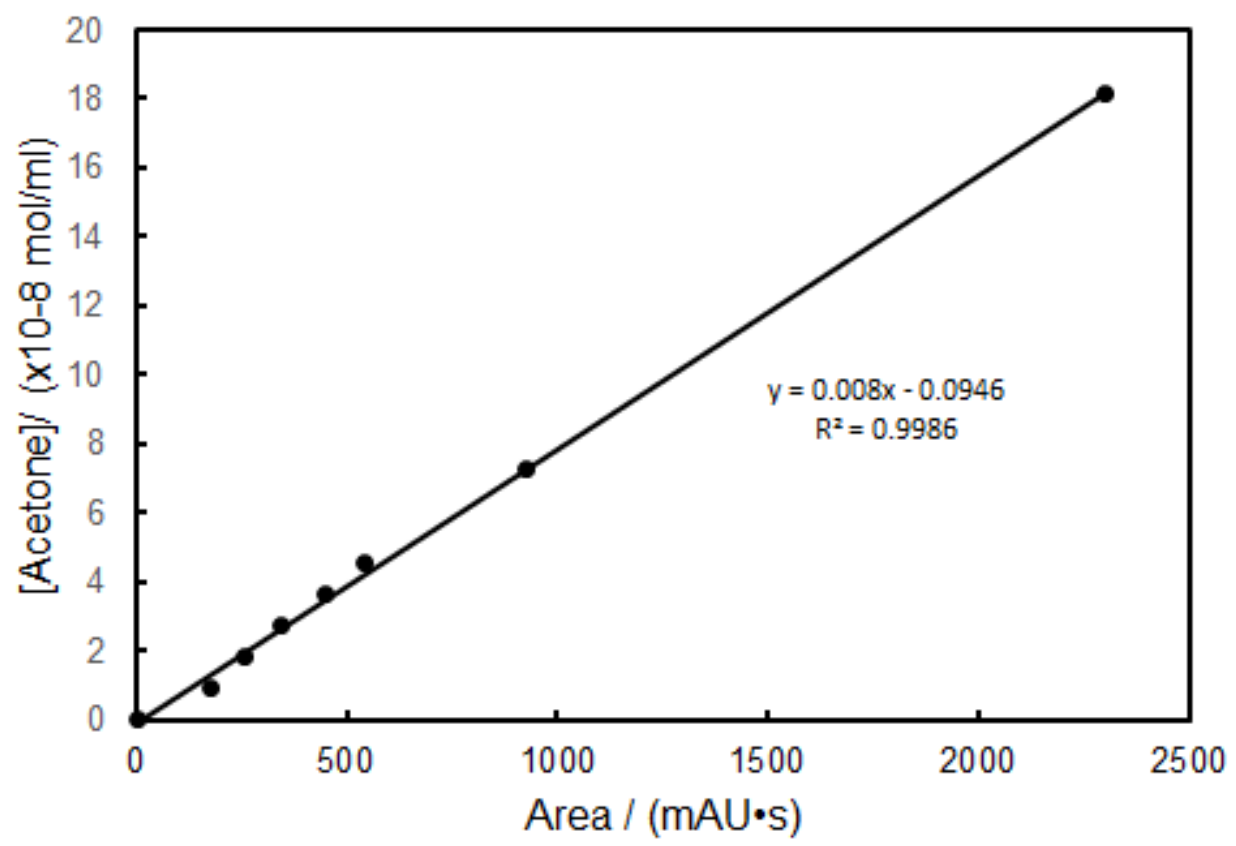

Figure 4.8 Calibration curve for DNPH-acetone concentration vs peak area

The calibration curves (Figure 4.8) show linear relationship over a DNPHacetone concentration range of 0.5 to $200 \mu \mathrm{mol} / \mathrm{mL}$, with correlation coefficients of over 0.99 . From the calibration curve, the amount of acetone can be calculated by the area of the peak. For example, Figure 4.9 shows a HPLC spectrum of one breath sample. The retention time of acetone is at $14.86 \mathrm{~min}$. The area of the peak at 14.86 min can be obtained from the spectrum, which is $1978.9 \mathrm{mAU} \cdot \mathrm{S}$. So the amount of acetone in the exhaled breath sample in $0.15 \mathrm{ml} \mathrm{ACN}$ elution can be calculated as follows:

$$
0.008 * 1978.9 * 10^{-8} * 0.15=2.37 * 10^{-8} \mathrm{~mol}=23.7 \mathrm{nmol}=0.53 \mathrm{ppm}
$$




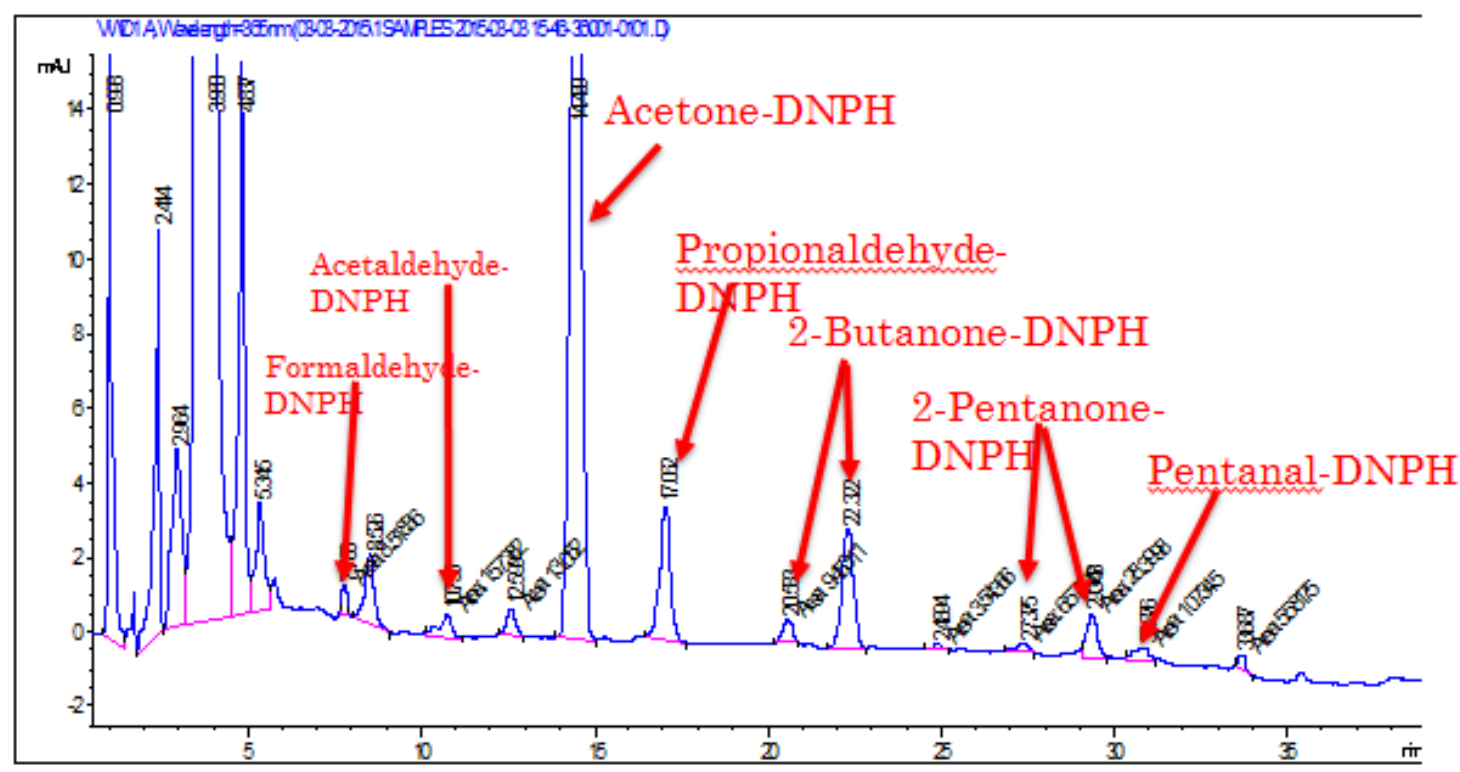

Figure 4.9 HPLC spectrum of one exhaled breath sample

The results shown in Table 8 indicate that acetone concentration in healthy people's breath sample is between $0.25 \mathrm{ppm}$ and $0.67 \mathrm{ppm}$, which is consistent with data in literature $[18,156,167]$. Here we use the average concentration of $0.4 \mathrm{ppm}$ as the breath acetone concentration. 
Table 8 Summary of acetone concentration in breath samples for different volunteers at different times.

\begin{tabular}{|c|c|c|c|}
\hline Volunteer & Age & Time & Acetone concentration \\
\hline \multirow{4}{*}{1} & & Morning 1 & $0.56 \mathrm{ppm}$ \\
\cline { 3 - 4 } & \multirow{3}{*}{27} & Morning 2 & $0.29 \mathrm{ppm}$ \\
\cline { 3 - 4 } & & Morning 3 & $0.45 \mathrm{ppm}$ \\
\cline { 3 - 4 } & & After lunch 1 & $0.28 \mathrm{ppm}$ \\
\cline { 3 - 4 } & & After lunch 2 & $0.43 \mathrm{ppm}$ \\
\cline { 3 - 4 } & After lunch 3 & $0.42 \mathrm{ppm}$ \\
\hline \multirow{3}{*}{2} & \multirow{3}{*}{29} & After lunch 1 & $0.53 \mathrm{ppm}$ \\
\cline { 3 - 4 } & & After lunch 2 & $0.35 \mathrm{ppm}$ \\
\cline { 3 - 4 } & & After lunch 3 & $0.43 \mathrm{ppm}$ \\
\hline \multirow{3}{*}{3} & \multirow{3}{*}{25} & After lunch 1 & $0.42 \mathrm{ppm}$ \\
\cline { 3 - 4 } & & After lunch 2 & $0.25 \mathrm{ppm}$ \\
\cline { 3 - 4 } & & After lunch 3 & $0.35 \mathrm{ppm}$ \\
\hline \multirow{3}{*}{4} & \multirow{3}{*}{30} & After lunch 1 & $0.27 \mathrm{ppm}$ \\
\cline { 3 - 4 } & & After lunch 2 & $0.31 \mathrm{ppm}$ \\
\cline { 3 - 4 } & After lunch 3 & $0.29 \mathrm{ppm}$ \\
\hline \multirow{3}{*}{5} & \multirow{3}{*}{35} & After lunch 1 & $0.67 \mathrm{ppm}$ \\
\cline { 3 - 4 } & & After lunch 2 & $0.62 \mathrm{ppm}$ \\
\cline { 3 - 4 } & & After lunch 3 & $0.51 \mathrm{ppm}$ \\
\hline
\end{tabular}

\subsection{Breath Sample Analysis}

Different amounts of acetone $(0.1,0.2,0.5,1,2,3 \mathrm{ppm})$ were spiked into breath samples of healthy people to simulate acetone levels of diabetic patients. The initial concentration of acetone is $0.4 \mathrm{ppm}$ in the breath sample. Figure 4.10 to 4.12 show three sensors' responses to acetone vapor in breath samples at different concentrations. The response increased linearly according to the concentration of acetone. Every sensor of the array undergoes a rapid and fully reversible change in 
electrical resistance when exposed to the breath sample. From the three figures, we can see the ability of the sensors to detect acetone in breath samples even though there's very high relative humidity in breath. The background of all the samples prepared for each sensor is with the same relative humidity and contains the same amount of other compounds. With the increasing of acetone concentration, the response increases. The response value in breath is different with that in dry air, because breath sample has so many compounds and also humidity plays an important role. 

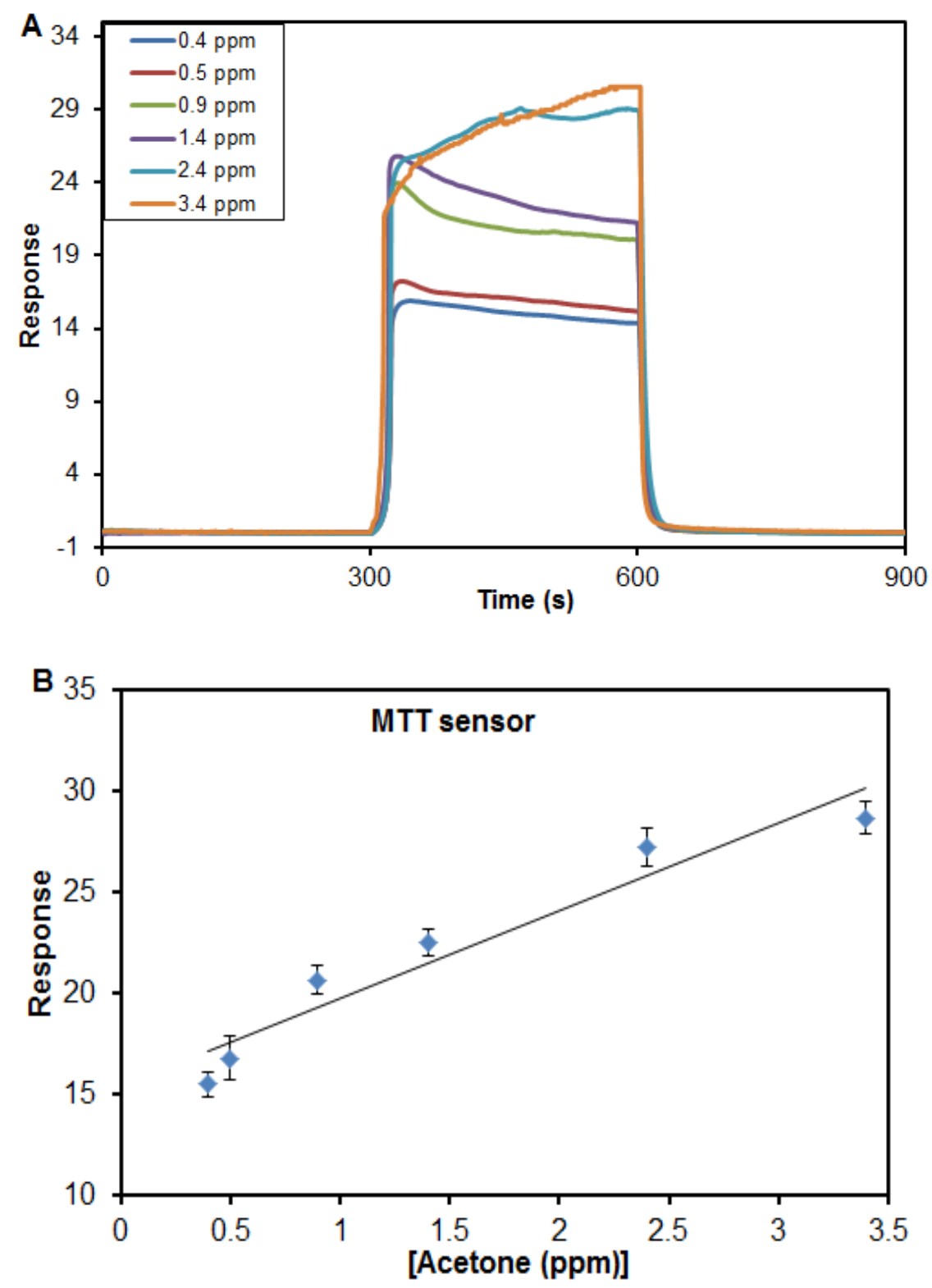

Figure 4.10 (A) Responses of MTT AuNP sensor to breath sample with different concentrations $(0.1,0.5,1,2,3 \mathrm{ppm})$ of spiked acetone; (B) MTT AuNP sensor response versus acetone concentration in breath sample. 

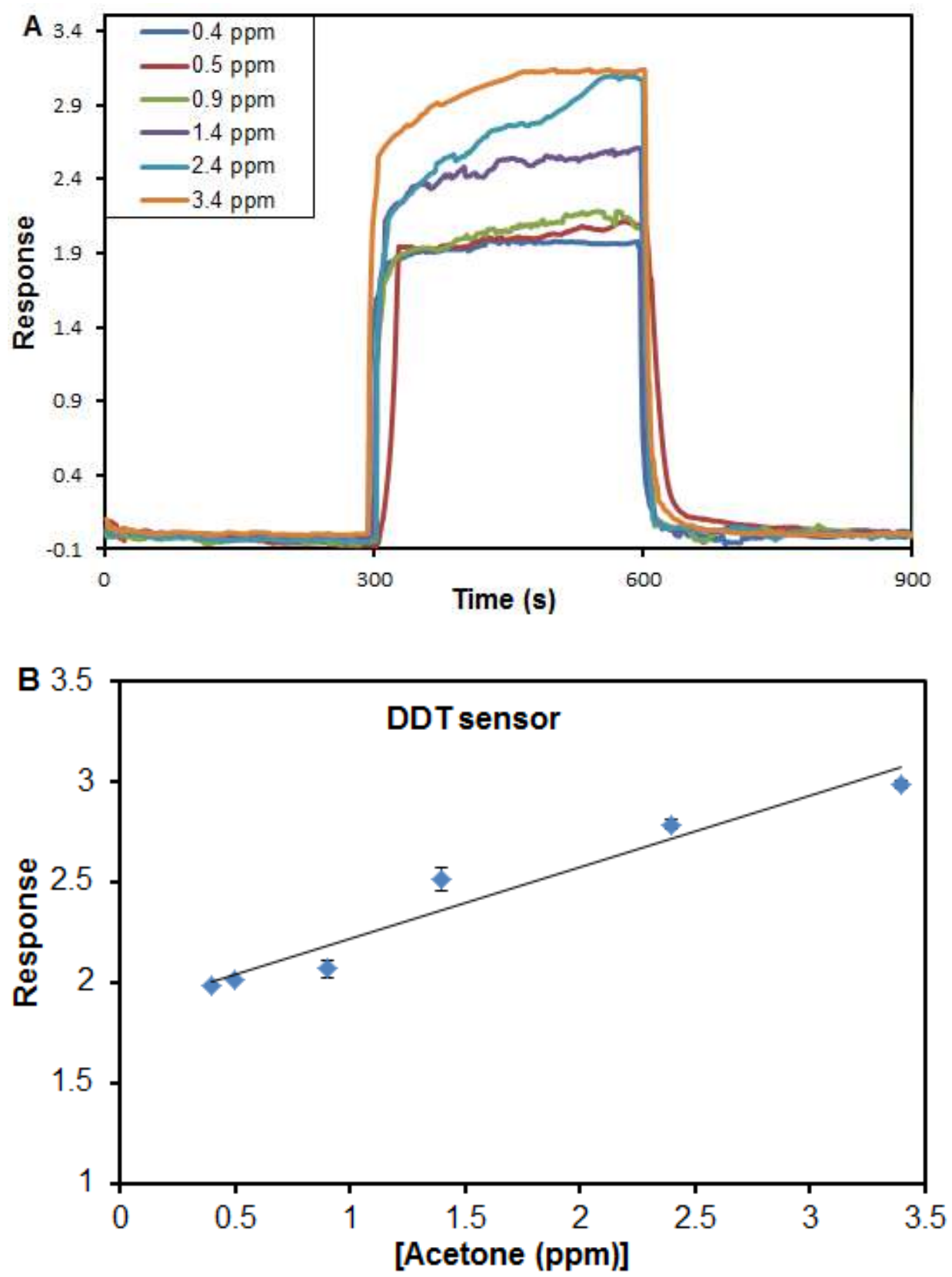

Figure 4.11 (A) Responses of DDT AuNP sensor to breath sample with different concentrations $(0.1,0.5,1,2,3 \mathrm{ppm})$ of spiked acetone; (B) DDT AuNP sensor response versus acetone concentration in breath sample. 

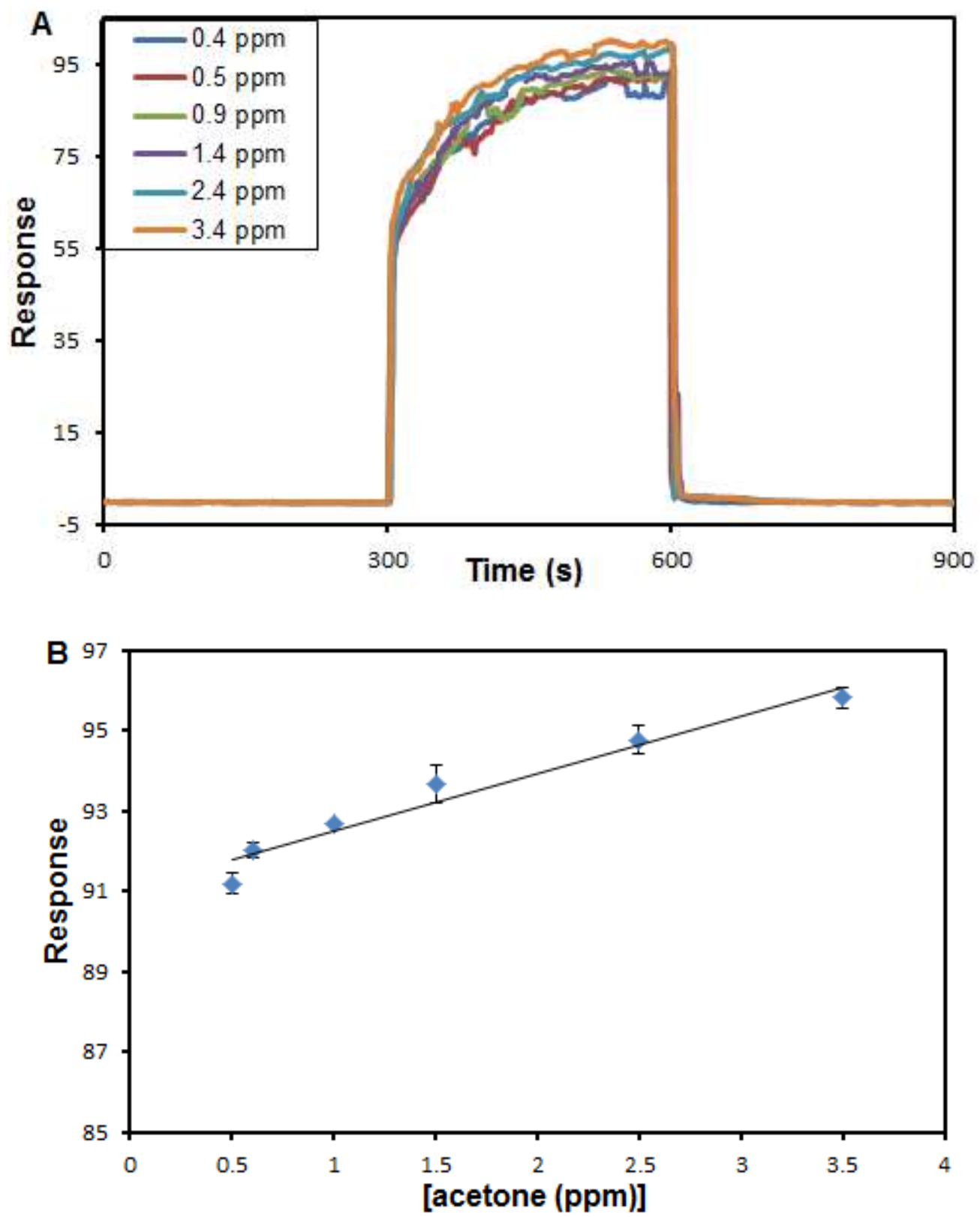

Figure 4.12 (A) Responses of monoalkoxyurea thiol 1 AuNP sensor to breath sample with different concentrations $(0.1,0.5,1,2,3 \mathrm{ppm})$ of spiked acetone; (B) urea thiol 1 AuNP sensor response versus acetone concentration in breath sample. 


\section{Conclusion}

Hyspler et al. demonstrated that it is feasible to use Tedlar bags to collect exhaled breath and applied SPME to analyze for isoprene [168]. However, Tedlar bags have two main disadvantages: (1) Analyte loss due to adsorption, degradation or diffusion through bag walls. (2) Residual acetone and other impurities derived from the manufacturing process. We found when storage time was more than 4 hours at room temperature, analyte loss occurred, so that short storage times could avoid sample loss. In our study, after collection of exhaled breath by using Tedlar bags, the breath sample was analyzed in $30 \mathrm{~min}$. To get rid of any residual acetone, new Tedlar bags were cleaned three times by using cylinder air prior to use. The prepared bags were analyzed by microreactor. We found that no acetone was detected in the prepared Tedlar bags, which means that the residual acetone was removed by displacement with dry air. Therefore, Tedlar bags can be used to collect human breath.

In this Chapter, we have demonstrated that an electronic nose based on functionalized gold nanoparticles can distinguish different levels of acetone in breath samples. Increasing RH to $90 \%$ increased the response of the three sensors, which reduced the sensor's sensitivity to acetone. However, at the same background of RH, our sensors were still able to tell different concentrations of acetone, which shows great promise for fast, easy and cost-effective diagnosis of diabetes. DDT sensor is better to be used under high humidity because dodecanethiol is hydrophobic and humidity does not have much influence to the response. Monoalkoxyurea thiol sensor shows higher sensitivity and it shows better response when adsorbents are used to 
remove moisture. The devices are inexpensive, portable and amenable to use, making them potentially valuable in diagnosing diabetes in early stages. 


\section{CHAPTER V}

\section{IMPROVING SENSITIVITY OF GOLD NANOPARTICLE SENSOR TOWARD ACETONE BY DEVISING DIFFERENT FUNCTIONAL MOTIFS}

\section{Introduction}

According to the results from Chapter 3, we found that tert-butyl monoalkoxyurea thiol-derived sensor is very sensitive to acetone. The detection range is very wide from $0.1 \mathrm{ppb}$ to $1000 \mathrm{ppm}$. The results are encouraging and prompted us to pursue the plan outlined for the synthesis of series 2 and series 3 motifs presented in Table 9 to increase sensitivity and selectivity for sensing acetone. These thiols also can interact with carbonyl compounds via hydrogen bonds.

A hydrogen bond is the electrostatic attraction between two polar groups that occurs when a hydrogen $(\mathrm{H})$ atom covalently bonded to a highly electronegative atom such as nitrogen $(\mathrm{N})$, oxygen $(\mathrm{O})$ or fluorine $(\mathrm{F})$ experiences the electrostatic field of another highly electronegative atom nearby.

Our synthetic efforts focus on developing thiol-based substrates fitted with binding elements for carbonyl analytes. As the respective sensors are developed, extrapolation to develop motifs for detecting acetone will be pursued. A key to 
achieving high sensitivities as well as functional group selectivity, possibly even single compound selectivity, will be to secure synthetic approaches that provide rapid access to numerous analogs to address not only these challenges but also issues surrounding gold nanoparticle formulation and nanosensor engineering. Consequently, we developed highly flexible synthetic routes to prepare carbonyl-binding substrates (Table 9). Our selection of binding motifs for these series follows from observations made by multiple groups in studies on catalysis via molecular recognition [169]. For example, thiourea-NH or urea-NH-carbonyl interactions are well documented [170, 171] as significant molecular binding motifs. We will augment these interactions by preparing the more acidic alkoxyureas and bis-alkoxyureas and examine the influences on sensor response.

Table 9 Three series of urea thiol synthesized to functionalize gold nanoparticles
Series 1
Series 2
Series 3
Monoalkoxyurea thiol
Alkylurea thiol
Bis-alkoxyurea thiol<smiles>CC(C)(C)NC(=O)NOCCCCCS</smiles><smiles>CC(C)(C)CNC(=O)NC(C)(C)C</smiles><smiles>CC(C)(C)ONC(=O)NOCCCCS</smiles><smiles>CCCCONC(=O)NC1CCCCC1</smiles><smiles>CC(C)CNC(=O)NC1CCCCC1</smiles><smiles>O=C(NOCCCCS)NOC1CCCCC1</smiles> 
<smiles>O=C(NOCCCCCS)Nc1ccccc1</smiles><smiles>CCCNC(=O)Nc1ccccc1</smiles><smiles>CC(CS)CONC(=O)Nc1ccc(F)cc1</smiles><smiles>O=C(NCCCS)Nc1ccc(F)cc1</smiles>

\section{Experimental}

Three series of thiols were prepared by Dr. Mandapati Raju (University of Louisville, Department of Chemistry).

\subsection{Procedures for Synthesis of Monoalkoxyurea Thiols}
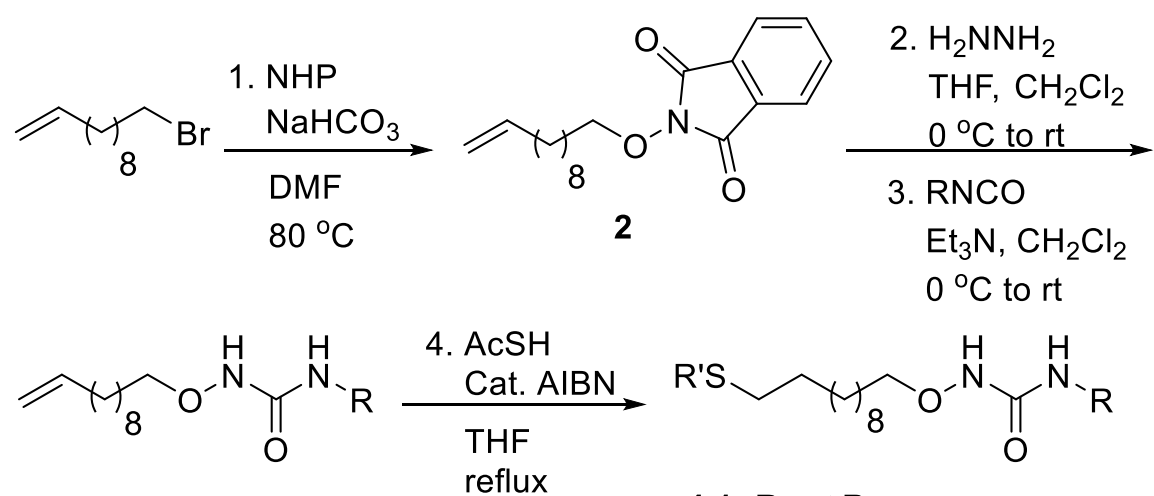

3.1: $R=t-B u$ reflux<smiles>[R5]CCCCCONC(=O)N[R]</smiles>

3.2: $\mathrm{R}=\mathrm{C}_{6} \mathrm{H}_{11}$

3.3: $\mathrm{R}=\mathrm{C}_{6} \mathrm{H}_{5}$

3.4: $\mathrm{R}=4-\mathrm{F}-\mathrm{C}_{6} \mathrm{H}_{4}$
4.1: $R=t-B u$
4.2: $\mathrm{R}=\mathrm{C}_{6} \mathrm{H}_{11}$ 4.3: $\mathrm{R}=\mathrm{C}_{6} \mathrm{H}_{5}$
5. $\mathrm{HCl}$ 4: $\mathbf{R}^{\prime}=\mathrm{CH}_{3} \mathrm{C}(\mathrm{O})$ 4.4: $\mathrm{R}=4-\mathrm{F}-\mathrm{C}_{6} \mathrm{H}_{4}$ reflux

Figure 5.1 Synthetic routes for preparation of monoalkoxyurea thiols 4.1-4.4. 
The general hydrazinolysis and urea formation procedure is the same with tert-butyl monoalkoxyurea thiol, which is described in detail in Chapter 3. The difference is as follows:

1-(tert-butyl)-3-(undec-10-en-1-yloxy)urea (3.1): Using the general procedure outlined above, $2(1.00 \mathrm{~g}, 3.17 \mathrm{mmol})$ was transformed into $3.10 .62 \mathrm{~g}$ (68\%) as a colorless liquid; TLC, $\mathrm{R}_{f}=0.51$ (2.5:7.5, EtOAc:hexane).

1-cyclohexyl-3-(undec-10-en-1-yloxy)urea (3.2): Using the general procedure outlined above, $2(0.51 \mathrm{~g}, 1.61 \mathrm{mmol})$ was transformed into $3.20 .36 \mathrm{~g}$ (71\%) as a white solid; TLC, $\mathrm{R}_{f}=0.44$ (2.5:7.5, EtOAc:hexane).

1-phenyl-3-(undec-10-en-1-yloxy)urea (3.3): Using the general procedure outlined above, 2 (0.30 g, $0.95 \mathrm{mmol})$ was transformed into $3.30 .22 \mathrm{~g}(77 \%)$ as a white solid; TLC, $\mathrm{R}_{f}=0.65$ (2.5:7.5, EtOAc:hexane).

1-(4-fluorophenyl)-3-(undec-10-en-1-yloxy)urea (3.4): Using the general procedure outlined above, $2(0.52 \mathrm{~g}, 0.47 \mathrm{mmol})$ was transformed into $3.40 .35 \mathrm{~g}$ $(66 \%)$ as a white solid.

\section{General thioacetate formation procedure:}

A solution of urea $3.1(0.50 \mathrm{~g}, 1.75 \mathrm{mmol})$ and azobis(isobutyronitrile) (0.043 $\mathrm{g}, 0.26 \mathrm{mmol})$ in dry THF $(20 \mathrm{~mL})$ was heated to reflux whereupon thioacetic acid $(0.25 \mathrm{~mL}, 3.51 \mathrm{mmol})$ was added via syringe. The reaction mixture was stirred at reflux $8 \mathrm{~h}$ and then concentrated by rotary evaporation. The residue was purified by $\mathrm{SiO}_{2}$ column chromatography, eluting with a 15:85 mixture of EtOAc:hexane, to afford thioacetate derivative. 
$S$-(11-((3-(tert-Butyl)ureido)oxy)undecyl) ethanethioate (4.1): Using the general procedure outlined above, $3.1(0.50 \mathrm{~g}, 1.75 \mathrm{mmol})$ was transformed into thioester $4.10 .48 \mathrm{~g}(75 \%)$ as a pale yellow liquid.

S-(11-((3-cyclohexylureido)oxy)undecyl) ethanethioate (4.2): Using the general procedure outlined above, $3.2(0.25 \mathrm{~g}, 0.80 \mathrm{mmol})$ was transformed into thioester $4.20 .26 \mathrm{~g}(83 \%)$ as a pale white solid.

S-(11-((3-phenylureido)oxy)undecyl) ethanethioate (4.3): Using the general procedure outlined above, $3.3(0.20 \mathrm{~g}, 0.66 \mathrm{mmol})$ was transformed into thioester $\mathbf{4 . 3}$ $0.26 \mathrm{~g}(83 \%)$ as a white solid.

S-(11-((3-(4-fluorophenyl)ureido)oxy)undecyl) ethanethioate (4.4): Using the general procedure outlined above, $3.4(0.25 \mathrm{~g}, 0.28 \mathrm{mmol})$ was transformed into thioester 4.4 $0.18 \mathrm{~g}(58 \%)$ as a pale yellow solid.

\section{General thioaceatate cleavage procedure:}

To a solution of thioester urea $4(0.50 \mathrm{~g}, 1.38 \mathrm{mmol})$ in EtOH $(10 \mathrm{~mL})$ was added $12 \mathrm{~N} \mathrm{HCl}(1.1 \mathrm{~mL}, 11.0 \mathrm{mmol})$. The reaction mixture was heated to reflux and stirred. After $4 \mathrm{~h}$, the reaction mixture was cooled and then concentrated by rotary evaporation. The residue was dissolved in water $(10 \mathrm{~mL})$. The resultant solution was cooled to $0{ }^{\circ} \mathrm{C}$ and the $\mathrm{pH}$ was adjusted to slightly alkaline using saturated $\mathrm{NaHCO}_{3}$ solution. The aqueous layer then was extracted with $\mathrm{CH}_{2} \mathrm{Cl}_{2}(4 \mathrm{X} 30 \mathrm{~mL})$. The combined organic phase was washed with saturated $\mathrm{NaCl}(2 \times 20 \mathrm{~mL})$, dried $\left(\mathrm{MgSO}_{4}\right)$, filtered and concentrated under reduced pressure to afford thiol urea. 
1-(tert-Butyl)-3-((11-mercaptoundecyl)oxy)urea (1.1). Using the general procedure outlined above, $4.1(0.50 \mathrm{~g}, 1.38 \mathrm{mmol})$ was transformed into thiol urea $\mathbf{1 . 1}$ $(0.38 \mathrm{~g}, 86 \%)$ as a pale yellow liquid that was directly applied to gold nanoparticles.

1-cyclohexyl-3-((11-mercaptoundecyl)oxy)urea (1.2): Using the general procedure outlined above, $4.2(0.230 \mathrm{~g}, 0.57 \mathrm{mmol})$ was transformed into thiol urea $1.2(0.18 \mathrm{~g}, 88 \%)$ as a white solid that was directly applied to gold nanoparticles.

1-((11-mercaptoundecyl)oxy)-3-phenylurea (1.3): Using the general procedure outlined above, $4.3(0.175 \mathrm{~g}, 0.46 \mathrm{mmol})$ was transformed into thiol urea $1.3(0.12 \mathrm{~g}, 77 \%)$ as a white solid that was directly applied to gold nanoparticles.

1-(4-fluorophenyl)-3-((11-mercaptoundecyl)oxy)urea (1.4): Using the general procedure outlined above, $4.4(0.155 \mathrm{~g}, 1.17 \mathrm{mmol})$ was transformed into thiol urea $1.4(0.110 \mathrm{~g}, 79 \%)$ as a pale yellow solid that was directly applied to gold nanoparticles. 


\subsection{Procedures for Synthesis of Alkylurea Thiols}
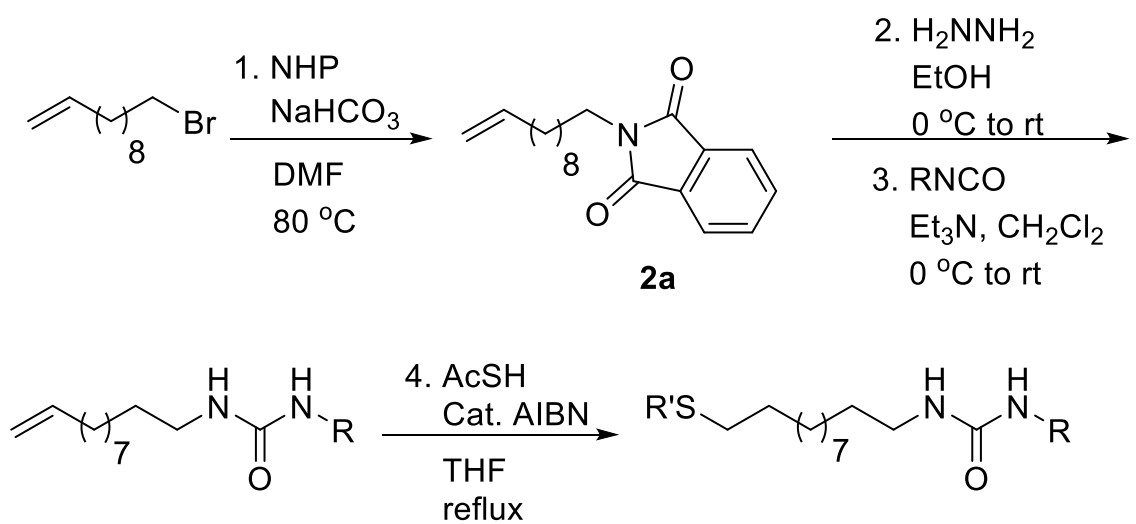<smiles>[R5]CC[Y4]([H])([H])CCNC(=O)N[R]</smiles>

3.1a: $R=t-B u$

4.1 $\mathrm{a}: \mathrm{R}=\boldsymbol{t}-\mathrm{Bu}$

3.2a: $R=\mathrm{C}_{6} \mathrm{H}_{11}$

3.3a: $\mathrm{R}=\mathrm{C}_{6} \mathrm{H}_{5}$

3.4a: $\mathrm{R}=4-\mathrm{F}-\mathrm{C}_{6} \mathrm{H}_{4}$

4.2a: $R=\mathrm{C}_{6} \mathrm{H}_{11}$

4.3a: $\mathrm{R}=\mathrm{C}_{6} \mathrm{H}_{5}$

4.4a: $\mathrm{R}=4-\mathrm{F}-\mathrm{C}_{6} \mathrm{H}_{4} \quad$ reflux

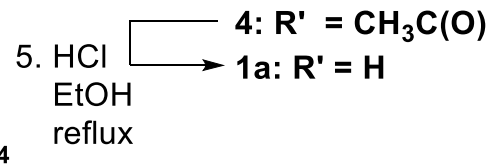

Figure 5.2 Synthetic routes for preparation of alkylurea thiols 4.1a-4.4a.

2-(undec-10-en-1-yl)isoindoline-1,3-dione (2a). 11-bromo-1-undecene (2.82 $\mathrm{mL}, 12.8 \mathrm{mmol})$ was dissolved in DMF $(60 \mathrm{~mL})$ at room temperature. Phthalimide potassium salt $(2.85 \mathrm{~g}, 15.43 \mathrm{mmol})$ was added to the mixture, and the reaction mixture was heated to $80{ }^{\circ} \mathrm{C}$. After $12 \mathrm{~h}$, the reaction mixture was allowed to cool to room temperature and then poured into $5 \mathrm{wt} \%$ aqueous $\mathrm{HCl}(100 \mathrm{~mL})$. The aqueous layer was extracted with $\mathrm{CHCl}_{3}(4 \mathrm{X} 50 \mathrm{~mL})$. The combined organic layer was dried through $\left(\mathrm{MgSO}_{4}\right)$, filtered, and then concentrated by rotary evaporation. The crude product was purified by $\mathrm{SiO}_{2}$ column chromatography, eluting with a 20:85 mixture of EtOAc:hexane, to afford $3.64 \mathrm{~g}(94 \%)$ of $\mathbf{2 a}$ as a white solid. TLC, $\mathrm{R}_{f}=0.67$ (2.5:7.5, EtOAc:hexane).

\section{General hydrazinolysis and urea formation procedures:}

To a solution of phthalimide $2 \mathbf{a}(1.00 \mathrm{~g}, 3.34 \mathrm{mmol})$ in $\mathrm{EtOH}(20 \mathrm{~mL})$ at $0{ }^{\circ} \mathrm{C}$ was added hydrazine $(0.4 \mathrm{~mL}, 8.35 \mathrm{mmol})$. The reaction mixture was stirred at room 
temperature for $12 \mathrm{~h}$ and then resulted phthalylhydrazide salts were filtered. The retentate was concentrated by rotary evaporation. The crude amine product so obtained was used directly in the next step without further purification

To a stirred solution of the crude amine $(0.56 \mathrm{~g}, 3.32 \mathrm{mmol})$ in dry $\mathrm{CH}_{2} \mathrm{Cl}_{2}(15$ $\mathrm{mL})$ at room temperature under argon was added dry $\mathrm{Et}_{3} \mathrm{~N}(0.64 \mathrm{~mL}, 4.53 \mathrm{mmol})$ via syringe. The reaction mixture was cooled to $0{ }^{\circ} \mathrm{C}$ and then $t$-butylisocyanate $(0.34$ $\mathrm{mL}, 3.02 \mathrm{mmol}$ ) was added dropwise over $5 \mathrm{~min}$. The reaction mixture was slowly allowed to reach room temperature and stirred $10 \mathrm{~h}$ before concentrating by rotary evaporation. The residue was purified by $\mathrm{SiO}_{2}$ flash column chromatography, eluting with a 1:4 mixture of EtOAc:hexane, to afford desired urea derivative.

1-(tert-butyl)-3-(undec-10-en-1-yl)urea (3.1a): Using the general procedure outlined above, 2a (1.00 g, $3.34 \mathrm{mmol})$ was transformed into 3.1a $0.65 \mathrm{~g}(72 \%)$ as a colorless liquid.

1-cyclohexyl-3-(undec-10-en-1-yl)urea (3.2a): Using the general procedure outlined above, 2a (0.79 g, $2.64 \mathrm{mmol})$ was transformed into 3.2a $0.58 \mathrm{~g}(75 \%)$ as a white solid.

1-phenyl-3-(undec-10-en-1-yl)urea (3.3a): Using the general procedure outlined above, 2a $(0.56 \mathrm{~g}, 1.87 \mathrm{mmol})$ was transformed into 3.3a $0.31 \mathrm{~g}(57 \%)$ as a white solid.

1-(4-flourophenyl)-3-(undec-10-en-1-yl)urea (3.4a): Using the general procedure outlined above, 2a $(0.49 \mathrm{~g}, 1.63 \mathrm{mmol})$ was transformed into $\mathbf{3 . 4 a} 0.32 \mathrm{~g}$ (63\%) as a pale yellow solid.

\section{General thioacetate formation procedure:}


A solution of urea 3.1a $(0.51 \mathrm{~g}, 1.90 \mathrm{mmol})$ and azobis(isobutyronitrile) $(0.047 \mathrm{~g}, 0.28 \mathrm{mmol})$ in dry THF $(20 \mathrm{~mL})$ was heated to reflux whereupon thioacetic acid $(0.25 \mathrm{~mL}, 3.51 \mathrm{mmol})$ was added via syringe. The reaction mixture was stirred at reflux $8 \mathrm{~h}$ and then concentrated by rotary evaporation. The residue was purified by $\mathrm{SiO}_{2}$ column chromatography, eluting with a 15:85 mixture of EtOAc:hexane, to afford thioacetate derivative.

$S$-(11-(3-(tert-butyl)ureido)undecyl) ethanethioate (4.1a): Using the general procedure outlined above, 3.1a $(0.51 \mathrm{~g}, 1.90 \mathrm{mmol})$ was transformed into thioester 4.1 $0.28 \mathrm{~g}(43 \%)$ as a pale yellow liquid.

S-(11-((3-cyclohexylureido)oxy)undecyl) ethanethioate (4.2a): Using the general procedure outlined above, 3.2a $(0.49 \mathrm{~g}, 1.66 \mathrm{mmol})$ was transformed into thioester 4.2a $0.26 \mathrm{~g}(42 \%)$ as a pale white solid.

S-(11-(3-phenylureido)undecyl) ethanethioate (4.3a): Using the general procedure outlined above, 3.3a $(0.26 \mathrm{~g}, 0.90 \mathrm{mmol})$ was transformed into thioester 4.3a $0.17 \mathrm{~g}(52 \%)$ as a white solid.

S-(11-(3-(4-flourophenyl)ureido)undecyl) ethanethioate (4.4a): Using the general procedure outlined above, 3.4a $(0.20 \mathrm{~g}, 0.65 \mathrm{mmol})$ was transformed into thioester $4.4 \mathrm{a} 0.11 \mathrm{~g}(44 \%)$ as a pale yellow solid.

\section{General thioaceatate cleavage procedure:}

To a solution of thioester urea $4(0.175 \mathrm{~g}, 0.50 \mathrm{mmol})$ in $\mathrm{EtOH}(5 \mathrm{~mL})$ was added $12 \mathrm{~N} \mathrm{HCl}(0.4 \mathrm{~mL}, 4.06 \mathrm{mmol})$. The reaction mixture was heated to reflux and stirred. After $4 \mathrm{~h}$, the reaction mixture was cooled and then concentrated by rotary evaporation. The residue was dissolved in water $(10 \mathrm{~mL})$. The resultant solution was 
cooled to $0{ }^{\circ} \mathrm{C}$ and the $\mathrm{pH}$ was adjusted to slightly alkaline using saturated $\mathrm{NaHCO}_{3}$ solution. The aqueous layer then was extracted with $\mathrm{CH}_{2} \mathrm{Cl}_{2}(4 \times 30 \mathrm{~mL})$. The combined organic phase was washed with saturated $\mathrm{NaCl}(2 \mathrm{X} 20 \mathrm{~mL})$, dried $\left(\mathrm{MgSO}_{4}\right)$, filtered and concentrated under reduced pressure to afford thiol urea.

1-(tert-butyl)-3-(11-mercaptoundecyl)urea (1.1a). Using the general procedure outlined above, $4.1 \mathrm{a}(0.175 \mathrm{~g}, 0.50 \mathrm{mmol})$ was transformed into thiol urea 1.1a $(0.115 \mathrm{~g}, 77 \%)$ as a pale yellow liquid that was directly applied to gold nanoparticles.

1-cyclohexyl-3-(11-mercaptoundecyl)urea (1.2a): Using the general procedure outlined above, $4.2 \mathrm{a}(0.191 \mathrm{~g}, 0.57 \mathrm{mmol})$ was transformed into thiol urea 1.2a $(0.15 \mathrm{~g}, 88 \%)$ as a white solid that was directly applied to gold nanoparticles.

1-(11-mercaptoundecyl)-3-phenylurea (1.3a): Using the general procedure outlined above, $\mathbf{4 . 3 a}(0.1 \mathrm{~g}, 0.27 \mathrm{mmol})$ was transformed into thiol urea $\mathbf{1 . 3 a}(0.08 \mathrm{~g}$, 89\%) as a white solid that was directly applied to gold nanoparticles.

1-(4-fluorophenyl)-3-(11-mercaptoundecyl)urea (1.4a): Using the general procedure outlined above, $4.4 \mathrm{a}(0.13 \mathrm{~g}, 0.34 \mathrm{mmol})$ was transformed into thiol urea 1.4a $(0.085 \mathrm{~g}, 74 \%)$ as a pale yellow solid that was directly applied to gold nanoparticles. 


\subsection{Procedure for Synthesis of bis-Alkoxyurea Thiols}

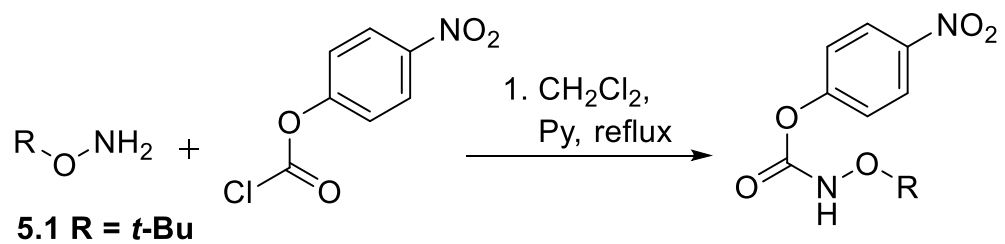

$5.2 \mathrm{R}=\mathrm{C}_{6} \mathrm{H}_{11}$<smiles>C=CCCON1C(=O)c2ccccc2C1=O</smiles>

2

2. $\mathrm{H}_{2} \mathrm{NNH}_{2}$

$6.1 \mathrm{R}=t-\mathrm{Bu}$

$6.2 \mathrm{R}=\mathrm{C}_{6} \mathrm{H}_{11}$

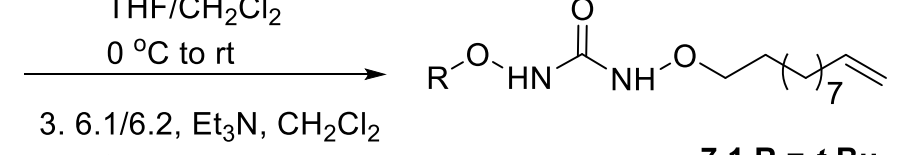
$0{ }^{\circ} \mathrm{C}$ to rt

$7.1 \mathrm{R}=\boldsymbol{t}-\mathrm{Bu}$

$7.2 \mathrm{R}=\mathrm{C}_{6} \mathrm{H}_{11}$

4. AcSH

Cat. AIBN

THF, reflux

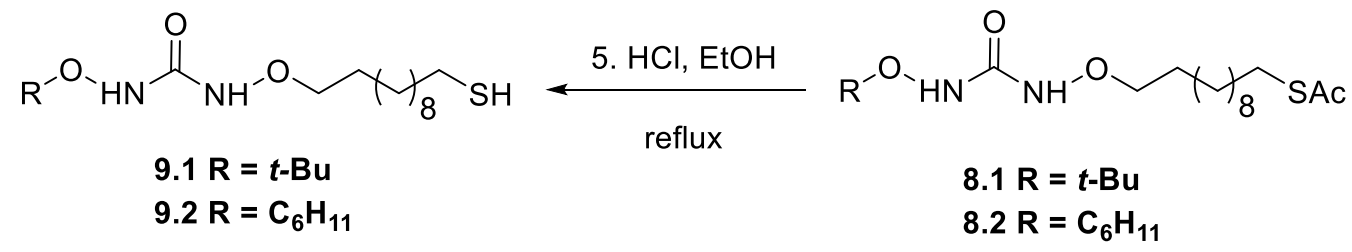

Figure 5.3 Synthetic routes for preparation of bis-alkoxy urea thiols 8.1-8.2.

\section{General procedure for aminooxy protection with 4-Nitrophenyl}

\section{chloroformate:}

O-(tert-butyl)hydroxylamine hydrochloride 5.1 (1.39 g, $11.11 \mathrm{mmol})$ was dissolved in methylene chloride $(100 \mathrm{~mL})$ followed by the addition of pyridine $(1.2$ $\mathrm{mL}, 13.89 \mathrm{mmol}$ ) under an argon atmosphere. The reaction flask was cooled to $0{ }^{\circ} \mathrm{C}$ and 4-nitrophenyl chloroformate $(2.24 \mathrm{~g}, 11.11 \mathrm{mmol})$ was added. Then the reaction mixture was heated at reflux for $24 \mathrm{~h}$. Upon cooling, the homogeneous organic solution was washed with $1 \mathrm{M} \mathrm{NaHCO}_{3}(3 \mathrm{X} 60 \mathrm{~mL})$ and $\mathrm{H}_{2} \mathrm{O}(2 \mathrm{X} 40 \mathrm{~mL})$. The 
organic fraction was dried with $\mathrm{MgSO}_{4}$, filtered, and concentrated. A chloroformhexane mixture $(5: 6,22 \mathrm{~mL})$ was added. The residue was purified by $\mathrm{SiO}_{2}$ column chromatography, eluting with a 20:80 mixture of EtOAc:hexane, to afford protected aminooxy derivative.

4-nitrophenyl tert-butoxycarbamate (6.1): Using the general procedure outlined above, $5.1(1.39 \mathrm{~g}, 11.11 \mathrm{mmol})$ was transformed into $6.11 .96 \mathrm{~g}(69 \%)$ as a white solid.

4-nitrophenyl (cyclohexyloxy)carbamate (6.2): Using the general procedure outlined above, $\mathbf{5 . 2}(1.13 \mathrm{~g}, 9.80 \mathrm{mmol})$ was transformed into $\mathbf{6 . 2} 1.39 \mathrm{~g}(50 \%)$ as a white solid.

\section{General hydrazinolysis and urea formation procedures:}

To a solution of phthalimide $2(0.8 \mathrm{~g}, 2.53 \mathrm{mmol})$ in $\mathrm{CH}_{2} \mathrm{Cl}_{2}(10 \mathrm{~mL})$ at $0{ }^{\circ} \mathrm{C}$ was added hydrazine $(8.2 \mathrm{~mL}$ of $1.0 \mathrm{M}$ solution in THF, $8.2 \mathrm{mmol})$. The reaction mixture was stirred at $0{ }^{\circ} \mathrm{C}$ for $1.5 \mathrm{~h}$ and then filtered. The retentate was washed with $\mathrm{CH}_{2} \mathrm{Cl}_{2}(10 \mathrm{~mL})$ and the combined organic solvents then were concentrated by rotary evaporation. The crude aminooxy product so obtained was used directly in the next step without further purification (Note: protect from carbonyl vapors, such as laboratory acetone).

To a stirred solution of the crude aminooxy $(0.45 \mathrm{~g}, 2.43 \mathrm{mmol})$ in dry $\mathrm{CH}_{2} \mathrm{Cl}_{2}$ $(20 \mathrm{~mL})$ at room temperature under argon was added $\operatorname{dry} \mathrm{Et}_{3} \mathrm{~N}(0.68 \mathrm{~mL}, 4.86 \mathrm{mmol})$ via syringe. The reaction mixture was cooled to $0{ }^{\circ} \mathrm{C}$ and 4-nitrophenyl tertbutoxycarbamate (6.1) (1.85 g, $7.29 \mathrm{mmol})$ was added. The reaction mixture was heated to reflux. After $8 \mathrm{~h}$ the reaction mixture was slowly allowed to reach room 
temperature and quenched with water $(50 \mathrm{~mL})$ and extracted with dichloromethane (3 x $100 \mathrm{~mL}$ ). The combined organic phase was dried over anhydrous $\mathrm{MgSO}_{4}$ and concentrated under reduced pressure. The crude product was via column chromatography (4:1 to $3: 2$, hexanes: ethyl acetate) to afford the product.

1-(tert-butyl)-3-(undec-10-en-1-yl)urea (7.1): Using the general procedure outlined above, $2(0.8 \mathrm{~g}, 2.53 \mathrm{mmol})$ was transformed into $7.10 .58 \mathrm{~g}(76 \%)$ as a pale yellow liquid.

1-(cyclohexyloxy)-3-(undec-10-en-1-yloxy)urea (7.2): Using the general procedure outlined above, $2(0.52 \mathrm{~g}, 1.64 \mathrm{mmol})$ was transformed into $7.20 .44 \mathrm{~g}$ $(83 \%)$ as a colorless liquid.

\section{General thioacetate formation procedure:}

A solution of urea $7.1(0.48 \mathrm{~g}, 1.59 \mathrm{mmol})$ and azobis(isobutyronitrile) $(0.04 \mathrm{~g}$, $0.24 \mathrm{mmol})$ in dry THF $(20 \mathrm{~mL})$ was heated to reflux whereupon thioacetic acid $(0.23$ $\mathrm{mL}, 3.19 \mathrm{mmol}$ ) was added via syringe. The reaction mixture was stirred at reflux 8 $\mathrm{h}$ and then concentrated by rotary evaporation. The residue was purified by $\mathrm{SiO}_{2}$ column chromatography, eluting with a 15:85 mixture of EtOAc:hexane, to afford thioacetate derivative.

\section{S-(2,2-dimethyl-5-oxo-3,7-dioxa-4,6-diazaoctadecan-18-yl) ethanethioate}

(8.1): Using the general procedure outlined above, $7.1(0.48 \mathrm{~g}, 1.59 \mathrm{mmol})$ was transformed into thioester $\mathbf{8 . 1} 0.45 \mathrm{~g}(75 \%)$ as a colorless liquid.

S-(11-((3-(cyclohexyloxy)ureido)oxy)undecyl) ethanethioate (8.2): Using the general procedure outlined above, $7.2(0.4 \mathrm{~g}, 1.22 \mathrm{mmol})$ was transformed into thioester 8.2 $0.36 \mathrm{~g}(73 \%)$ as a colorless liquid. 


\section{General thioaceatate cleavage procedure:}

To a solution of thioester urea $8.1(0.4 \mathrm{~g}, 1.06 \mathrm{mmol})$ in $\mathrm{EtOH}(5 \mathrm{~mL})$ was added $12 \mathrm{~N} \mathrm{HCl}(0.83 \mathrm{~mL}, 8.5 \mathrm{mmol})$. The reaction mixture was heated to reflux and stirred. After $4 \mathrm{~h}$, the reaction mixture was cooled and then concentrated by rotary evaporation. The residue was dissolved in water $(10 \mathrm{~mL})$. The resultant solution was cooled to $0{ }^{\circ} \mathrm{C}$ and the $\mathrm{pH}$ was adjusted to slightly alkaline using saturated $\mathrm{NaHCO}_{3}$ solution. The aqueous layer then was extracted with $\mathrm{CH}_{2} \mathrm{Cl}_{2}(4 \times 30 \mathrm{~mL})$. The combined organic phase was washed with saturated $\mathrm{NaCl}(2 \mathrm{X} 20 \mathrm{~mL})$, dried $\left(\mathrm{MgSO}_{4}\right)$, filtered and concentrated under reduced pressure to afford thiol urea.

1-(tert-butoxy)-3-((11-mercaptoundecyl)oxy)urea (9.1). Using the general procedure outlined above, $8.1(0.4 \mathrm{~g}, 1.06 \mathrm{mmol})$ was transformed into thiol urea $\mathbf{9 . 1}$ $(0.29 \mathrm{~g}, 81 \%)$ as a colorless gummy liquid that was directly applied to gold nanoparticles.

1-(cyclohexyloxy)-3-((11-mercaptoundecyl)oxy)urea (9.2): Using the general procedure outlined above, $\mathbf{8 . 2}(0.33 \mathrm{~g}, 0.82 \mathrm{mmol})$ was transformed into thiol urea $9.2(0.185 \mathrm{~g}, 62 \%)$ as a colorless gummy liquid. that was directly applied to gold nanoparticles.

\subsection{Synthesis of Gold Nanoparticles (AuNPs)}

The urea thiol functionalized AuNP synthesis procedure is the same as described in Chapter III for the monoalkoxyurea thiol 1. First, $0.05 \mathrm{~g}$ of $\mathrm{HAuCl}_{4}$ was dissolved in $4 \mathrm{~mL}$ of deionized water; $0.08 \mathrm{~g}$ of TOAB was dissolved in $20 \mathrm{~mL}$ of 
toluene. Then the aqueous $\mathrm{HAuCl}_{4}$ solution was added to the toluene TOAB solution with vigorous stirring until all the tetrachloroaurate transferred into the organic phase. Next, urea thiol (thiol: Au molar ratio of 1:1) was added into the above solution. An aqueous solution of $\mathrm{NaBH}_{4}$, freshly prepared by dissolving $0.056 \mathrm{~g} \mathrm{NaBH}_{4}$ in $4 \mathrm{~mL}$ deionized water, was slowly added with vigorous stirring to the toluene mixture. A rapid color change occurred as the $\mathrm{NaBH}_{4}$ solution was added. After stirring for at least 3 hours at room temperature, the black part was separated and dropwise added into $400 \mathrm{~mL}$ of ethanol with rapid stirring. After settling overnight at freezer, the mixture was centrifuged and washed with ethanol for 3 times and dried at $40{ }^{\circ} \mathrm{C}$ in an oven to yield thiol-coated AuNPs (ca.10 mg) suitable for sensor formation.

The thiol-coated AuNPs were dispersed in toluene $(0.2 \%)$ by sonication at room temperature for $20 \mathrm{~min}$, and then cast onto the IDE area by dropwise addition. The toluene was evaporated at room temperature to leave a flat, roughly circular film of thiol-coated gold nanoparticles. The thiol-coated AuNP sensors were then stabilized overnight at $40{ }^{\circ} \mathrm{C}$ in a vacuum oven.

\section{Sensor Response to Acetone}

Here, the response characteristics for different series of urea thiol functionalized AuNP sensors in response to acetone are described. There are 3 series of thiols that were used in this Chapter, including monoalkoxyurea thiols (Series 1), alkylurea thiols (Series 2) and bisalkoxyurea thiols (Series 3). There are 4 different functional group thiols in the first and second series, which are tert-butyl, cyclohexyl, 
phenyl, and $p$-fluorophenyl. Based on the results obtained from the first two series, we only included two different functional groups which are tert-butyl and cyclohexyl for the bisalkoxyurea thiols (Series 3).

\subsection{Response Results for Monoalkoxyurea Thiol Sensor Sensing Acetone}

First, we compare different functional groups of thiols in the same series. tertbutyl monoalkoxyurea thiol sensor's response to acetone is shown in Figure 3.10. Figure 5.4 to 5.6 show the response data to acetone vapor in air for cyclohexyl, phenyl and $p$-fluorophenyl monoalkoxyurea thiol sensors.

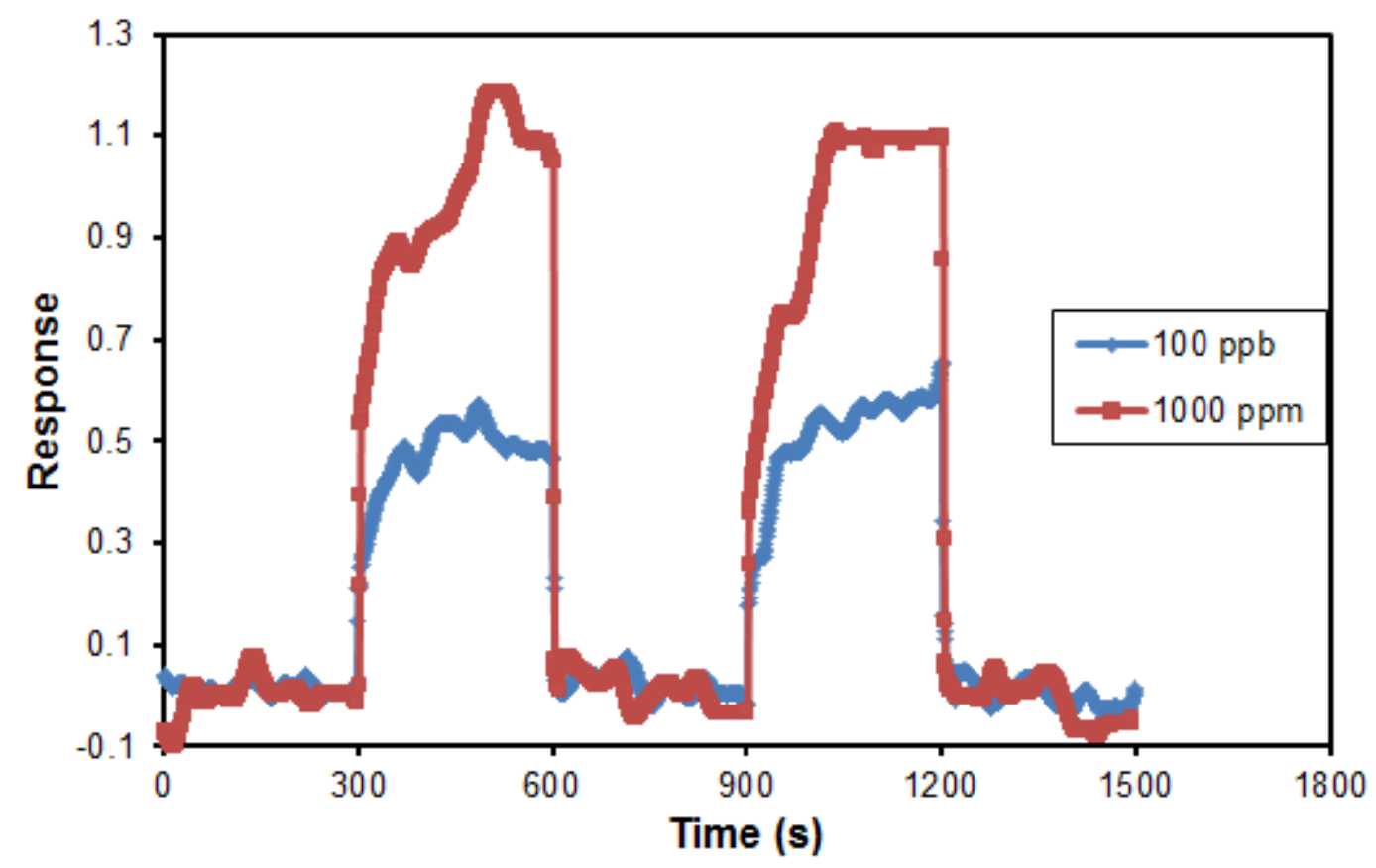

Figure 5.4 Cyclohexyl monoalkoxyurea thiol sensor response profile in response to $100 \mathrm{ppb}$ and $1000 \mathrm{ppm}$ of acetone vapor. 


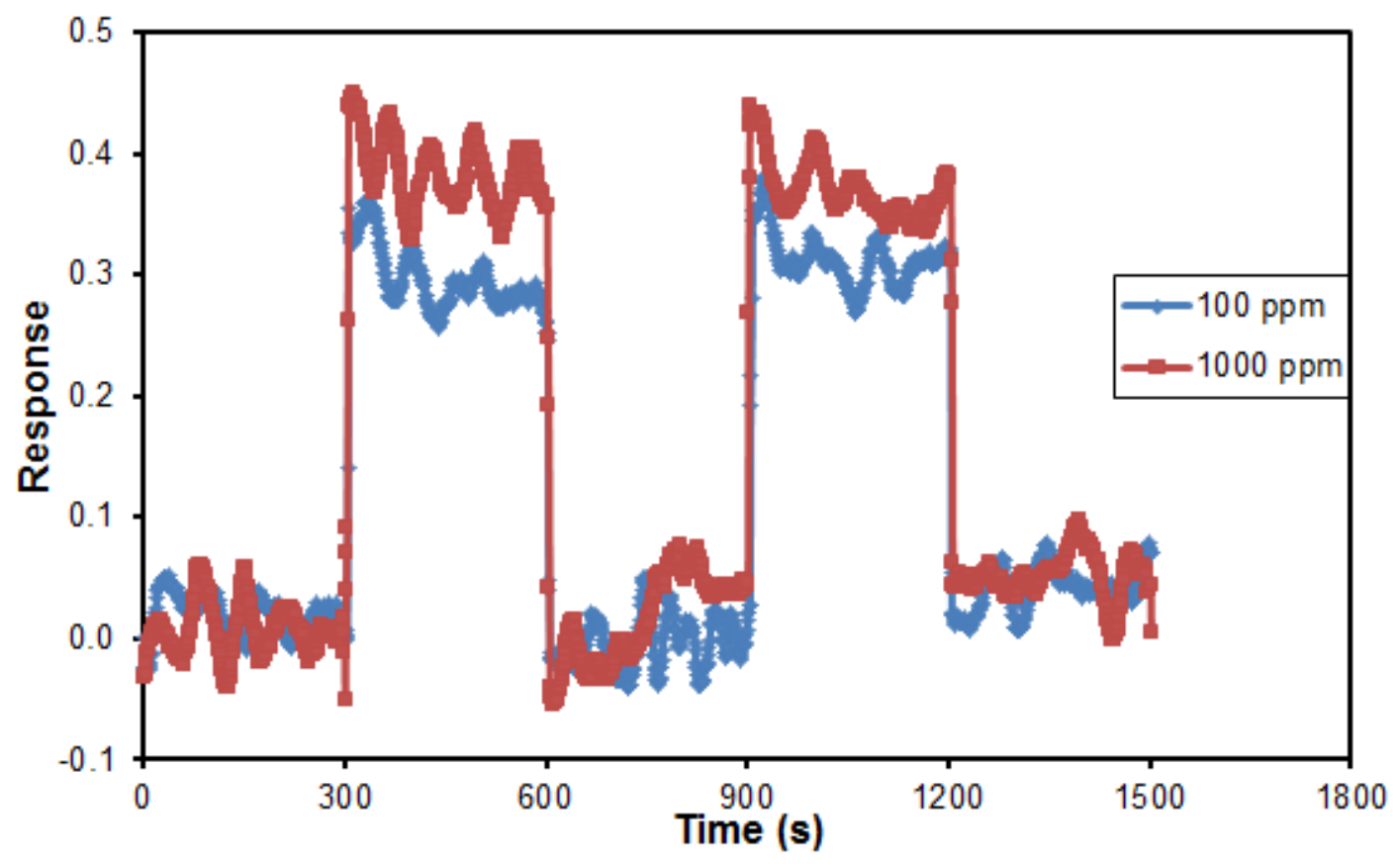

Figure 5.5 Phenyl monoalkoxyurea thiol sensor response profile in response to 100 ppm and $1000 \mathrm{ppm}$ of acetone vapor

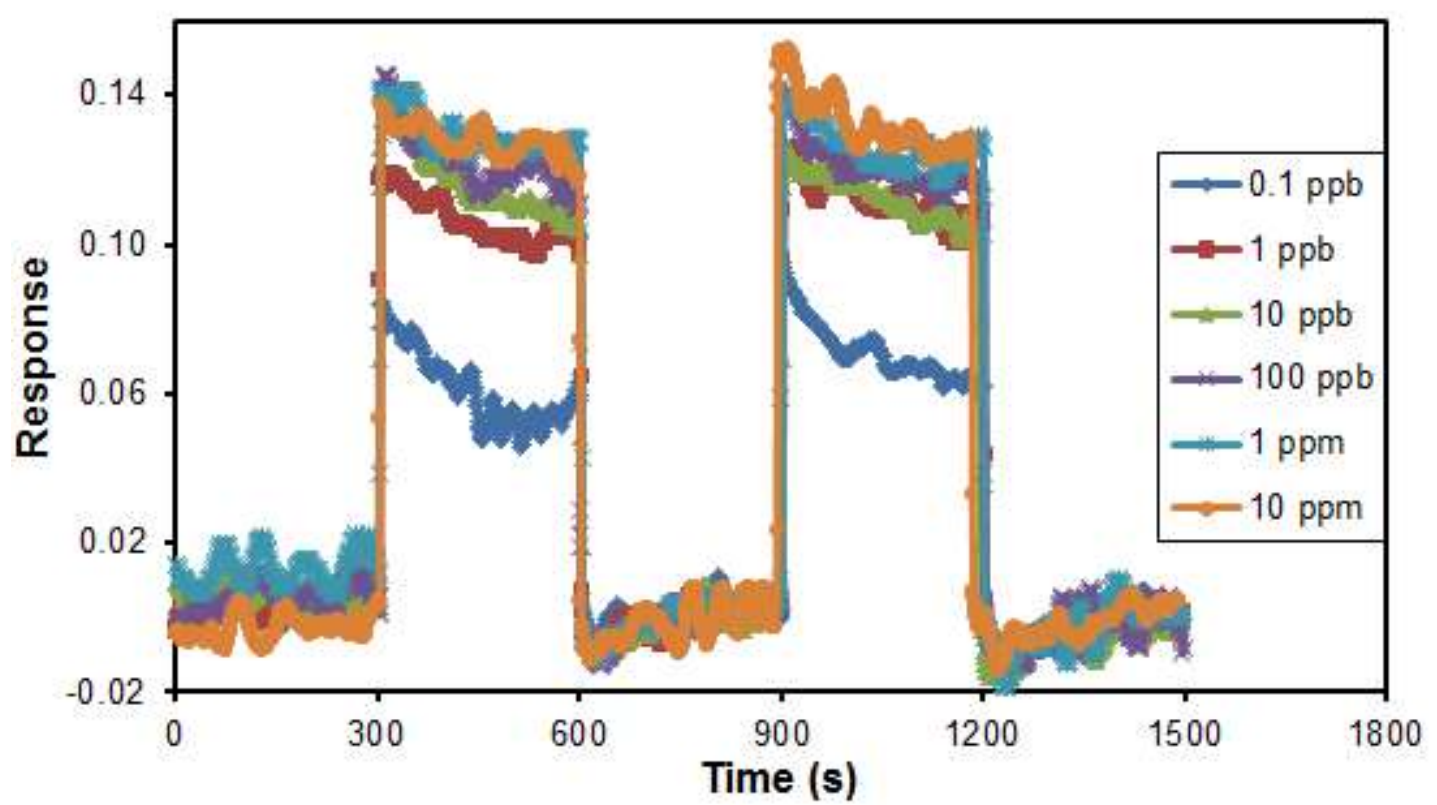

Figure $5.6 p$-fluorophenyl monoalkoxyurea thiol sensor response profile in response to $0.1 \mathrm{ppb}$ to $10 \mathrm{ppm}$ of acetone vapor. 
From Figure 3.10 and Figure 5.4-5.6, we can see that the tert-butyl monoalkoxyurea thiol sensor has the largest response and sensitivity to acetone vapor, and it shows the limit of detection of $0.1 \mathrm{ppb}$. However, for cyclohexyl and phenyl monoalkoxyurea thiol sensor, acetone vapor was not detected until the concentration was increased to higher than $100 \mathrm{ppb}$. The response and recovery time for these sensors are fast, usually in a few seconds. $p$-fluorophenyl monoalkoxyurea thiol sensor's response increased from 0.06 to 0.11 when acetone concentration increases from $0.1 \mathrm{ppb}$ to $1 \mathrm{ppb}$. Then the response stays almost the same when further increasing the concentration of acetone. The largest response observed for tert-butyl substituted monoalkoxyurea thiol sensor among other substituents in this series could be ascribed to steric effect as well its coupled electronic interactions. Thus, it is vital to control overall local network formation between urea moieties so as to maintain an optimal response with the carbonyl analyte. In this instance, the tert-butyl substituent is bulky enough to disrupt the network interactions in allowing access to carbonyl analyte.

Figure 5.7 shows the hydrogen bonding interaction between AuNP functionalized with tert-butyl monoalkoxyurea thiol and acetone. 


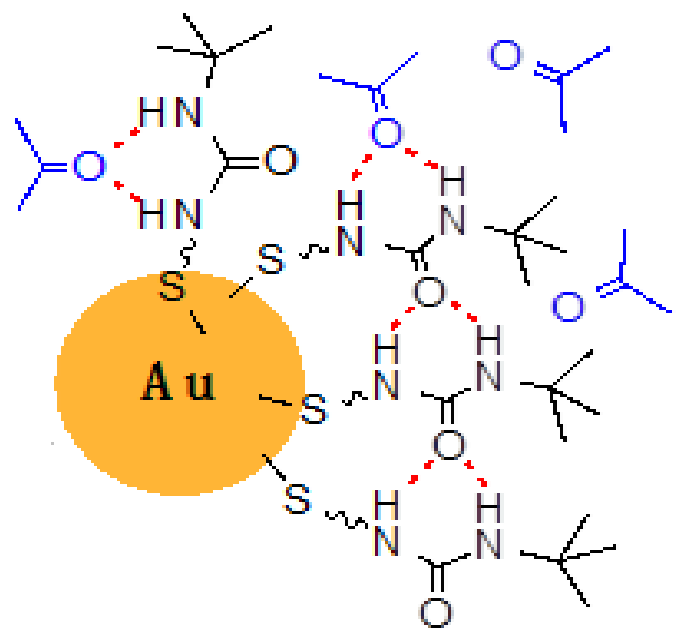

Figure 5.7 Hydrogen bonding interaction between AuNP with tert-butyl monoalkoxyurea thiol and acetone.

\subsection{Response Results for Alkylurea Thiol Sensor Sensing Acetone}

Figure 5.8 to 5.11 show the response data to acetone vapor in air for tert-butyl, cyclohexyl, phenyl and p-fluorophenyl alkylurea thiol sensors. 

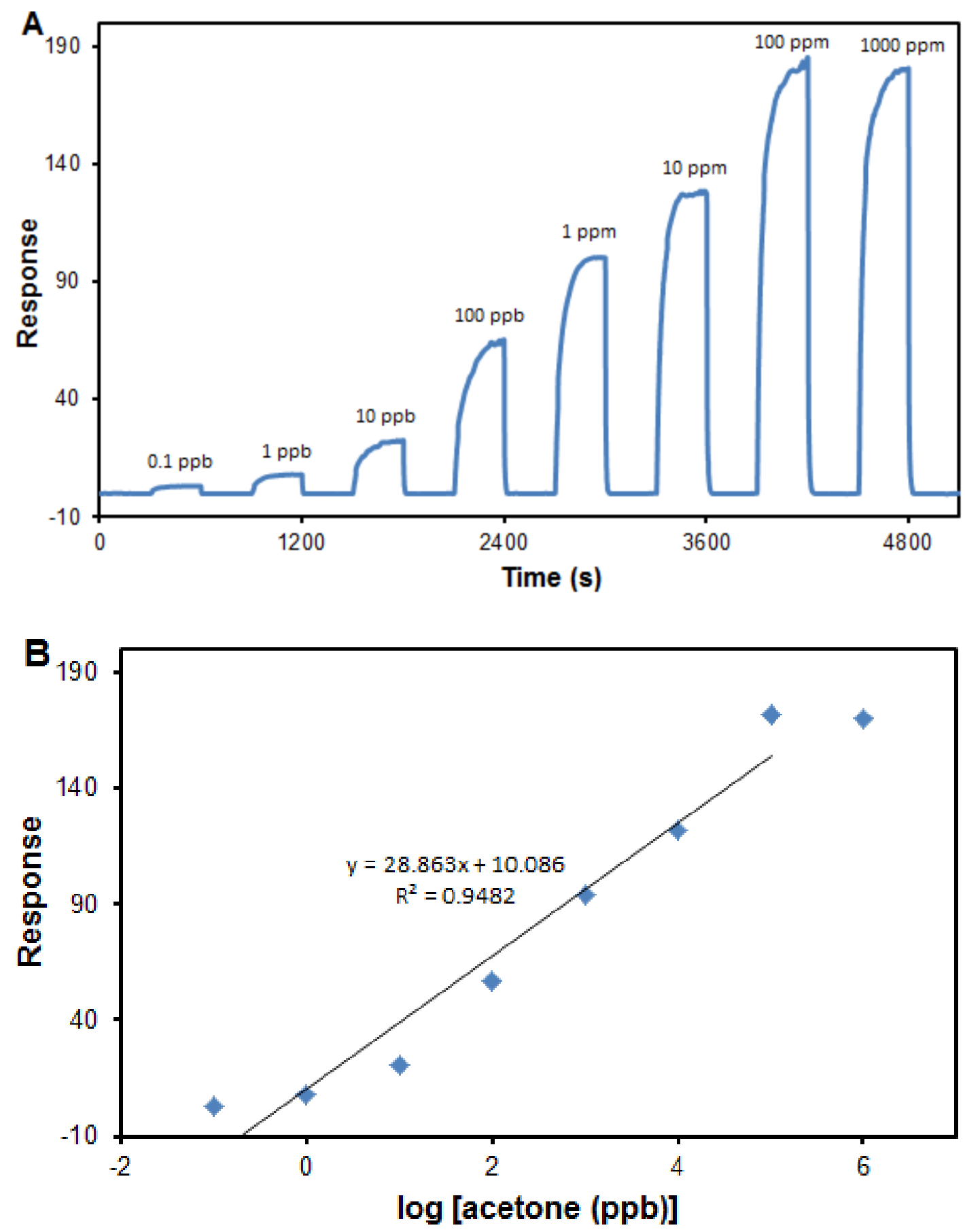

Figure 5.8 (A) tert-butyl alkylurea thiol sensor response profile in response to $0.1 \mathrm{ppb}$ to $1000 \mathrm{ppm}$ of acetone vapor. (B) Sensor response versus log[acetone (ppb)] in detection of different concentrations of acetone. 

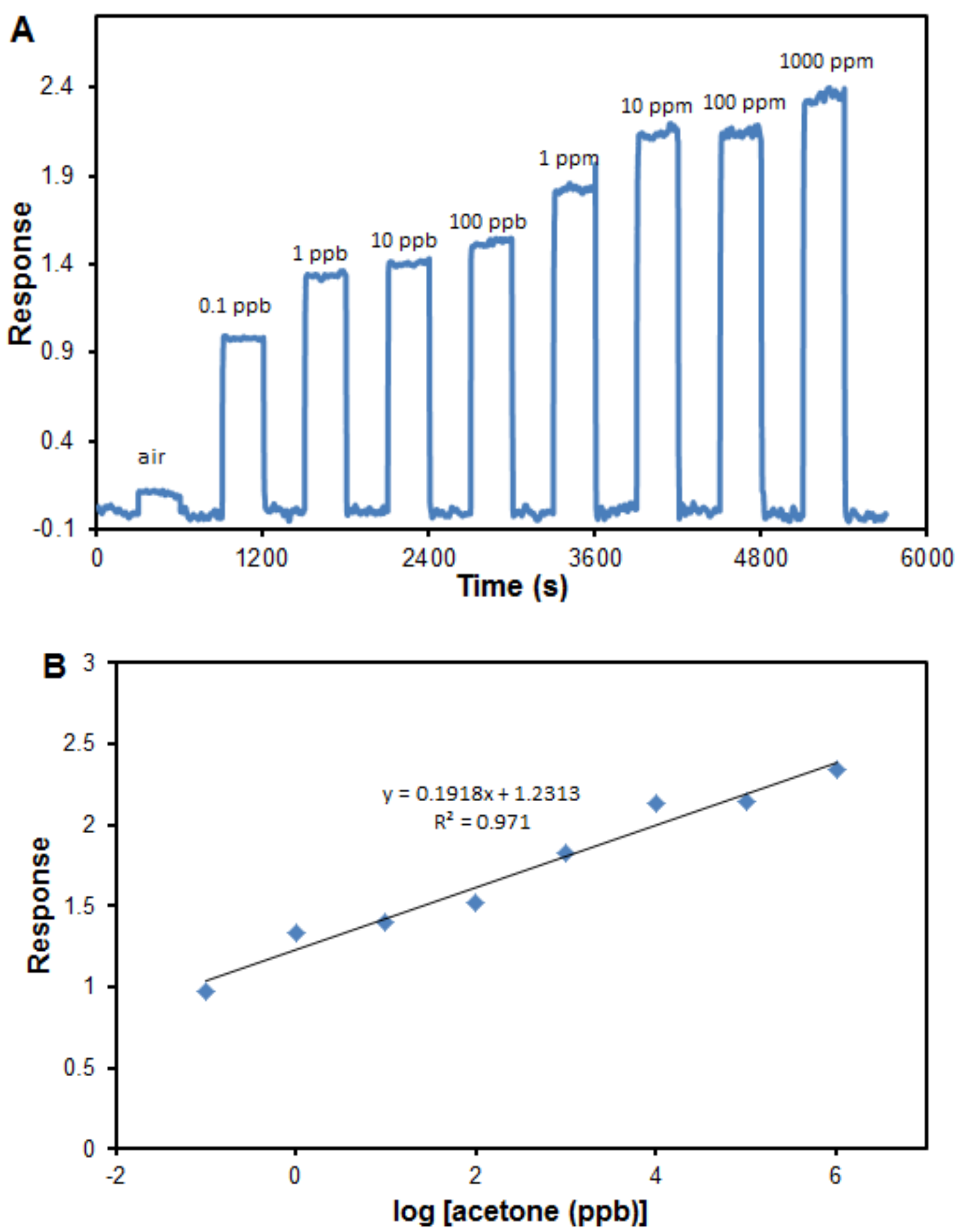

Figure 5.9 (A) Cyclohexyl alkylurea thiol sensor response profile in response to 0.1 ppb to $1000 \mathrm{ppm}$ of acetone vapor. (B) Sensor response versus log[acetone (ppb)] in detection of different concentrations of acetone 

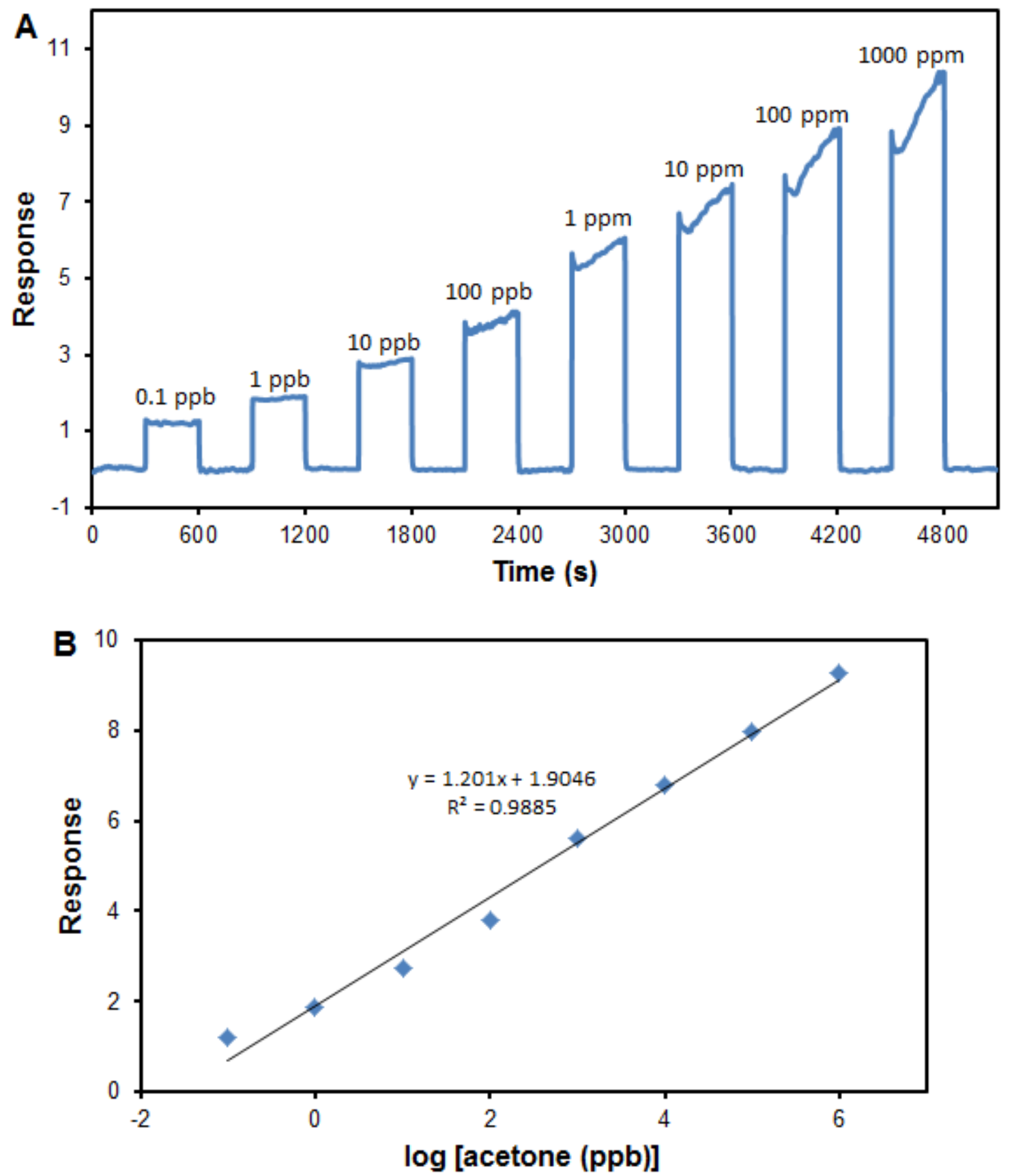

Figure 5.10 (A) Phenyl alkylurea thiol sensor response profile in response to $0.1 \mathrm{ppb}$ to $1000 \mathrm{ppm}$ of acetone vapor. (B) Sensor response versus $\log [$ acetone (ppb)] in detection of different concentrations of acetone 

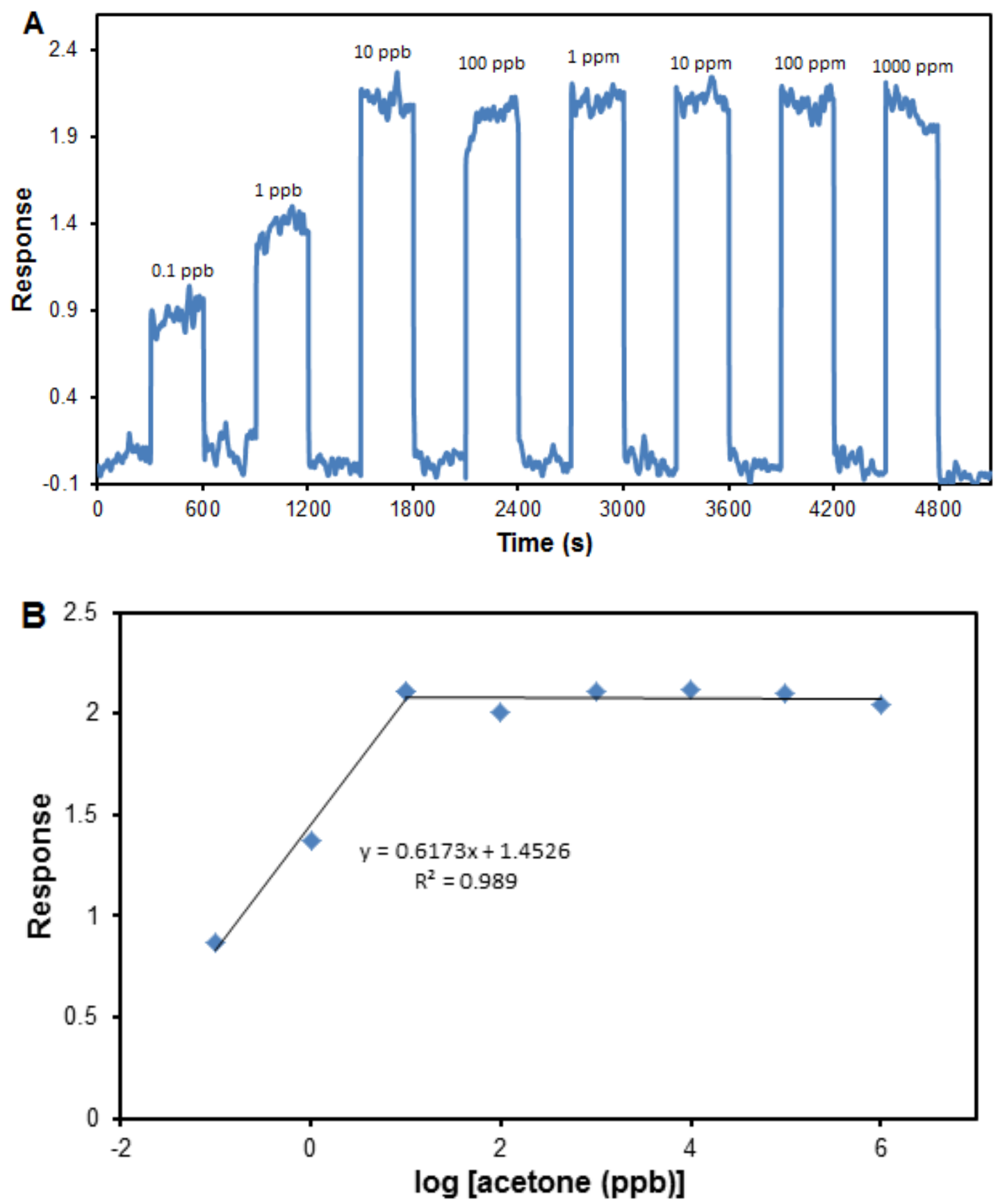

Figure 5.11 (A) p-fluorophenyl alkylurea thiol sensor response profile in response to $0.1 \mathrm{ppb}$ to $1000 \mathrm{ppm}$ of acetone vapor. (B) Sensor response versus log[acetone (ppb)] in detection of different concentrations of acetone 
Figure 5.8 to 5.11 show that $t$-butyl alkylurea thiol sensor is most sensitive to acetone in series 2 , which is the same as series 1 . The limit of detection of the sensors in series 2 is $0.1 \mathrm{ppb}$, which is different for cyclohexyl and phenyl alkylurea thiol from the first series. The response of p-fluorophenyl alkylurea thiol sensor is the lowest and saturates above $10 \mathrm{ppb}$.

In general, the response of alkylurea thiol sensor is larger than monoalkoxyurea thiol sensor. For example, the response of $1 \mathrm{ppm}$ acetone is 94 for $t$ butyl alkylurea thiol sensor and 13 for $t$-butyl monoalkoxyurea thiol sensor. The reason of this phenomenon can be explained by $\alpha$ - effect of an adjacent electronegative atom proposed by Edwards and Pearson [172,173]. The presence of an electronegative atom with one or more unshared pairs of electrons in an $\alpha$-position to nucleophilic atom was the controlling feature of overall nucleohilicity/acidity. Thus the unshared pair of electrons on O-atom stabilizes the acidity of urea $\mathrm{NH}$, which could ameliorate the H-bonding donating capability of urea NH. For example, in the case of monoalkoxyurea thiol, the $\alpha-\mathrm{O}$ atom makes the $-\mathrm{NH}-$ more acidic. This could form an efficient hydrogen bonding network, and the network between chains becomes too strong. Acetone is hard to get through and interact with AuNPs, thus shows lower response. As for alkylurea thiol sensor, the hydrogen bonding network is relatively weak, so acetone is easier to interact with the film, leading to higher response. 


\subsection{Response Results for bis-Alkoxyurea Thiol Sensor Sensing Acetone}

By comparing the 4 thiols functionalized AuNP sensors in series 1 with 4 thiols functionalized AuNP sensors in series 2, we tested tert-butyl and cyclohexyl bis-alkoxyurea thiol functionalized AuNP for series 3. Figure 5.12 to 5.13 show the response data to acetone vapor in air for tert-butyl and cyclohexyl bis-alkoxyurea thiol sensors. 

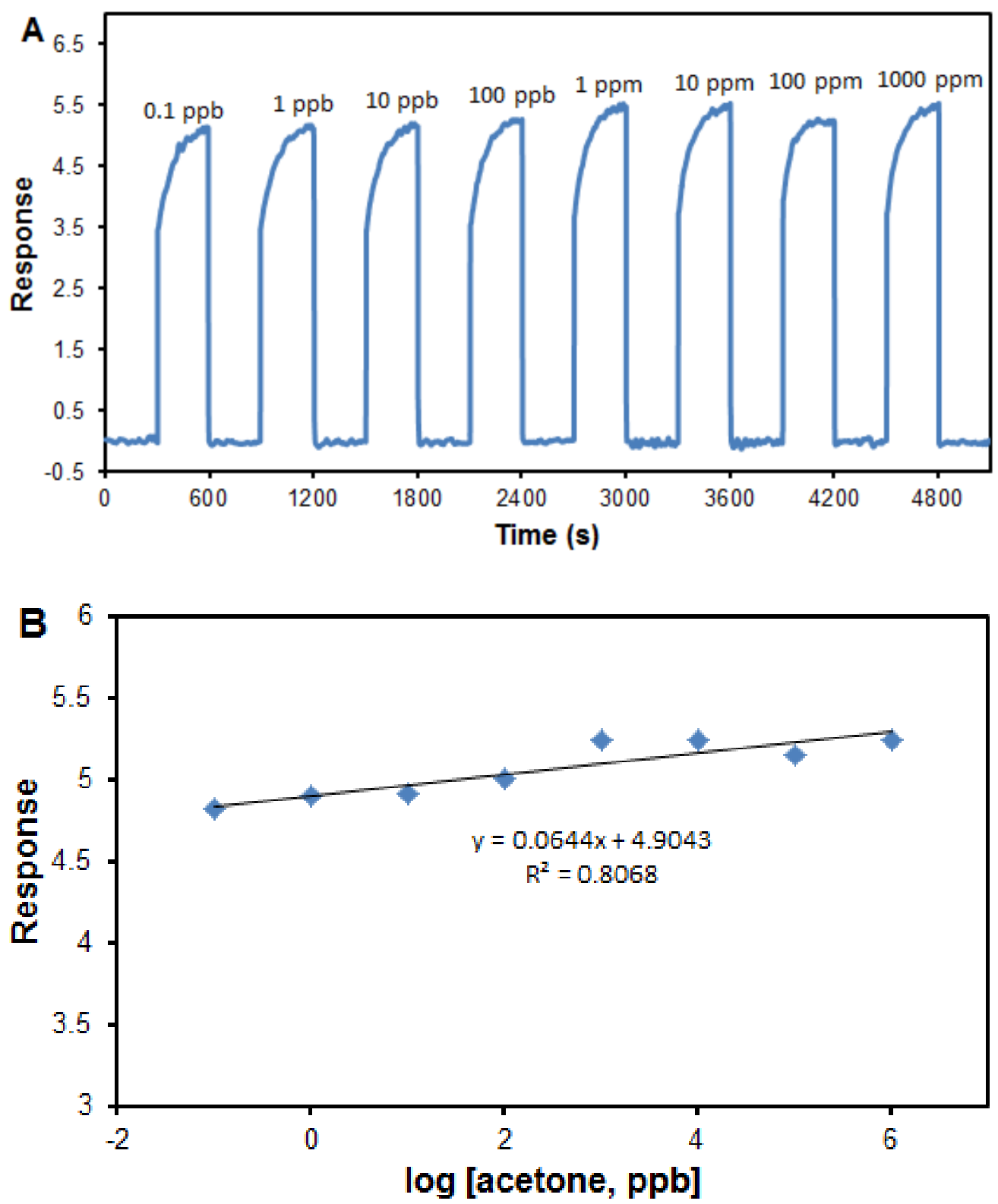

Figure 5.12 (A) tert-butyl bis-alkoxyurea thiol sensor response profile in response to $0.1 \mathrm{ppb}$ to $1000 \mathrm{ppm}$ of acetone vapor. (B) Sensor response versus log[acetone (ppb)] in detection of different concentrations of acetone 

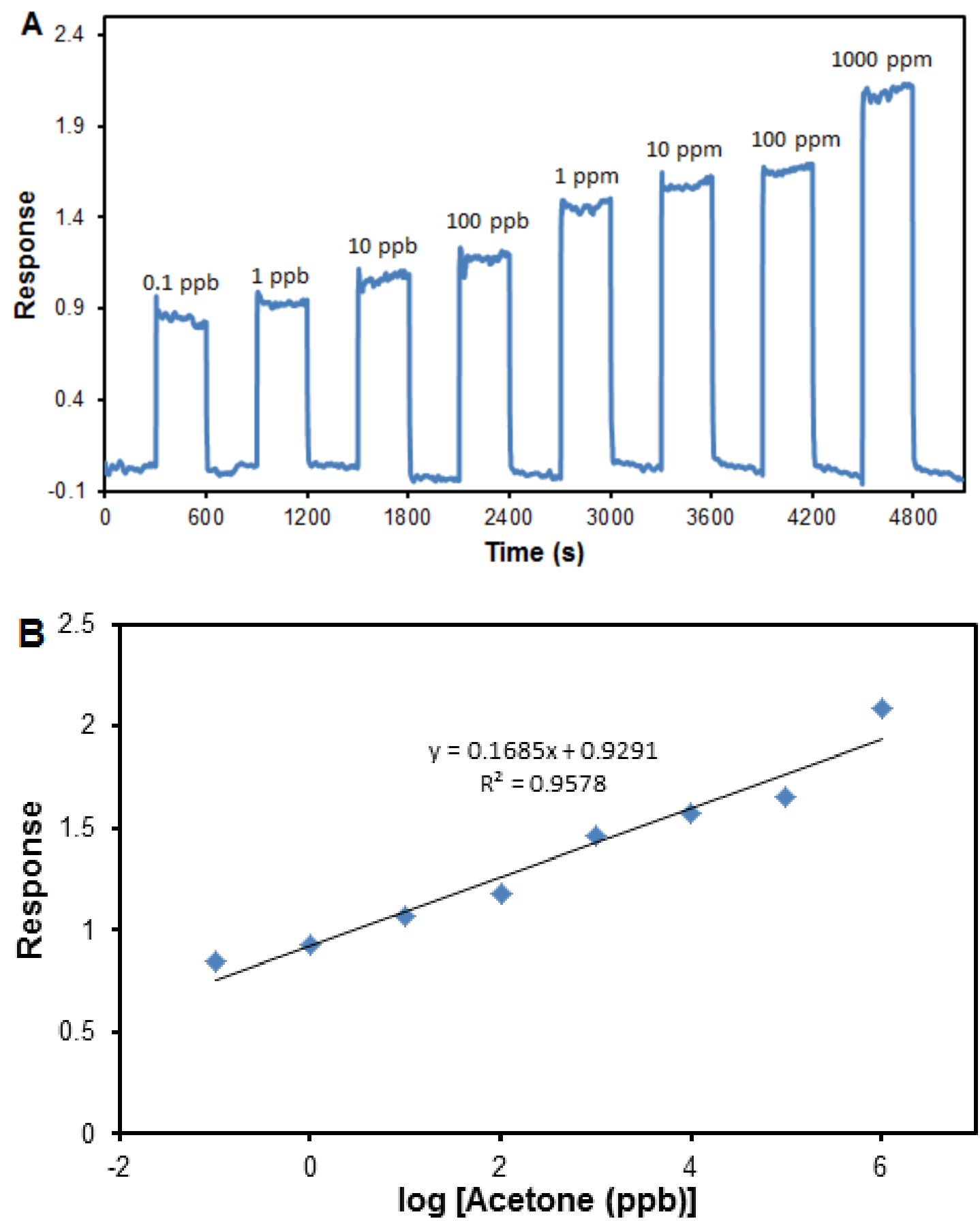

Figure 5.13 (A) Cyclohexyl bis-alkoxyurea thiol sensor response profile in response to $0.1 \mathrm{ppb}$ to $1000 \mathrm{ppm}$ of acetone vapor. (B) Sensor response versus log[acetone $(\mathrm{ppb})]$ in detection of different concentrations of acetone. 
Figure 5.12 and 5.13 show that the response for $t$-butyl bis-alkoxyurea thiol sensor is larger than that of cyclohexyl bis-alkoxyurea thiol sensor, which is consistent with the results for the other two series. However, the sensitivity (slope of the calibration curve) is low. Here, we use bar plot to compare the responses of three series of urea thiol sensors sensing $1 \mathrm{ppm}$ acetone (Figure 5.14). It is clearly shown that alkylurea thiol sensors exhibit the highest response for any functional groups. Both electronic and steric effects need to be considered here. In bis-alkoxyurea thiol sensors, two $\alpha-\mathrm{O}$ atoms make $-\mathrm{NH}-$ even more acidic than monoalkoxyurea thiol sensors [174-177], and the networks between chains are stronger. Acetone is hard to access the network, so bis-alkoxyurea thiol sensors show lowest response among three series. For different control groups, tert-butyl group is bulky and the network is disrupted, which makes hydrogen bonding network between chains weak, and allows acetone access. 


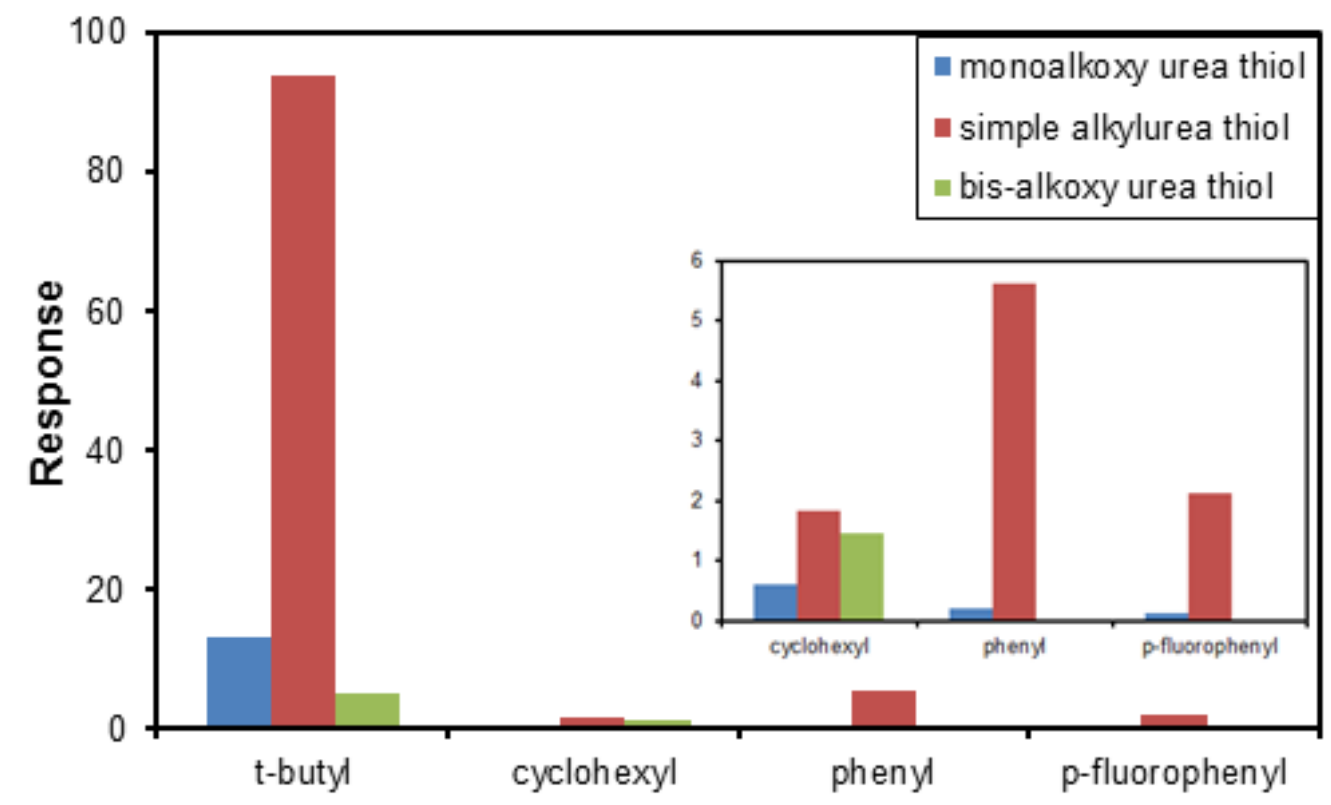

Figure 5.14 The response patterns for three series urea thiol sensors sensing $1 \mathrm{ppm}$ acetone. The inserted graph is a magnified view of the response below 6 .

\section{Selectivity of tert-butyl Alkylurea Thiol Sensor for Acetone}

Given the high sensitivity of tert-butyl alkylurea thiol sensor toward acetone, we set out to test the acetone-sensing selectivity of the sensor by measuring sensor responses to ethanol and benzene (Figure 5.15). As the slope of the linear regression curve is a direct measure of sensitivity, the relatively flat slopes obtained for ethanol (0.39) and benzene (2.52) imply that the sensor discriminates in its interaction with VOCs. The stronger sensitivity for tert-butyl alkylurea thiol toward acetone (slope $=$ 33.2) than tert-butyl monoalkoxyurea thiol (slope $=4.6$ ) and tert-butyl bis-alkoxyurea thiol sensor (slope $=0.064$ ) indicates that the hydrogen bonding interaction is affected by electronic factor. 


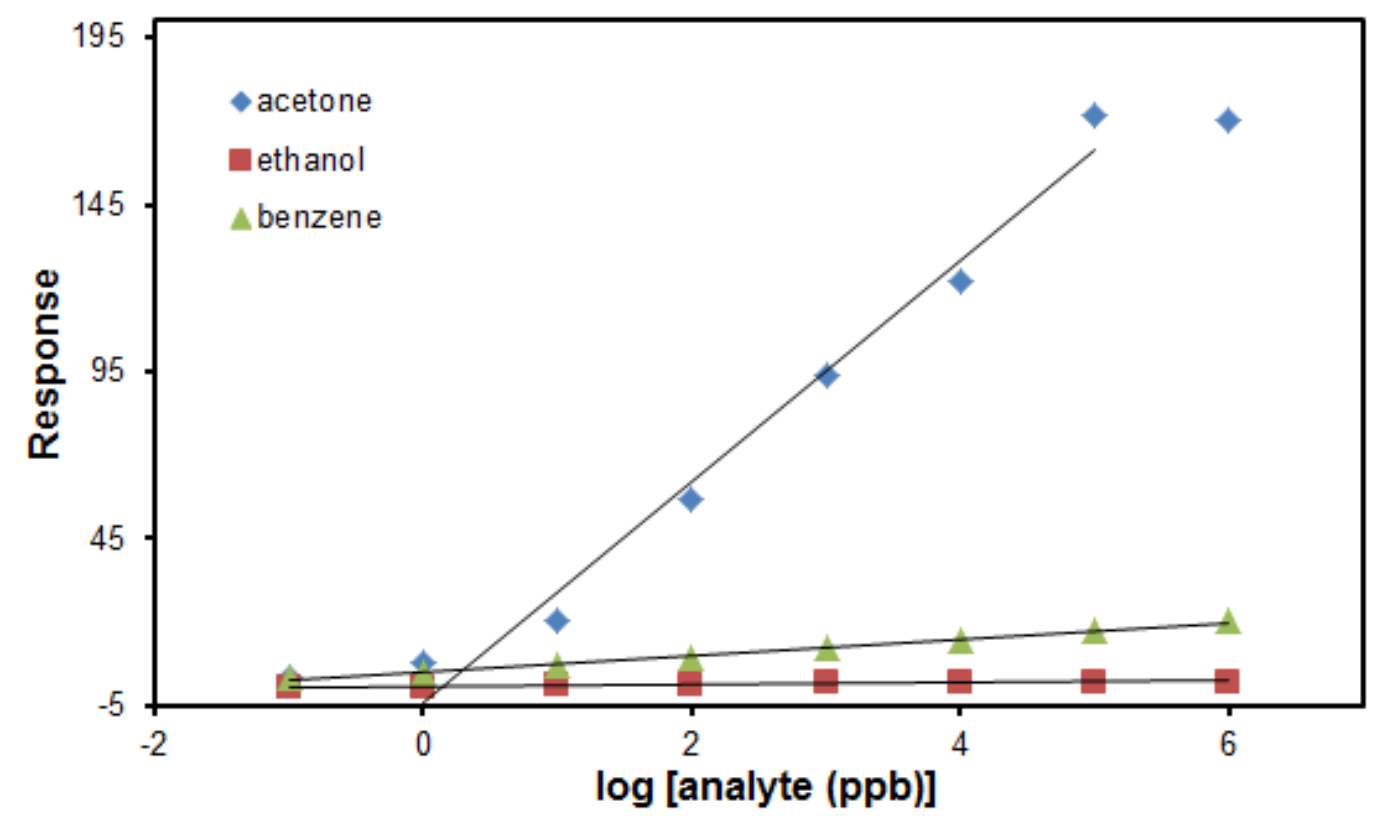

Figure 5.15 tert-butyl alkylurea thiol sensor response versus $\log [$ analyte (ppb)] in detection of acetone, ethanol and benzene

\section{Conclusions}

In conclusion, we have prepared panels of monoalkoxyurea thiols, alkylurea thiols and bis-alkoxyurea thiols that differ principally in the control group $\mathrm{R}$ to develop optimal acetone-binding substrates for attachment of gold nanoparticles at ambient temperature. From our results, we found out that in general alkylurea thiols functionalized gold nanoparticle sensors show the highest sensitivity, among which the control group tert-butyl exhibits largest sensitivity and selectivity toward acetone with the detection limit of $0.1 \mathrm{ppb}$. The concept of designed thiols for selective interaction with target analytes based on functional group interactions, such as urea- 
carbonyl hydrogen bonding helps improve the chemiresistor sensitivity and selectivity. Promising would be also its application under real-word conditions. 


\section{CHAPTER VI}

\section{SUMMARY AND FUTURE WORK}

This dissertation has investigated gas sensors based on gold nanoparticles functionalized with thiol motifs for detecting volatile organic compounds in both air and exhaled breath.

\section{Summary}

Nanoparticle-structured sensing arrays provide new opportunities for enhancing the design of sensor materials in terms of sensitivity and selectivity. The device is inexpensive, portable and amenable to use, making them potentially valuable in medical diagnosis. In this work, we have demonstrated the capability of nanostructured gas sensors derived from gold nanoparticles functionalized with thiol assemblies for the detection of VOCs. Gas sensing measurements reveal that the sensors show the lowest limit of detection to acetone among the reported chemiresistors to date. We have designed new thiol motifs to functionalize gold nanoparticles for sensing VOCs, which dramatically increases both the sensitivity and 
selectivity of the sensors for sensing trace carbonyl compounds in air and exhaled breath.

AuNPs were synthesized at room temperature using a two-phase reduction approach. Interdigitated electrodes were designed using CAD program and fabricated using lift-off microfabrication technique. The results of sensor measurement show that the nanostructured sensors display linear responses to the target analyte concentrations. We used different alkyl chain length of the thiol monolayer surrounding the AuNPs to control the electronic properties of the sensing film. We found that films containing larger number of alkyl chain carbons showed greater responses and sensitivities to VOCs in a range from $0.1 \mathrm{ppb}$ to $1000 \mathrm{ppm}$. Because of the poor sensitivity and selectivity of the commercial thiols functionalized AuNP sensors, we have developed new thiol ligands fitted with a $\omega$-urea group, which provide the unique and parallel-oriented hydrogen bonding networks formed by urea moieties and the carbonyl compounds. To the best of our knowledge, it is the first time that the thiol with urea-functional motif is applied to coat on AuNP for sensing purpose.

The sensor properties were measured in both air and exhaled breath samples. Humidity effect on the sensors was discussed. Urea thiol sensor shows the largest response change with $\mathrm{RH}$ increasing from 0 to $90 \%$, while 1-dodecanethiol sensor shows the least response change. In this case, the increase in water vapor concentration during breath sampling could lead to false acetone detection. However, at the same background of other compounds, our sensors were able to recognize different concentrations of acetone, which is promising for diagnosing of diabetes. 
Thus, it's better to use adsorbents to remove water vapor before testing high humidity samples.

Based on the good sensitivity and selectivity of monoalkoxyurea thiol sensor, we have developed another two series of urea motifs for detecting of acetone, which are alkylurea thiols and bis-alkoxyurea thiols with different control groups. The synthesis procedures and the sensor measurements of all the three series of thiols have been presented. The slope of the linear regression curve is a direct measure of sensitivity. The results show that the sensitivity is the highest with functional group of tert-butyl in each series of thiols because of its steric effect. Among the three series, sensors functionalized with alkylurea thiols have the best sensitivity, which indicate that the response is affected by its electronic effect. The response of ethanol and benzene were also measured to determine the selectivity. The relative flat slopes obtained for ethanol (0.39) and benzene (2.52) imply the alkyurea thiol sensor discriminates these VOCs through its interaction with them. The considerably stronger sensitivity for tert-butyl alkylurea thiol sensor to acetone (slope=33.2) suggests the hydrogen bonding design concept is operative and imparts both sensitivity and selectivity. The results have provided us an important noninvasive tool for diabetes screening and diagnosis in the future.

\section{Future Directions}

There are several areas or directions in the near future, which we could further enhance the work presented in this dissertation. 
1. Gold nanoparticle shape may also play an important role in the gas sensing properties. In this work, we have reported the results for spherical AuNPs with diameter of $\sim 3 \mathrm{~nm}$. The shape of the nanoparticles can be changed to nanorods, nanocubes, nanostars or nanotriangles. The size of gold nanoparticles can also be varied.

2. As the respective sensors are developed, extrapolation to develop motifs for detecting other volatile organic compounds can be pursued. For example, arenebinding substrates can be synthesized for detecting aromatic hydrocarbon (benzene) analytes. The use of $\pi-\pi$ interactions ( $\pi$ stacking) is another important interaction used for molecular recognition. The strong $\pi$-stacking interactions of the tetraphenylethylene unit of the arene binding motif can be exploited. In this manner, we can enhance the sensitivity and selectivity for the gold nanosensor array to benzene as well as other aromatic hydrocarbons.

3. As discussed before, human breath contains more than $90 \%$ of humidity and our sensor shows large response under high humidity. Adsorbents need to be used to remove water before the measurement. Also, in our study, we used simulated diabetes breath sample for the measurements. In future, real diabetic patient's breath samples can be applied for the sensor to differentiate it from healthy controls. Many VOCs were found as the biomarkers of a certain disease. If we can synthesis different thiols which show high sensitivity and selectivity to the biomarkers, it's promising for us to diagnose the disease at early stage.

4. The long term stability of the sensors needs to be achieved for deployment in real world applications. Our sensors were stable to acetone for only 15 days 
because the oxidation of the surface thiolate occurs when exposed to air, light and moisture under ambient conditions. Trithiol ligands may need to be synthesized to functionalize AuNP in order to overcome this limitation. The deterioration rate of trithiolate ligand should be significantly slower since the chance of three thiolate groups being completely oxidized and desorbed is significantly lower. Therefore, the aggregation of nanoparticles is highly restrained. Also, the higher binding energy of trithiol-AuNPs might be another factor that contributes to the particular stability. 


\section{REFERENCES}

1. Cao, W.Q. and Duan, Y.X., Current status of methods and techniques for breath analysis. Critical Reviews in Analytical Chemistry, 2007. 37(1): p. 313.

2. Amann, A., Spanel, P., and Smith, D., Breath Analysis: The Approach Towards Clinical Applications. Mini-Reviews in Medicinal Chemistry, 2007. 7(2): p. $115-129$.

3. Braun, P.X., Gmachl, C.F., and Dweik, R.A., Bridging the Collaborative Gap: Realizing the Clinical Potential of Breath Analysis for Disease Diagnosis and Monitoring-Tutorial. Ieee Sensors Journal, 2012. 12(11): p. 3258-3270.

4. Dweik, R.A. and Amann, A., Exhaled breath analysis: the new frontier in medical testing. Journal of Breath Research, 2008. 2(3).

5. Libardoni, M., Stevens, P.T., Waite, J.H., and Sacks, R., Analysis of human breath samples with a multi-bed sorption trap and comprehensive twodimensional gas chromatography $(G C \times G C)$. Journal of Chromatography BAnalytical Technologies in the Biomedical and Life Sciences, 2006. 842(1): p. $13-21$. 
6. Di Francesco, F., Fuoco, R., Trivella, M.G., and Ceccarini, A., Breath analysis: trends in techniques and clinical applications. Microchemical Journal, 2005. 79(1-2): p. 405-410.

7. Pauling, L., Robinson, A.B., Teranish.R, and Cary, P., Quantitative Analysis of Urine Vapor and Breath by Gas-Liquid Partition Chromatography. Proceedings of the National Academy of Sciences of the United States of America, 1971. 68(10): p. 2374-\&.

8. Manolis, A., The Diagnostic Potential of Breath Analysis. Clinical Chemistry, 1983. 29(1): p. 5-15.

9. Hamid, Q., Springall, D.R., Riverosmoreno, V., Chanez, P., Howarth, P., Redington, A., Bousquet, J., Godard, P., Holgate, S., and Polak, J.M., Induction of Nitric-Oxide Synthase in Asthma. Lancet, 1993. 342(8886-7): p. $1510-1513$.

10. Kharitonov, S.A., Yates, D., Robbins, R.A., Logansinclair, R., Shinebourne, E.A., and Barnes, P.J., Increased Nitric-Oxide in Exhaled Air of AsthmaticPatients. Lancet, 1994. 343(8890): p. 133-135.

11. Massaro, A.F., Gaston, B., Kita, D., Fanta, C., Stamler, J.S., and Drazen, J.M., Expired Nitric-Oxide Levels during Treatment of Acute Asthma. American Journal of Respiratory and Critical Care Medicine, 1995. 152(2): p. 800-803.

12. Machado, R.F., Laskowski, D., Deffenderfer, O., Burch, T., Zheng, S., Mazzone, P.J., Mekhail, T., Jennings, C., Stoller, J.K., Pyle, J., Duncan, J., Dweik, R.A., and Erzurum, S.C., Detection of lung cancer by sensor array 
analyses of exhaled breath. American Journal of Respiratory and Critical Care Medicine, 2005. 171(11): p. 1286-1291.

13. Buszewski, B., Kesy, M., Ligor, T., and Amann, A., Human exhaled air analytics: Biomarkers of diseases. Biomedical Chromatography, 2007. 21(6): p. 553-566.

14. Pandey, S.K. and Kim, K.H., Human body-odor components and their determination. Trac-Trends in Analytical Chemistry, 2011. 30(5): p. 784-796.

15. Dolch, M.E., Hornuss, C., Klocke, C., Praun, S., Villinger, J., Denzer, W., Schelling, G., and Schubert, S., Volatile organic compound analysis by ion molecule reaction mass spectrometry for Gram-positive bacteria differentiation. European Journal of Clinical Microbiology \& Infectious Diseases, 2012. 31(11): p. 3007-3013.

16. Rooth, G., Ostenson, S., Acetone in alveolar air, and the control of diabetes. The Lancet, 1966. 288: p. 1102-1105.

17. Tassopoulos, C.N., Barnett, D., Fraser, T.R., Breath-acetone and blood-sugar measurements in diabetes. The lancet, 1969. 293: p. 1282-1286.

18. Deng, C.H., Zhang, J., Yu, X.F., Zhang, W., and Zhang, X.M., Determination of acetone in human breath by gas chromatography-mass spectrometry and solid-phase microextraction with on-fiber derivatization. Journal of Chromatography B-Analytical Technologies in the Biomedical and Life Sciences, 2004. 810(2): p. 269-275.

19. Peng, G., Tisch, U., Adams, O., Hakim, M., Shehada, N., Broza, Y.Y., Billan, S., Abdah-Bortnyak, R., Kuten, A., and Haick, H., Diagnosing lung cancer in 
exhaled breath using gold nanoparticles. Nature Nanotechnology, 2009. 4(10): p. 669-673.

20. Ruzsanyi, V., Baumbach, J.I., Sielemann, S., Litterst, P., Westhoff, M., and Freitag, L., Detection of human metabolites using multi-capillary columns coupled to ion mobility spectrometers. Journal of Chromatography A, 2005. 1084(1-2): p. 145-151.

21. Periago, J.F., Prado, C., Ibarra, I., and Tortosa, J., Application of ThermalDesorption to the Biological Monitoring of Organic-Compounds in Exhaled Breath. Journal of Chromatography A, 1993. 657(1): p. 147-153.

22. Prado, C., Marin, P., and Periago, J.F., Application of solid-phase microextraction and gas chromatography-mass spectrometry to the determination of volatile organic compounds in end-exhaled breath samples. Journal of Chromatography A, 2003. 1011(1-2): p. 125-134.

23. Grote, C. and Pawliszyn, J., Solid-phase microextraction for the analysis of human breath. Analytical Chemistry, 1997. 69(4): p. 587-596.

24. De Hoffmann, E., Charette, J. and Stroobant, V., Mass Spectrometry. Principles and Applications. Wiley: New York, 1996.

25. Paul, W.a.S., H. , US. Patent 2, 1960.

26. Boots, A.W., van Berkel, J.J.B.N., Dallinga, J.W., Smolinska, A., Wouters, E.F., and van Schooten, F.J., The versatile use of exhaled volatile organic compounds in human health and disease. Journal of Breath Research, 2012. 6(2). 
27. Van Berkel, J.J.B.N., Dalling, J.W., Moeller, G.M., Godschalk, R.W.L., Moonen, E., Wouters, E.F.M., and Van Schooten, F.J., Development of accurate classification method based on the analysis of volatile organic compounds from human exhaled air. Journal of Chromatography B-Analytical Technologies in the Biomedical and Life Sciences, 2008. 861(1): p. 101-107.

28. Mieth, M., Schubert, J.K., Groger, T., Sabel, B., Kischkel, S., Fuchs, P., Hein, D., Zimmermann, R., and Miekisch, W., Automated Needle Trap Heart-Cut GC/MS and Needle Trap Comprehensive Two-Dimensional GC/TOF-MS for Breath Gas Analysis in the Clinical Environment. Analytical Chemistry, 2010. 82(6): p. 2541-2551.

29. Ibrahim, B., Basanta, M., Cadden, P., Singh, D., Douce, D., Woodcock, A., and Fowler, S.J., Non-invasive phenotyping using exhaled volatile organic compounds in asthma. Thorax, 2011. 66(9): p. 804-809.

30. Trefz, P., Rosner, L., Hein, D., Schubert, J.K., and Miekisch, W., Evaluation of needle trap micro-extraction and automatic alveolar sampling for point-ofcare breath analysis (vol 405, pg 3105, 2013). Analytical and Bioanalytical Chemistry, 2013. 405(16): p. 5617-5617.

31. Amann, A., Poupart, G., Telser, S., Ledochowski, M., Schmid, A., and Mechtcheriakov, S., Applications of breath gas analysis in medicine. International Journal of Mass Spectrometry, 2004. 239(2-3): p. 227-233.

32. Zhan, X.F., Duan, J.N., and Duan, Y.X., Recent Developments of ProtonTransfer Reaction Mass Spectrometry (Ptr-Ms) and Its Applications in Medical Research. Mass Spectrometry Reviews, 2013. 32(2): p. 143-165. 
33. Smith, D. and Spanel, P., Selected ion flow tube mass spectrometry (SIFT-MS) for on-line trace gas analysis. Mass Spectrometry Reviews, 2005. 24(5): p. $661-700$.

34. Kumar, S., Huang, J.Z., Abbassi-Ghadi, N., Spanel, P., Smith, D., and Hanna, G.B., Selected Ion Flow Tube Mass Spectrometry Analysis of Exhaled Breath for Volatile Organic Compound Profiling of Esophago-Gastric Cancer. Analytical Chemistry, 2013. 85(12): p. 6121-6128.

35. Spanel, P. and Smith, D., Selected ion flow tube mass spectrometry for on-line trace gas analysis in biology and medicine. European Journal of Mass Spectrometry, 2007. 13(1): p. 77-82.

36. Taucher, J., Hansel, A., Jordan, A., Fall, R., Futrell, J.H., and Lindinger, W., Detection of isoprene in expired air from human subjects using protontransfer-reaction mass spectrometry. Rapid Communications in Mass Spectrometry, 1997. 11(11): p. 1230-1234.

37. Hansel, A., Jordan, A., Holzinger, R., Prazeller, P., Vogel, W., and Lindinger, W., Proton-Transfer Reaction Mass-Spectrometry - Online Trace GasAnalysis at the Ppb Level. International Journal of Mass Spectrometry, 1995. 149: p. 609-619.

38. Schwoebel, H., Schubert, R., Sklorz, M., Kischkel, S., Zimmermann, R., Schubert, J.K., and Miekisch, W., Phase-resolved real-time breath analysis during exercise by means of smart processing of PTR-MS data. Analytical and Bioanalytical Chemistry, 2011. 401(7): p. 2079-2091. 
39. Wehinger, A., Schmid, A., Mechtcheriakov, S., Ledochowski, M., Grabmer, C., Gastl, G.A., and Amann, A., Lung cancer detection by proton transfer reaction mass-spectrometric analysis of human breath gas. International Journal of Mass Spectrometry, 2007. 265(1): p. 49-59.

40. Blake, R.S., Whyte, C., Hughes, C.O., Ellis, A.M., and Monks, P.S., Demonstration of proton-transfer reaction time-of-flight mass spectrometry for real-time analysis of trace volatile organic compounds. Analytical Chemistry, 2004. 76(13): p. 3841-3845.

41. Hewitt, C.N., Hayward, S., and Tani, A., The application of proton transfer reaction-mass spectrometry (PTR-MS) to the monitoring and analysis of volatile organic compounds in the atmosphere. Journal of Environmental Monitoring, 2003. 5(1): p. 1-7.

42. Watson, J.T., Sparkman, O.D., Introduction to Mass Spectrometry: Instrumentation, Applications and Strategies for Data Interpretation. 2007.

43. Westhoff, M., Litterst, P., Freitag, L., Urfer, W., Bader, S., and Baumbach, J.I., Ion mobility spectrometry for the detection of volatile organic compounds in exhaled breath of patients with lung cancer: results of a pilot study. Thorax, 2009. 64(9): p. 744-748.

44. Baumbach, J.I., Ion mobility spectrometry coupled with multi-capillary columns for metabolic profiling of human breath. Journal of Breath Research, 2009. 3(3). 
45. Brodrick, E., Davies, A., Neill, P., Hanna, L., and Williams, E.M., Breath analysis: translation into clinical practice. Journal of Breath Research, 2015. 9(2).

46. Cui, Y., Wei, Q.Q., Park, H.K., and Lieber, C.M., Nanowire nanosensors for highly sensitive and selective detection of biological and chemical species. Science, 2001. 293(5533): p. 1289-1292.

47. Wan, Q., Li, Q.H., Chen, Y.J., Wang, T.H., He, X.L., Li, J.P., and Lin, C.L., Fabrication and ethanol sensing characteristics of $\mathrm{ZnO}$ nanowire gas sensors. Applied Physics Letters, 2004. 84(18): p. 3654-3656.

48. Kolmakov, A., Zhang, Y.X., Cheng, G.S., and Moskovits, M., Detection of $\mathrm{CO}$ and O-2 using tin oxide nanowire sensors. Advanced Materials, 2003. 15(12): p. 997-+.

49. Righettoni, M., Tricoli, A., and Pratsinis, S.E., Si:WO3 Sensors for Highly Selective Detection of Acetone for Easy Diagnosis of Diabetes by Breath Analysis. Analytical Chemistry, 2010. 82(9): p. 3581-3587.

50. Qin, L.P., Xu, J.Q., Dong, X.W., Pan, Q.Y., Cheng, Z.X., Xiang, Q., and Li, F., The template-free synthesis of square-shaped $\mathrm{SnO}(2)$ nanowires: the temperature effect and acetone gas sensors. Nanotechnology, 2008. 19(18).

51. Yan, S.H., Ma, S.Y., Li, W.Q., Xu, X.L., Cheng, L., Song, H.S., and Liang, X.Y., Synthesis of SnO2-ZnO heterostructured nanofibers for enhanced ethanol gas-sensing performance. Sensors and Actuators B-Chemical, 2015. 221: p. 88-95. 
52. Bhowmik, B., Manjuladevi, V., Gupta, R.K., and Bhattacharyya, P., Highly Selective Low-Temperature Acetone Sensor Based on Hierarchical 3-D TiO2 Nanoflowers. Ieee Sensors Journal, 2016. 16(10): p. 3488-3495.

53. Tomer, V.K. and Duhan, S., Ordered mesoporous Ag-doped TiO2/SnO2 nanocomposite based highly sensitive and selective VOC sensors. Journal of Materials Chemistry A, 2016. 4(3): p. 1033-1043.

54. Al-Hardan, N.H., Abdullah, M.J., and Aziz, A.A., Performance of Cr-doped $\mathrm{ZnO} \mathrm{for} \mathrm{acetone} \mathrm{sensing.} \mathrm{Applied} \mathrm{Surface} \mathrm{Science,} \mathrm{2013.} \mathrm{270:} \mathrm{p.} \mathrm{480-485.}$

55. Rella, R., Spadavecchia, J., Manera, M.G., Capone, S., Taurino, A., Martino, M., Caricato, A.P., and Tunno, T., Acetone and ethanol solid-state gas sensors based on TiO2 nanoparticles thin film deposited by matrix assisted pulsed laser evaporation. Sensors and Actuators B-Chemical, 2007. 127(2): p. 426431.

56. Geng, L., Zhao, Y.Q., Huang, X.L., Wang, S.R., Zhang, S.M., and Wu, S.H., Characterization and gas sensitivity study of polyaniline/SnO2 hybrid material prepared by hydrothermal route. Sensors and Actuators B-Chemical, 2007. 120(2): p. 568-572.

57. Park, J.S., Shen, X.P., and Wang, G.X., Solvothermal synthesis and gassensing performance of $\operatorname{Co}(3) O(4)$ hollow nanospheres. Sensors and Actuators B-Chemical, 2009. 136(2): p. 494-498.

58. Teleki, A., Pratsinis, S.E., Kalyanasundaram, K., and Gouma, P.I., Sensing of organic vapors by flame-made TiO2 nanoparticles. Sensors and Actuators BChemical, 2006. 119(2): p. 683-690. 
59. Ge, C.Q., Xie, C.S., and Cai, S.Z., Preparation and gas-sensing properties of Ce-doped ZnO thin-film sensors by dip-coating. Materials Science and Engineering B-Solid State Materials for Advanced Technology, 2007. 137(13): p. 53-58.

60. Snow, E.S., Perkins, F.K., and Robinson, J.A., Chemical vapor detection using single-walled carbon nanotubes. Chemical Society Reviews, 2006. 35(9): p. 790-798.

61. Pengfei, Q.F., Vermesh, O., Grecu, M., Javey, A., Wang, O., Dai, H.J., Peng, S., and Cho, K.J., Toward large arrays of multiplex functionalized carbon nanotube sensors for highly sensitive and selective molecular detection. Nano Letters, 2003. 3(3): p. 347-351.

62. Sysoev, V.V., Button, B.K., Wepsiec, K., Dmitriev, S., and Kolmakov, A., Toward the nanoscopic "electronic nose": Hydrogen vs carbon monoxide discrimination with an array of individual metal oxide nano- and mesowire sensors. Nano Letters, 2006. 6(8): p. 1584-1588.

63. Pinto, N.J., Ramos, I., Rojas, R., Wang, P.C., and Johnson, A.T., Electric response of isolated electrospun polyaniline nanofibers to vapors of aliphatic alcohols. Sensors and Actuators B-Chemical, 2008. 129(2): p. 621-627.

64. Li, C.L., Chartuprayoon, N., Bosze, W., Low, K., Lee, K.H., Nam, J., and Myung, N.V., Electrospun Polyaniline/Poly(ethylene oxide) Composite Nanofibers Based Gas Sensor. Electroanalysis, 2014. 26(4): p. 711-722.

65. Vallejos, S., Gracia, I., Bravo, J., Figueras, E., Hubalek, J., and Cane, C., Detection of volatile organic compounds using flexible gas sensing devices 
based on tungsten oxide nanostructures functionalized with $A u$ and Pt nanoparticles. Talanta, 2015. 139: p. 27-34.

66. Han, L., Shi, X.J., Wu, W., Kirk, F.L., Luo, J., Wang, L.Y., Mott, D., Cousineau, L., Lim, S.I.I., Lu, S., and Zhong, C.J., Nanoparticle-structured sensing array materials and pattern recognition for VOC detection. Sensors and Actuators B-Chemical, 2005. 106(1): p. 431-441.

67. Ahn, H., Chandekar, A., Kang, B., Sung, C., and Whitten, J.E., Electrical conductivity and vapor-sensing properties of omega-(3-thienyl)alkanethiolprotected gold nanoparticle films. Chemistry of Materials, 2004. 16(17): p. 3274-3278.

68. Ibanez, F.J. and Zamborini, F.P., Chemiresistive Sensing with Chemically Modified Metal and Alloy Nanoparticles. Small, 2012. 8(2): p. 174-202.

69. Fleischer, M., Simon, E., Rumpel, E., Ulmer, H., Harbeck, M., Wandel, M., Fietzek, C., Weimar, U., and Meixner, H., Detection of volatile compounds correlated to human diseases through breath analysis with chemical sensors. Sensors and Actuators B-Chemical, 2002. 83(1-3): p. 245-249.

70. Di Natale, C., Macagnano, A., Martinelli, E., Paolesse, R., D'Arcangelo, G., Roscioni, C., Finazzi-Agro, A., and D'Amico, A., Lung cancer identification by the analysis of breath by means of an array of non-selective gas sensors. Biosensors \& Bioelectronics, 2003. 18(10): p. 1209-1218.

71. Ryabtsev, S.V., Shaposhnick, A.V., Lukin, A.N., and Domashevskaya, E.P., Application of semiconductor gas sensors for medical diagnostics. Sensors and Actuators B-Chemical, 1999. 59(1): p. 26-29. 
72. Wang, L., Teleki, A., Pratsinis, S.E., and Gouma, P.I., Ferroelectric WO(3) nanoparticles for acetone selective detection. Chemistry of Materials, 2008. 20(15): p. 4794-4796.

73. Yamazoe, N., New Approaches for Improving Semiconductor Gas Sensors. Sensors and Actuators B-Chemical, 1991. 5(1-4): p. 7-19.

74. Barsan, N., Schweizer-Berberich, M., and Gopel, W., Fundamental and practical aspects in the design of nanoscaled $\mathrm{SnO} 2$ gas sensors: a status report. Fresenius Journal of Analytical Chemistry, 1999. 365(4): p. 287-304.

75. Korotcenkov, G., Gas response control through structural and chemical modification of metal oxide films: state of the art and approaches. Sensors and Actuators B-Chemical, 2005. 107(1): p. 209-232.

76. Korotcenkov, G., Brinzari, V., Boris, Y., Ivanova, M., Schwank, J., and Morante, J., Influence of surface Pd doping on gas sensing characteristics of SnO2 thin films deposited by spray pirolysis. Thin Solid Films, 2003. 436(1): p. $119-126$.

77. Yamazoe, N., Kurokawa, Y., and Seiyama, T., Effects of Additives on Semiconductor Gas Sensors. Sensors and Actuators, 1983. 4(2): p. 283-289.

78. Barbi, G.B., Santos, J.P., Serrini, P., Gibson, P.N., Horrillo, M.C., and Manes, L., Ultrafine Grain-Size Tin-Oxide Films for Carbon-Monoxide Monitoring in Urban Environments. Sensors and Actuators B-Chemical, 1995. 25(1-3): p. 559-563.

79. Li, X.G., Ramasamy, R., and Dutta, P.K., Study of the resistance behavior of anatase and rutile thick films towards carbon monoxide and oxygen at high 
temperatures and possibilities for sensing applications. Sensors and Actuators B-Chemical, 2009. 143(1): p. 308-315.

80. Malagu, C., Fabbri, B., Gherardi, S., Giberti, A., Guidi, V., Landini, N., and Zonta, G., Chemoresistive Gas Sensors for the Detection of Colorectal Cancer Biomarkers. Sensors, 2014. 14(10): p. 18982-18992.

81. Iijima, S., Helical Microtubules of Graphitic Carbon. Nature, 1991. 354(6348): p. 56-58.

82. Kong, J., Franklin, N.R., Zhou, C.W., Chapline, M.G., Peng, S., Cho, K.J., and Dai, H.J., Nanotube molecular wires as chemical sensors. Science, 2000. 287(5453): p. 622-625.

83. Varghese, O.K., Kichambre, P.D., Gong, D., Ong, K.G., Dickey, E.C., and Grimes, C.A., Gas sensing characteristics of multi-wall carbon nanotubes. Sensors and Actuators B-Chemical, 2001. 81(1): p. 32-41.

84. Chung, J.Y., Lee, K.H., Lee, J., Troya, D., and Schatz, G.C., Multi-walled carbon nanotubes experiencing electrical breakdown as gas sensors. Nanotechnology, 2004. 15(11): p. 1596-1602.

85. Huang, C.S., Huang, B.R., Jang, Y.H., Tsai, M.S., and Yeh, C.Y., Threeterminal CNTs gas sensor for $\mathrm{N}-2$ detection. Diamond and Related Materials, 2005. 14(11-12): p. 1872-1875.

86. Zabet-Khosousi, A. and Dhirani, A.A., Charge transport in nanoparticle assemblies. Chemical Reviews, 2008. 108(10): p. 4072-4124.

87. Murray, R.W., Nanoelectrochemistry: Metal nanoparticles, nanoelectrodes, and nanopores. Chemical Reviews, 2008. 108(7): p. 2688-2720. 
88. Franke, M.E., Koplin, T.J., and Simon, U., Metal and metal oxide nanoparticles in chemiresistors: Does the nanoscale matter? (vol 2, pg 36, 2006). Small, 2006. 2(3): p. 301-301.

89. Haick, H., Chemical sensors based on molecularly modified metallic nanoparticles. Journal of Physics D-Applied Physics, 2007. 40(23): p. 71737186.

90. Daniel, M.C. and Astruc, D., Gold nanoparticles: Assembly, supramolecular chemistry, quantum-size-related properties, and applications toward biology, catalysis, and nanotechnology. Chemical Reviews, 2004. 104(1): p. 293-346.

91. Boisselier, E. and Astruc, D., Gold nanoparticles in nanomedicine: preparations, imaging, diagnostics, therapies and toxicity. Chemical Society Reviews, 2009. 38(6): p. 1759-1782.

92. Tisch, U. and Haick, H., Nanomaterials for cross-reactive sensor arrays. Mrs Bulletin, 2010. 35(10): p. 797-803.

93. Dovgolevsky, E., Konvalina, G., Tisch, U., and Haick, H., Mono layerCapped Cubic Platinum Nanoparticles for Sensing Nonpolar Analytes in Highly Humid Atmospheres. Journal of Physical Chemistry C, 2010. 114(33): p. $14042-14049$.

94. Wang, X.Y., Drew, C., Lee, S.H., Senecal, K.J., Kumar, J., and Sarnuelson, L.A., Electrospun nanofibrous membranes for highly sensitive optical sensors. Nano Letters, 2002. 2(11): p. 1273-1275. 
95. Kim, I.D., Rothschild, A., Lee, B.H., Kim, D.Y., Jo, S.M., and Tuller, H.L., Ultrasensitive chemiresistors based on electrospun TiO2 nanofibers. Nano Letters, 2006. 6(9): p. 2009-2013.

96. Hoth, C.N., Choulis, S.A., Schilinsky, P., and Brabec, C.J., High photovoltaic performance of inkjet printed polymer: Fullerene blends. Advanced Materials, 2007. 19(22): p. 3973-+.

97. Ji, S.L., Wang, X.J., Liu, C.F., Wang, H.B., Wang, T., and Yan, D.H., Controllable organic nanofiber network crystal room temperature NO2 sensor. Organic Electronics, 2013. 14(3): p. 821-826.

98. Choi, S.H., Choi, S.J., Min, B.K., Lee, W.Y., Park, J.S., and Kim, I.D., Facile Synthesis of p-type Perovskite SrTi0.65Fe0.3503 Nanofibers Prepared by Electrospinning and Their Oxygen-Sensing Properties. Macromolecular Materials and Engineering, 2013. 298(5): p. 521-527.

99. Zhang, Y.X., Kim, J.J., Chen, D., Tuller, H.L., and Rutledge, G.C., Electrospun Polyaniline Fibers as Highly Sensitive Room Temperature Chemiresistive Sensors for Ammonia and Nitrogen Dioxide Gases. Advanced Functional Materials, 2014. 24(25): p. 4005-4014.

100. Zhang, J., Liu, X.H., Wu, S.H., Xu, H.Y., and Cao, B.Q., One-pot fabrication of uniform polypyrrole/Au nanocomposites and investigation for gas sensing. Sensors and Actuators B-Chemical, 2013. 186: p. 695-700.

101. Stutzmann, N., Friend, R.H., and Sirringhaus, H., Self-aligned, verticalchannel, polymer field-effect transistors. Science, 2003. 299(5614): p. 18811884. 
102. Gelinck, G.H., Geuns, T.C.T., and de Leeuw, D.M., High-performance allpolymer integrated circuits. Applied Physics Letters, 2000. 77(10): p. 14871489.

103. Koezuka, H., Tsumura, A., and Ando, T., Field-Effect Transistor with Polythiophene Thin-Film. Synthetic Metals, 1987. 18(1-3): p. 699-704.

104. Bao, Z., Dodabalapur, A., and Lovinger, A.J., Soluble and processable regioregular poly(3-hexylthiophene) for thin film field-effect transistor applications with high mobility. Applied Physics Letters, 1996. 69(26): p. 4108-4110.

105. Pinto, N.J., Perez, R., Mueller, C.H., Theofylaktos, N., and Miranda, F.A., Dual input AND gate fabricated from a single channel poly(3-hexylthiophene) thin film field effect transistor. Journal of Applied Physics, 2006. 99(8).

106. Yu, J.H., Fridrikh, S.V., and Rutledge, G.C., The role of elasticity in the formation of electrospun fibers. Polymer, 2006. 47(13): p. 4789-4797.

107. Drury, C.J., Mutsaers, C.M.J., Hart, C.M., Matters, M., and de Leeuw, D.M., Low-cost all-polymer integrated circuits. Applied Physics Letters, 1998. 73(1): p. $108-110$.

108. Fridrikh, S.V., Yu, J.H., Brenner, M.P., and Rutledge, G.C., Controlling the fiber diameter during electrospinning. Physical Review Letters, 2003. 90(14).

109. Reneker, D.H. and Chun, I., Nanometre diameter fibres of polymer, produced by electrospinning. Nanotechnology, 1996. 7(3): p. 216-223.

110. Ding, B., Wang, M.R., Yu, J.Y., and Sun, G., Gas Sensors Based on Electrospun Nanofibers. Sensors, 2009. 9(3): p. 1609-1624. 
111. Queralto, N., Berliner, A.N., Goldsmith, B., Martino, R., Rhodes, P., and Lim, S.H., Detecting cancer by breath volatile organic compound analysis: a review of array-based sensors. Journal of Breath Research, 2014. 8(2).

112. Macagnano, A., Zampetti, E., Pantalei, S., De Cesare, F., Bearzotti, A., and Persaud, K.C., Nanofibrous PANI-based conductive polymers for trace gas analysis. Thin Solid Films, 2011. 520(3): p. 978-985.

113. Wohltjen, H. and Snow, A.W., Colloidal metal-insulator-metal ensemble chemiresistor sensor. Analytical Chemistry, 1998. 70(14): p. 2856-2859.

114. Evans, S.D., Johnson, S.R., Cheng, Y.L.L., and Shen, T.H., Vapour sensing using hybrid organic-inorganic nanostructured materials. Journal of Materials Chemistry, 2000. 10(1): p. 183-188.

115. Han, L., Daniel, D.R., Maye, M.M., and Zhong, C.J., Core-shell nanostructured nanoparticle films as chemically sensitive interfaces. Analytical Chemistry, 2001. 73(18): p. 4441-4449.

116. Zamborini, F.P., Leopold, M.C., Hicks, J.F., Kulesza, P.J., Malik, M.A., and Murray, R.W., Electron hopping conductivity and vapor sensing properties of flexible network polymer films of metal nanoparticles. Journal of the American Chemical Society, 2002. 124(30): p. 8958-8964.

117. Cai, Q.Y. and Zellers, E.T., Dual-chemiresistor GC detector employing layerprotected metal nanocluster interfaces. Analytical Chemistry, 2002. 74(14): p. 3533-3539. 
118. Grate, J.W., Nelson, D.A., and Skaggs, R., Sorptive behavior of monolayerprotected gold nanoparticle films: Implications for chemical vapor sensing. Analytical Chemistry, 2003. 75(8): p. 1868-1879.

119. Rowe, M.P., Plass, K.E., Kim, K., Kurdak, C., Zellers, E.T., and Matzger, A.J., Single-phase synthesis of functionalized gold nanoparticles. Chemistry of Materials, 2004. 16(18): p. 3513-3517.

120. Zhong, Q., Steinecker, W.H., and Zellers, E.T., Characterization of a highperformance portable GC with a chemiresistor array detector. Analyst, 2009. 134(2): p. 283-293.

121. Ibanez, F.J. and Zamborini, F.P., Chemiresistive sensing of volatile organic compounds with films of surfactant-stabilized gold and gold-silver alloy nanoparticles. Acs Nano, 2008. 2(8): p. 1543-1552.

122. Brust, M., Walker, M., Bethell, D., Schiffrin, D.J., and Whyman, R., Synthesis of Thiol-Derivatized Gold Nanoparticles in a 2-Phase Liquid-Liquid System. Journal of the Chemical Society-Chemical Communications, 1994(7): p. 801802.

123. Li, M.X., Biswas, S., Nantz, M.H., Higashi, R.M., and Fu, X.A., A microfabricated preconcentration device for breath analysis. Sensors and Actuators B-Chemical, 2013. 180: p. 130-136.

124. Brust, M., Fink, J., Bethell, D., Schiffrin, D.J., and Kiely, C., Synthesis and Reactions of Functionalized Gold Nanoparticles. Journal of the Chemical Society-Chemical Communications, 1995(16): p. 1655-1656. 
125. Nuzzo, R.G., Dubois, L.H., and Allara, D.L., Fundamental-Studies of Microscopic Wetting on Organic-Surfaces .1. Formation and Structural Characterization of a Self-Consistent Series of Polyfunctional Organic Monolayers. Journal of the American Chemical Society, 1990. 112(2): p. 558569.

126. Widrig, C.A., Chung, C., and Porter, M.D., The Electrochemical Desorption of N-Alkanethiol Monolayers from Polycrystalline Au and Ag Electrodes. Journal of Electroanalytical Chemistry, 1991. 310(1-2): p. 335-359.

127. Li, Y.Z., Huang, J.Y., Mciver, R.T., and Hemminger, J.C., Characterization of Thiol Self-Assembled Films by Laser Desorption Fourier-Transform MassSpectrometry. Journal of the American Chemical Society, 1992. 114(7): p. 2428-2432.

128. Konvalina, G. and Haick, H., Sensors for Breath Testing: From Nanomaterials to Comprehensive Disease Detection. Accounts of Chemical Research, 2014. 47(1): p. 66-76.

129. Luo, X.Z., Jia, X.J., Deng, J.H., Zhong, J.L., Liu, H.J., Wang, K.J., and Zhong, D.C., A Microporous Hydrogen-Bonded Organic Framework: Exceptional Stability and Highly Selective Adsorption of Gas and Liquid. Journal of the American Chemical Society, 2013. 135(32): p. 11684-11687.

130. Wang, H.L., Li, B., Wu, H., Hu, T.L., Yao, Z.Z., Zhou, W., Xiang, S.C., and Chen, B.L., A Flexible Microporous Hydrogen-Bonded Organic Framework for Gas Sorption and Separation. Journal of the American Chemical Society, 2015. 137(31): p. 9963-9970. 
131. Bregovic, V.B., Basaric, N., and Mlinaric-Majerski, K., Anion binding with urea and thiourea derivatives. Coordination Chemistry Reviews, 2015. 295: p. $80-124$.

132. Amendola, V., Fabbrizzi, L., and Mosca, L., Anion recognition by hydrogen bonding: urea-based receptors. Chemical Society Reviews, 2010. 39(10): p. 3889-3915.

133. Yabuuchi, K., Marfo-Owusu, E., and Kato, T., A new urea gelator: incorporation of intra- and intermolecular hydrogen bonding for stable $1 D$ self-assembly. Organic \& Biomolecular Chemistry, 2003. 1(19): p. 3464-3469.

134. Hooper, A.E., Kennedy, S.R., Jones, C.D., and Steed, J.W., Gelation by supramolecular dimerization of mono(urea)s. Chemical Communications, 2016. 52(1): p. 198-201.

135. Howlader, P., Das, P., Zangrando, E., and Mukherjee, P.S., UreaFunctionalized Self-Assembled Molecular Prism for Heterogeneous Catalysis in Water. Journal of the American Chemical Society, 2016. 138(5): p. 16681676.

136. Kaftory, M., Kapon, M., and Botoshanksy, M., Role of Hydrogen-Bonding in Determining the Crystal-Structures of the Adducts between Acetone and Urea Derivatives. Chemistry of Materials, 1994. 6(8): p. 1245-1249.

137. Tisch, U. and Haick, H., Arrays of chemisensitive monolayer-capped metallic nanoparticles for diagnostic breath testing. Reviews in Chemical Engineering, 2010. 26(5-6): p. 171-179. 
138. Chow, E., Muller, K.H., Davies, E., Raguse, B., Wieczorek, L., Cooper, J.S., and Hubble, L.J., Characterization of the Sensor Response of Gold Nanoparticle Chemiresistors. Journal of Physical Chemistry C, 2010. 114(41): p. $17529-17534$.

139. Zhang, H.L., Evans, S.D., Henderson, J.R., Miles, R.E., and Shen, T.H., Vapour sensing using surface functionalized gold nanoparticles. Nanotechnology, 2002. 13(3): p. 439-444.

140. Krasteva, N., Besnard, I., Guse, B., Bauer, R.E., Mullen, K., Yasuda, A., and Vossmeyer, T., Self-assembled gold nanoparticle/dendrimer composite films for vapor sensing applications. Nano Letters, 2002. 2(5): p. 551-555.

141. Ibanez, F.J., Gowrishetty, U., Crain, M.M., Walsh, K.M., and Zamborini, F.P., Chemiresistive vapor sensing with microscale films of gold monolayer protected clusters. Analytical Chemistry, 2006. 78(3): p. 753-761.

142. Joseph, Y., Guse, B., Vossmeyer, T., and Yasuda, A., Gold nanoparticle/organic networks as chemiresistor coatings: The effect of film morphology on vapor sensitivity. Journal of Physical Chemistry C, 2008. 112(32): p. 12507-12514.

143. Muller, K.H., Herrmann, J., Raguse, B., Baxter, G., and Reda, T., Percolation model for electron conduction in films of metal nanoparticles linked by organic molecules. Physical Review B, 2002. 66(7).

144. Konvalina, G. and Haick, H., Effect of Humidity on Nanoparticle-Based Chemiresistors: A Comparison between Synthetic and Real-World Samples. Acs Applied Materials \& Interfaces, 2012. 4(1): p. 317-325. 
145. Park, S. and Yousaf, M.N., An interfacial oxime reaction to immobilize ligands and cells in patterns and gradients to photoactive surfaces. Langmuir, 2008. 24(12): p. 6201-6207.

146. Willwacher, J., Rakshit, S., and Glorius, F., Investigating $N$-methoxy- $N$ '-aryl ureas in oxidative $\mathrm{C}-\mathrm{H}$ olefination reactions: an unexpected oxidation behaviour. Organic \& Biomolecular Chemistry, 2011. 9(13): p. 4736-4740.

147. Dendane, N., Hoang, A., Guillard, L., Defrancq, E., Vinet, F., and Dumy, P., Efficient surface patterning of oligonucleotides inside a glass capillary through oxime bond formation. Bioconjugate Chemistry, 2007. 18(3): p. 671676.

148. Parikh, K., Cattanach, K., Rao, R., Suh, D.S., Wu, A.M., and Manohar, S.K., Flexible vapour sensors using single walled carbon nanotubes. Sensors and Actuators B-Chemical, 2006. 113(1): p. 55-63.

149. Schoenfisch, M.H. and Pemberton, J.E., Air stability of alkanethiol selfassembled monolayers on silver and gold surfaces. Journal of the American Chemical Society, 1998. 120(18): p. 4502-4513.

150. Garg, N., Mohanty, A., Lazarus, N., Schultz, L., Rozzi, T.R., Santhanam, S., Weiss, L., Snyder, J.L., Fedder, G.K., and Jin, R.C., Robust gold nanoparticles stabilized by trithiol for application in chemiresistive sensors. Nanotechnology, 2010. 21(40).

151. Shi, Y.K. and Hu, F.B., The global implications of diabetes and cancer. Lancet, 2014. 383(9933): p. 1947-1948. 
152. Boland, E., Monsod, T., Delucia, M., Brandt, C.A., Fernando, S., and Tamborlane, W.V., Limitations of conventional methods of self-monitoring of blood glucose - Lessons learned from 3 days of continuous glucose sensing in pediatric patients with type 1 diabetes. Diabetes Care, 2001. 24(11): p. 18581862.

153. McGrath, L.T., Patrick, R., and Silke, B., Breath isoprene in patients with heart failure. European Journal of Heart Failure, 2001. 3(4): p. 423-427.

154. Risby, T.H. and Sehnert, S.S., Clinical application of breath biomarkers of oxidative stress status. Free Radical Biology and Medicine, 1999. 27(11-12): p. 1182-1192.

155. Kneepkens, C.M.F., Lepage, G., and Roy, C.C., The Potential of the Hydrocarbon Breath Test as a Measure of Lipid-Peroxidation. Free Radical Biology and Medicine, 1994. 17(2): p. 127-160.

156. Kupari, M., Lommi, J., Ventila, M., and Karjalainen, U., Break Acetone in Congestive-Heart-Failure. American Journal of Cardiology, 1995. 76(14): p. 1076-\&.

157. Cao, W.Q. and Duan, Y.X., Breath analysis: Potential for clinical diagnosis and exposure assessment. Clinical Chemistry, 2006. 52(5): p. 800-811.

158. Ping, W., Yi, T., Xie, H.B., and Shen, F.R., A novel method for diabetes diagnosis based on electronic nose. Biosensors \& Bioelectronics, 1997. 12(910): p. 1031-1036.

159. Turner, C., Spanel, P., and Smith, D., A longitudinal study of ammonia, acetone and propanol in the exhaled breath of 30 subjects using selected ion 
flow tube mass spectrometry, SIFT-MS. Physiological Measurement, 2006. 27(4): p. 321-337.

160. Sulway, M.J. and Malins, J.M., Acetone in Diabetic Ketoacidosis. Lancet, 1970. 2(7676): p. 736-\&.

161. Kalapos, M.P., On the mammalian acetone metabolism: from chemistry to clinical implications. Biochimica Et Biophysica Acta-General Subjects, 2003. 1621(2): p. 122-139.

162. Li, M.X., A microreactor approach for chemoselective capture and analysis of carbonyl compounds in air and exhaled breath. University of Louisville, 2013

163. Pang, P.F., Guo, Z.D., and Cai, Q.Y., Humidity effect on the monolayerprotected gold nanoparticles coated chemiresistor sensor for VOCs analysis. Talanta, 2005. 65(5): p. 1343-1348.

164. Yu, J.B., Byun, H.G., So, M.S., and Huh, J.S., Analysis of diabetic patient's breath with conducting polymer sensor array. Sensors and Actuators BChemical, 2005. 108(1-2): p. 305-308.

165. Cooper, J.S., Raguse, B., Chow, E., Hubble, L., Muller, K.H., and Wieczorek, L., Gold Nanoparticle Chemiresistor Sensor Array that Differentiates between Hydrocarbon Fuels Dissolved in Artificial Seawater. Analytical Chemistry, 2010. 82(9): p. 3788-3795.

166. Neri, G., Bonavita, A., Rizzo, G., Galvagno, S., Donato, N., and Caputi, L.S., A study of water influence on CO response on gold-doped iron oxide sensors. Sensors and Actuators B-Chemical, 2004. 101(1-2): p. 90-96. 
167. Chai, M. and Pawliszyn, J., Analysis of Environmental Air Samples by SolidPhase Microextraction and Gas-Chromatography Ion-Trap MassSpectrometry. Environmental Science \& Technology, 1995. 29(3): p. 693-701.

168. Hyspler, R., Crhova, S., Gasparic, J., Zadak, Z., Cizkova, M., and Balasova, V., Determination of isoprene in human expired breath using solid-phase microextraction and gas chromatography-mass spectrometry. Journal of Chromatography B, 2000. 739(1): p. 183-190.

169. Hosseini, M.W., Blacker, A.J., and Lehn, J.M., Multiple Molecular Recognition and Catalysis - a Multifunctional Anion Receptor Bearing an Anion Binding-Site, an Intercalating Group, and a Catalytic Site for Nucleotide Binding and Hydrolysis. Journal of the American Chemical Society, 1990. 112(10): p. 3896-3904.

170. Lin, S. and Jacobsen, E.N., Thiourea-catalysed ring opening of episulfonium ions with indole derivatives by means of stabilizing non-covalent interactions. Nature Chemistry, 2012. 4(10): p. 817-824.

171. Guinn, E.J., Schwinefus, J.J., Cha, H.K., McDevitt, J.L., Merker, W.E., Ritzer, R., Muth, G.W., Engelsgjerd, S.W., Mangold, K.E., Thompson, P.J., Kerins, M.J., and Record, M.T., Quantifying Functional Group Interactions That Determine Urea Effects on Nucleic Acid Helix Formation (vol 135, pg 5828, 2013). Journal of the American Chemical Society, 2013. 135(24): p. 92209220.

172. Grekov, A.P., Veselov, V. Y., The $\alpha$-Effect in the Chemistry of Organic Compounds Chemical Reviews, 1978. 47: p. 631-648. 
173. Edwards, J.O., Pearson, R. G., The factors determining nucleophilic reactivities. J. Am. Chem. Soc., 1962. 84: p. 16-21.

174. Bordwell, F.G., Fried, H.E., Hughes, D.L., Lynch, T.Y., Satish, A.V., and Whang, Y.E., Acidities of Carboxamides, Hydroxamic Acids, Carbohydrazides, Benzenesulfonamides, and Benzenesulfonohydrazides in Dmso Solution. Journal of Organic Chemistry, 1990. 55(10): p. 3330-3336.

175. Bordwell, F.G., Algrim, D.J., and Harrelson, J.A., The Relative Ease of Removing a Proton, a Hydrogen-Atom, or an Electron from Carboxamides Versus Thiocarboxamides. Journal of the American Chemical Society, 1988. 110(17): p. 5903-5904.

176. Bordwell, F.G. and Ji, G.Z., Effects of Structural-Changes on Acidities and Homolytic Bond-Dissociation Energies of the $H-N$ Bonds in Amidines, Carboxamides, and Thiocarboxamides. Journal of the American Chemical Society, 1991. 113(22): p. 8398-8401.

177. Bordwell, F.G., Equilibrium Acidities in Dimethyl-Sulfoxide Solution. Accounts of Chemical Research, 1988. 21(12): p. 456-463. 


\title{
CURRICULUM VITAE
}

\author{
Zhenzhen Xie \\ Email: zhenzhen.xie@louisville.edu
}

\section{Education}

Ph.D in Chemical Engineering

University of Louisville, Louisville, KY, US

Master of Science in Chemical Engineering

University of Louisville, Louisville, KY, US

Bachelor of Science in Material Chemistry

Heilongjiang University, Harbin, Heilongjiang, China
May 2017

GPA: 3.8

August 2013

GPA: 3.8

May 2011

GPA: 3.7

\section{Relevant Experience}

Chemiresistive sensing of volatile organic compounds (VOCs) with films of nanomaterials - nanoparticles and nanofibers

- Developed a non-invasive method for VOC analysis with detection limit as low as $0.1 \mathrm{ppb}$.

- Designed and fabricated interdigitated electrode in clean room using CAD and photolithography.

- Improved sensor's sensitivity and selectivity for acetone detection by 12 times higher using urea thiol functionalized gold nanoparticles. 
- Fabricated different sizes of polymer nanofibers using electrospinning by changing viscosity, injection rate, distance and high voltage.

- Enhanced the performance of the sensors from understanding the chemical and physical characteristics of nanoparticles and polymers via XRD, SEM, TEM, Raman, FT-IR, TGA and size distribution etc.

\section{MOF-based catalytic performance and membrane for gas separation}

- Prepared various zeolites and the crystal size is $92 \%$ smaller using microwave heating than hydrothermal treatment.

- Enhanced the catalytic performance of SAPO-56 by $42.6 \%$ using microwaveassisted synthesis for conversion of $\mathrm{CO}_{2}$ to carbonates.

- Fabricated membranes on different supports for the separation of $\mathrm{CO}_{2}$ from natural gas, which can considerably reduce the cost of natural gas purification.

- Improved $\mathrm{CO}_{2} / \mathrm{CH}_{4}$ selectivity by three-fold through synthesized Bio-MOF 13 and Bio-MOF 14 membranes compared to that of original Bio-MOF membrane.

\section{Teaching/mentoring}

- Assisted in teaching graduate courses, including Transport Phenomena and Chemical Engineering Analysis.

- Trained students to operate gas sensor equipment, reactor, BET, SEM, XRD etc.

- Mentored undergraduate and high school students on catalysis and gas sensor projects.

\section{Selected publications}


- Z. Xie, S. Mandapati*, A. Steward, M. Nantz, X. Fu, "Actuating gold nanoparticle chemiresistor sensitivity and selectivity by incorporation of a molecular recognition motif", Sensors and Actuators, (under review).

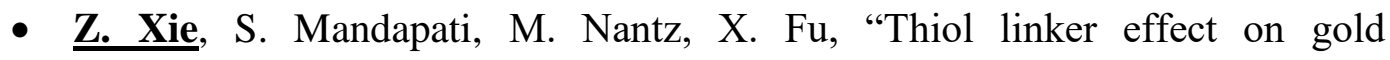
nanoparticle chemiresistor sensor for the detection of acetone", (in preparation).

- $\underline{\text { Z. Xie, }}$, T. Li, N.L. Rosi, M.A. Carreon, "Alumina-supported cobalt-adeninate MOF membranes for $\mathrm{CO}_{2} / \mathrm{CH}_{4}$ separation", Journal of Material Chemistry $\mathrm{A}$ 2014, 2, 1239.

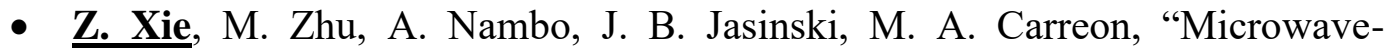
assisted synthesized SAPO-56 as a catalyst in the conversion of $\mathrm{CO}_{2}$ to cyclic carbonates", Dalton Transactions 2013, 42, 6732

\section{Selected presentations}

- Detection of acetone in exhaled breath by gold nanoparticle gas sensors," (2015), AIChE Annual Meeting, Micro and Nanofabricated Sensors, Salt Lake City, UT.

- "Microwave-assisted synthesized SAPO-56 as catalyst in the conversion of $\mathrm{CO}_{2}$ to cyclic carbonates," (2013), $23^{\text {rd }}$ North American Catalysis Society Meeting, Energy \& Sustainability I, Louisville, KY.

- "Microwave-assisted synthesized SAPO-56 as a catalyst in the conversion of $\mathrm{CO}_{2}$ to cyclic carbonates," (2012), AIChE Annual Meeting, Catalysis and Reaction Engineering Division, Pittsburgh, PA. 


\section{Key skills}

- Characterization: XRD, SEM, TEM, BET, FT-IR, Raman, TGA

- Chromatography: GC-MS, HPLC

- Software: Labview, Origin, ANOVA, Microsoft Office, Chemdraw

\section{Honors, Awards and Membership}

- Graduate Dean's Citation Award, University of Louisville, 2017

- University Scholarship and Tuition Award, University of Louisville, 2016

- Academic Excellence Scholarship, Heilongjiang University, China, 20072011

- American Institute of Chemical Engineers.

- Vice President/Treasure of Chinese Student and Scholar Association. 